\title{
Two-Dimensional Gas Chromatographic and Mass Spectrometric Characterization of Lipid-rich Biological Matrices-Application to Human Cerumen (Earwax)
}

\author{
Allix M. Coon ${ }^{1}$, A. John Dane ${ }^{2}$, Gavin Setzen ${ }^{3}$, Robert B. Cody ${ }^{2}$ and Rabi A. Musah ${ }^{1 *}$ \\ ${ }^{1}$ Department of Chemistry, State University of New York at Albany, 1400 Washington Ave, \\ Albany, NY 12222 \\ ${ }^{2}$ JEOL USA Inc., 11 Dearborn Rd, Peabody, MA 01960 \\ ${ }^{3}$ Albany ENT and Allergy Services, 123 Everett Rd, Albany, NY 12205 \\ *Corresponding author
}

\section{SUPPLEMENTARY DATA}

Tables S1 though S39 show the high-resolution mass data $(\mathrm{m} / \mathrm{z}$ values and the corresponding ion counts) for each of the indicated compounds that was identified in earwax. 
Table S1. High-resolution mass table for the compound identified as dodecene.

\begin{tabular}{|c|c|c|c|c|c|c|c|c|c|}
\hline Mass & $\begin{array}{c}\text { Ion } \\
\text { counts }\end{array}$ & Mass & $\begin{array}{c}\text { Ion } \\
\text { counts }\end{array}$ & Mass & $\begin{array}{c}\text { Ion } \\
\text { counts }\end{array}$ & Mass & $\begin{array}{c}\text { Ion } \\
\text { counts }\end{array}$ & Mass & $\begin{array}{c}\text { Ion } \\
\text { counts }\end{array}$ \\
\hline 50.019 & 1809 & 56.588 & 2743 & 68.066 & 48385 & 74.042 & 814 & 97.544 & 641 \\
\hline 51.027 & 5310 & 56.848 & 2175 & 68.135 & 1311 & 77.043 & 4302 & 97.816 & 1118 \\
\hline 52.035 & 3417 & 57.038 & 4951 & 68.634 & 353 & 78.050 & 1792 & 97.863 & 406 \\
\hline 53.007 & 335 & 57.074 & 234443 & 68.691 & 411 & 79.059 & 6348 & 97.893 & 410 \\
\hline 53.043 & 35956 & 57.102 & 630 & 68.936 & 418 & 80.065 & 1678 & 98.061 & 519 \\
\hline 53.106 & 606 & 57.115 & 2523 & 68.968 & 440 & 81.075 & 18077 & 98.113 & 60947 \\
\hline 53.310 & 281 & 57.318 & 2172 & 69.054 & 422 & 82.082 & 46353 & 98.472 & 896 \\
\hline 54.011 & 351 & 57.347 & 1756 & 69.074 & 288698 & 82.444 & 421 & 98.517 & 747 \\
\hline 54.051 & 57480 & 57.374 & 2790 & 69.103 & 278 & 83.090 & 211282 & 98.819 & 592 \\
\hline 54.315 & 584 & 57.586 & 2111 & 69.347 & 1575 & 83.139 & 1812 & 98.862 & 345 \\
\hline 54.342 & 519 & 57.618 & 2260 & 69.375 & 1581 & 83.392 & 1121 & 99.049 & 373 \\
\hline 54.373 & 416 & 57.847 & 1750 & 69.408 & 2488 & 83.420 & 1061 & 99.077 & 385 \\
\hline 54.573 & 464 & 57.881 & 1467 & 69.485 & 859 & 83.453 & 1825 & 99.118 & 5686 \\
\hline 54.623 & 397 & 58.010 & 935 & 69.631 & 1656 & 83.485 & 1380 & 99.446 & 414 \\
\hline 54.795 & 423 & 58.045 & 1390 & 69.661 & 2110 & 83.546 & 295 & 99.767 & 387 \\
\hline 54.825 & 444 & 58.078 & 10621 & 69.933 & 1653 & 83.677 & 423 & 105.074 & 270 \\
\hline 54.900 & 329 & 58.117 & 1455 & 69.967 & 1563 & 83.738 & 1144 & 107.089 & 276 \\
\hline 54.980 & 547 & 58.337 & 899 & 70.045 & 997 & 83.766 & 1359 & 109.107 & 1002 \\
\hline 55.022 & 3072 & 58.361 & 478 & 70.082 & 267258 & 84.098 & 140844 & 110.114 & 5833 \\
\hline 55.059 & 388706 & 58.384 & 603 & 70.358 & 893 & 84.351 & 450 & 111.122 & 40771 \\
\hline 55.083 & 344 & 58.581 & 488 & 70.385 & 1371 & 84.398 & 974 & 111.509 & 361 \\
\hline 55.098 & 2838 & 58.607 & 590 & 70.423 & 2374 & 84.429 & 1011 & 112.129 & 19313 \\
\hline 55.303 & 2390 & 58.801 & 329 & 70.474 & 842 & 84.481 & 789 & 112.214 & 700 \\
\hline 55.327 & 2261 & 58.837 & 464 & 70.497 & 831 & 84.700 & 477 & 113.133 & 1899 \\
\hline 55.358 & 3465 & 58.877 & 421 & 70.675 & 1566 & 84.754 & 526 & 124.130 & 2180 \\
\hline 55.384 & 2414 & 59.080 & 494 & 70.717 & 1754 & 85.017 & 446 & 125.137 & 13545 \\
\hline 55.407 & 660 & 60.025 & 6257 & 70.942 & 1035 & 85.105 & 41134 & 125.244 & 461 \\
\hline 55.425 & 767 & 61.033 & 55696 & 70.966 & 1035 & 85.178 & 714 & 126.145 & 11023 \\
\hline 55.576 & 2957 & 61.315 & 274 & 70.997 & 1097 & 86.109 & 2451 & 126.223 & 441 \\
\hline 55.606 & 2890 & 61.369 & 381 & 71.052 & 487 & 89.041 & 287 & 127.149 & 1437 \\
\hline 55.831 & 2334 & 61.613 & 317 & 71.090 & 86181 & 91.059 & 1592 & 138.145 & 919 \\
\hline 55.996 & 693 & 62.038 & 2630 & 71.402 & 377 & 92.064 & 257 & 139.153 & 3725 \\
\hline 56.030 & 3435 & 63.028 & 1082 & 71.432 & 399 & 93.074 & 842 & 140.161 & 11086 \\
\hline 56.066 & 324023 & 64.034 & 291 & 71.465 & 603 & 94.081 & 282 & 141.164 & 1379 \\
\hline 56.106 & 3319 & 65.043 & 6279 & 71.508 & 537 & 95.090 & 4693 & 166.177 & 309 \\
\hline 56.272 & 772 & 66.051 & 4807 & 71.760 & 438 & 96.098 & 16927 & 168.193 & 7902 \\
\hline 56.311 & 2987 & 67.059 & 56016 & 71.789 & 354 & 97.070 & 400 & 169.196 & 1392 \\
\hline 56.337 & 2216 & 67.350 & 316 & 72.025 & 632 & 97.106 & 143517 & 234.945 & 313 \\
\hline 56.366 & 3570 & 67.425 & 368 & 72.093 & 4183 & 97.462 & 1174 & 249.943 & 422 \\
\hline 56.436 & 406 & 67.706 & 343 & 73.032 & 630 & 97.498 & 1293 & 251.949 & 574 \\
\hline
\end{tabular}


Table S2. High-resolution mass table for the compound identified as dodecane.

\begin{tabular}{|c|c|c|c|c|c|c|c|}
\hline Mass & $\begin{array}{c}\text { Ion } \\
\text { counts }\end{array}$ & Mass & $\begin{array}{c}\text { Ion } \\
\text { counts }\end{array}$ & Mass & $\begin{array}{c}\text { Ion } \\
\text { counts }\end{array}$ & Mass & $\begin{array}{c}\text { Ion } \\
\text { counts }\end{array}$ \\
\hline 51.025 & 254 & 57.349 & 225 & 70.082 & 6596 & 97.107 & 861 \\
\hline 52.035 & 310 & 57.372 & 435 & 71.090 & 28078 & 98.114 & 2516 \\
\hline 53.043 & 1244 & 57.400 & 372 & 72.093 & 1759 & 99.122 & 2300 \\
\hline 54.050 & 1089 & 57.614 & 383 & 73.031 & 321 & 111.119 & 310 \\
\hline 55.022 & 669 & 58.045 & 361 & 77.041 & 337 & 112.129 & 1395 \\
\hline 55.059 & 10067 & 58.078 & 2757 & 78.051 & 344 & 113.138 & 1354 \\
\hline 56.030 & 657 & 60.025 & 1026 & 81.073 & 271 & 126.146 & 659 \\
\hline 56.066 & 9487 & 61.033 & 3837 & 82.082 & 546 & 127.153 & 1174 \\
\hline 57.038 & 886 & 67.059 & 1058 & 83.090 & 1912 & 140.161 & 259 \\
\hline 57.074 & 54355 & 68.066 & 661 & 84.098 & 3725 & 141.168 & 633 \\
\hline 57.134 & 1426 & 69.074 & 3856 & 85.106 & 15633 & 170.209 & 1011 \\
\hline 57.165 & 592 & 70.046 & 606 & 86.109 & 1221 & 251.951 & 340 \\
\hline
\end{tabular}


Table S3. High-resolution mass table for the compound identified as tetradecene.

\begin{tabular}{|c|c|c|c|c|c|c|c|}
\hline Mass & $\begin{array}{c}\text { Ion } \\
\text { counts }\end{array}$ & Mass & $\begin{array}{c}\text { Ion } \\
\text { counts }\end{array}$ & Mass & $\begin{array}{c}\text { Ion } \\
\text { counts }\end{array}$ & Mass & $\begin{array}{c}\text { Ion } \\
\text { counts }\end{array}$ \\
\hline 49.011 & 258 & 57.381 & 8268 & 67.990 & 389 & 71.961 & 621 \\
\hline 50.019 & 2675 & 57.430 & 2055 & 68.066 & 135738 & 71.986 & 446 \\
\hline 51.027 & 7913 & 57.446 & 742 & 68.342 & 497 & 72.016 & 1060 \\
\hline 52.035 & 5491 & 57.454 & 248 & 68.365 & 586 & 72.093 & 15058 \\
\hline 53.043 & 73850 & 57.620 & 6765 & 68.390 & 887 & 72.278 & 691 \\
\hline 53.306 & 517 & 57.855 & 4975 & 68.421 & 1391 & 72.308 & 528 \\
\hline 53.327 & 631 & 58.045 & 1860 & 68.478 & 397 & 72.526 & 443 \\
\hline 53.359 & 452 & 58.077 & 35210 & 68.651 & 1346 & 72.578 & 417 \\
\hline 53.588 & 410 & 58.132 & 3915 & 68.695 & 1282 & 73.035 & 389 \\
\hline 53.991 & 402 & 58.331 & 2763 & 68.725 & 896 & 73.095 & 610 \\
\hline 54.050 & 141675 & 58.359 & 2660 & 68.933 & 1457 & 76.037 & 337 \\
\hline 54.292 & 1029 & 58.379 & 1047 & 68.981 & 1042 & 77.043 & 9387 \\
\hline 54.317 & 1040 & 58.390 & 1952 & 69.004 & 893 & 78.050 & 2990 \\
\hline 54.343 & 1432 & 58.604 & 2137 & 69.042 & 1458 & 79.059 & 16907 \\
\hline 54.553 & 837 & 58.633 & 1674 & 69.074 & 640468 & 79.123 & 473 \\
\hline 54.582 & 934 & 58.798 & 722 & 69.316 & 2702 & 80.066 & 4297 \\
\hline 54.625 & 470 & 58.830 & 1320 & 69.357 & 3069 & 81.074 & 58758 \\
\hline 54.789 & 916 & 58.853 & 1586 & 69.375 & 4150 & 81.367 & 454 \\
\hline 54.830 & 1070 & 59.025 & 346 & 69.412 & 6899 & 81.399 & 353 \\
\hline 54.858 & 939 & 59.054 & 783 & 69.462 & 2277 & 81.419 & 326 \\
\hline 54.896 & 585 & 59.081 & 1595 & 69.483 & 2027 & 81.456 & 680 \\
\hline 54.924 & 450 & 59.109 & 944 & 69.668 & 5669 & 81.498 & 387 \\
\hline 54.973 & 897 & 59.139 & 712 & 69.702 & 5116 & 81.707 & 537 \\
\hline 55.026 & 2365 & 59.286 & 354 & 69.886 & 1749 & 82.082 & 154539 \\
\hline 55.059 & 757107 & 59.323 & 639 & 69.916 & 3856 & 82.326 & 424 \\
\hline 55.098 & 58297 & 59.352 & 530 & 69.950 & 4027 & 82.377 & 1090 \\
\hline 55.327 & 7063 & 59.388 & 526 & 69.993 & 2761 & 82.410 & 1144 \\
\hline 55.362 & 8892 & 59.581 & 487 & 70.047 & 1607 & 82.449 & 1583 \\
\hline 55.407 & 4199 & 59.617 & 523 & 70.082 & 592049 & 82.483 & 1224 \\
\hline 55.425 & 1965 & 59.835 & 493 & 70.125 & 14858 & 82.534 & 473 \\
\hline 55.593 & 7280 & 59.891 & 397 & 70.167 & 14873 & 82.636 & 473 \\
\hline 55.794 & 4032 & 60.025 & 1467 & 70.217 & 6764 & 82.678 & 686 \\
\hline 55.824 & 5525 & 60.121 & 384 & 70.268 & 943 & 82.707 & 1069 \\
\hline 56.035 & 3877 & 61.033 & 2301 & 70.364 & 2377 & 82.751 & 1579 \\
\hline 56.066 & 603466 & 63.028 & 1313 & 70.385 & 3036 & 82.863 & 412 \\
\hline 56.104 & 23450 & 64.032 & 249 & 70.421 & 5744 & 82.978 & 1648 \\
\hline 56.285 & 3470 & 65.043 & 13203 & 70.455 & 4412 & 83.014 & 1801 \\
\hline 56.318 & 5949 & 66.050 & 11282 & 70.475 & 1701 & 83.054 & 2794 \\
\hline 56.337 & 4915 & 67.058 & 153298 & 70.493 & 2475 & 83.090 & 619538 \\
\hline 56.367 & 7615 & 67.103 & 1321 & 70.695 & 4569 & 83.136 & 20353 \\
\hline 56.418 & 1189 & 67.332 & 915 & 70.728 & 4067 & 83.356 & 2615 \\
\hline 56.437 & 855 & 67.355 & 926 & 70.975 & 3197 & 83.396 & 4112 \\
\hline 56.575 & 5603 & 67.382 & 1348 & 71.089 & 293924 & 83.420 & 4292 \\
\hline 56.605 & 6110 & 67.418 & 810 & 71.135 & 2149 & 83.454 & 6890 \\
\hline 56.825 & 4838 & 67.443 & 497 & 71.367 & 762 & 83.518 & 1352 \\
\hline 56.855 & 4581 & 67.637 & 1361 & 71.395 & 1051 & 83.538 & 983 \\
\hline 57.040 & 3881 & 67.667 & 1093 & 71.476 & 2267 & 83.548 & 948 \\
\hline 57.074 & 648400 & 67.694 & 861 & 71.510 & 1756 & 83.717 & 4889 \\
\hline 57.113 & 37110 & 67.870 & 812 & 71.568 & 312 & 83.757 & 5200 \\
\hline 57.295 & 4948 & 67.897 & 744 & 71.692 & 1668 & 84.001 & 3180 \\
\hline 57.331 & 4901 & 67.925 & 797 & 71.725 & 1688 & 84.033 & 3993 \\
\hline 57.347 & 5882 & 67.956 & 629 & 71.925 & 351 & 84.064 & 2921 \\
\hline
\end{tabular}


Table S3 (continued). High-resolution mass table for the compound identified as tetradecene.

\begin{tabular}{|c|c|c|c|c|c|c|c|}
\hline Mass & $\begin{array}{c}\text { Ion } \\
\text { counts }\end{array}$ & Mass & $\begin{array}{c}\text { Ion } \\
\text { counts }\end{array}$ & Mass & $\begin{array}{c}\text { Ion } \\
\text { counts }\end{array}$ & Mass & $\begin{array}{c}\text { Ion } \\
\text { counts }\end{array}$ \\
\hline 84.097 & 359578 & 96.866 & 533 & 107.091 & 432 & 121.106 & 336 \\
\hline 84.346 & 2182 & 96.915 & 435 & 108.097 & 409 & 123.121 & 1670 \\
\hline 84.395 & 3241 & 97.078 & 1480 & 109.106 & 5075 & 124.130 & 9966 \\
\hline 84.429 & 1971 & 97.106 & 478808 & 110.114 & 24190 & 124.214 & 391 \\
\hline 84.457 & 2667 & 97.430 & 3164 & 110.175 & 322 & 125.137 & 55017 \\
\hline 84.555 & 405 & 97.462 & 2717 & 110.205 & 455 & 125.230 & 1758 \\
\hline 84.625 & 509 & 97.493 & 4000 & 111.097 & 601 & 125.568 & 491 \\
\hline 84.683 & 1540 & 97.530 & 3953 & 111.122 & 174146 & 126.145 & 44120 \\
\hline 84.716 & 1896 & 97.593 & 811 & 111.474 & 835 & 126.590 & 466 \\
\hline 85.032 & 2223 & 97.821 & 3927 & 111.502 & 996 & 127.149 & 4570 \\
\hline 85.070 & 1222 & 97.882 & 1815 & 111.539 & 1613 & 137.137 & 581 \\
\hline 85.105 & 133369 & 98.113 & 168759 & 111.888 & 1394 & 138.146 & 4176 \\
\hline 85.402 & 669 & 98.437 & 2856 & 111.942 & 564 & 139.153 & 10672 \\
\hline 85.440 & 707 & 98.470 & 2129 & 112.129 & 82997 & 140.161 & 15266 \\
\hline 85.479 & 711 & 98.506 & 2999 & 112.214 & 3562 & 140.228 & 415 \\
\hline 85.713 & 435 & 98.784 & 2378 & 112.259 & 1988 & 141.165 & 1984 \\
\hline 86.109 & 8002 & 98.824 & 2282 & 112.484 & 599 & 152.161 & 1983 \\
\hline 87.111 & 242 & 99.119 & 18613 & 112.522 & 1072 & 153.169 & 4804 \\
\hline 91.059 & 3644 & 99.165 & 1532 & 112.551 & 1094 & 154.177 & 9409 \\
\hline 92.065 & 670 & 99.429 & 1080 & 112.591 & 1243 & 155.180 & 1359 \\
\hline 93.075 & 2211 & 99.463 & 1334 & 112.885 & 1012 & 166.176 & 835 \\
\hline 94.083 & 1295 & 99.762 & 993 & 112.924 & 968 & 167.185 & 2506 \\
\hline 95.090 & 18865 & 99.833 & 516 & 113.134 & 8509 & 168.192 & 15717 \\
\hline 95.165 & 607 & 100.052 & 452 & 113.213 & 831 & 168.265 & 497 \\
\hline 95.206 & 435 & 100.124 & 1553 & 113.255 & 673 & 169.197 & 2345 \\
\hline 96.098 & 57600 & 100.177 & 383 & 113.299 & 363 & 194.208 & 431 \\
\hline 96.451 & 690 & 100.420 & 465 & 113.518 & 326 & 196.224 & 11343 \\
\hline 96.485 & 392 & 100.468 & 445 & 113.596 & 496 & 197.226 & 1834 \\
\hline 96.745 & 438 & 100.725 & 410 & 113.952 & 549 & 234.947 & 252 \\
\hline 96.783 & 449 & 105.074 & 698 & 114.136 & 398 & 249.948 & 449 \\
\hline 96.820 & 487 & 106.079 & 372 & 117.074 & 435 & 251.948 & 920 \\
\hline
\end{tabular}


Table S4. High-resolution mass table for the compound identified as tetradecane.

\begin{tabular}{|c|c|c|c|c|c|c|c|c|c|}
\hline Mass & $\begin{array}{c}\text { Ion } \\
\text { counts }\end{array}$ & Mass & $\begin{array}{c}\text { Ion } \\
\text { counts }\end{array}$ & Mass & $\begin{array}{c}\text { Ion } \\
\text { counts }\end{array}$ & Mass & $\begin{array}{c}\text { Ion } \\
\text { counts }\end{array}$ & Mass & $\begin{array}{c}\text { Ion } \\
\text { counts }\end{array}$ \\
\hline 50.018 & 281 & 57.848 & 1623 & 71.059 & 503 & 82.082 & 3236 & 99.203 & 551 \\
\hline 51.027 & 675 & 57.880 & 1573 & 71.090 & 168648 & 83.090 & 12247 & 100.124 & 1478 \\
\hline 52.035 & 428 & 58.047 & 608 & 71.370 & 815 & 84.098 & 16887 & 110.113 & 375 \\
\hline 53.043 & 5753 & 58.077 & 12917 & 71.394 & 868 & 84.169 & 417 & 111.122 & 2574 \\
\hline 54.050 & 5754 & 58.133 & 1340 & 71.418 & 1438 & 85.070 & 315 & 112.129 & 6632 \\
\hline 55.023 & 459 & 58.348 & 754 & 71.454 & 1299 & 85.105 & 102921 & 113.137 & 7901 \\
\hline 55.058 & 53355 & 58.387 & 703 & 71.510 & 415 & 85.152 & 894 & 114.140 & 863 \\
\hline 55.119 & 1435 & 58.583 & 486 & 71.645 & 478 & 85.412 & 468 & 125.137 & 760 \\
\hline 55.326 & 289 & 58.631 & 455 & 71.680 & 913 & 85.440 & 622 & 126.145 & 4122 \\
\hline 56.029 & 447 & 58.840 & 306 & 71.706 & 981 & 85.474 & 825 & 127.153 & 5275 \\
\hline 56.066 & 43514 & 58.866 & 445 & 71.936 & 478 & 85.519 & 504 & 128.158 & 480 \\
\hline 56.342 & 413 & 59.081 & 499 & 71.957 & 334 & 85.712 & 444 & 140.161 & 2628 \\
\hline 56.381 & 361 & 60.027 & 1272 & 71.984 & 614 & 85.768 & 653 & 141.168 & 3727 \\
\hline 56.605 & 413 & 61.033 & 1840 & 72.093 & 8777 & 86.037 & 479 & 142.172 & 403 \\
\hline 56.860 & 401 & 65.043 & 1066 & 72.255 & 482 & 86.109 & 6849 & 154.177 & 1212 \\
\hline 57.038 & 937 & 66.051 & 469 & 73.034 & 374 & 86.383 & 418 & 155.184 & 2349 \\
\hline 57.074 & 296033 & 67.058 & 4996 & 73.100 & 376 & 91.059 & 421 & 168.194 & 675 \\
\hline 57.321 & 1824 & 68.066 & 4038 & 77.042 & 1046 & 95.089 & 527 & 169.200 & 1355 \\
\hline 57.347 & 1770 & 69.074 & 23786 & 78.050 & 640 & 96.098 & 1293 & 196.223 & 333 \\
\hline 57.373 & 2703 & 69.138 & 486 & 79.059 & 1289 & 97.106 & 7827 & 198.239 & 2784 \\
\hline 57.446 & 622 & 70.044 & 701 & 80.066 & 320 & 98.113 & 10787 & 199.244 & 391 \\
\hline 57.612 & 2235 & 70.082 & 32136 & 81.074 & 1615 & 99.121 & 16954 & 251.943 & 417 \\
\hline
\end{tabular}


Table S5. High-resolution mass table for the compound identified as hexadecene.

\begin{tabular}{|c|c|c|c|c|c|c|c|}
\hline Mass & $\begin{array}{c}\text { Ion } \\
\text { counts }\end{array}$ & Mass & $\begin{array}{c}\text { Ion } \\
\text { counts }\end{array}$ & Mass & $\begin{array}{c}\text { Ion } \\
\text { counts }\end{array}$ & Mass & $\begin{array}{c}\text { Ion } \\
\text { counts }\end{array}$ \\
\hline 53.042 & 30885 & 57.837 & 2247 & 70.384 & 1438 & 84.097 & 152112 \\
\hline 53.106 & 665 & 57.868 & 2281 & 70.413 & 2158 & 84.343 & 805 \\
\hline 53.318 & 392 & 58.047 & 1136 & 70.440 & 2612 & 84.387 & 1108 \\
\hline 54.050 & 67340 & 58.077 & 17894 & 70.495 & 940 & 84.406 & 666 \\
\hline 54.322 & 672 & 58.115 & 1895 & 70.698 & 2151 & 84.428 & 1292 \\
\hline 54.367 & 512 & 58.338 & 1240 & 70.773 & 549 & 84.690 & 638 \\
\hline 54.545 & 557 & 58.368 & 1299 & 70.939 & 1145 & 84.739 & 507 \\
\hline 54.577 & 408 & 58.545 & 508 & 70.964 & 1180 & 85.036 & 526 \\
\hline 54.601 & 541 & 58.597 & 848 & 70.996 & 981 & 85.105 & 81797 \\
\hline 54.656 & 400 & 58.626 & 717 & 71.030 & 371 & 85.440 & 541 \\
\hline 54.790 & 530 & 58.844 & 561 & 71.052 & 494 & 86.109 & 5651 \\
\hline 54.817 & 462 & 58.891 & 446 & 71.089 & 171678 & 87.049 & 290 \\
\hline 54.856 & 590 & 59.051 & 455 & 71.394 & 702 & 89.045 & 307 \\
\hline 54.936 & 510 & 59.081 & 715 & 71.428 & 1183 & 90.050 & 337 \\
\hline 54.959 & 490 & 59.136 & 411 & 71.476 & 974 & 91.059 & 4763 \\
\hline 54.993 & 979 & 60.025 & 2027 & 71.505 & 687 & 92.065 & 976 \\
\hline 55.021 & 1961 & 61.032 & 1152 & 71.647 & 307 & 93.074 & 1509 \\
\hline 55.058 & 469502 & 63.027 & 753 & 71.696 & 787 & 94.081 & 867 \\
\hline 55.083 & 643 & 64.033 & 220 & 71.729 & 775 & 95.090 & 13189 \\
\hline 55.098 & 4011 & 65.043 & 6746 & 71.962 & 501 & 95.163 & 448 \\
\hline 55.301 & 3128 & 66.050 & 5871 & 71.995 & 392 & 96.098 & 34487 \\
\hline 55.326 & 2767 & 67.058 & 79610 & 72.024 & 432 & 96.440 & 430 \\
\hline 55.354 & 4291 & 67.354 & 656 & 72.093 & 9262 & 96.500 & 461 \\
\hline 55.383 & 3383 & 67.409 & 472 & 73.033 & 1412 & 96.802 & 428 \\
\hline 55.407 & 948 & 67.639 & 511 & 73.092 & 410 & 97.075 & 952 \\
\hline 55.426 & 1023 & 67.672 & 489 & 74.039 & 317 & 97.105 & 261753 \\
\hline 55.582 & 3420 & 67.894 & 471 & 77.043 & 4648 & 97.425 & 1642 \\
\hline 55.615 & 3296 & 67.949 & 276 & 78.050 & 1671 & 97.461 & 1612 \\
\hline 55.796 & 1994 & 68.066 & 64040 & 79.059 & 9090 & 97.491 & 2495 \\
\hline 55.816 & 1771 & 68.377 & 464 & 80.065 & 2657 & 97.528 & 2228 \\
\hline 55.846 & 2580 & 68.410 & 537 & 81.074 & 35343 & 97.592 & 554 \\
\hline 56.029 & 1447 & 68.654 & 367 & 81.143 & 692 & 97.816 & 2342 \\
\hline 56.066 & 277472 & 68.685 & 555 & 81.376 & 394 & 98.112 & 82832 \\
\hline 56.093 & 719 & 68.921 & 455 & 82.082 & 79740 & 98.187 & 3466 \\
\hline 56.106 & 2521 & 68.964 & 363 & 82.410 & 525 & 98.378 & 345 \\
\hline 56.299 & 1677 & 68.989 & 363 & 82.465 & 483 & 98.472 & 1832 \\
\hline 56.315 & 2085 & 69.046 & 637 & 82.500 & 435 & 98.512 & 1598 \\
\hline 56.336 & 2205 & 69.074 & 346132 & 82.727 & 445 & 98.796 & 1248 \\
\hline 56.364 & 3212 & 69.118 & 2635 & 82.768 & 402 & 99.119 & 12710 \\
\hline 56.435 & 318 & 69.348 & 1733 & 82.971 & 449 & 99.165 & 557 \\
\hline 56.601 & 2662 & 69.374 & 1856 & 83.002 & 273 & 99.189 & 648 \\
\hline 56.829 & 2072 & 69.407 & 3101 & 83.059 & 1271 & 99.236 & 183 \\
\hline 57.039 & 1679 & 69.439 & 2257 & 83.090 & 349246 & 99.444 & 507 \\
\hline 57.074 & 386362 & 69.464 & 717 & 83.388 & 2072 & 99.475 & 343 \\
\hline 57.101 & 687 & 69.487 & 932 & 83.419 & 1962 & 99.510 & 465 \\
\hline 57.114 & 2950 & 69.682 & 2677 & 83.453 & 3246 & 100.124 & 1179 \\
\hline 57.322 & 3116 & 69.898 & 1295 & 83.487 & 2395 & 105.037 & 441 \\
\hline 57.347 & 2445 & 69.936 & 1939 & 83.542 & 631 & 105.073 & 259 \\
\hline 57.376 & 4165 & 69.979 & 1513 & 83.746 & 2542 & 107.089 & 398 \\
\hline 57.428 & 662 & 70.044 & 1035 & 83.999 & 1534 & 109.106 & 4539 \\
\hline 57.447 & 659 & 70.081 & 289485 & 84.026 & 1479 & 110.113 & 13490 \\
\hline 57.615 & 3482 & 70.360 & 936 & 84.060 & 1831 & 110.193 & 404 \\
\hline
\end{tabular}


Table S5 (continued). High-resolution mass table for the compound identified as hexadecene.

\begin{tabular}{|c|c|c|c|c|c|c|c|}
\hline Mass & $\begin{array}{c}\text { Ion } \\
\text { counts }\end{array}$ & Mass & $\begin{array}{c}\text { Ion } \\
\text { counts }\end{array}$ & Mass & $\begin{array}{c}\text { Ion } \\
\text { counts }\end{array}$ & Mass & $\begin{array}{c}\text { Ion } \\
\text { counts }\end{array}$ \\
\hline 111.121 & 102881 & 113.134 & 5945 & 138.145 & 3338 & 182.208 & 2038 \\
\hline 111.463 & 480 & 113.215 & 508 & 139.153 & 8774 & 183.209 & 420 \\
\hline 111.503 & 989 & 113.256 & 505 & 140.160 & 10894 & 194.208 & 410 \\
\hline 111.560 & 803 & 118.042 & 302 & 141.164 & 1637 & 195.216 & 1399 \\
\hline 111.804 & 310 & 122.043 & 390 & 152.160 & 1998 & 196.223 & 4235 \\
\hline 111.860 & 731 & 123.122 & 1639 & 153.168 & 3737 & 197.226 & 534 \\
\hline 111.901 & 477 & 124.129 & 6297 & 154.176 & 7583 & 222.243 & 307 \\
\hline 111.945 & 538 & 125.137 & 33293 & 155.181 & 1100 & 224.254 & 6023 \\
\hline 112.128 & 42554 & 125.225 & 1062 & 166.176 & 990 & 225.257 & 1128 \\
\hline 112.520 & 744 & 126.144 & 21005 & 167.185 & 2034 & 232.940 & 343 \\
\hline 112.571 & 356 & 126.246 & 711 & 168.191 & 3251 & 234.946 & 273 \\
\hline 112.606 & 522 & 127.149 & 2860 & 169.197 & 479 & 249.944 & 385 \\
\hline 112.853 & 542 & 136.057 & 826 & 180.203 & 339 & 251.948 & 697 \\
\hline 112.896 & 366 & 137.136 & 423 & 181.201 & 923 & & \\
\hline
\end{tabular}


Table S6. High-resolution mass table for the compound identified as hexadecane.

\begin{tabular}{|c|c|c|c|c|c|c|c|}
\hline Mass & $\begin{array}{c}\text { Ion } \\
\text { counts }\end{array}$ & Mass & $\begin{array}{c}\text { Ion } \\
\text { counts }\end{array}$ & Mass & $\begin{array}{c}\text { Ion } \\
\text { counts }\end{array}$ & Mass & $\begin{array}{c}\text { Ion } \\
\text { counts }\end{array}$ \\
\hline 50.018 & 432 & 58.576 & 414 & 73.032 & 972 & 100.125 & 1406 \\
\hline 51.026 & 763 & 58.602 & 339 & 77.042 & 1013 & 105.038 & 283 \\
\hline 52.034 & 478 & 58.626 & 371 & 78.050 & 724 & 110.114 & 913 \\
\hline 53.042 & 4206 & 59.081 & 401 & 79.058 & 1198 & 111.121 & 6058 \\
\hline 54.050 & 5447 & 60.025 & 958 & 80.065 & 319 & 112.129 & 5799 \\
\hline 55.022 & 774 & 61.032 & 1122 & 81.074 & 2629 & 113.136 & 8180 \\
\hline 55.058 & 53417 & 63.028 & 329 & 82.082 & 4902 & 114.140 & 638 \\
\hline 55.325 & 379 & 65.042 & 1442 & 83.089 & 20008 & 122.038 & 233 \\
\hline 55.347 & 334 & 66.049 & 642 & 83.160 & 429 & 124.129 & 325 \\
\hline 56.066 & 35507 & 67.058 & 6391 & 84.097 & 15701 & 125.136 & 2163 \\
\hline 56.624 & 373 & 68.066 & 5407 & 84.164 & 431 & 126.144 & 3661 \\
\hline 57.039 & 732 & 69.074 & 27726 & 85.105 & 80057 & 127.152 & 4717 \\
\hline 57.074 & 217149 & 69.132 & 652 & 85.405 & 415 & 128.155 & 666 \\
\hline 57.322 & 1482 & 70.045 & 658 & 85.440 & 398 & 136.055 & 723 \\
\hline 57.347 & 1686 & 70.081 & 27618 & 85.483 & 691 & 139.154 & 490 \\
\hline 57.375 & 2099 & 70.132 & 376 & 85.521 & 460 & 140.161 & 2523 \\
\hline 57.406 & 1605 & 70.158 & 538 & 85.740 & 463 & 141.168 & 3068 \\
\hline 57.447 & 488 & 71.052 & 380 & 85.789 & 456 & 142.171 & 415 \\
\hline 57.580 & 1116 & 71.089 & 127043 & 86.034 & 439 & 154.176 & 1607 \\
\hline 57.616 & 1731 & 71.365 & 516 & 86.108 & 5320 & 155.183 & 2119 \\
\hline 57.644 & 1534 & 71.394 & 663 & 89.041 & 332 & 168.192 & 1107 \\
\hline 57.834 & 926 & 71.417 & 774 & 90.054 & 259 & 169.200 & 1525 \\
\hline 57.862 & 1081 & 71.442 & 476 & 91.058 & 3217 & 182.209 & 296 \\
\hline 57.884 & 964 & 71.470 & 673 & 92.065 & 437 & 183.215 & 1097 \\
\hline 58.045 & 469 & 71.683 & 613 & 95.090 & 1023 & 196.222 & 487 \\
\hline 58.077 & 10094 & 71.710 & 581 & 96.097 & 2458 & 197.228 & 482 \\
\hline 58.123 & 882 & 71.754 & 395 & 97.105 & 14435 & 226.271 & 1085 \\
\hline 58.168 & 354 & 71.939 & 332 & 98.112 & 9349 & 251.945 & 418 \\
\hline 58.321 & 397 & 71.982 & 526 & 98.185 & 354 & & \\
\hline 58.354 & 538 & 72.093 & 6682 & 99.121 & 16447 & & \\
\hline 58.384 & 476 & 72.213 & 397 & 99.202 & 420 & & \\
\hline & & & & & & & \\
\hline
\end{tabular}


Table S7. High-resolution mass table for the compound identified as octadecene.

\begin{tabular}{|c|c|c|c|c|c|c|c|c|c|}
\hline Mass & $\begin{array}{c}\text { Ion } \\
\text { counts }\end{array}$ & Mass & $\begin{array}{c}\text { Ion } \\
\text { counts }\end{array}$ & Mass & $\begin{array}{c}\text { Ion } \\
\text { counts }\end{array}$ & Mass & $\begin{array}{c}\text { Ion } \\
\text { counts }\end{array}$ & Mass & $\begin{array}{c}\text { Ion } \\
\text { counts }\end{array}$ \\
\hline 50.018 & 1620 & 57.587 & 4085 & 68.936 & 642 & 78.050 & 2077 & 92.065 & 689 \\
\hline 51.026 & 2998 & 57.617 & 4545 & 68.982 & 679 & 79.058 & 11439 & 93.073 & 2272 \\
\hline 52.034 & 2599 & 57.846 & 3358 & 69.037 & 837 & 80.065 & 4168 & 94.082 & 1835 \\
\hline 53.005 & 371 & 57.876 & 2316 & 69.074 & 455523 & 81.038 & 267 & 95.054 & 517 \\
\hline 53.042 & 33699 & 57.896 & 2085 & 69.118 & 3414 & 81.074 & 50532 & 95.090 & 21305 \\
\hline 53.098 & 783 & 58.046 & 1360 & 69.348 & 2303 & 81.358 & 426 & 95.167 & 595 \\
\hline 53.332 & 431 & 58.077 & 25263 & 69.374 & 2354 & 81.398 & 641 & 96.061 & 571 \\
\hline 54.015 & 454 & 58.105 & 1098 & 69.398 & 3030 & 81.449 & 505 & 96.097 & 55859 \\
\hline 54.050 & 80943 & 58.120 & 1825 & 69.427 & 3635 & 81.744 & 422 & 96.462 & 528 \\
\hline 54.108 & 2378 & 58.140 & 2465 & 69.464 & 847 & 82.051 & 741 & 96.500 & 561 \\
\hline 54.294 & 494 & 58.344 & 2169 & 69.486 & 903 & 82.081 & 120425 & 96.790 & 532 \\
\hline 54.316 & 482 & 58.375 & 1979 & 69.639 & 2422 & 82.386 & 788 & 96.845 & 316 \\
\hline 54.341 & 503 & 58.564 & 879 & 69.673 & 3388 & 82.408 & 638 & 96.877 & 417 \\
\hline 54.369 & 492 & 58.607 & 1482 & 69.941 & 2477 & 82.431 & 948 & 97.035 & 648 \\
\hline 54.562 & 442 & 58.633 & 1137 & 69.990 & 1791 & 82.481 & 641 & 97.069 & 2251 \\
\hline 54.598 & 478 & 58.668 & 418 & 70.045 & 1850 & 82.665 & 493 & 97.105 & 402784 \\
\hline 54.842 & 487 & 58.788 & 411 & 70.081 & 319494 & 82.724 & 801 & 97.157 & 3082 \\
\hline 54.870 & 355 & 58.851 & 867 & 70.357 & 1145 & 82.764 & 624 & 97.428 & 2702 \\
\hline 54.961 & 444 & 58.893 & 422 & 70.384 & 1476 & 82.991 & 800 & 97.462 & 2441 \\
\hline 54.985 & 342 & 59.053 & 795 & 70.419 & 2858 & 83.053 & 2775 & 97.498 & 4154 \\
\hline 55.021 & 30119 & 59.080 & 927 & 70.448 & 2069 & 83.089 & 495749 & 97.567 & 837 \\
\hline 55.058 & 549405 & 59.132 & 500 & 70.470 & 1479 & 83.138 & 4176 & 97.593 & 848 \\
\hline 55.083 & 1352 & 59.320 & 418 & 70.496 & 1256 & 83.388 & 3001 & 97.801 & 3204 \\
\hline 55.096 & 11853 & 59.350 & 464 & 70.677 & 1953 & 83.419 & 2452 & 97.842 & 3090 \\
\hline 55.128 & 12258 & 59.584 & 416 & 70.712 & 2172 & 83.447 & 4124 & 98.077 & 4003 \\
\hline 55.174 & 3906 & 60.024 & 3627 & 70.917 & 741 & 83.484 & 3883 & 98.112 & 111905 \\
\hline 55.303 & 3473 & 61.032 & 1645 & 70.958 & 1548 & 83.516 & 1213 & 98.189 & 4712 \\
\hline 55.326 & 3338 & 62.019 & 346 & 70.997 & 1528 & 83.541 & 962 & 98.435 & 1999 \\
\hline 55.354 & 5455 & 63.027 & 597 & 71.051 & 1374 & 83.738 & 3801 & 98.469 & 1636 \\
\hline 55.408 & 1074 & 64.036 & 285 & 71.089 & 257361 & 84.018 & 2785 & 98.502 & 2271 \\
\hline 55.423 & 1067 & 65.042 & 6840 & 71.365 & 823 & 84.060 & 4253 & 98.796 & 1909 \\
\hline 55.585 & 4649 & 66.050 & 7235 & 71.394 & 1202 & 84.097 & 196515 & 99.048 & 1684 \\
\hline 55.835 & 3392 & 67.058 & 100977 & 71.424 & 1748 & 84.346 & 1703 & 99.119 & 22280 \\
\hline 56.027 & 3586 & 67.353 & 717 & 71.454 & 1913 & 84.397 & 1930 & 99.168 & 1329 \\
\hline 56.065 & 332742 & 67.386 & 716 & 71.508 & 810 & 84.428 & 1532 & 99.401 & 564 \\
\hline 56.091 & 852 & 67.413 & 590 & 71.701 & 1585 & 84.460 & 1179 & 99.440 & 1002 \\
\hline 56.310 & 3608 & 67.585 & 325 & 71.964 & 788 & 84.681 & 918 & 99.485 & 794 \\
\hline 56.336 & 2675 & 67.621 & 507 & 71.991 & 477 & 84.731 & 630 & 99.529 & 393 \\
\hline 56.360 & 3997 & 67.647 & 474 & 72.024 & 893 & 84.768 & 563 & 99.720 & 471 \\
\hline 56.435 & 475 & 67.674 & 382 & 72.060 & 591 & 85.032 & 1980 & 99.762 & 541 \\
\hline 56.598 & 3055 & 67.700 & 443 & 72.093 & 13710 & 85.068 & 852 & 99.799 & 410 \\
\hline 56.818 & 2525 & 67.858 & 408 & 72.224 & 437 & 85.105 & 137138 & 99.825 & 364 \\
\hline 56.849 & 2549 & 67.906 & 569 & 72.262 & 520 & 85.411 & 581 & 100.028 & 368 \\
\hline 57.037 & 3364 & 67.939 & 452 & 72.482 & 414 & 85.441 & 1166 & 100.056 & 558 \\
\hline 57.073 & 534996 & 68.066 & 89315 & 72.517 & 429 & 85.520 & 638 & 100.095 & 466 \\
\hline 57.139 & 13222 & 68.366 & 563 & 73.032 & 18533 & 85.735 & 523 & 100.123 & 1799 \\
\hline 57.322 & 4188 & 68.423 & 828 & 73.070 & 508 & 85.791 & 483 & 100.443 & 459 \\
\hline 57.347 & 3574 & 68.640 & 570 & 73.095 & 946 & 86.026 & 405 & 100.769 & 372 \\
\hline 57.362 & 2130 & 68.678 & 630 & 73.345 & 363 & 86.063 & 494 & 101.065 & 653 \\
\hline 57.382 & 5302 & 68.709 & 417 & 73.367 & 374 & 86.108 & 8770 & 105.040 & 287 \\
\hline 57.427 & 1136 & 68.741 & 368 & 74.039 & 1536 & 87.048 & 1019 & 105.075 & 709 \\
\hline 57.447 & 952 & 68.896 & 496 & 77.042 & 5309 & 91.058 & 3138 & 106.074 & 395 \\
\hline
\end{tabular}


Table S7 (continued). High-resolution mass table for the compound identified as octadecene.

\begin{tabular}{|c|c|c|c|c|c|c|c|c|c|}
\hline Mass & $\begin{array}{c}\text { Ion } \\
\text { counts }\end{array}$ & Mass & $\begin{array}{c}\text { Ion } \\
\text { counts }\end{array}$ & Mass & $\begin{array}{c}\text { Ion } \\
\text { counts }\end{array}$ & Mass & $\begin{array}{c}\text { Ion } \\
\text { counts }\end{array}$ & Mass & $\begin{array}{c}\text { Ion } \\
\text { counts }\end{array}$ \\
\hline 107.087 & 816 & 112.875 & 743 & 123.122 & 3447 & 140.160 & 19685 & 183.211 & 896 \\
\hline 108.097 & 570 & 112.902 & 428 & 124.129 & 10638 & 140.240 & 484 & 185.160 & 481 \\
\hline 109.069 & 397 & 112.929 & 690 & 125.105 & 885 & 141.089 & 424 & 194.208 & 528 \\
\hline 109.105 & 7726 & 113.064 & 2377 & 125.136 & 60868 & 141.165 & 3283 & 195.214 & 1155 \\
\hline 110.077 & 650 & 113.135 & 10370 & 125.541 & 439 & 143.106 & 352 & 196.222 & 2578 \\
\hline 110.113 & 21863 & 113.205 & 774 & 125.594 & 488 & 151.152 & 477 & 197.225 & 407 \\
\hline 110.185 & 507 & 113.235 & 646 & 125.990 & 379 & 152.159 & 3535 & 208.221 & 468 \\
\hline 111.084 & 1184 & 113.279 & 552 & 126.144 & 32821 & 153.168 & 7134 & 209.230 & 531 \\
\hline 111.121 & 167172 & 113.517 & 442 & 127.079 & 2433 & 154.175 & 10947 & 210.238 & 1495 \\
\hline 111.469 & 794 & 113.582 & 458 & 127.150 & 5614 & 154.253 & 417 & 211.243 & 318 \\
\hline 111.504 & 997 & 113.632 & 481 & 128.078 & 746 & 155.180 & 1910 & 222.245 & 319 \\
\hline 111.541 & 1597 & 114.071 & 739 & 128.156 & 405 & 160.118 & 317 & 223.245 & 468 \\
\hline 111.858 & 1181 & 114.139 & 662 & 129.095 & 1357 & 166.176 & 2404 & 224.253 & 4233 \\
\hline 111.893 & 1158 & 115.067 & 1072 & 136.081 & 1081 & 167.183 & 3847 & 225.254 & 709 \\
\hline 112.128 & 61102 & 117.075 & 407 & 137.090 & 513 & 168.191 & 7195 & 234.943 & 354 \\
\hline 112.181 & 1215 & 119.089 & 314 & 137.137 & 1699 & 169.196 & 1332 & 249.943 & 411 \\
\hline 112.511 & 681 & 121.101 & 595 & 138.144 & 6279 & 180.191 & 1329 & 251.945 & 604 \\
\hline 112.543 & 742 & 122.110 & 399 & 139.152 & 17340 & 181.199 & 2158 & 252.285 & 3256 \\
\hline 112.591 & 1063 & 123.085 & 416 & 139.243 & 514 & 182.206 & 5203 & 253.288 & 767 \\
\hline
\end{tabular}


Table S8. High-resolution mass table for the compound identified as tetradecanoic acid.

\begin{tabular}{|c|c|c|c|c|c|c|c|}
\hline Mass & $\begin{array}{c}\text { Ion } \\
\text { counts }\end{array}$ & Mass & $\begin{array}{c}\text { Ion } \\
\text { counts }\end{array}$ & Mass & $\begin{array}{c}\text { Ion } \\
\text { counts }\end{array}$ & Mass & $\begin{array}{c}\text { Ion } \\
\text { counts }\end{array}$ \\
\hline 50.019 & 1314 & 60.554 & 1340 & 73.626 & 1421 & 95.053 & 1606 \\
\hline 51.026 & 2972 & 60.588 & 1537 & 73.657 & 1684 & 95.089 & 9919 \\
\hline 52.034 & 1726 & 60.813 & 846 & 73.899 & 979 & 96.061 & 3705 \\
\hline 53.006 & 209 & 60.874 & 636 & 73.930 & 1020 & 96.097 & 8828 \\
\hline 53.042 & 11855 & 61.032 & 40961 & 73.960 & 756 & 97.069 & 15910 \\
\hline 54.050 & 15368 & 61.097 & 1790 & 74.038 & 17909 & 97.105 & 21253 \\
\hline 54.112 & 441 & 61.316 & 891 & 74.073 & 303 & 97.191 & 656 \\
\hline 55.021 & 21587 & 61.349 & 667 & 74.113 & 447 & 98.076 & 21813 \\
\hline 55.058 & 132368 & 61.374 & 650 & 74.149 & 316 & 98.112 & 6685 \\
\hline 55.301 & 810 & 61.549 & 466 & 74.188 & 736 & 98.158 & 678 \\
\hline 55.327 & 1014 & 61.584 & 487 & 74.218 & 499 & 99.049 & 1646 \\
\hline 55.346 & 1016 & 61.617 & 352 & 74.260 & 406 & 99.082 & 2414 \\
\hline 55.374 & 1216 & 61.640 & 352 & 74.449 & 430 & 99.120 & 12132 \\
\hline 55.544 & 553 & 61.827 & 382 & 74.492 & 466 & 99.176 & 421 \\
\hline 55.579 & 1051 & 61.849 & 328 & 74.754 & 402 & 100.056 & 2053 \\
\hline 55.610 & 761 & 61.876 & 432 & 75.037 & 1989 & 100.090 & 456 \\
\hline 55.828 & 683 & 62.032 & 2324 & 76.036 & 395 & 100.125 & 1116 \\
\hline 56.028 & 2686 & 62.067 & 390 & 77.042 & 5503 & 101.063 & 14139 \\
\hline 56.065 & 37019 & 62.135 & 390 & 78.049 & 2633 & 101.101 & 523 \\
\hline 56.101 & 739 & 63.027 & 1096 & 79.058 & 8841 & 101.149 & 384 \\
\hline 56.123 & 1297 & 65.042 & 3201 & 80.065 & 4049 & 102.071 & 3795 \\
\hline 56.305 & 448 & 66.050 & 2215 & 81.037 & 915 & 103.069 & 852 \\
\hline 56.341 & 664 & 67.058 & 22322 & 81.074 & 17092 & 104.065 & 392 \\
\hline 56.594 & 447 & 67.126 & 508 & 81.124 & 339 & 105.038 & 922 \\
\hline 57.037 & 6297 & 68.029 & 712 & 82.045 & 1109 & 105.073 & 1722 \\
\hline 57.073 & 134553 & 68.065 & 16018 & 82.081 & 13325 & 106.045 & 504 \\
\hline 57.323 & 846 & 69.037 & 2748 & 82.145 & 425 & 106.080 & 534 \\
\hline 57.346 & 844 & 69.073 & 80682 & 83.053 & 16252 & 107.053 & 7917 \\
\hline 57.373 & 1195 & 69.375 & 484 & 83.089 & 39147 & 107.089 & 2502 \\
\hline 57.409 & 631 & 69.413 & 534 & 83.166 & 1163 & 108.057 & 863 \\
\hline 57.578 & 553 & 69.685 & 518 & 83.418 & 481 & 108.097 & 1287 \\
\hline 57.608 & 760 & 69.938 & 409 & 83.459 & 308 & 109.069 & 1570 \\
\hline 57.630 & 621 & 70.045 & 3723 & 84.061 & 17240 & 109.105 & 3599 \\
\hline 57.664 & 474 & 70.081 & 24060 & 84.096 & 11338 & 110.077 & 2324 \\
\hline 57.814 & 421 & 71.053 & 6113 & 84.136 & 531 & 110.113 & 4321 \\
\hline 57.846 & 465 & 71.089 & 65232 & 85.032 & 1006 & 111.048 & 332 \\
\hline 57.877 & 548 & 71.156 & 1673 & 85.067 & 3727 & 111.084 & 7878 \\
\hline 58.045 & 6317 & 71.395 & 372 & 85.105 & 41754 & 111.121 & 7928 \\
\hline 58.077 & 6288 & 71.421 & 345 & 86.040 & 1390 & 111.172 & 375 \\
\hline 58.127 & 563 & 71.445 & 349 & 86.074 & 566 & 112.091 & 4754 \\
\hline 58.346 & 464 & 71.468 & 392 & 86.108 & 2873 & 112.128 & 3162 \\
\hline 58.373 & 403 & 72.025 & 398 & 87.048 & 33735 & 113.064 & 1302 \\
\hline 59.053 & 10685 & 72.059 & 776 & 87.084 & 1615 & 113.097 & 816 \\
\hline 59.138 & 395 & 72.093 & 3660 & 87.121 & 970 & 113.136 & 7068 \\
\hline 60.005 & 359 & 73.003 & 407 & 88.054 & 3590 & 114.072 & 2012 \\
\hline 60.024 & 198821 & 73.032 & 221507 & 89.055 & 906 & 114.140 & 667 \\
\hline 60.065 & 1976 & 73.069 & 8801 & 91.058 & 3988 & 115.079 & 22406 \\
\hline 60.280 & 996 & 73.310 & 1372 & 92.065 & 1006 & 115.167 & 611 \\
\hline 60.304 & 1251 & 73.341 & 1254 & 93.042 & 333 & 116.086 & 5350 \\
\hline 60.328 & 1722 & 73.367 & 2188 & 93.074 & 4322 & 117.076 & 913 \\
\hline 60.355 & 1729 & 73.402 & 1865 & 94.042 & 538 & 119.089 & 780 \\
\hline 60.405 & 325 & 73.456 & 437 & 94.081 & 2660 & 120.096 & 307 \\
\hline
\end{tabular}


Table S8 (continued). High-resolution mass table for the compound identified as tetradecanoic acid.

\begin{tabular}{|c|c|c|c|c|c|c|c|}
\hline Mass & $\begin{array}{c}\text { Ion } \\
\text { counts }\end{array}$ & Mass & $\begin{array}{c}\text { Ion } \\
\text { counts }\end{array}$ & Mass & $\begin{array}{c}\text { Ion } \\
\text { counts }\end{array}$ & Mass & $\begin{array}{c}\text { Ion } \\
\text { counts }\end{array}$ \\
\hline 121.032 & 405 & 130.099 & 6680 & 148.128 & 382 & 172.147 & 1910 \\
\hline 121.105 & 2706 & 131.098 & 904 & 149.136 & 1086 & 179.149 & 241 \\
\hline 122.041 & 603 & 133.104 & 794 & 151.117 & 627 & 180.191 & 950 \\
\hline 122.078 & 328 & 134.112 & 422 & 152.051 & 2248 & 185.157 & 42683 \\
\hline 122.111 & 668 & 135.121 & 1837 & 152.118 & 416 & 185.262 & 1449 \\
\hline 123.084 & 2224 & 136.055 & 463 & 152.162 & 369 & 185.645 & 493 \\
\hline 123.121 & 1520 & 136.089 & 313 & 153.064 & 385 & 186.161 & 5725 \\
\hline 124.091 & 1129 & 136.123 & 402 & 153.132 & 744 & 187.166 & 514 \\
\hline 124.128 & 3020 & 137.100 & 1104 & 157.126 & 5430 & 192.190 & 617 \\
\hline 125.100 & 3522 & 137.137 & 781 & 158.130 & 944 & 199.173 & 7868 \\
\hline 125.136 & 3374 & 138.107 & 782 & 163.152 & 560 & 200.178 & 1434 \\
\hline 126.106 & 835 & 138.144 & 2723 & 164.159 & 1061 & 208.186 & 968 \\
\hline 126.144 & 2019 & 139.115 & 1649 & 165.132 & 358 & 209.194 & 435 \\
\hline 127.078 & 1086 & 139.151 & 1071 & 166.175 & 5822 & 211.209 & 710 \\
\hline 127.112 & 288 & 140.122 & 500 & 167.146 & 720 & 218.958 & 345 \\
\hline 127.152 & 2865 & 140.160 & 1803 & 167.179 & 963 & 228.211 & 9343 \\
\hline 128.065 & 656 & 143.110 & 13214 & 168.093 & 517 & 229.214 & 1499 \\
\hline 128.148 & 342 & 143.179 & 398 & 168.191 & 2027 & 234.946 & 372 \\
\hline 129.095 & 68939 & 144.114 & 1509 & 169.198 & 1042 & 249.943 & 529 \\
\hline 129.516 & 576 & 145.114 & 324 & 171.142 & 12726 & 251.946 & 914 \\
\hline 129.931 & 450 & 147.119 & 423 & 171.246 & 427 & & \\
\hline
\end{tabular}


Table S9. High-resolution mass table for the compound identified as pentadecanoic acid.

\begin{tabular}{|c|c|c|c|c|c|c|c|c|c|}
\hline Mass & $\begin{array}{c}\text { Ion } \\
\text { counts }\end{array}$ & Mass & $\begin{array}{c}\text { Ion } \\
\text { counts }\end{array}$ & Mass & $\begin{array}{c}\text { Ion } \\
\text { counts }\end{array}$ & Mass & $\begin{array}{c}\text { Ion } \\
\text { counts }\end{array}$ & Mass & $\begin{array}{c}\text { Ion } \\
\text { counts }\end{array}$ \\
\hline 50.018 & 1337 & 59.839 & 384 & 70.045 & 4754 & 79.058 & 12024 & 97.185 & 1524 \\
\hline 51.026 & 2554 & 59.898 & 416 & 70.081 & 36064 & 80.065 & 5582 & 97.433 & 478 \\
\hline 52.034 & 1716 & 60.003 & 397 & 70.382 & 256 & 81.038 & 1029 & 97.472 & 574 \\
\hline 53.042 & 16063 & 60.024 & 276009 & 70.491 & 326 & 81.073 & 25616 & 97.778 & 441 \\
\hline 54.050 & 21503 & 60.278 & 1704 & 71.021 & 383 & 81.147 & 758 & 98.076 & 34400 \\
\hline 54.106 & 523 & 60.304 & 1733 & 71.053 & 7532 & 82.045 & 1442 & 98.112 & 10437 \\
\hline 55.021 & 27795 & 60.331 & 2728 & 71.089 & 106999 & 82.081 & 20974 & 98.158 & 1305 \\
\hline 55.058 & 196295 & 60.408 & 876 & 71.158 & 2883 & 82.150 & 497 & 98.469 & 544 \\
\hline 55.097 & 1882 & 60.556 & 2000 & 71.197 & 1015 & 83.053 & 22955 & 98.767 & 443 \\
\hline 55.300 & 1426 & 60.588 & 2295 & 71.358 & 400 & 83.089 & 67181 & 99.048 & 2500 \\
\hline 55.325 & 1317 & 60.749 & 270 & 71.395 & 517 & 83.136 & 1084 & 99.081 & 4223 \\
\hline 55.354 & 1944 & 60.819 & 1612 & 71.421 & 709 & 83.165 & 2265 & 99.120 & 16421 \\
\hline 55.406 & 368 & 60.860 & 1482 & 71.464 & 502 & 83.388 & 577 & 99.176 & 418 \\
\hline 55.426 & 302 & 61.031 & 63127 & 71.670 & 570 & 83.421 & 584 & 100.055 & 2616 \\
\hline 55.574 & 1591 & 61.095 & 2813 & 71.708 & 655 & 83.458 & 394 & 100.089 & 701 \\
\hline 55.819 & 1195 & 61.293 & 756 & 71.952 & 354 & 83.493 & 472 & 100.125 & 1488 \\
\hline 55.852 & 1055 & 61.318 & 1282 & 72.024 & 315 & 83.721 & 502 & 101.063 & 25085 \\
\hline 56.028 & 3323 & 61.344 & 1477 & 72.059 & 1062 & 84.060 & 25799 & 101.101 & 901 \\
\hline 56.065 & 56130 & 61.369 & 1138 & 72.092 & 5674 & 84.096 & 18172 & 101.144 & 606 \\
\hline 56.282 & 465 & 61.549 & 599 & 72.232 & 438 & 84.136 & 828 & 102.070 & 6601 \\
\hline 56.315 & 803 & 61.570 & 606 & 72.721 & 342 & 84.172 & 576 & 103.070 & 951 \\
\hline 56.334 & 829 & 61.597 & 922 & 72.764 & 412 & 84.399 & 483 & 104.065 & 554 \\
\hline 56.545 & 537 & 61.640 & 664 & 72.816 & 393 & 85.032 & 1317 & 105.038 & 822 \\
\hline 56.572 & 326 & 61.822 & 518 & 73.010 & 587 & 85.067 & 5249 & 105.073 & 2223 \\
\hline 56.607 & 554 & 61.868 & 687 & 73.032 & 318622 & 85.105 & 64143 & 106.079 & 629 \\
\hline 56.822 & 453 & 62.032 & 2850 & 73.069 & 12839 & 85.438 & 523 & 107.052 & 1048 \\
\hline 56.865 & 422 & 62.085 & 431 & 73.313 & 1936 & 85.730 & 278 & 107.089 & 3726 \\
\hline 57.037 & 9508 & 62.120 & 506 & 73.341 & 1893 & 85.785 & 423 & 108.096 & 2138 \\
\hline 57.073 & 214541 & 62.149 & 369 & 73.372 & 3032 & 86.040 & 1977 & 109.068 & 2089 \\
\hline 57.322 & 1524 & 62.322 & 372 & 73.409 & 2384 & 86.073 & 800 & 109.105 & 6034 \\
\hline 57.346 & 1485 & 62.377 & 386 & 73.454 & 644 & 86.108 & 4478 & 110.076 & 3360 \\
\hline 57.370 & 2039 & 62.406 & 415 & 73.649 & 2589 & 87.048 & 50532 & 110.112 & 6061 \\
\hline 57.448 & 396 & 63.029 & 972 & 73.902 & 1692 & 87.084 & 2901 & 111.084 & 12855 \\
\hline 57.600 & 1670 & 65.042 & 4341 & 73.942 & 1700 & 87.389 & 269 & 111.120 & 13820 \\
\hline 57.807 & 669 & 66.050 & 2937 & 74.038 & 26157 & 87.417 & 405 & 111.176 & 465 \\
\hline 57.844 & 1000 & 67.058 & 32090 & 74.073 & 473 & 87.735 & 335 & 111.203 & 592 \\
\hline 57.869 & 1241 & 68.030 & 1218 & 74.101 & 576 & 88.054 & 6087 & 112.091 & 7328 \\
\hline 57.930 & 332 & 68.065 & 23269 & 74.141 & 659 & 89.058 & 1018 & 112.127 & 5314 \\
\hline 58.044 & 3858 & 68.139 & 472 & 74.183 & 1281 & 91.058 & 4771 & 112.178 & 328 \\
\hline 58.077 & 9494 & 68.409 & 314 & 74.257 & 683 & 92.065 & 1218 & 113.063 & 1763 \\
\hline 58.112 & 951 & 69.037 & 3861 & 74.413 & 399 & 93.073 & 6470 & 113.097 & 1026 \\
\hline 58.146 & 722 & 69.073 & 123459 & 74.475 & 867 & 94.045 & 419 & 113.136 & 10582 \\
\hline 58.302 & 398 & 69.343 & 477 & 74.705 & 324 & 94.081 & 4009 & 114.071 & 3024 \\
\hline 58.339 & 587 & 69.375 & 735 & 74.734 & 460 & 95.053 & 1975 & 114.139 & 1101 \\
\hline 58.373 & 571 & 69.399 & 933 & 74.776 & 417 & 95.089 & 16232 & 115.079 & 33601 \\
\hline 58.568 & 414 & 69.423 & 988 & 74.804 & 350 & 95.142 & 235 & 115.118 & 412 \\
\hline 58.622 & 436 & 69.486 & 369 & 75.037 & 3055 & 96.061 & 5218 & 115.489 & 396 \\
\hline 58.866 & 438 & 69.643 & 763 & 75.074 & 376 & 96.097 & 13505 & 116.086 & 8237 \\
\hline 59.053 & 13900 & 69.684 & 942 & 75.334 & 362 & 96.151 & 356 & 117.081 & 1718 \\
\hline 59.083 & 452 & 69.731 & 392 & 76.042 & 338 & 97.032 & 657 & 118.080 & 468 \\
\hline 59.114 & 741 & 69.916 & 455 & 77.042 & 4753 & 97.068 & 25121 & 119.089 & 1373 \\
\hline 59.612 & 346 & 69.956 & 479 & 78.050 & 2440 & 97.105 & 38637 & 120.094 & 547 \\
\hline
\end{tabular}


Table S9 (continued). High-resolution mass table for the compound identified as pentadecanoic acid.

\begin{tabular}{|c|c|c|c|c|c|c|c|c|c|}
\hline Mass & $\begin{array}{c}\text { Ion } \\
\text { counts }\end{array}$ & Mass & $\begin{array}{c}\text { Ion } \\
\text { counts }\end{array}$ & Mass & $\begin{array}{c}\text { Ion } \\
\text { counts }\end{array}$ & Mass & $\begin{array}{c}\text { Ion } \\
\text { counts }\end{array}$ & Mass & $\begin{array}{c}\text { Ion } \\
\text { counts }\end{array}$ \\
\hline 121.067 & 509 & 130.099 & 8950 & 144.115 & 3659 & 166.174 & 709 & 199.284 & 1594 \\
\hline 121.105 & 4441 & 130.254 & 440 & 145.113 & 766 & 167.148 & 1240 & 200.176 & 7091 \\
\hline 122.041 & 612 & 130.302 & 429 & 147.120 & 624 & 168.152 & 448 & 201.178 & 849 \\
\hline 122.112 & 1244 & 131.100 & 1353 & 149.136 & 2188 & 171.141 & 15890 & 205.197 & 477 \\
\hline 123.084 & 3160 & 133.104 & 996 & 150.144 & 469 & 171.236 & 472 & 206.205 & 707 \\
\hline 123.120 & 2565 & 134.113 & 534 & 151.115 & 868 & 172.145 & 2255 & 213.188 & 10511 \\
\hline 124.092 & 1658 & 135.084 & 399 & 151.153 & 1075 & 177.167 & 1974 & 214.192 & 1863 \\
\hline 124.128 & 4142 & 135.120 & 3183 & 152.063 & 421 & 178.175 & 1159 & 215.200 & 278 \\
\hline 125.063 & 528 & 136.097 & 265 & 152.124 & 448 & 179.146 & 396 & 218.948 & 375 \\
\hline 125.100 & 6164 & 136.128 & 889 & 152.158 & 2881 & 180.190 & 7241 & 222.200 & 1674 \\
\hline 125.136 & 5110 & 137.061 & 384 & 153.067 & 307 & 181.164 & 780 & 223.208 & 425 \\
\hline 126.074 & 479 & 137.100 & 1738 & 153.131 & 1812 & 181.195 & 1101 & 225.225 & 974 \\
\hline 126.106 & 1368 & 137.136 & 1491 & 153.167 & 1333 & 182.206 & 2018 & 227.207 & 605 \\
\hline 126.144 & 2685 & 138.107 & 1285 & 154.175 & 2238 & 183.214 & 1648 & 228.215 & 638 \\
\hline 127.078 & 1491 & 138.143 & 3327 & 155.177 & 358 & 184.219 & 318 & 234.941 & 332 \\
\hline 127.115 & 512 & 139.115 & 3814 & 157.126 & 16599 & 185.157 & 28111 & 240.209 & 279 \\
\hline 127.151 & 6742 & 139.151 & 1858 & 157.223 & 478 & 185.263 & 771 & 242.227 & 15708 \\
\hline 128.075 & 970 & 140.120 & 596 & 158.130 & 2528 & 186.161 & 4405 & 242.364 & 589 \\
\hline 128.155 & 636 & 140.159 & 1659 & 159.133 & 350 & 187.170 & 763 & 243.230 & 2971 \\
\hline 129.094 & 88374 & 141.085 & 616 & 161.134 & 394 & 191.181 & 328 & 244.238 & 418 \\
\hline 129.473 & 428 & 141.167 & 2849 & 163.151 & 1135 & 193.163 & 275 & 249.942 & 591 \\
\hline 129.505 & 517 & 142.173 & 295 & 164.158 & 388 & 194.205 & 767 & 251.946 & 1153 \\
\hline 129.565 & 713 & 143.110 & 27956 & 165.072 & 324 & 195.176 & 1595 & & \\
\hline 129.874 & 512 & 143.206 & 580 & 165.132 & 656 & 199.172 & 48482 & & \\
\hline
\end{tabular}


Table S10. High-resolution mass table for the compound identified as hexadecanoic acid.

\begin{tabular}{|c|c|c|c|c|c|c|c|c|c|}
\hline Mass & $\begin{array}{c}\text { Ion } \\
\text { counts }\end{array}$ & Mass & $\begin{array}{c}\text { Ion } \\
\text { counts }\end{array}$ & Mass & $\begin{array}{c}\text { Ion } \\
\text { counts }\end{array}$ & Mass & $\begin{array}{c}\text { Ion } \\
\text { counts }\end{array}$ & Mass & $\begin{array}{c}\text { Ion } \\
\text { counts }\end{array}$ \\
\hline 50.017 & 590 & 70.045 & 2058 & 93.074 & 3403 & 119.089 & 1062 & 153.130 & 1021 \\
\hline 51.027 & 1361 & 70.081 & 30540 & 94.048 & 351 & 120.094 & 475 & 153.167 & 601 \\
\hline 52.034 & 873 & 70.134 & 574 & 94.081 & 2006 & 121.103 & 3376 & 154.078 & 2417 \\
\hline 53.042 & 6898 & 70.158 & 617 & 95.053 & 1180 & 122.110 & 777 & 154.161 & 423 \\
\hline 54.050 & 10015 & 70.191 & 470 & 95.089 & 12144 & 123.084 & 2240 & 155.092 & 400 \\
\hline 55.021 & 8709 & 71.053 & 3838 & 96.061 & 3533 & 123.121 & 2091 & 155.183 & 373 \\
\hline 55.058 & 75883 & 71.089 & 37157 & 96.096 & 6755 & 124.090 & 1427 & 157.125 & 2908 \\
\hline 55.296 & 349 & 71.157 & 693 & 97.068 & 8838 & 124.127 & 1612 & 158.132 & 1186 \\
\hline 55.326 & 680 & 71.194 & 474 & 97.105 & 22539 & 125.068 & 607 & 159.142 & 352 \\
\hline 55.374 & 432 & 72.059 & 776 & 97.155 & 559 & 125.100 & 3214 & 163.152 & 1354 \\
\hline 55.554 & 379 & 72.092 & 2173 & 97.189 & 700 & 125.136 & 3405 & 165.130 & 586 \\
\hline 55.571 & 317 & 73.032 & 48109 & 98.076 & 9422 & 126.066 & 406 & 167.147 & 1824 \\
\hline 55.595 & 360 & 73.068 & 3205 & 98.111 & 4641 & 126.107 & 817 & 168.149 & 359 \\
\hline 55.619 & 416 & 73.334 & 407 & 98.171 & 381 & 126.143 & 1163 & 168.193 & 246 \\
\hline 56.028 & 1522 & 73.359 & 305 & 99.047 & 1350 & 127.079 & 1317 & 169.078 & 332 \\
\hline 56.065 & 38204 & 73.398 & 401 & 99.082 & 1562 & 127.151 & 2141 & 171.142 & 2156 \\
\hline 56.118 & 1055 & 74.039 & 17215 & 99.120 & 4555 & 128.074 & 586 & 175.151 & 369 \\
\hline 56.334 & 446 & 74.107 & 415 & 100.055 & 1560 & 129.095 & 12560 & 177.168 & 1220 \\
\hline 56.361 & 452 & 75.041 & 1029 & 100.123 & 342 & 129.179 & 387 & 179.152 & 427 \\
\hline 57.037 & 4904 & 77.042 & 2770 & 101.063 & 7955 & 130.101 & 2866 & 180.191 & 744 \\
\hline 57.073 & 80318 & 78.049 & 1442 & 101.103 & 407 & 131.098 & 576 & 181.162 & 1716 \\
\hline 57.323 & 564 & 79.058 & 6814 & 102.069 & 1561 & 133.105 & 1021 & 182.157 & 370 \\
\hline 57.346 & 488 & 80.065 & 3117 & 103.062 & 466 & 134.107 & 524 & 185.157 & 4391 \\
\hline 57.381 & 767 & 81.037 & 574 & 104.066 & 287 & 135.121 & 1793 & 186.162 & 1234 \\
\hline 57.569 & 446 & 81.073 & 17045 & 105.073 & 1555 & 136.129 & 783 & 187.169 & 574 \\
\hline 57.608 & 462 & 81.129 & 357 & 106.080 & 471 & 137.100 & 1535 & 192.190 & 357 \\
\hline 57.632 & 496 & 82.044 & 956 & 107.053 & 665 & 137.136 & 933 & 193.165 & 469 \\
\hline 57.878 & 405 & 82.081 & 12032 & 107.089 & 1795 & 138.108 & 947 & 194.208 & 2329 \\
\hline 58.044 & 1605 & 83.053 & 7829 & 108.097 & 1209 & 138.143 & 2034 & 195.179 & 1439 \\
\hline 58.077 & 3672 & 83.089 & 35285 & 109.069 & 1474 & 139.115 & 4479 & 197.228 & 956 \\
\hline 59.053 & 4360 & 83.166 & 959 & 109.105 & 4627 & 139.152 & 1612 & 199.172 & 6879 \\
\hline 60.024 & 29598 & 83.425 & 300 & 110.076 & 2441 & 140.120 & 783 & 200.177 & 973 \\
\hline 60.091 & 747 & 84.060 & 7556 & 110.112 & 2745 & 140.158 & 747 & 213.189 & 2948 \\
\hline 61.031 & 6438 & 84.096 & 9169 & 111.084 & 6129 & 141.096 & 635 & 214.191 & 498 \\
\hline 63.027 & 281 & 85.032 & 696 & 111.120 & 8553 & 141.167 & 1294 & 220.224 & 379 \\
\hline 65.042 & 2248 & 85.068 & 2612 & 112.091 & 3431 & 143.110 & 4561 & 223.208 & 319 \\
\hline 66.050 & 1384 & 85.105 & 18826 & 112.127 & 2182 & 144.115 & 515 & 226.195 & 327 \\
\hline 67.058 & 18182 & 85.181 & 459 & 113.064 & 1171 & 147.120 & 653 & 227.202 & 971 \\
\hline 67.116 & 428 & 86.041 & 615 & 113.098 & 648 & 148.125 & 274 & 234.940 & 400 \\
\hline 68.030 & 429 & 86.107 & 1947 & 113.136 & 2884 & 149.135 & 2276 & 236.217 & 1032 \\
\hline 68.065 & 13077 & 87.048 & 15157 & 114.070 & 1931 & 150.140 & 571 & 242.227 & 597 \\
\hline 69.037 & 1683 & 87.084 & 719 & 115.079 & 5835 & 151.113 & 687 & 249.946 & 350 \\
\hline 69.073 & 57744 & 87.125 & 443 & 115.118 & 355 & 151.152 & 551 & 251.949 & 560 \\
\hline 69.426 & 382 & 88.053 & 1298 & 116.086 & 1952 & 152.058 & 329 & 254.226 & 448 \\
\hline 69.655 & 424 & 91.058 & 2750 & 117.075 & 588 & 152.121 & 350 & 256.243 & 1237 \\
\hline 69.698 & 345 & 92.065 & 818 & 118.073 & 326 & 152.158 & 630 & & \\
\hline
\end{tabular}


Table S11. High-resolution mass table for the compound identified as hexadecenoic acid.

\begin{tabular}{|c|c|c|c|c|c|c|c|}
\hline Mass & $\begin{array}{c}\text { Ion } \\
\text { counts }\end{array}$ & Mass & $\begin{array}{c}\text { Ion } \\
\text { counts }\end{array}$ & Mass & $\begin{array}{c}\text { Ion } \\
\text { counts }\end{array}$ & Mass & $\begin{array}{c}\text { Ion } \\
\text { counts }\end{array}$ \\
\hline 48.032 & 355 & 56.611 & 3782 & 60.812 & 2209 & 68.452 & 1055 \\
\hline 48.058 & 426 & 56.796 & 2684 & 60.844 & 2307 & 68.475 & 1170 \\
\hline 49.010 & 374 & 56.831 & 3507 & 61.032 & 81064 & 68.672 & 2588 \\
\hline 50.019 & 3506 & 57.037 & 56916 & 61.062 & 925 & 68.702 & 2190 \\
\hline 51.026 & 10830 & 57.073 & 529719 & 61.073 & 1222 & 68.743 & 1003 \\
\hline 52.034 & 8692 & 57.113 & 8662 & 61.291 & 1025 & 68.905 & 1764 \\
\hline 53.005 & 545 & 57.137 & 14024 & 61.318 & 1474 & 68.936 & 2209 \\
\hline 53.042 & 102836 & 57.321 & 5014 & 61.344 & 2024 & 68.965 & 1297 \\
\hline 53.080 & 943 & 57.347 & 3688 & 61.574 & 1411 & 69.002 & 1868 \\
\hline 53.106 & 1695 & 57.376 & 5669 & 61.612 & 1301 & 69.037 & 17242 \\
\hline 53.276 & 479 & 57.427 & 1057 & 61.825 & 789 & 69.074 & 623453 \\
\hline 53.314 & 1327 & 57.447 & 615 & 61.848 & 596 & 69.116 & 25742 \\
\hline 53.538 & 648 & 57.606 & 4932 & 61.874 & 729 & 69.314 & 2148 \\
\hline 53.596 & 639 & 57.837 & 3622 & 62.032 & 3837 & 69.356 & 2916 \\
\hline 53.790 & 513 & 57.868 & 3372 & 62.076 & 621 & 69.374 & 3972 \\
\hline 53.831 & 408 & 58.045 & 14257 & 62.098 & 546 & 69.410 & 6641 \\
\hline 53.873 & 347 & 58.077 & 24883 & 62.123 & 603 & 69.464 & 1775 \\
\hline 54.015 & 1280 & 58.111 & 2716 & 62.325 & 435 & 69.482 & 1902 \\
\hline 54.050 & 254170 & 58.352 & 2372 & 62.361 & 433 & 69.616 & 2045 \\
\hline 54.089 & 2318 & 58.558 & 1289 & 62.396 & 418 & 69.680 & 5361 \\
\hline 54.291 & 1748 & 58.592 & 1648 & 62.600 & 389 & 69.913 & 3296 \\
\hline 54.315 & 1526 & 58.783 & 431 & 62.638 & 411 & 69.937 & 1987 \\
\hline 54.339 & 2311 & 58.844 & 1271 & 63.027 & 3190 & 69.962 & 3431 \\
\hline 54.396 & 343 & 58.888 & 937 & 63.111 & 375 & 70.007 & 1668 \\
\hline 54.412 & 339 & 59.053 & 34743 & 64.038 & 1323 & 70.045 & 13389 \\
\hline 54.559 & 1890 & 59.083 & 770 & 65.042 & 34795 & 70.081 & 327558 \\
\hline 54.799 & 1682 & 59.307 & 609 & 65.311 & 470 & 70.353 & 1037 \\
\hline 54.824 & 1321 & 59.332 & 814 & 65.333 & 513 & 70.384 & 1571 \\
\hline 54.852 & 1191 & 59.362 & 549 & 66.050 & 25974 & 70.412 & 2618 \\
\hline 54.886 & 574 & 59.382 & 692 & 66.118 & 583 & 70.436 & 2006 \\
\hline 54.917 & 355 & 59.548 & 426 & 66.385 & 380 & 70.457 & 2289 \\
\hline 54.980 & 1076 & 59.583 & 724 & 66.866 & 415 & 70.476 & 1067 \\
\hline 55.003 & 882 & 59.618 & 704 & 66.910 & 467 & 70.495 & 1915 \\
\hline 55.021 & 79486 & 59.668 & 409 & 67.030 & 1008 & 70.708 & 2440 \\
\hline 55.059 & 773264 & 59.795 & 507 & 67.058 & 381702 & 70.736 & 2046 \\
\hline 55.097 & 61225 & 59.849 & 962 & 67.087 & 411 & 70.927 & 949 \\
\hline 55.275 & 6121 & 59.880 & 637 & 67.101 & 2707 & 70.951 & 947 \\
\hline 55.312 & 3803 & 59.916 & 417 & 67.329 & 2311 & 70.985 & 1655 \\
\hline 55.326 & 7715 & 59.947 & 467 & 67.354 & 2038 & 71.017 & 2137 \\
\hline 55.362 & 9500 & 60.005 & 616 & 67.391 & 3551 & 71.053 & 65813 \\
\hline 55.406 & 3963 & 60.024 & 383404 & 67.464 & 997 & 71.089 & 242003 \\
\hline 55.425 & 1720 & 60.052 & 1462 & 67.619 & 2558 & 71.118 & 699 \\
\hline 55.585 & 7869 & 60.063 & 3880 & 67.650 & 3114 & 71.133 & 2571 \\
\hline 55.824 & 6193 & 60.278 & 2347 & 67.897 & 2207 & 71.364 & 1218 \\
\hline 56.028 & 12623 & 60.304 & 2371 & 67.928 & 1949 & 71.394 & 1402 \\
\hline 56.065 & 375270 & 60.322 & 1600 & 67.957 & 1777 & 71.423 & 1832 \\
\hline 56.091 & 1566 & 60.339 & 3322 & 68.029 & 5931 & 71.472 & 1430 \\
\hline 56.311 & 5118 & 60.366 & 2613 & 68.065 & 282992 & 71.506 & 709 \\
\hline 56.336 & 3680 & 60.387 & 1056 & 68.108 & 2041 & 71.684 & 1759 \\
\hline 56.359 & 5152 & 60.407 & 1004 & 68.340 & 1278 & 71.719 & 1393 \\
\hline 56.435 & 312 & 60.563 & 3109 & 68.364 & 1652 & 71.913 & 395 \\
\hline 56.572 & 4003 & 60.594 & 3074 & 68.395 & 2694 & 71.941 & 598 \\
\hline
\end{tabular}


Table S11 (continued). High-resolution mass table for the compound identified as hexadecenoic acid.

\begin{tabular}{|c|c|c|c|c|c|c|c|}
\hline Mass & $\begin{array}{c}\begin{array}{c}\text { Ion } \\
\text { counts }\end{array} \\
\end{array}$ & Mass & $\begin{array}{c}\text { Ion } \\
\text { counts }\end{array}$ & Mass & $\begin{array}{c}\text { Ion } \\
\text { counts }\end{array}$ & Mass & $\begin{array}{c}\text { Ion } \\
\text { counts }\end{array}$ \\
\hline 71.971 & 736 & 79.660 & 660 & 84.452 & 2731 & 95.775 & 1921 \\
\hline 72.025 & 1201 & 79.698 & 1011 & 84.514 & 541 & 96.061 & 132919 \\
\hline 72.058 & 5970 & 79.747 & 503 & 84.681 & 2202 & 96.097 & 150408 \\
\hline 72.093 & 12495 & 79.935 & 409 & 84.721 & 2367 & 96.152 & 7297 \\
\hline 72.170 & 403 & 79.977 & 450 & 85.032 & 12315 & 96.417 & 3007 \\
\hline 72.237 & 538 & 80.066 & 82250 & 85.068 & 48982 & 96.452 & 2879 \\
\hline 72.281 & 443 & 80.346 & 497 & 85.105 & 123169 & 96.492 & 2882 \\
\hline 72.318 & 351 & 80.390 & 553 & 85.150 & 2042 & 96.773 & 2814 \\
\hline 72.466 & 470 & 80.447 & 417 & 85.403 & 1053 & 96.815 & 2334 \\
\hline 72.504 & 529 & 80.949 & 448 & 85.468 & 879 & 97.033 & 3897 \\
\hline 72.528 & 419 & 81.037 & 8734 & 85.721 & 495 & 97.069 & 140517 \\
\hline 72.726 & 492 & 81.074 & 395066 & 85.771 & 513 & 97.105 & 315046 \\
\hline 72.750 & 384 & 81.105 & 396 & 86.040 & 8788 & 97.185 & 11065 \\
\hline 72.785 & 550 & 81.369 & 2554 & 86.073 & 4671 & 97.429 & 4328 \\
\hline 72.847 & 509 & 81.399 & 2135 & 86.108 & 8246 & 97.461 & 2790 \\
\hline 72.888 & 533 & 81.427 & 3378 & 87.048 & 114836 & 97.492 & 4599 \\
\hline 72.924 & 490 & 81.462 & 3415 & 87.084 & 7934 & 97.567 & 955 \\
\hline 73.009 & 890 & 81.521 & 948 & 87.346 & 389 & 97.777 & 3785 \\
\hline 73.032 & 461574 & 81.713 & 3076 & 87.385 & 500 & 97.856 & 2028 \\
\hline 73.069 & 32058 & 81.751 & 2800 & 87.412 & 794 & 98.076 & 178969 \\
\hline 73.314 & 2844 & 82.005 & 2477 & 87.670 & 470 & 98.112 & 89485 \\
\hline 73.341 & 2833 & 82.045 & 18338 & 87.730 & 586 & 98.160 & 7306 \\
\hline 73.370 & 4591 & 82.081 & 265855 & 87.762 & 512 & 98.400 & 2449 \\
\hline 73.433 & 1049 & 82.327 & 1115 & 88.054 & 14122 & 98.434 & 2616 \\
\hline 73.454 & 1310 & 82.377 & 2111 & 88.089 & 518 & 98.471 & 3809 \\
\hline 73.606 & 2385 & 82.408 & 1562 & 88.117 & 432 & 98.564 & 403 \\
\hline 73.649 & 3788 & 82.441 & 2068 & 88.327 & 440 & 98.741 & 2194 \\
\hline 73.915 & 2547 & 82.534 & 550 & 89.055 & 2252 & 98.787 & 2913 \\
\hline 74.038 & 40435 & 82.719 & 1421 & 90.050 & 522 & 99.048 & 26552 \\
\hline 74.072 & 1340 & 82.761 & 1269 & 91.058 & 39597 & 99.083 & 29496 \\
\hline 74.110 & 1238 & 82.919 & 364 & 91.133 & 1179 & 99.120 & 28144 \\
\hline 74.176 & 1648 & 82.977 & 1202 & 91.335 & 392 & 99.161 & 2750 \\
\hline 74.216 & 1585 & 83.053 & 90706 & 92.065 & 10167 & 99.403 & 1791 \\
\hline 74.261 & 796 & 83.089 & 501056 & 93.074 & 55473 & 99.446 & 2098 \\
\hline 74.411 & 360 & 83.165 & 14017 & 93.349 & 405 & 99.714 & 865 \\
\hline 74.462 & 1176 & 83.390 & 3569 & 93.421 & 270 & 99.756 & 1518 \\
\hline 74.500 & 904 & 83.419 & 2878 & 93.460 & 497 & 99.797 & 1362 \\
\hline 74.721 & 545 & 83.448 & 5131 & 94.045 & 2325 & 99.861 & 405 \\
\hline 74.785 & 512 & 83.488 & 3730 & 94.081 & 54434 & 100.056 & 56882 \\
\hline 75.037 & 4694 & 83.518 & 727 & 94.399 & 415 & 100.090 & 2660 \\
\hline 75.075 & 557 & 83.540 & 764 & 94.439 & 473 & 100.124 & 4438 \\
\hline 75.279 & 312 & 83.728 & 3800 & 94.475 & 473 & 100.393 & 779 \\
\hline 76.036 & 935 & 83.767 & 3747 & 94.752 & 479 & 100.430 & 1253 \\
\hline 77.042 & 40514 & 83.992 & 2302 & 94.819 & 434 & 100.723 & 740 \\
\hline 77.323 & 533 & 84.025 & 3512 & 95.053 & 24351 & 100.778 & 676 \\
\hline 78.050 & 14524 & 84.061 & 226540 & 95.089 & 244837 & 101.064 & 95887 \\
\hline 79.058 & 140484 & 84.096 & 171151 & 95.141 & 2666 & 101.101 & 3625 \\
\hline 79.106 & 1241 & 84.141 & 7004 & 95.412 & 1887 & 101.144 & 3237 \\
\hline 79.336 & 2600 & 84.361 & 2878 & 95.442 & 1488 & 101.394 & 868 \\
\hline 79.379 & 968 & 84.397 & 3166 & 95.474 & 2374 & 101.427 & 930 \\
\hline 79.413 & 1616 & 84.427 & 2029 & 95.514 & 1531 & 101.464 & 861 \\
\hline
\end{tabular}


Table S11 (continued). High-resolution mass table for the compound identified as hexadecenoic acid.

\begin{tabular}{|c|c|c|c|c|c|c|c|}
\hline Mass & $\begin{array}{c}\text { Ion } \\
\text { counts }\end{array}$ & Mass & $\begin{array}{c}\text { Ion } \\
\text { counts }\end{array}$ & Mass & $\begin{array}{c}\text { Ion } \\
\text { counts }\end{array}$ & Mass & $\begin{array}{c}\text { Ion } \\
\text { counts }\end{array}$ \\
\hline 101.492 & 660 & 112.056 & 2047 & \begin{tabular}{|l|}
123.050 \\
\end{tabular} & 825 & 131.055 & 902 \\
\hline 101.723 & 636 & 112.092 & 33239 & 123.085 & 89979 & 131.092 & 4826 \\
\hline 101.773 & 743 & 112.128 & 44306 & 123.121 & 29861 & 132.097 & 1571 \\
\hline 101.864 & 410 & 112.185 & 3018 & 123.168 & 1575 & 133.071 & 1030 \\
\hline 102.070 & 16981 & 112.220 & 2866 & 123.188 & 2444 & 133.105 & 25842 \\
\hline 102.110 & 505 & 112.520 & 1619 & 123.228 & 1891 & 133.164 & 291 \\
\hline 102.414 & 559 & 112.588 & 1028 & 123.455 & 467 & 133.196 & 841 \\
\hline 102.445 & 262 & \begin{tabular}{|l|l|}
112.873 \\
\end{tabular} & 1238 & \begin{tabular}{|l|l|}
123.487 \\
\end{tabular} & 479 & 133.587 & 381 \\
\hline 102.476 & 475 & \begin{tabular}{|l|}
112.916 \\
\end{tabular} & 1125 & \begin{tabular}{|l|}
123.519 \\
\end{tabular} & 553 & 134.112 & 15939 \\
\hline 102.734 & 423 & 112.965 & 501 & 123.565 & 727 & 134.211 & 328 \\
\hline 102.785 & 437 & \begin{tabular}{|l|}
113.064 \\
\end{tabular} & 35535 & \begin{tabular}{|l|}
123.857 \\
\end{tabular} & 629 & 135.085 & 3500 \\
\hline 103.059 & 3761 & \begin{tabular}{|l|}
113.099 \\
\end{tabular} & 5453 & 123.906 & 550 & 135.121 & 21557 \\
\hline 104.066 & 2521 & 113.136 & 16690 & 123.937 & 609 & 135.203 & 541 \\
\hline 105.038 & 1603 & \begin{tabular}{|l|}
113.202 \\
\end{tabular} & 2185 & \begin{tabular}{|l|}
124.092 \\
\end{tabular} & 51254 & 136.057 & 957 \\
\hline 105.074 & 17631 & \begin{tabular}{|l|}
113.280 \\
\end{tabular} & 719 & \begin{tabular}{|l|}
124.129 \\
\end{tabular} & 29020 & 136.092 & 3247 \\
\hline 105.130 & 390 & 113.486 & 531 & \begin{tabular}{|l|}
124.187 \\
\end{tabular} & 2662 & 136.128 & 9389 \\
\hline 105.164 & 426 & \begin{tabular}{|l|}
113.532 \\
\end{tabular} & 755 & \begin{tabular}{|l|}
124.237 \\
\end{tabular} & 2125 & 137.063 & 1043 \\
\hline 106.043 & 356 & \begin{tabular}{|l|}
113.566 \\
\end{tabular} & 714 & \begin{tabular}{|l|}
124.294 \\
\end{tabular} & 692 & 137.100 & 51732 \\
\hline 106.081 & 7471 & 113.886 & 706 & 124.460 & 392 & 137.137 & 12843 \\
\hline 107.053 & 2645 & 113.927 & 671 & 124.505 & 409 & 137.201 & 1827 \\
\hline 107.089 & 27611 & \begin{tabular}{|l|}
114.071 \\
\end{tabular} & 83463 & \begin{tabular}{|l|}
124.547 \\
\end{tabular} & 998 & 137.520 & 387 \\
\hline 107.171 & 760 & 114.460 & 301 & 124.620 & 611 & 137.574 & 373 \\
\hline 108.060 & 1912 & 114.501 & 678 & \begin{tabular}{|l|}
124.876 \\
\end{tabular} & 474 & 137.916 & 421 \\
\hline 108.097 & 24289 & \begin{tabular}{|l|}
114.862 \\
\end{tabular} & 615 & \begin{tabular}{|l|}
124.979 \\
\end{tabular} & 659 & 138.108 & 34466 \\
\hline 108.179 & 904 & 114.904 & 512 & 125.064 & 3656 & 138.144 & 20870 \\
\hline 109.069 & 37138 & 115.079 & 72252 & 125.100 & 48860 & 138.205 & 1148 \\
\hline 109.105 & 82642 & 115.118 & 1329 & 125.136 & 27070 & 138.236 & 1321 \\
\hline 109.159 & 2043 & 115.437 & 512 & \begin{tabular}{|l|}
125.544 \\
\end{tabular} & 564 & 138.602 & 458 \\
\hline 109.191 & 3081 & 115.470 & 330 & 125.596 & 644 & 139.080 & 1922 \\
\hline 109.445 & 478 & 115.519 & 936 & 125.937 & 530 & 139.116 & 30503 \\
\hline 109.484 & 940 & 115.569 & 623 & \begin{tabular}{|l|}
126.071 \\
\end{tabular} & 5461 & 139.152 & 8825 \\
\hline 109.526 & 1137 & 115.615 & 414 & 126.106 & 7962 & 140.088 & 3170 \\
\hline 109.825 & 652 & 115.837 & 577 & 126.144 & 20550 & 140.121 & 4677 \\
\hline 109.877 & 820 & 115.887 & 588 & 126.591 & 427 & 140.160 & 8413 \\
\hline 110.077 & 95596 & 116.086 & 16983 & 127.079 & 43445 & 141.095 & 11194 \\
\hline 110.113 & 56645 & 116.564 & 489 & 127.116 & 3282 & 141.132 & 7947 \\
\hline 110.167 & 3954 & 116.860 & 428 & 127.151 & 10875 & 141.167 & 5620 \\
\hline 110.419 & 484 & \begin{tabular}{|l|l|}
117.075 \\
\end{tabular} & 6179 & \begin{tabular}{|l|}
127.228 \\
\end{tabular} & 915 & 141.220 & 497 \\
\hline 110.461 & 1261 & 118.044 & 288 & 128.086 & 13838 & 142.101 & 3756 \\
\hline 110.500 & 1587 & 118.081 & 2657 & \begin{tabular}{|l|}
128.126 \\
\end{tabular} & 321 & 142.136 & 890 \\
\hline 110.541 & 1600 & \begin{tabular}{|l|}
119.057 \\
\end{tabular} & 743 & 128.155 & 1306 & 142.171 & 699 \\
\hline 110.598 & 529 & 119.090 & 26762 & 129.095 & 118805 & 143.111 & 21812 \\
\hline 110.849 & 1514 & 119.178 & 746 & 129.465 & 514 & 143.155 & 469 \\
\hline 110.920 & 564 & 120.061 & 487 & 129.505 & 723 & 143.207 & 630 \\
\hline 111.048 & 1905 & 120.096 & 8806 & 129.545 & 1021 & 144.117 & 5416 \\
\hline 111.084 & 82312 & 120.207 & 428 & \begin{tabular}{|l|l|}
129.609 \\
\end{tabular} & 678 & 145.070 & 553 \\
\hline 111.121 & 98919 & \begin{tabular}{|l|}
121.069 \\
\end{tabular} & 4128 & \begin{tabular}{|l|}
129.872 \\
\end{tabular} & 557 & 145.106 & 2155 \\
\hline 111.180 & 5032 & \begin{tabular}{|l|}
121.105 \\
\end{tabular} & 27425 & \begin{tabular}{|l|}
129.911 \\
\end{tabular} & 682 & 146.070 & 396 \\
\hline 111.472 & 1574 & 122.041 & 734 & \begin{tabular}{|l|}
129.966 \\
\end{tabular} & 594 & 146.111 & 665 \\
\hline 111.503 & 1184 & 122.076 & 2785 & \begin{tabular}{|l|}
130.099 \\
\end{tabular} & 13415 & 147.046 & 478 \\
\hline 111.541 & 2377 & \begin{tabular}{|l|}
122.112 \\
\end{tabular} & 13770 & \begin{tabular}{|l|}
130.177 \\
\end{tabular} & 483 & 147.084 & 1064 \\
\hline 111.857 & 2088 & 122.202 & 535 & 130.264 & 575 & 147.121 & 17748 \\
\hline
\end{tabular}


Table S11 (continued). High-resolution mass table for the compound identified as hexadecenoic acid.

\begin{tabular}{|c|c|c|c|c|c|c|c|}
\hline Mass & $\begin{array}{c}\text { Ion } \\
\text { counts }\end{array}$ & Mass & $\begin{array}{c}\text { Ion } \\
\text { counts }\end{array}$ & Mass & $\begin{array}{c}\text { Ion } \\
\text { counts }\end{array}$ & Mass & $\begin{array}{c}\text { Ion } \\
\text { counts }\end{array}$ \\
\hline 147.203 & 477 & 167.089 & 951 & 190.173 & 1476 & 219.212 & 2938 \\
\hline 147.243 & 427 & 167.147 & 7508 & 191.088 & 512 & 220.150 & 399 \\
\hline 148.128 & 10977 & 167.181 & 2841 & 191.183 & 1169 & 220.222 & 1156 \\
\hline 149.101 & 1692 & 168.117 & 959 & 192.110 & 513 & 221.196 & 1915 \\
\hline 149.136 & 13034 & 168.152 & 1289 & 192.192 & 31014 & 222.200 & 980 \\
\hline 149.214 & 409 & 168.191 & 2629 & 193.163 & 15262 & 223.211 & 556 \\
\hline 150.062 & 535 & 169.126 & 3337 & 193.194 & 5323 & 224.188 & 390 \\
\hline 150.108 & 2195 & 169.163 & 2655 & 193.284 & 578 & 225.190 & 1259 \\
\hline 150.143 & 5445 & 170.132 & 1423 & 194.169 & 6257 & 226.195 & 775 \\
\hline 151.068 & 702 & 171.142 & 30317 & 194.207 & 36291 & 227.205 & 11521 \\
\hline 151.116 & 31301 & 171.249 & 883 & 194.307 & 1508 & 228.210 & 3316 \\
\hline 151.153 & 6725 & 172.061 & 233 & 195.178 & 4741 & 229.220 & 449 \\
\hline 151.218 & 1170 & 172.148 & 6428 & 195.211 & 6849 & 232.942 & 457 \\
\hline 152.066 & 1826 & 173.150 & 1177 & 195.281 & 510 & 234.187 & 485 \\
\hline 152.123 & 18246 & 175.152 & 7051 & 196.149 & 766 & 234.944 & 660 \\
\hline 152.160 & 30744 & 176.070 & 452 & 196.182 & 691 & 235.209 & 956 \\
\hline 153.077 & 1686 & 176.157 & 2457 & 196.221 & 2195 & 236.218 & 73349 \\
\hline 153.131 & 15069 & 177.089 & 411 & 197.157 & 1039 & 236.341 & 2984 \\
\hline 153.166 & 7450 & 177.168 & 4255 & 197.229 & 1473 & 236.730 & 434 \\
\hline 154.079 & 3218 & 178.084 & 865 & 198.164 & 665 & 236.797 & 569 \\
\hline 154.137 & 2748 & 178.173 & 1677 & 199.174 & 19313 & 236.888 & 546 \\
\hline 154.175 & 4739 & 179.090 & 882 & 199.277 & 594 & 237.222 & 14029 \\
\hline 155.110 & 5953 & 179.147 & 12256 & 200.177 & 3263 & 237.336 & 968 \\
\hline 155.147 & 3245 & 179.184 & 1361 & 201.177 & 474 & 237.883 & 420 \\
\hline 155.183 & 2436 & 179.249 & 492 & 203.182 & 462 & 238.224 & 2187 \\
\hline 156.117 & 2046 & 180.086 & 513 & 204.065 & 342 & 238.827 & 421 \\
\hline 156.152 & 594 & 180.154 & 4847 & 204.195 & 407 & 239.239 & 1221 \\
\hline 157.127 & 29664 & 180.191 & 3331 & 205.201 & 861 & 241.219 & 485 \\
\hline 158.133 & 8260 & 181.124 & 732 & 206.208 & 760 & 242.228 & 2669 \\
\hline 159.134 & 1902 & 181.162 & 4741 & 207.039 & 325 & 243.232 & 512 \\
\hline 160.126 & 467 & 182.133 & 652 & 207.179 & 11712 & 249.944 & 1026 \\
\hline 161.137 & 11544 & 182.169 & 722 & 208.185 & 6799 & 250.197 & 492 \\
\hline 162.142 & 4141 & 182.206 & 1950 & 209.190 & 1738 & 251.946 & 1707 \\
\hline 163.078 & 569 & 183.142 & 1439 & 210.167 & 506 & 252.212 & 2694 \\
\hline 163.152 & 6677 & 183.215 & 977 & 211.173 & 3235 & 253.218 & 548 \\
\hline 164.124 & 1218 & 184.149 & 881 & 212.178 & 806 & 254.228 & 6716 \\
\hline 164.158 & 2652 & 185.158 & 33122 & 213.189 & 48114 & 255.231 & 1300 \\
\hline 165.074 & 1368 & 185.264 & 993 & 213.775 & 469 & 256.152 & 496 \\
\hline 165.132 & 18514 & 186.163 & 6568 & 214.193 & 7503 & 256.244 & 18905 \\
\hline 165.168 & 5118 & 187.080 & 470 & 214.292 & 562 & 256.361 & 800 \\
\hline 166.078 & 717 & 187.168 & 1535 & 215.196 & 1217 & 257.247 & 3927 \\
\hline 166.138 & 7280 & 188.166 & 342 & 217.199 & 654 & 257.384 & 529 \\
\hline 166.176 & 11731 & 189.073 & 481 & 218.208 & 8645 & 258.248 & 582 \\
\hline 166.254 & 445 & 189.169 & 3793 & 218.951 & 364 & 263.169 & 442 \\
\hline
\end{tabular}


Table S12. High-resolution mass table for the compound identified as eicosene.

\begin{tabular}{|c|c|c|c|c|c|c|c|c|c|}
\hline Mass & $\begin{array}{c}\text { Ion } \\
\text { counts }\end{array}$ & Mass & $\begin{array}{c}\text { Ion } \\
\text { counts }\end{array}$ & Mass & $\begin{array}{c}\text { Ion } \\
\text { counts }\end{array}$ & Mass & $\begin{array}{c}\text { Ion } \\
\text { counts }\end{array}$ & Mass & $\begin{array}{c}\text { Ion } \\
\text { counts }\end{array}$ \\
\hline 50.019 & 830 & 58.635 & 431 & 81.148 & 474 & 97.792 & 1197 & 119.089 & 847 \\
\hline 51.027 & 2062 & 58.856 & 434 & 82.044 & 456 & 97.839 & 1004 & 120.087 & 387 \\
\hline 52.034 & 1339 & 59.053 & 1269 & 82.081 & 46881 & 97.892 & 367 & 121.071 & 590 \\
\hline 53.042 & 12660 & 60.024 & 11324 & 82.407 & 329 & 98.076 & 6887 & 121.105 & 807 \\
\hline 54.050 & 30483 & 61.031 & 2928 & 83.006 & 436 & 98.111 & 36250 & 122.109 & 445 \\
\hline 54.317 & 329 & 63.027 & 424 & 83.053 & 3584 & 98.171 & 1835 & 123.084 & 1710 \\
\hline 55.021 & 5167 & 65.042 & 3451 & 83.089 & 178110 & 98.436 & 589 & 123.120 & 2108 \\
\hline 55.058 & 212562 & 66.050 & 3386 & 83.138 & 1412 & 98.470 & 524 & 124.092 & 1000 \\
\hline 55.083 & 305 & 67.058 & 44509 & 83.388 & 938 & 98.502 & 666 & 124.128 & 5229 \\
\hline 55.098 & 1867 & 68.028 & 495 & 83.419 & 973 & 98.760 & 509 & 125.065 & 861 \\
\hline 55.301 & 1082 & 68.065 & 34264 & 83.452 & 1595 & 98.800 & 419 & 125.099 & 1125 \\
\hline 55.326 & 1146 & 69.038 & 942 & 83.486 & 1038 & 98.836 & 465 & 125.136 & 24565 \\
\hline 55.347 & 1760 & 69.073 & 161970 & 83.686 & 478 & 99.048 & 853 & 126.088 & 532 \\
\hline 55.375 & 1687 & 69.118 & 1339 & 83.737 & 1082 & 99.081 & 1182 & 126.143 & 11713 \\
\hline 55.425 & 391 & 69.374 & 1225 & 83.766 & 872 & 99.120 & 10407 & 127.077 & 946 \\
\hline 55.576 & 1481 & 69.406 & 1422 & 84.021 & 851 & 99.192 & 457 & 127.150 & 3199 \\
\hline 55.606 & 1581 & 69.676 & 1207 & 84.060 & 5481 & 100.056 & 969 & 128.064 & 621 \\
\hline 55.802 & 816 & 69.724 & 588 & 84.096 & 62189 & 100.126 & 697 & 128.154 & 255 \\
\hline 55.839 & 1120 & 69.921 & 533 & 84.340 & 416 & 101.063 & 2294 & 129.095 & 4013 \\
\hline 56.028 & 1095 & 69.943 & 527 & 84.380 & 544 & 102.069 & 508 & 130.099 & 469 \\
\hline 56.065 & 107257 & 69.969 & 758 & 84.425 & 487 & 103.060 & 419 & 131.090 & 415 \\
\hline 56.269 & 353 & 70.044 & 1654 & 84.460 & 393 & 104.064 & 435 & 133.104 & 596 \\
\hline 56.313 & 1089 & 70.081 & 100729 & 85.032 & 627 & 105.036 & 409 & 134.111 & 354 \\
\hline 56.335 & 936 & 70.355 & 357 & 85.067 & 1244 & 105.073 & 1534 & 135.082 & 413 \\
\hline 56.358 & 1246 & 70.384 & 374 & 85.104 & 55883 & 106.079 & 596 & 135.121 & 731 \\
\hline 56.381 & 871 & 70.451 & 923 & 85.407 & 384 & 107.054 & 1176 & 136.130 & 373 \\
\hline 56.528 & 295 & 70.661 & 537 & 85.436 & 234 & 107.089 & 1335 & 137.049 & 362 \\
\hline 56.583 & 1106 & 70.730 & 566 & 85.466 & 432 & 108.069 & 317 & 137.101 & 801 \\
\hline 56.615 & 817 & 70.975 & 468 & 86.067 & 277 & 108.097 & 755 & 137.135 & 1093 \\
\hline 56.818 & 732 & 71.015 & 336 & 86.108 & 4296 & 109.067 & 945 & 138.109 & 670 \\
\hline 56.856 & 626 & 71.052 & 2058 & 87.048 & 2699 & 109.105 & 5253 & 138.144 & 3127 \\
\hline 56.882 & 426 & 71.089 & 99827 & 87.085 & 382 & 110.076 & 1647 & 139.115 & 705 \\
\hline 57.037 & 2570 & 71.393 & 643 & 88.056 & 435 & 110.112 & 9719 & 139.151 & 8028 \\
\hline 57.073 & 208768 & 71.429 & 534 & 89.046 & 361 & 111.083 & 2389 & 140.159 & 7180 \\
\hline 57.320 & 1559 & 71.466 & 480 & 91.058 & 3802 & 111.120 & 63634 & 141.080 & 481 \\
\hline 57.346 & 1424 & 71.504 & 456 & 92.064 & 1175 & 111.462 & 413 & 141.165 & 1805 \\
\hline 57.370 & 2181 & 71.668 & 413 & 93.074 & 3017 & 111.510 & 576 & 143.109 & 1161 \\
\hline 57.447 & 324 & 71.728 & 441 & 94.047 & 419 & 111.564 & 497 & 147.123 & 348 \\
\hline 57.589 & 1631 & 72.003 & 360 & 94.081 & 2179 & 111.878 & 516 & 149.133 & 312 \\
\hline 57.622 & 1777 & 72.026 & 209 & 95.053 & 1136 & 112.092 & 1595 & 151.120 & 315 \\
\hline 57.847 & 1348 & 72.092 & 5359 & 95.089 & 13749 & 112.127 & 20605 & 151.152 & 397 \\
\hline 57.888 & 869 & 73.032 & 13280 & 95.145 & 298 & 112.211 & 992 & 152.059 & 424 \\
\hline 57.928 & 376 & 73.068 & 869 & 96.061 & 2249 & 112.826 & 344 & 152.160 & 2156 \\
\hline 58.045 & 1206 & 74.038 & 1395 & 96.097 & 23858 & 113.063 & 859 & 153.070 & 387 \\
\hline 58.077 & 9510 & 75.030 & 412 & 96.168 & 527 & 113.135 & 5142 & 153.167 & 3781 \\
\hline 58.113 & 953 & 76.034 & 269 & 97.035 & 443 & 113.216 & 343 & 154.079 & 1343 \\
\hline 58.146 & 687 & 77.042 & 3940 & 97.068 & 4055 & 114.071 & 1204 & 154.174 & 4741 \\
\hline 58.320 & 455 & 78.050 & 1929 & 97.105 & 146722 & 114.137 & 430 & 155.084 & 412 \\
\hline 58.352 & 613 & 79.058 & 8275 & 97.428 & 943 & 115.079 & 2379 & 155.183 & 994 \\
\hline 58.382 & 529 & 80.065 & 4107 & 97.460 & 959 & 116.077 & 718 & 157.126 & 994 \\
\hline 58.575 & 484 & 81.037 & 373 & 97.496 & 1505 & 117.073 & 808 & 159.115 & 296 \\
\hline 58.604 & 471 & 81.073 & 26016 & 97.534 & 1027 & 118.078 & 337 & 161.134 & 191 \\
\hline
\end{tabular}


Table S12 (continued). High-resolution mass table for the compound identified as eicosene.

\begin{tabular}{|c|c|c|c|c|c|c|c|c|c|}
\hline Mass & $\begin{array}{c}\text { Ion } \\
\text { counts }\end{array}$ & Mass & $\begin{array}{c}\text { Ion } \\
\text { counts }\end{array}$ & Mass & $\begin{array}{c}\text { Ion } \\
\text { counts }\end{array}$ & Mass & $\begin{array}{c}\text { Ion } \\
\text { counts }\end{array}$ & Mass & $\begin{array}{c}\text { Ion } \\
\text { counts }\end{array}$ \\
\hline 166.175 & 1490 & 181.199 & 1152 & 195.212 & 923 & 213.187 & 978 & 249.942 & 376 \\
\hline 167.183 & 2210 & 182.207 & 1850 & 196.221 & 1627 & 222.234 & 479 & 251.948 & 557 \\
\hline 168.190 & 3166 & 183.212 & 490 & 199.173 & 1403 & 224.255 & 592 & 252.284 & 1296 \\
\hline 169.194 & 760 & 185.158 & 1272 & 208.226 & 529 & 234.942 & 277 & 253.290 & 273 \\
\hline 171.141 & 809 & 192.190 & 480 & 209.227 & 415 & 236.216 & 1058 & 280.316 & 778 \\
\hline 180.189 & 998 & 194.207 & 972 & 210.237 & 1295 & 242.223 & 347 & &
\end{tabular}


Table S13. High-resolution mass table for the compound identified as heptadecenoic acid.

\begin{tabular}{|c|c|c|c|c|c|c|c|}
\hline Mass & $\begin{array}{c}\text { Ion } \\
\text { counts }\end{array}$ & Mass & $\begin{array}{c}\text { Ion } \\
\text { counts }\end{array}$ & Mass & $\begin{array}{c}\text { Ion } \\
\text { counts }\end{array}$ & Mass & $\begin{array}{c}\text { Ion } \\
\text { counts }\end{array}$ \\
\hline 50.019 & 829 & 69.073 & 91518 & 86.108 & 1430 & 111.084 & 10108 \\
\hline 51.026 & 1714 & 69.374 & 576 & 87.048 & 13543 & 111.120 & 13724 \\
\hline 52.034 & 1423 & 69.396 & 697 & 87.083 & 1010 & 111.184 & 473 \\
\hline 53.042 & 13273 & 69.673 & 540 & 88.053 & 1701 & 111.217 & 420 \\
\hline 54.050 & 29774 & 69.722 & 384 & 89.054 & 489 & 112.091 & 5206 \\
\hline 54.108 & 647 & 70.045 & 2675 & 91.058 & 6334 & 112.127 & 5537 \\
\hline 55.021 & 13114 & 70.081 & 40845 & 92.064 & 1840 & 113.063 & 4018 \\
\hline 55.057 & 131080 & 70.452 & 350 & 93.073 & 7597 & 113.098 & 882 \\
\hline 55.301 & 662 & 71.052 & 8256 & 94.045 & 385 & 113.135 & 2312 \\
\hline 55.326 & 769 & 71.089 & 33172 & 94.081 & 6328 & 114.071 & 8534 \\
\hline 55.357 & 1167 & 71.154 & 900 & 95.052 & 3232 & 114.140 & 379 \\
\hline 55.386 & 721 & 71.671 & 322 & 95.089 & 27495 & 115.057 & 935 \\
\hline 55.593 & 1007 & 72.023 & 397 & 95.166 & 991 & 115.078 & 8080 \\
\hline 55.783 & 442 & 72.058 & 1025 & 96.060 & 13282 & 115.124 & 259 \\
\hline 55.818 & 580 & 72.092 & 2003 & 96.096 & 17214 & 116.085 & 2310 \\
\hline 55.853 & 534 & 73.032 & 54014 & 96.143 & 358 & 117.075 & 1315 \\
\hline 56.027 & 1962 & 73.068 & 4303 & 96.164 & 589 & 118.080 & 389 \\
\hline 56.065 & 46324 & 73.331 & 423 & 96.407 & 361 & 119.089 & 3554 \\
\hline 56.128 & 1387 & 73.367 & 417 & 97.031 & 517 & 120.095 & 1493 \\
\hline 56.285 & 394 & 73.397 & 375 & 97.068 & 16564 & 121.070 & 585 \\
\hline 56.322 & 594 & 74.039 & 7869 & 97.104 & 39827 & 121.104 & 3988 \\
\hline 56.360 & 437 & 75.037 & 889 & 97.186 & 1493 & 122.076 & 438 \\
\hline 56.594 & 445 & 76.034 & 332 & 97.433 & 476 & 122.111 & 1758 \\
\hline 57.037 & 7325 & 77.042 & 5996 & 97.492 & 478 & 123.084 & 8496 \\
\hline 57.073 & 77917 & 78.049 & 2649 & 97.798 & 379 & 123.120 & 4179 \\
\hline 57.326 & 525 & 79.058 & 17106 & 97.827 & 306 & 123.198 & 417 \\
\hline 57.347 & 375 & 79.138 & 413 & 98.076 & 22536 & 124.091 & 5044 \\
\hline 57.368 & 554 & 80.065 & 9495 & 98.111 & 10774 & 124.128 & 3347 \\
\hline 57.393 & 496 & 81.036 & 1385 & 98.153 & 999 & 125.063 & 540 \\
\hline 57.562 & 366 & 81.073 & 44168 & 99.047 & 3227 & 125.099 & 5857 \\
\hline 57.586 & 447 & 81.368 & 386 & 99.082 & 3776 & 125.136 & 4043 \\
\hline 57.623 & 468 & 81.397 & 221 & 99.119 & 4160 & 126.070 & 771 \\
\hline 57.835 & 440 & 81.439 & 448 & 100.055 & 5731 & 126.105 & 1097 \\
\hline 58.044 & 2356 & 82.045 & 2174 & 100.097 & 391 & 126.143 & 2725 \\
\hline 58.077 & 3646 & 82.081 & 29834 & 100.124 & 662 & 127.079 & 4873 \\
\hline 59.053 & 5178 & 82.153 & 654 & 101.063 & 11287 & 127.150 & 1559 \\
\hline 59.085 & 279 & 83.053 & 11732 & 101.101 & 332 & 128.085 & 2061 \\
\hline 60.024 & 41731 & 83.089 & 62537 & 102.069 & 1930 & 128.153 & 254 \\
\hline 60.058 & 686 & 83.160 & 1883 & 103.060 & 552 & 129.094 & 13802 \\
\hline 60.304 & 408 & 83.419 & 533 & 104.066 & 448 & 129.181 & 457 \\
\hline 61.031 & 9321 & 83.475 & 490 & 105.039 & 503 & 130.099 & 2158 \\
\hline 61.099 & 408 & 83.761 & 462 & 105.073 & 2858 & 131.089 & 1057 \\
\hline 62.027 & 458 & 84.060 & 23603 & 106.080 & 1442 & 133.104 & 3042 \\
\hline 63.026 & 633 & 84.096 & 20599 & 107.052 & 716 & 134.111 & 1948 \\
\hline 65.042 & 4690 & 84.144 & 671 & 107.088 & 4117 & 135.084 & 719 \\
\hline 66.050 & 3757 & 84.427 & 300 & 108.059 & 491 & 135.120 & 3262 \\
\hline 67.058 & 48301 & 85.032 & 1425 & 108.096 & 2926 & 136.091 & 577 \\
\hline 67.354 & 448 & 85.067 & 5996 & 109.068 & 4580 & 136.126 & 1267 \\
\hline 67.404 & 371 & 85.104 & 18273 & 109.104 & 10510 & 137.099 & 5629 \\
\hline 68.029 & 719 & 85.179 & 464 & 110.076 & 9851 & 137.136 & 2111 \\
\hline 68.065 & 32927 & 86.040 & 1131 & 110.112 & 6976 & 138.107 & 3709 \\
\hline 69.036 & 2254 & 86.073 & 729 & 110.165 & 377 & 138.143 & 2274 \\
\hline
\end{tabular}


Table S13 (continued). High-resolution mass table for the compound identified as heptadecenoic acid.

\begin{tabular}{|c|c|c|c|c|c|c|c|}
\hline Mass & $\begin{array}{c}\text { Ion } \\
\text { counts }\end{array}$ & Mass & $\begin{array}{c}\text { Ion } \\
\text { counts }\end{array}$ & Mass & $\begin{array}{c}\text { Ion } \\
\text { counts }\end{array}$ & Mass & $\begin{array}{c}\text { Ion } \\
\text { counts }\end{array}$ \\
\hline 139.115 & 4190 & 155.145 & 659 & 179.147 & 1402 & 211.246 & 380 \\
\hline 139.150 & 1137 & 155.180 & 649 & 180.153 & 639 & 213.187 & 5331 \\
\hline 140.089 & 409 & 156.117 & 431 & 180.192 & 788 & 214.192 & 1017 \\
\hline 140.120 & 646 & 157.126 & 2805 & 181.161 & 831 & 217.158 & 436 \\
\hline 140.159 & 1457 & 158.130 & 908 & 185.156 & 4283 & 221.193 & 1395 \\
\hline 141.094 & 1860 & 159.101 & 448 & 186.163 & 1274 & 222.198 & 515 \\
\hline 141.166 & 1146 & 161.136 & 1589 & 186.958 & 318 & 223.206 & 601 \\
\hline 142.095 & 529 & 162.140 & 553 & 187.173 & 343 & 227.203 & 3299 \\
\hline 143.110 & 3384 & 163.151 & 1319 & 189.167 & 567 & 228.208 & 578 \\
\hline 144.116 & 660 & 164.158 & 565 & 193.162 & 1075 & 232.223 & 803 \\
\hline 145.104 & 366 & 165.073 & 440 & 194.167 & 643 & 233.221 & 388 \\
\hline 147.082 & 482 & 165.131 & 2140 & 194.208 & 567 & 234.944 & 359 \\
\hline 147.120 & 2307 & 165.168 & 414 & 195.177 & 997 & 236.214 & 311 \\
\hline 148.128 & 1280 & 166.138 & 877 & 196.206 & 388 & 241.219 & 1034 \\
\hline 149.136 & 2223 & 166.174 & 2447 & 199.171 & 1673 & 249.943 & 407 \\
\hline 150.143 & 996 & 167.146 & 1647 & 200.177 & 362 & 250.231 & 6676 \\
\hline 151.115 & 3556 & 168.191 & 440 & 202.077 & 443 & 251.236 & 1577 \\
\hline 151.151 & 711 & 169.122 & 379 & 203.170 & 397 & 251.946 & 593 \\
\hline 152.121 & 2056 & 169.166 & 410 & 205.200 & 447 & 256.245 & 1104 \\
\hline 152.159 & 2234 & 171.141 & 3858 & 206.205 & 2596 & 263.166 & 1671 \\
\hline 153.129 & 2355 & 172.147 & 916 & 207.177 & 1633 & 268.240 & 503 \\
\hline 153.164 & 706 & 175.150 & 956 & 208.181 & 631 & 270.258 & 1183 \\
\hline 154.173 & 815 & 177.166 & 1078 & 208.221 & 2892 & & \\
\hline 155.109 & 939 & 178.163 & 331 & 209.224 & 732 & & \\
\hline
\end{tabular}


Table S14. High-resolution mass table for the compound identified as octadecenoic acid.

\begin{tabular}{|c|c|c|c|c|c|c|c|c|c|}
\hline Mass & $\begin{array}{c}\text { Ion } \\
\text { counts }\end{array}$ & Mass & $\begin{array}{c}\text { Ion } \\
\text { counts }\end{array}$ & Mass & $\begin{array}{c}\text { Ion } \\
\text { counts }\end{array}$ & Mass & $\begin{array}{c}\text { Ion } \\
\text { counts }\end{array}$ & Mass & $\begin{array}{c}\text { Ion } \\
\text { counts }\end{array}$ \\
\hline 49.011 & 314 & 56.092 & 901 & 60.404 & 331 & 68.999 & 1286 & 73.139 & 1275 \\
\hline 50.019 & 2521 & 56.104 & 3024 & 60.562 & 963 & 69.037 & 9976 & 73.313 & 966 \\
\hline 51.026 & 7491 & 56.275 & 1791 & 60.600 & 922 & 69.073 & 516685 & 73.341 & 868 \\
\hline 52.034 & 5542 & 56.313 & 3457 & 60.656 & 368 & 69.105 & 614 & 73.365 & 1370 \\
\hline 53.006 & 698 & 56.335 & 2041 & 60.791 & 439 & 69.117 & 4557 & 73.398 & 1212 \\
\hline 53.042 & 68648 & 56.351 & 2106 & 60.823 & 554 & 69.342 & 1644 & 73.452 & 354 \\
\hline 53.306 & 596 & 56.369 & 2856 & 60.851 & 521 & 69.353 & 1265 & 73.633 & 1066 \\
\hline 53.326 & 677 & 56.435 & 194 & 61.031 & 20306 & 69.374 & 2360 & 73.681 & 919 \\
\hline 53.399 & 278 & 56.555 & 2426 & 61.099 & 780 & 69.408 & 4560 & 73.705 & 577 \\
\hline 53.554 & 435 & 56.583 & 2896 & 61.316 & 284 & 69.465 & 1103 & 73.874 & 414 \\
\hline 53.583 & 416 & 56.613 & 2312 & 61.358 & 465 & 69.481 & 1464 & 73.904 & 493 \\
\hline 53.801 & 397 & 56.820 & 2374 & 61.575 & 400 & 69.656 & 3454 & 73.925 & 385 \\
\hline 54.014 & 760 & 56.850 & 1998 & 62.026 & 1596 & 69.685 & 2825 & 73.946 & 611 \\
\hline 54.050 & 213602 & 57.037 & 44924 & 63.026 & 1993 & 69.706 & 1828 & 74.039 & 17654 \\
\hline 54.089 & 1879 & 57.073 & 294906 & 64.037 & 1044 & 69.724 & 1758 & 74.072 & 979 \\
\hline 54.104 & 3036 & 57.099 & 1090 & 65.042 & 28789 & 69.743 & 1370 & 74.106 & 371 \\
\hline 54.114 & 3892 & 57.112 & 3083 & 65.105 & 607 & 69.908 & 1897 & 74.148 & 475 \\
\hline 54.149 & 2590 & 57.279 & 767 & 65.137 & 407 & 69.931 & 2206 & 74.184 & 490 \\
\hline 54.291 & 1319 & 57.320 & 3006 & 65.330 & 405 & 69.961 & 2485 & 74.226 & 476 \\
\hline 54.315 & 1242 & 57.346 & 2173 & 66.050 & 24090 & 69.996 & 1787 & 74.469 & 422 \\
\hline 54.343 & 2053 & 57.369 & 3356 & 66.125 & 557 & 70.044 & 8758 & 75.037 & 2833 \\
\hline 54.414 & 293 & 57.392 & 1218 & 66.871 & 446 & 70.081 & 210498 & 76.035 & 840 \\
\hline 54.575 & 1512 & 57.404 & 1826 & 66.931 & 448 & 70.126 & 2021 & 77.042 & 39559 \\
\hline 54.622 & 756 & 57.446 & 351 & 67.019 & 753 & 70.352 & 380 & 77.116 & 1117 \\
\hline 54.779 & 815 & 57.561 & 1826 & 67.058 & 386706 & 70.383 & 890 & 77.153 & 420 \\
\hline 54.806 & 1290 & 57.589 & 2454 & 67.101 & 2931 & 70.412 & 1432 & 77.319 & 379 \\
\hline 54.830 & 1165 & 57.617 & 2650 & 67.329 & 2410 & 70.458 & 2137 & 77.359 & 227 \\
\hline 54.859 & 820 & 57.782 & 565 & 67.353 & 2072 & 70.494 & 1102 & 77.552 & 264 \\
\hline 54.902 & 412 & 57.812 & 1165 & 67.382 & 3485 & 70.548 & 262 & 77.661 & 361 \\
\hline 54.937 & 348 & 57.832 & 1248 & 67.415 & 2516 & 70.671 & 961 & 78.049 & 14451 \\
\hline 55.021 & 57247 & 57.856 & 1927 & 67.442 & 758 & 70.701 & 1610 & 79.058 & 126531 \\
\hline 55.058 & 610041 & 58.044 & 12880 & 67.461 & 951 & 70.929 & 394 & 79.338 & 1883 \\
\hline 55.095 & 28048 & 58.077 & 13687 & 67.611 & 2146 & 70.968 & 653 & 79.378 & 955 \\
\hline 55.272 & 3299 & 58.111 & 1724 & 67.631 & 2142 & 71.017 & 1343 & 79.428 & 1267 \\
\hline 55.309 & 3444 & 58.287 & 534 & 67.657 & 2579 & 71.052 & 45087 & 79.498 & 430 \\
\hline 55.326 & 4650 & 58.346 & 1188 & 67.688 & 2567 & 71.089 & 118943 & 79.622 & 402 \\
\hline 55.359 & 6815 & 58.382 & 731 & 67.885 & 2015 & 71.134 & 1849 & 79.664 & 757 \\
\hline 55.387 & 3107 & 58.549 & 420 & 67.915 & 1804 & 71.152 & 1605 & 79.688 & 524 \\
\hline 55.408 & 1808 & 58.575 & 716 & 67.944 & 1925 & 71.362 & 461 & 79.717 & 725 \\
\hline 55.423 & 660 & 58.600 & 857 & 67.997 & 665 & 71.393 & 723 & 79.937 & 432 \\
\hline 55.430 & 539 & 58.841 & 590 & 68.029 & 2740 & 71.440 & 692 & 79.980 & 544 \\
\hline 55.527 & 1477 & 59.053 & 25320 & 68.065 & 223523 & 71.480 & 566 & 80.065 & 83992 \\
\hline 55.538 & 1263 & 59.086 & 888 & 68.111 & 2438 & 71.504 & 304 & 80.144 & 1161 \\
\hline 55.552 & 2604 & 59.323 & 422 & 68.340 & 802 & 71.635 & 404 & 80.347 & 431 \\
\hline 55.578 & 5664 & 59.354 & 436 & 68.363 & 1224 & 71.677 & 509 & 80.390 & 519 \\
\hline 55.618 & 4640 & 59.814 & 371 & 68.401 & 2440 & 71.703 & 358 & 80.436 & 497 \\
\hline 55.785 & 2787 & 60.024 & 114876 & 68.433 & 2352 & 71.729 & 399 & 80.653 & 315 \\
\hline 55.815 & 4334 & 60.088 & 3215 & 68.474 & 1111 & 72.024 & 558 & 80.693 & 431 \\
\hline 55.842 & 3318 & 60.284 & 618 & 68.652 & 1915 & 72.058 & 4752 & 81.037 & 5617 \\
\hline 55.866 & 3158 & 60.305 & 731 & 68.684 & 2146 & 72.092 & 6278 & 81.073 & 317731 \\
\hline 56.028 & 8024 & 60.338 & 1397 & 68.914 & 1503 & 73.032 & 147580 & 81.121 & 2358 \\
\hline 56.065 & 230124 & 60.387 & 470 & 68.953 & 1721 & 73.068 & 21438 & 81.371 & 1981 \\
\hline
\end{tabular}


Table S14 (continued). High-resolution mass table for the compound identified as octadecenoic acid.

\begin{tabular}{|c|c|c|c|c|c|c|c|c|c|}
\hline Mass & $\begin{array}{c}\text { Ion } \\
\text { counts }\end{array}$ & Mass & $\begin{array}{c}\text { Ion } \\
\text { counts }\end{array}$ & Mass & $\begin{array}{c}\text { Ion } \\
\text { counts }\end{array}$ & Mass & $\begin{array}{c}\text { Ion } \\
\text { counts }\end{array}$ & Mass & $\begin{array}{c}\text { Ion } \\
\text { counts }\end{array}$ \\
\hline 81.398 & 1744 & 86.040 & 4077 & 97.424 & 2524 & \begin{tabular}{|l|l|}
107.166 \\
\end{tabular} & 1275 & 113.266 & 651 \\
\hline 81.432 & 2963 & 86.073 & 3353 & 97.437 & 1415 & \begin{tabular}{|l|}
107.458 \\
\end{tabular} & 395 & 113.510 & 519 \\
\hline 81.476 & 2242 & 86.108 & 3893 & 97.461 & 2978 & \begin{tabular}{|l|l|}
107.493 \\
\end{tabular} & 399 & 113.566 & 509 \\
\hline 81.521 & 781 & 87.048 & 46925 & 97.500 & 2695 & 107.545 & 393 & 113.863 & 398 \\
\hline 81.707 & 2416 & 87.084 & 5355 & 97.531 & 1817 & 107.826 & 366 & 113.899 & 402 \\
\hline 81.739 & 1895 & 87.382 & 362 & 97.760 & 2124 & \begin{tabular}{|l|l|}
108.060 \\
\end{tabular} & 2196 & 114.071 & 50543 \\
\hline 81.771 & 1676 & 87.404 & 296 & 97.786 & 1265 & 108.096 & 30465 & 114.523 & 475 \\
\hline 81.984 & 1766 & 87.442 & 475 & 97.803 & 995 & 108.788 & 332 & 114.876 & 493 \\
\hline 82.045 & 10283 & 88.054 & 5902 & 97.820 & 1216 & 109.068 & 25410 & 115.078 & 31229 \\
\hline 82.081 & 220283 & 88.089 & 447 & 97.841 & 1656 & 109.105 & 91891 & 115.118 & 1140 \\
\hline 82.374 & 1579 & 89.043 & 1490 & 98.041 & 1349 & \begin{tabular}{|l|l|}
109.158 \\
\end{tabular} & 1389 & 115.168 & 882 \\
\hline 82.408 & 1343 & 90.049 & 365 & 98.076 & 102062 & 109.444 & 553 & 115.468 & 338 \\
\hline 82.435 & 1753 & 91.058 & 44158 & 98.111 & 61575 & 109.485 & 1073 & 115.534 & 479 \\
\hline 82.529 & 441 & 91.434 & 430 & 98.162 & 5247 & 109.524 & 1050 & 116.085 & 7186 \\
\hline 82.670 & 624 & 91.573 & 481 & 98.406 & 1698 & 109.843 & 733 & 116.143 & 266 \\
\hline 82.742 & 927 & 92.064 & 10762 & 98.434 & 2201 & 109.879 & 372 & 116.175 & 462 \\
\hline 82.793 & 405 & 93.073 & 61716 & 98.468 & 1262 & 109.902 & 519 & 117.073 & 6676 \\
\hline 82.980 & 829 & 93.386 & 461 & 98.493 & 2067 & 110.076 & 55436 & 118.074 & 4736 \\
\hline 83.053 & 51401 & 93.423 & 550 & 98.778 & 2065 & 110.112 & 55790 & 119.054 & 708 \\
\hline 83.089 & 363167 & 93.758 & 459 & 99.048 & 13693 & 110.164 & 2283 & 119.089 & 20539 \\
\hline 83.164 & 10185 & 94.045 & 1752 & 99.082 & 16918 & 110.185 & 1154 & 119.184 & 498 \\
\hline 83.380 & 1645 & 94.081 & 69845 & 99.119 & 12262 & 110.205 & 2721 & 120.060 & 631 \\
\hline 83.395 & 1370 & 94.167 & 1338 & 99.166 & 1677 & 110.458 & 942 & 120.095 & 7972 \\
\hline 83.418 & 1938 & 94.395 & 426 & 99.437 & 1307 & \begin{tabular}{|l|}
110.499 \\
\end{tabular} & 1355 & 121.068 & 6335 \\
\hline 83.453 & 3664 & 94.433 & 406 & 99.719 & 770 & 110.558 & 1117 & 121.104 & 28638 \\
\hline 83.491 & 2066 & 94.456 & 595 & 99.762 & 547 & \begin{tabular}{|l|}
110.795 \\
\end{tabular} & 448 & 121.186 & 939 \\
\hline 83.518 & 575 & 94.805 & 434 & 99.801 & 567 & 110.859 & 1085 & 122.038 & 503 \\
\hline 83.538 & 794 & 94.991 & 331 & 100.055 & 31840 & 111.048 & 1771 & 122.076 & 6159 \\
\hline 83.701 & 2058 & 95.053 & 13766 & 100.090 & 1941 & \begin{tabular}{|l|}
111.084 \\
\end{tabular} & 52161 & 122.112 & 16331 \\
\hline 83.733 & 2364 & 95.089 & 203332 & 100.123 & 2212 & \begin{tabular}{|l|}
11.120 \\
\end{tabular} & 82310 & 122.189 & 473 \\
\hline 83.767 & 2477 & 95.140 & 1709 & 100.378 & 504 & \begin{tabular}{|l|}
111.178 \\
\end{tabular} & 3116 & 123.084 & 48508 \\
\hline 84.026 & 2407 & 95.409 & 1430 & 100.420 & 612 & \begin{tabular}{|l|}
111.208 \\
\end{tabular} & 3922 & 123.120 & 33011 \\
\hline 84.060 & 114860 & 95.441 & 1559 & 100.453 & 372 & \begin{tabular}{|l|}
111.427 \\
\end{tabular} & 294 & 123.216 & 1812 \\
\hline 84.096 & 113336 & 95.480 & 1911 & 100.483 & 468 & \begin{tabular}{|l|}
11.468 \\
\end{tabular} & 1006 & 123.490 & 522 \\
\hline 84.142 & 4374 & 95.572 & 242 & 100.758 & 558 & \begin{tabular}{|l|}
111.501 \\
\end{tabular} & 1446 & 123.530 & 587 \\
\hline 84.340 & 1150 & 95.757 & 1459 & 101.063 & 50635 & \begin{tabular}{|l|}
111.551 \\
\end{tabular} & 1637 & 123.588 & 494 \\
\hline 84.364 & 1322 & 95.795 & 1461 & 101.100 & 2321 & 111.884 & 1359 & 123.866 & 417 \\
\hline 84.392 & 1956 & 95.834 & 830 & 101.398 & 433 & 112.055 & 2363 & 123.910 & 569 \\
\hline 84.427 & 2062 & 96.060 & 75590 & 101.429 & 427 & \begin{tabular}{|l|}
112.091 \\
\end{tabular} & 23669 & 124.091 & 32402 \\
\hline 84.564 & 1558 & 96.096 & 130912 & 101.458 & 582 & 112.127 & 31181 & 124.128 & 27530 \\
\hline 84.633 & 453 & 96.132 & 867 & 101.761 & 465 & \begin{tabular}{|l|}
112.173 \\
\end{tabular} & 1251 & 124.183 & 1261 \\
\hline 84.661 & 799 & 96.175 & 5249 & 102.069 & 7623 & \begin{tabular}{|l|}
112.208 \\
\end{tabular} & 2596 & 124.219 & 1954 \\
\hline 84.719 & 1394 & 96.416 & 2289 & 102.115 & 513 & \begin{tabular}{|l|}
112.278 \\
\end{tabular} & 777 & 124.493 & 387 \\
\hline 84.776 & 485 & 96.452 & 1516 & 102.157 & 397 & 112.478 & 484 & 124.532 & 458 \\
\hline 84.950 & 460 & 96.482 & 2251 & 103.059 & 3165 & \begin{tabular}{|l|}
112.512 \\
\end{tabular} & 605 & 124.573 & 620 \\
\hline 84.983 & 404 & 96.552 & 538 & 104.065 & 2820 & \begin{tabular}{|l|}
112.540 \\
\end{tabular} & 1056 & 124.650 & 383 \\
\hline 85.032 & 6276 & 96.746 & 1835 & 105.037 & 1164 & 112.582 & 674 & 124.903 & 471 \\
\hline 85.068 & 33418 & 96.781 & 1992 & 105.073 & 19832 & 112.888 & 807 & 124.962 & 475 \\
\hline 85.104 & 59112 & 97.032 & 2948 & 105.155 & 457 & 113.063 & 22211 & 125.008 & 329 \\
\hline 85.407 & 526 & 97.068 & 81319 & 106.080 & 8468 & 113.098 & 4342 & 125.063 & 1767 \\
\hline 85.438 & 491 & 97.104 & 234792 & 107.053 & 2920 & \begin{tabular}{|l|}
113.134 \\
\end{tabular} & 6859 & 125.099 & 32771 \\
\hline 85.493 & 357 & 97.182 & 8423 & 107.089 & 37687 & 113.196 & 1337 & 125.136 & 25034 \\
\hline
\end{tabular}


Table S14 (continued). High-resolution mass table for the compound identified as octadecenoic acid.

\begin{tabular}{|c|c|c|c|c|c|c|c|c|c|}
\hline Mass & $\begin{array}{c}\text { Ion } \\
\text { counts }\end{array}$ & Mass & $\begin{array}{c}\text { Ion } \\
\text { counts }\end{array}$ & Mass & $\begin{array}{c}\text { Ion } \\
\text { counts }\end{array}$ & Mass & $\begin{array}{c}\text { Ion } \\
\text { counts }\end{array}$ & Mass & $\begin{array}{c}\text { Ion } \\
\text { counts }\end{array}$ \\
\hline 125.237 & 1550 & 141.166 & 2761 & 166.175 & 8059 & \begin{tabular}{|l|l|}
194.169 \\
\end{tabular} & 3888 & 234.224 & 468 \\
\hline 125.569 & 558 & 142.101 & 3167 & 166.242 & 461 & \begin{tabular}{|l|l|}
194.206 \\
\end{tabular} & 3486 & 234.941 & 533 \\
\hline 126.071 & 9112 & 143.110 & 9545 & 167.110 & 3041 & \begin{tabular}{|l|}
195.142 \\
\end{tabular} & 2018 & 235.209 & 6583 \\
\hline 126.106 & 5973 & 144.115 & 2219 & 167.146 & 6389 & \begin{tabular}{|l|l|}
195.177 \\
\end{tabular} & 3809 & 236.215 & 4625 \\
\hline 126.143 & 15818 & 145.070 & 701 & 167.180 & 2344 & \begin{tabular}{|l|}
196.148 \\
\end{tabular} & 2741 & 237.219 & 1416 \\
\hline 126.217 & 517 & 145.105 & 2896 & 168.118 & 3761 & \begin{tabular}{|l|l|}
197.156 \\
\end{tabular} & 1111 & 238.158 & 765 \\
\hline 126.249 & 560 & 146.110 & 1299 & 168.153 & 1432 & \begin{tabular}{|l|l|}
198.162 \\
\end{tabular} & 597 & 239.204 & 1834 \\
\hline 126.307 & 450 & 147.084 & 2380 & 168.190 & 1910 & \begin{tabular}{|l|l|}
199.173 \\
\end{tabular} & 4767 & 240.205 & 385 \\
\hline 127.079 & 28584 & 147.121 & 13795 & 169.125 & 3225 & 200.179 & 1482 & 241.219 & 2272 \\
\hline 127.116 & 1363 & 147.209 & 444 & 169.162 & 3545 & \begin{tabular}{|l|l|}
201.177 \\
\end{tabular} & 617 & 242.226 & 802 \\
\hline 127.151 & 4183 & 148.089 & 717 & 170.131 & 1084 & 202.081 & 337 & 243.233 & 444 \\
\hline 127.491 & 362 & 148.127 & 7918 & 171.141 & 7016 & 203.095 & 457 & 246.168 & 353 \\
\hline 128.085 & 10355 & 149.100 & 7100 & 172.147 & 1742 & 203.183 & 2101 & 246.237 & 4122 \\
\hline 128.158 & 601 & 149.136 & 12963 & 173.100 & 529 & 204.187 & 907 & 247.244 & 1170 \\
\hline 129.094 & 32449 & 149.233 & 476 & 173.136 & 2130 & 205.198 & 1423 & 248.181 & 766 \\
\hline 129.186 & 924 & 150.107 & 6924 & 174.141 & 491 & 206.205 & 1251 & 249.225 & 1201 \\
\hline 130.078 & 1132 & 150.143 & 5236 & 175.152 & 5933 & 207.107 & 780 & 249.944 & 728 \\
\hline 130.099 & 4285 & 151.115 & 22603 & 176.070 & 446 & 207.178 & 6780 & 250.232 & 1118 \\
\hline 131.089 & 7407 & 151.151 & 6701 & 176.156 & 2219 & 208.184 & 3898 & 251.218 & 575 \\
\hline 132.094 & 2422 & 151.220 & 663 & 177.130 & 1883 & 208.218 & 1664 & 251.948 & 1258 \\
\hline 133.069 & 1511 & 152.066 & 1491 & 177.167 & 3886 & 209.155 & 1606 & 252.210 & 692 \\
\hline 133.105 & 20470 & 152.122 & 13155 & 178.083 & 752 & 209.192 & 2201 & 253.221 & 447 \\
\hline 133.207 & 603 & 152.159 & 9355 & 178.136 & 1179 & \begin{tabular}{|l|}
210.165 \\
\end{tabular} & 2219 & 254.229 & 472 \\
\hline 134.112 & 11297 & 153.094 & 3274 & 178.174 & 2274 & \begin{tabular}{|l|}
210.239 \\
\end{tabular} & 464 & 255.234 & 398 \\
\hline 135.084 & 9813 & 153.131 & 12659 & 179.091 & 908 & 211.169 & 1146 & 256.242 & 2723 \\
\hline 135.120 & 21195 & 153.167 & 3864 & 179.147 & 8513 & 211.244 & 579 & 257.247 & 638 \\
\hline 135.173 & 561 & 154.102 & 5290 & 179.182 & 2520 & \begin{tabular}{|l|}
212.174 \\
\end{tabular} & 531 & 261.151 & 339 \\
\hline 135.215 & 602 & 154.137 & 2142 & 180.153 & 4245 & 213.188 & 8751 & 262.234 & 1173 \\
\hline 136.091 & 8599 & 154.175 & 4721 & 180.190 & 10385 & \begin{tabular}{|l|}
214.193 \\
\end{tabular} & 2158 & 263.167 & 3769 \\
\hline 136.127 & 9415 & 155.110 & 4971 & 181.126 & 3712 & \begin{tabular}{|l|}
215.200 \\
\end{tabular} & 428 & 263.239 & 913 \\
\hline 137.056 & 1151 & 155.147 & 1354 & 181.161 & 3958 & \begin{tabular}{|l|}
217.199 \\
\end{tabular} & 1359 & 264.174 & 1376 \\
\hline 137.100 & 36620 & 155.182 & 1694 & 181.194 & 2593 & \begin{tabular}{|l|}
218.208 \\
\end{tabular} & 818 & 264.248 & 45340 \\
\hline 137.136 & 13646 & 156.117 & 1835 & 182.133 & 3447 & \begin{tabular}{|l|}
218.950 \\
\end{tabular} & 347 & 264.374 & 1993 \\
\hline 137.206 & 1301 & 157.126 & 7065 & 182.169 & 2267 & 219.175 & 861 & 264.897 & 545 \\
\hline 137.540 & 396 & 158.130 & 1912 & 182.206 & 1209 & \begin{tabular}{|l|}
220.144 \\
\end{tabular} & 559 & 265.252 & 10843 \\
\hline 138.035 & 349 & 159.083 & 544 & 183.141 & 1853 & \begin{tabular}{|l|}
220.222 \\
\end{tabular} & 14236 & 265.366 & 548 \\
\hline 138.107 & 23517 & 159.121 & 2036 & 183.177 & 1705 & 221.193 & 11984 & 265.444 & 514 \\
\hline 138.144 & 15604 & 160.126 & 807 & 184.150 & 1007 & \begin{tabular}{|l|}
222.200 \\
\end{tabular} & 4814 & 266.257 & 1615 \\
\hline 138.208 & 1102 & 161.100 & 1285 & 185.157 & 7748 & \begin{tabular}{|l|}
222.237 \\
\end{tabular} & 11660 & 270.259 & 1208 \\
\hline 139.016 & 243 & 161.136 & 8445 & 186.164 & 3110 & 223.175 & 1176 & 276.209 & 430 \\
\hline 139.079 & 2401 & 162.142 & 3838 & 187.158 & 1636 & \begin{tabular}{|l|}
223.207 \\
\end{tabular} & 1811 & 278.225 & 1628 \\
\hline 139.115 & 22190 & 163.115 & 3492 & 189.167 & 3493 & \begin{tabular}{|l|}
223.239 \\
\end{tabular} & 2824 & 280.242 & 7733 \\
\hline 139.151 & 7374 & 163.152 & 6165 & 190.172 & 1236 & \begin{tabular}{|l|}
224.176 \\
\end{tabular} & 997 & 281.248 & 1745 \\
\hline 139.237 & 412 & 164.122 & 3215 & 191.146 & 1421 & \begin{tabular}{|l|}
225.188 \\
\end{tabular} & 945 & 282.257 & 3288 \\
\hline 140.087 & 9069 & 164.159 & 3469 & 191.183 & 1985 & \begin{tabular}{|l|}
225.224 \\
\end{tabular} & 2570 & 283.262 & 503 \\
\hline 140.121 & 3118 & 165.079 & 1229 & 192.154 & 1043 & \begin{tabular}{|l|}
226.229 \\
\end{tabular} & 559 & 284.273 & 703 \\
\hline 140.159 & 8332 & 165.131 & 13510 & 192.190 & 1814 & 227.203 & 4665 & & \\
\hline 141.094 & 9853 & 165.167 & 3541 & 193.162 & 6303 & \begin{tabular}{|l|}
228.206 \\
\end{tabular} & 1111 & & \\
\hline 141.130 & 1055 & 166.138 & 6085 & 193.197 & 1652 & 233.220 & 384 & & \\
\hline
\end{tabular}


Table S15. High-resolution mass table for the compound identified as docosene.

\begin{tabular}{|c|c|c|c|c|c|c|c|c|c|}
\hline Mass & $\begin{array}{c}\text { Ion } \\
\text { counts }\end{array}$ & Mass & $\begin{array}{c}\text { Ion } \\
\text { counts }\end{array}$ & Mass & $\begin{array}{c}\text { Ion } \\
\text { counts }\end{array}$ & Mass & $\begin{array}{c}\text { Ion } \\
\text { counts }\end{array}$ & Mass & $\begin{array}{c}\text { Ion } \\
\text { counts }\end{array}$ \\
\hline 50.019 & 712 & 69.036 & 801 & 85.067 & 1219 & 111.187 & 808 & 140.159 & 3266 \\
\hline 51.026 & 1556 & 69.073 & 77734 & 85.104 & 28735 & 112.091 & 1999 & 141.085 & 535 \\
\hline 52.034 & 922 & 69.349 & 425 & 85.177 & 735 & 112.127 & 9005 & 141.166 & 1283 \\
\hline 53.042 & 6744 & 69.373 & 273 & 86.041 & 373 & 113.064 & 529 & 143.110 & 1082 \\
\hline 54.050 & 16284 & 69.399 & 506 & 86.108 & 2122 & 113.096 & 458 & 145.105 & 389 \\
\hline 54.108 & 420 & 69.454 & 412 & 87.048 & 3077 & 113.136 & 3376 & 147.082 & 311 \\
\hline 55.021 & 7071 & 69.667 & 489 & 88.054 & 419 & 114.072 & 1040 & 147.119 & 397 \\
\hline 55.057 & 107558 & 69.722 & 408 & 89.047 & 332 & 114.139 & 358 & 149.134 & 599 \\
\hline 55.097 & 1036 & 69.945 & 449 & 91.058 & 4157 & 115.058 & 986 & 151.115 & 645 \\
\hline 55.298 & 491 & 70.045 & 1416 & 92.064 & 1199 & 115.078 & 1978 & 151.151 & 499 \\
\hline 55.326 & 776 & 70.081 & 52699 & 93.073 & 3232 & 116.084 & 767 & 152.159 & 1411 \\
\hline 55.360 & 830 & 70.392 & 326 & 94.080 & 2236 & 117.074 & 1252 & 153.167 & 2637 \\
\hline 55.384 & 597 & 70.444 & 427 & 95.053 & 1081 & 118.078 & 437 & 154.175 & 2044 \\
\hline 55.544 & 413 & 70.718 & 375 & 95.089 & 10159 & 119.088 & 1054 & 155.182 & 744 \\
\hline 55.582 & 680 & 71.052 & 1949 & 96.060 & 2144 & 120.092 & 651 & 157.123 & 791 \\
\hline 55.628 & 457 & 71.089 & 52768 & 96.097 & 12328 & 121.068 & 400 & 161.127 & 349 \\
\hline 55.815 & 486 & 71.429 & 438 & 97.068 & 3602 & 121.104 & 1604 & 165.162 & 370 \\
\hline 55.848 & 322 & 71.463 & 424 & 97.104 & 67674 & 122.074 & 415 & 166.174 & 739 \\
\hline 56.027 & 1159 & 71.746 & 386 & 97.470 & 606 & 122.110 & 652 & 167.084 & 321 \\
\hline 56.065 & 46063 & 72.059 & 512 & 97.517 & 465 & 123.083 & 1951 & 167.182 & 1463 \\
\hline 56.123 & 1566 & 72.092 & 2939 & 97.772 & 429 & 123.120 & 2011 & 168.189 & 1592 \\
\hline 56.315 & 537 & 73.032 & 14603 & 97.812 & 329 & 124.092 & 1151 & 169.194 & 468 \\
\hline 56.353 & 478 & 73.068 & 1114 & 97.850 & 438 & 124.128 & 3008 & 171.142 & 998 \\
\hline 56.557 & 412 & 73.104 & 354 & 98.076 & 6371 & 125.067 & 389 & 180.193 & 485 \\
\hline 56.589 & 449 & 74.039 & 1835 & 98.111 & 15101 & 125.100 & 1372 & 181.110 & 445 \\
\hline 57.037 & 4695 & 75.028 & 518 & 98.169 & 727 & 125.136 & 12768 & 181.197 & 798 \\
\hline 57.073 & 98762 & 76.035 & 295 & 98.452 & 436 & 125.210 & 386 & 182.205 & 1243 \\
\hline 57.317 & 736 & 77.042 & 3206 & 99.046 & 627 & 126.070 & 484 & 185.157 & 1333 \\
\hline 57.347 & 607 & 78.049 & 1830 & 99.082 & 1171 & 126.142 & 4964 & 194.206 & 544 \\
\hline 57.373 & 803 & 79.058 & 6902 & 99.120 & 6206 & 127.079 & 727 & 195.216 & 500 \\
\hline 57.411 & 388 & 80.065 & 3525 & 100.056 & 752 & 127.151 & 1976 & 196.221 & 761 \\
\hline 57.607 & 635 & 81.037 & 712 & 100.122 & 641 & 128.066 & 959 & 199.168 & 663 \\
\hline 57.636 & 491 & 81.073 & 17639 & 101.063 & 2330 & 129.094 & 4311 & 208.223 & 346 \\
\hline 57.853 & 440 & 82.044 & 428 & 102.069 & 336 & 130.097 & 540 & 209.225 & 359 \\
\hline 58.044 & 1194 & 82.081 & 23896 & 103.060 & 392 & 131.093 & 694 & 210.237 & 448 \\
\hline 58.077 & 4488 & 82.149 & 520 & 104.065 & 525 & 133.105 & 806 & 213.187 & 1509 \\
\hline 59.053 & 1724 & 83.052 & 3350 & 105.038 & 426 & 134.108 & 408 & 214.194 & 240 \\
\hline 60.024 & 11190 & 83.089 & 81591 & 105.073 & 1943 & 135.082 & 690 & 218.949 & 211 \\
\hline 61.032 & 3166 & 83.167 & 1541 & 106.079 & 612 & 135.120 & 752 & 222.239 & 375 \\
\hline 63.026 & 499 & 83.386 & 435 & 107.052 & 523 & 136.088 & 558 & 224.258 & 334 \\
\hline 65.042 & 2330 & 83.417 & 417 & 107.088 & 1719 & 136.126 & 314 & 227.204 & 579 \\
\hline 66.050 & 2106 & 83.450 & 433 & 108.095 & 1081 & 137.049 & 438 & 238.269 & 296 \\
\hline 67.058 & 25743 & 83.486 & 468 & 109.068 & 1264 & 137.099 & 1308 & 241.218 & 1088 \\
\hline 67.097 & 387 & 83.760 & 449 & 109.104 & 4214 & 137.136 & 807 & 251.945 & 664 \\
\hline 67.129 & 542 & 84.060 & 4534 & 110.076 & 1547 & 138.106 & 681 & 264.245 & 651 \\
\hline 68.029 & 431 & 84.096 & 25548 & 110.112 & 5057 & 138.143 & 1861 & 280.314 & 448 \\
\hline 68.065 & 18165 & 84.164 & 617 & 111.084 & 2904 & 139.116 & 1006 & 284.270 & 509 \\
\hline 68.130 & 387 & 85.038 & 426 & 111.120 & 29870 & 139.151 & 4321 & 308.349 & 627 \\
\hline
\end{tabular}


Table S16. High-resolution mass table for the compound identified as octadecanoic acid.

\begin{tabular}{|c|c|c|c|c|c|c|c|c|c|}
\hline Mass & $\begin{array}{c}\text { Ion } \\
\text { counts }\end{array}$ & Mass & $\begin{array}{c}\text { Ion } \\
\text { counts }\end{array}$ & Mass & $\begin{array}{c}\text { Ion } \\
\text { counts }\end{array}$ & Mass & $\begin{array}{c}\text { Ion } \\
\text { counts }\end{array}$ & Mass & $\begin{array}{c}\text { Ion } \\
\text { counts }\end{array}$ \\
\hline 50.019 & 563 & 69.671 & 419 & 95.052 & 1663 & 122.111 & 1106 & 152.159 & 935 \\
\hline 51.026 & 1654 & 69.721 & 420 & 95.089 & 13157 & 123.084 & 3120 & 153.130 & 1224 \\
\hline 52.034 & 1092 & 70.044 & 2931 & 95.162 & 399 & 123.120 & 2337 & 153.166 & 457 \\
\hline 53.042 & 7326 & 70.080 & 17964 & 96.060 & 3905 & 124.092 & 2174 & 154.174 & 567 \\
\hline 54.050 & 13201 & 70.148 & 585 & 96.096 & 9044 & 124.127 & 2214 & 155.108 & 452 \\
\hline 55.021 & 12197 & 71.052 & 4494 & 97.068 & 9983 & 125.099 & 3735 & 155.181 & 1026 \\
\hline 55.057 & 81585 & 71.089 & 33189 & 97.104 & 21941 & 125.135 & 2951 & 157.124 & 2882 \\
\hline 55.325 & 662 & 71.151 & 765 & \begin{tabular}{|c|}
97.151 \\
\end{tabular} & 430 & 126.072 & 520 & 158.133 & 737 \\
\hline 55.348 & 568 & 71.188 & 415 & \begin{tabular}{|c|}
97.189 \\
\end{tabular} & 607 & 126.105 & 668 & 159.095 & 417 \\
\hline 55.371 & 575 & 72.058 & 837 & 98.076 & 15198 & 126.143 & 1590 & 161.134 & 594 \\
\hline 55.542 & 406 & 72.092 & 1945 & 98.111 & 5482 & 127.078 & 1698 & 163.116 & 738 \\
\hline 55.576 & 505 & 73.032 & 72685 & 98.162 & 488 & 127.151 & 2036 & 163.150 & 759 \\
\hline 55.602 & 504 & 73.068 & 4016 & 99.047 & 1218 & 128.069 & 1180 & 164.150 & 395 \\
\hline 55.814 & 429 & 73.312 & 437 & 99.082 & 2334 & 129.040 & 1031 & 165.076 & 435 \\
\hline 56.028 & 1495 & 73.341 & 287 & \begin{tabular}{|c|}
99.120 \\
\end{tabular} & 5011 & 129.094 & 24003 & 165.131 & 974 \\
\hline 56.065 & 23927 & 73.368 & 557 & 100.055 & 1813 & 129.185 & 590 & 166.174 & 946 \\
\hline 56.123 & 759 & 73.407 & 469 & 100.123 & 510 & 130.099 & 3052 & 167.086 & 388 \\
\hline 56.372 & 375 & 73.629 & 436 & \begin{tabular}{|l|}
101.063 \\
\end{tabular} & 10373 & 131.053 & 583 & 167.145 & 764 \\
\hline 57.037 & 8104 & 73.672 & 426 & 101.099 & 405 & 131.089 & 1464 & 168.178 & 493 \\
\hline 57.073 & 70709 & 74.038 & 6694 & \begin{tabular}{|l|l|}
102.069 \\
\end{tabular} & 2206 & 132.062 & 440 & 169.199 & 524 \\
\hline 57.320 & 382 & 75.030 & 2181 & \begin{tabular}{|l|l|}
103.059 \\
\end{tabular} & 782 & 133.104 & 1690 & 171.140 & 4398 \\
\hline 57.346 & 442 & 76.030 & 421 & 104.064 & 628 & 134.111 & 680 & 172.147 & 1031 \\
\hline 57.368 & 579 & 77.042 & 4489 & 105.039 & 353 & 135.083 & 1353 & 173.113 & 418 \\
\hline 57.399 & 430 & 78.049 & 2474 & 105.073 & 2889 & 135.120 & 2258 & 175.152 & 359 \\
\hline 57.580 & 440 & 79.058 & 10030 & 106.079 & 1059 & 136.090 & 676 & 177.167 & 602 \\
\hline 57.614 & 548 & 80.065 & 4920 & 107.052 & 820 & 136.126 & 834 & 179.179 & 444 \\
\hline 57.830 & 409 & 81.037 & 980 & \begin{tabular}{|l|l|}
107.087 \\
\end{tabular} & 3377 & 137.099 & 2386 & 180.190 & 1046 \\
\hline 57.889 & 389 & 81.073 & 19729 & 108.096 & 1823 & 137.136 & 984 & 181.125 & 471 \\
\hline 58.044 & 2002 & 81.144 & 459 & 109.068 & 2147 & 138.105 & 1244 & 181.169 & 432 \\
\hline 58.076 & 3409 & 82.044 & 978 & 109.105 & 6008 & 138.142 & 1290 & 182.165 & 407 \\
\hline 59.052 & 4571 & 82.081 & 14155 & 110.076 & 3060 & 139.114 & 2300 & 185.157 & 9609 \\
\hline 60.007 & 163 & 83.053 & 7642 & 110.112 & 4065 & 139.151 & 1011 & 186.161 & 1737 \\
\hline 60.024 & 61249 & 83.089 & 33659 & 111.084 & 6197 & 140.088 & 495 & 187.165 & 365 \\
\hline 60.305 & 427 & 83.155 & 926 & 111.120 & 8345 & 140.121 & 366 & 191.185 & 406 \\
\hline 60.326 & 276 & 84.060 & 10549 & 112.091 & 4279 & 140.158 & 1047 & 192.198 & 445 \\
\hline 60.361 & 394 & 84.096 & 8891 & 112.127 & 3087 & 141.085 & 1180 & 193.163 & 528 \\
\hline 60.561 & 399 & 85.032 & 645 & 113.063 & 1508 & 141.167 & 1260 & 194.207 & 382 \\
\hline 61.031 & 17820 & 85.068 & 2953 & \begin{tabular}{|l|}
113.099 \\
\end{tabular} & 732 & 142.084 & 455 & 195.185 & 367 \\
\hline 61.097 & 647 & 85.104 & 19977 & 113.136 & 2938 & 143.110 & 4223 & 199.172 & 3919 \\
\hline 62.034 & 900 & 85.169 & 501 & 114.071 & 2324 & 144.115 & 885 & 200.175 & 640 \\
\hline 63.027 & 405 & 86.040 & 660 & \begin{tabular}{|l|}
114.141 \\
\end{tabular} & 300 & 145.069 & 763 & 205.193 & 338 \\
\hline 65.042 & 3029 & 86.074 & 431 & 115.056 & 1057 & 145.104 & 485 & 207.176 & 370 \\
\hline 66.050 & 2274 & 86.108 & 1628 & 115.078 & 9024 & 147.083 & 682 & 213.187 & 2487 \\
\hline 67.057 & 25111 & 87.047 & 13438 & 116.085 & 3921 & 147.120 & 779 & 214.192 & 511 \\
\hline 67.124 & 676 & 87.084 & 784 & \begin{tabular}{|l|}
117.041 \\
\end{tabular} & 1246 & 148.128 & 559 & 220.220 & 740 \\
\hline 68.029 & 399 & 88.055 & 6363 & 117.073 & 1720 & 149.100 & 1625 & 221.195 & 476 \\
\hline 68.065 & 14935 & 89.063 & 1183 & 118.080 & 438 & 149.136 & 1505 & 222.236 & 1532 \\
\hline 69.037 & 1840 & 91.058 & 5339 & 119.088 & 1879 & 150.107 & 606 & 227.203 & 3194 \\
\hline 69.073 & 52570 & 92.064 & 1604 & \begin{tabular}{|l|}
120.094 \\
\end{tabular} & 830 & 150.139 & 459 & 228.209 & 804 \\
\hline 69.140 & 1635 & 93.073 & 4890 & \begin{tabular}{|l|}
121.068 \\
\end{tabular} & 1343 & 151.114 & 1332 & 236.214 & 378 \\
\hline 69.177 & 526 & 94.043 & 416 & \begin{tabular}{|l|}
121.104 \\
\end{tabular} & 2959 & 151.152 & 551 & 241.218 & 10614 \\
\hline 69.380 & 428 & 94.080 & 3708 & 122.076 & 573 & 152.123 & 662 & 242.222 & 2035 \\
\hline
\end{tabular}


Table S16 (continued). High-resolution mass table for the compound identified as octadecanoic acid.

\begin{tabular}{|c|c|c|c|c|c|c|c|c|c|}
\hline Mass & $\begin{array}{c}\text { Ion } \\
\text { counts }\end{array}$ & Mass & $\begin{array}{c}\text { Ion } \\
\text { counts }\end{array}$ & Mass & $\begin{array}{c}\text { Ion } \\
\text { counts }\end{array}$ & Mass & $\begin{array}{c}\text { Ion } \\
\text { counts }\end{array}$ & Mass & $\begin{array}{c}\text { Ion } \\
\text { counts }\end{array}$ \\
\hline 249.942 & 362 & 256.240 & 757 & 264.246 & 1810 & 269.250 & 524 & 284.272 & 5522 \\
\hline 251.942 & 504 & 263.167 & 1970 & 265.251 & 435 & 278.190 & 362 & 285.277 & 1309 \\
\hline 255.234 & 2200 & 264.173 & 591 & 267.270 & 370 & 280.240 & 377 & & \\
\hline
\end{tabular}


Table S17. High-resolution mass table for the compound identified as eicosanol.

\begin{tabular}{|c|c|c|c|c|c|c|c|c|c|}
\hline Mass & $\begin{array}{c}\text { Ion } \\
\text { counts }\end{array}$ & Mass & $\begin{array}{c}\text { Ion } \\
\text { counts }\end{array}$ & Mass & $\begin{array}{c}\text { Ion } \\
\text { counts }\end{array}$ & Mass & $\begin{array}{c}\text { Ion } \\
\text { counts }\end{array}$ & Mass & $\begin{array}{c}\text { Ion } \\
\text { counts }\end{array}$ \\
\hline 50.018 & 464 & 69.037 & 896 & 91.057 & 3316 & 116.087 & 721 & 150.134 & 374 \\
\hline 51.026 & 1152 & 69.073 & 73529 & 92.064 & 1164 & 117.040 & 565 & 151.114 & 466 \\
\hline 52.034 & 816 & 69.354 & 350 & 93.073 & 3618 & 117.074 & 831 & 151.152 & 775 \\
\hline 53.042 & 5917 & 69.373 & 348 & 94.045 & 334 & 118.077 & 310 & 152.068 & 338 \\
\hline 54.050 & 15395 & 69.402 & 523 & 94.081 & 2442 & 119.089 & 1036 & 152.160 & 1515 \\
\hline 54.109 & 394 & 69.694 & 424 & 95.053 & 955 & 120.094 & 607 & 153.166 & 1655 \\
\hline 55.021 & 4269 & 70.045 & 1384 & 95.089 & 17595 & 121.068 & 437 & 154.173 & 1717 \\
\hline 55.057 & 94300 & 70.080 & 32998 & 96.060 & 1618 & 121.104 & 1834 & 155.181 & 480 \\
\hline 55.297 & 446 & 70.147 & 840 & 96.096 & 19805 & 122.072 & 368 & 157.126 & 496 \\
\hline 55.326 & 477 & 71.052 & 3298 & 96.153 & 303 & 122.111 & 712 & 162.094 & 742 \\
\hline 55.349 & 718 & 71.089 & 34284 & 96.183 & 443 & 123.083 & 1438 & 163.149 & 499 \\
\hline 55.536 & 341 & 71.158 & 818 & 97.068 & 3442 & 123.119 & 3926 & 164.134 & 389 \\
\hline 55.568 & 560 & 72.059 & 806 & 97.104 & 50550 & 124.091 & 1020 & 165.076 & 320 \\
\hline 55.809 & 423 & 72.092 & 1915 & 97.462 & 461 & 124.127 & 4219 & 165.168 & 423 \\
\hline 55.855 & 445 & 73.032 & 11907 & 97.527 & 452 & 125.099 & 1263 & 166.172 & 1058 \\
\hline 56.028 & 840 & 73.068 & 2092 & 98.076 & 5102 & 125.135 & 8821 & 167.181 & 1168 \\
\hline 56.065 & 36226 & 74.038 & 1410 & 98.111 & 11816 & 126.072 & 370 & 168.190 & 1127 \\
\hline 56.125 & 1339 & 75.031 & 1036 & 98.164 & 425 & 126.143 & 4370 & 169.193 & 348 \\
\hline 56.159 & 391 & 76.034 & 298 & 99.046 & 490 & 127.079 & 784 & 171.140 & 706 \\
\hline 56.307 & 431 & 77.042 & 3150 & 99.083 & 1289 & 127.149 & 1342 & 180.189 & 821 \\
\hline 56.338 & 436 & 78.050 & 1541 & 99.119 & 4181 & 128.065 & 723 & 181.197 & 511 \\
\hline 56.559 & 382 & 79.057 & 7001 & 100.054 & 775 & 129.093 & 4101 & 182.205 & 855 \\
\hline 56.590 & 405 & 80.065 & 3867 & 100.113 & 380 & 130.083 & 482 & 185.156 & 1602 \\
\hline 57.037 & 6730 & 81.037 & 649 & 101.063 & 2026 & 131.089 & 760 & 194.207 & 413 \\
\hline 57.073 & 74175 & 81.073 & 23786 & 102.069 & 444 & 133.104 & 823 & 195.216 & 389 \\
\hline 57.315 & 456 & 81.140 & 564 & 103.057 & 468 & 134.106 & 725 & 196.224 & 512 \\
\hline 57.347 & 587 & 82.044 & 680 & 104.063 & 382 & 135.085 & 696 & 199.170 & 848 \\
\hline 57.367 & 680 & 82.081 & 35991 & 105.038 & 394 & 135.120 & 1363 & 208.218 & 329 \\
\hline 57.407 & 338 & 82.152 & 837 & 105.073 & 1837 & 136.093 & 424 & 210.236 & 378 \\
\hline 57.577 & 426 & 83.052 & 2556 & 106.073 & 1170 & 136.127 & 587 & 213.187 & 639 \\
\hline 57.605 & 347 & 83.089 & 67832 & 107.084 & 3076 & 137.046 & 365 & 222.235 & 551 \\
\hline 57.633 & 432 & 83.388 & 409 & 108.095 & 1427 & 137.100 & 992 & 224.254 & 548 \\
\hline 57.851 & 405 & 83.425 & 489 & 109.067 & 1114 & 137.135 & 2169 & 227.201 & 265 \\
\hline 58.044 & 1697 & 83.711 & 399 & 109.104 & 8032 & 138.107 & 579 & 241.219 & 1037 \\
\hline 58.077 & 3532 & 84.060 & 3883 & 110.076 & 1442 & 138.144 & 2570 & 251.950 & 280 \\
\hline 58.114 & 430 & 84.096 & 20203 & 110.112 & 7255 & 139.115 & 974 & 252.283 & 577 \\
\hline 59.053 & 2147 & 84.154 & 407 & 111.083 & 2430 & 139.150 & 3245 & 255.234 & 686 \\
\hline 60.024 & 10487 & 85.068 & 2383 & 111.120 & 21988 & 140.158 & 2624 & 263.163 & 371 \\
\hline 61.032 & 3216 & 85.104 & 18386 & 111.201 & 569 & 141.087 & 452 & 264.243 & 449 \\
\hline 63.027 & 538 & 85.177 & 483 & 112.091 & 1556 & 141.164 & 801 & 278.292 & 365 \\
\hline 65.042 & 2106 & 86.075 & 431 & 112.127 & 7319 & 143.109 & 874 & 284.277 & 363 \\
\hline 66.049 & 2659 & 86.108 & 1378 & 113.061 & 593 & 145.106 & 403 & 298.284 & 269 \\
\hline 67.057 & 29505 & 87.047 & 2698 & 113.101 & 698 & 147.120 & 476 & & \\
\hline 67.125 & 660 & 87.084 & 449 & 113.135 & 2063 & 148.122 & 374 & & \\
\hline 68.028 & 285 & 88.047 & 414 & 114.070 & 1002 & 149.095 & 558 & & \\
\hline 68.065 & 29755 & 89.044 & 407 & 115.078 & 2638 & 149.137 & 897 & & \\
\hline
\end{tabular}


Table S18. High-resolution mass table for the compound identified as eicosenoic acid.

\begin{tabular}{|c|c|c|c|c|c|c|c|c|c|}
\hline Mass & $\begin{array}{c}\text { Ion } \\
\text { counts }\end{array}$ & Mass & $\begin{array}{c}\text { Ion } \\
\text { counts }\end{array}$ & Mass & $\begin{array}{c}\text { Ion } \\
\text { counts }\end{array}$ & Mass & $\begin{array}{c}\text { Ion } \\
\text { counts }\end{array}$ & Mass & $\begin{array}{c}\text { Ion } \\
\text { counts }\end{array}$ \\
\hline 50.018 & 670 & 78.049 & 2075 & 100.055 & 1825 & 126.142 & 1166 & 154.175 & 317 \\
\hline 51.025 & 1174 & 79.057 & 9737 & 101.063 & 3115 & 127.078 & 1973 & 155.108 & 1072 \\
\hline 52.034 & 938 & 79.117 & 378 & 102.063 & 407 & 128.073 & 1111 & 156.121 & 434 \\
\hline 53.042 & 4997 & 80.065 & 5106 & 103.042 & 3955 & 129.072 & 1266 & 157.119 & 486 \\
\hline 54.049 & 11517 & 81.037 & 1060 & 104.065 & 1009 & 129.093 & 2214 & 158.098 & 406 \\
\hline 55.021 & 6804 & 81.073 & 18255 & 105.036 & 468 & 130.087 & 478 & 159.083 & 389 \\
\hline 55.057 & 46288 & 81.147 & 478 & 105.073 & 2648 & 131.057 & 461 & 161.135 & 911 \\
\hline 55.117 & 1224 & 82.044 & 1437 & 106.078 & 1475 & 131.088 & 867 & 162.094 & 574 \\
\hline 55.332 & 413 & 82.080 & 11978 & 107.053 & 789 & 132.093 & 411 & 163.152 & 688 \\
\hline 55.363 & 421 & 82.148 & 366 & 107.087 & 3356 & 133.104 & 1648 & 164.122 & 463 \\
\hline 56.028 & 1179 & 83.052 & 5146 & 108.096 & 2172 & 134.073 & 390 & 165.077 & 414 \\
\hline 56.065 & 13413 & 83.088 & 22171 & 109.068 & 2061 & 134.108 & 1002 & 165.129 & 880 \\
\hline 56.126 & 422 & 83.162 & 544 & 109.104 & 5420 & 135.082 & 1087 & 166.136 & 569 \\
\hline 57.037 & 6197 & 84.060 & 7384 & 110.075 & 2786 & 135.119 & 1817 & 167.145 & 616 \\
\hline 57.073 & 22174 & 84.095 & 6140 & 110.111 & 3171 & 136.092 & 717 & 171.130 & 433 \\
\hline 57.129 & 751 & 85.030 & 461 & 111.083 & 4536 & 136.126 & 803 & 175.148 & 517 \\
\hline 58.044 & 2035 & 85.067 & 4074 & 111.120 & 5443 & 137.047 & 494 & 177.166 & 415 \\
\hline 58.076 & 1307 & 85.104 & 4795 & 112.090 & 2240 & 137.099 & 2803 & 179.145 & 573 \\
\hline 59.052 & 2073 & 86.039 & 402 & 112.127 & 2009 & 137.135 & 1085 & 180.148 & 387 \\
\hline 60.023 & 8134 & 86.073 & 418 & 113.063 & 1633 & 138.106 & 1789 & 180.190 & 416 \\
\hline 61.031 & 2274 & 86.106 & 347 & 113.098 & 1234 & 138.142 & 972 & 181.148 & 404 \\
\hline 63.026 & 354 & 87.046 & 2796 & 113.134 & 655 & 139.114 & 2067 & 182.131 & 391 \\
\hline 65.042 & 2531 & 87.081 & 433 & 114.070 & 2488 & 139.149 & 633 & 185.156 & 671 \\
\hline 66.050 & 1822 & 88.053 & 388 & 115.057 & 1298 & 140.085 & 540 & 193.161 & 486 \\
\hline 67.057 & 22752 & 89.041 & 445 & 115.078 & 1859 & 140.121 & 343 & 194.206 & 506 \\
\hline 67.118 & 486 & 91.057 & 5566 & 116.065 & 805 & 140.160 & 574 & 199.173 & 511 \\
\hline 68.065 & 13017 & 92.063 & 1677 & 117.072 & 1536 & 141.093 & 1198 & 208.215 & 599 \\
\hline 69.037 & 1418 & 93.073 & 5531 & 118.078 & 530 & 143.106 & 637 & 211.210 & 851 \\
\hline 69.073 & 31239 & 94.049 & 343 & 119.088 & 1840 & 145.104 & 424 & 213.185 & 392 \\
\hline 69.140 & 695 & 94.080 & 3978 & 120.096 & 650 & 147.082 & 404 & 221.194 & 355 \\
\hline 70.044 & 1649 & 95.053 & 1676 & 121.067 & 986 & 147.120 & 1173 & 235.209 & 411 \\
\hline 70.080 & 11411 & 95.088 & 13313 & 121.103 & 2601 & 148.125 & 579 & 241.218 & 434 \\
\hline 71.052 & 4036 & 96.059 & 4361 & 122.075 & 605 & 149.096 & 914 & 248.250 & 391 \\
\hline 71.088 & 9308 & 96.096 & 7743 & 122.111 & 1468 & 149.135 & 1115 & 249.224 & 498 \\
\hline 72.059 & 693 & 97.067 & 6253 & 123.083 & 3127 & 150.103 & 677 & 250.248 & 493 \\
\hline 72.093 & 546 & 97.104 & 14069 & 123.120 & 2281 & 151.113 & 1393 & 251.941 & 403 \\
\hline 73.031 & 9189 & 97.173 & 453 & 124.090 & 2127 & 151.151 & 521 & 263.166 & 274 \\
\hline 73.068 & 1230 & 98.075 & 8042 & 124.127 & 1669 & 152.061 & 346 & 264.244 & 315 \\
\hline 74.039 & 2092 & 98.111 & 3626 & 125.099 & 2722 & 152.120 & 940 & 278.243 & 394 \\
\hline 75.033 & 498 & 99.047 & 1661 & 125.135 & 1931 & 152.159 & 678 & 292.277 & 2312 \\
\hline 76.032 & 310 & 99.082 & 1777 & 126.064 & 753 & 153.131 & 1151 & 293.280 & 649 \\
\hline 77.042 & 4039 & 99.119 & 1025 & 126.105 & 641 & 154.096 & 449 & & \\
\hline
\end{tabular}


Table S19. High-resolution mass table for the compound identified as squalene.

\begin{tabular}{|c|c|c|c|c|c|c|c|c|c|}
\hline Mass & $\begin{array}{c}\text { Ion } \\
\text { counts }\end{array}$ & Mass & $\begin{array}{c}\text { Ion } \\
\text { counts }\end{array}$ & Mass & $\begin{array}{c}\text { Ion } \\
\text { counts }\end{array}$ & Mass & $\begin{array}{c}\text { Ion } \\
\text { counts }\end{array}$ & Mass & $\begin{array}{c}\text { Ion } \\
\text { counts }\end{array}$ \\
\hline 49.010 & 625 & 67.353 & 2357 & 72.060 & 1171 & 81.445 & 20081 & 93.457 & 2384 \\
\hline 50.018 & 3553 & 67.389 & 2289 & 72.092 & 2968 & 81.495 & 9528 & 93.550 & 295 \\
\hline 51.026 & 7980 & 67.427 & 1649 & 72.142 & 1586 & 81.527 & 3179 & 93.755 & 1861 \\
\hline 52.033 & 5073 & 67.461 & 450 & 72.372 & 856 & 81.547 & 2580 & 94.042 & 1529 \\
\hline 53.026 & 136 & 67.639 & 2565 & 72.400 & 1061 & 81.718 & 14775 & 94.080 & 98674 \\
\hline 53.042 & 102821 & 67.674 & 2489 & 72.427 & 981 & 82.013 & 10375 & 94.335 & 267 \\
\hline 53.284 & 493 & 67.754 & 888 & 72.667 & 966 & 82.052 & 3833 & 94.388 & 1221 \\
\hline 53.305 & 642 & 67.887 & 2300 & 72.950 & 461 & 82.078 & 174458 & 94.432 & 1184 \\
\hline 53.323 & 718 & 67.946 & 2030 & 73.031 & 3137 & 82.127 & 2475 & 94.460 & 1374 \\
\hline 53.356 & 610 & 67.992 & 833 & 73.234 & 408 & 82.315 & 6577 & 94.725 & 1314 \\
\hline 53.568 & 566 & 68.034 & 1082 & 74.018 & 341 & 82.402 & 2365 & 94.770 & 781 \\
\hline 53.804 & 469 & 68.065 & 353393 & 74.038 & 539 & 82.505 & 694 & 94.807 & 616 \\
\hline 54.015 & 376 & 68.339 & 1697 & 75.028 & 762 & 82.595 & 4558 & 94.960 & 402 \\
\hline 54.048 & 11769 & 68.363 & 1959 & 76.033 & 482 & 82.643 & 3915 & 95.011 & 795 \\
\hline 54.111 & 388 & 68.391 & 3006 & 77.042 & 52950 & 82.885 & 2803 & 95.088 & 398892 \\
\hline 55.021 & 2066 & 68.421 & 3176 & 77.326 & 529 & 82.924 & 2769 & 95.140 & 3939 \\
\hline 55.057 & 183687 & 68.452 & 1094 & 77.374 & 511 & 83.053 & 1048 & 95.409 & 2524 \\
\hline 55.299 & 1174 & 68.473 & 1231 & 77.420 & 440 & 83.088 & 67316 & 95.441 & 2035 \\
\hline 55.325 & 1165 & 68.675 & 3602 & 78.048 & 10224 & 83.165 & 3588 & 95.471 & 3532 \\
\hline 55.352 & 1635 & 68.709 & 3050 & 79.057 & 111970 & 83.217 & 2956 & 95.516 & 2275 \\
\hline 55.552 & 777 & 68.760 & 1655 & 79.335 & 2623 & 83.420 & 997 & 95.571 & 374 \\
\hline 55.598 & 1308 & 68.875 & 3049 & 79.379 & 1540 & 83.472 & 2157 & 95.746 & 2096 \\
\hline 55.812 & 758 & 68.929 & 4566 & 79.416 & 1366 & 83.755 & 1275 & 95.778 & 2284 \\
\hline 55.848 & 817 & 68.963 & 4953 & 79.442 & 1330 & 83.794 & 1263 & 95.815 & 2434 \\
\hline 56.027 & 605 & 69.043 & 6536 & 79.496 & 632 & 84.059 & 2168 & 96.062 & 2000 \\
\hline 56.063 & 15881 & 69.073 & 890819 & 79.624 & 565 & 84.093 & 6532 & 96.093 & 41248 \\
\hline 56.121 & 754 & 69.087 & 642990 & 79.661 & 987 & 84.127 & 632 & 96.141 & 1803 \\
\hline 56.299 & 510 & 69.123 & 136232 & 79.696 & 1146 & 84.321 & 467 & 96.169 & 1901 \\
\hline 56.338 & 543 & 69.325 & 14928 & 79.755 & 509 & 84.378 & 602 & 96.359 & 515 \\
\hline 57.036 & 1803 & 69.374 & 27431 & 79.985 & 640 & 84.427 & 431 & 96.415 & 1769 \\
\hline 57.073 & 56924 & 69.390 & 10717 & 80.065 & 54702 & 84.716 & 429 & 96.459 & 1514 \\
\hline 57.324 & 397 & 69.418 & 30595 & 80.299 & 886 & 85.029 & 306 & 96.695 & 597 \\
\hline 57.347 & 493 & 69.472 & 8103 & 80.325 & 653 & 85.067 & 643 & 96.738 & 1233 \\
\hline 57.397 & 398 & 69.497 & 2530 & 80.357 & 1252 & 85.104 & 4352 & 96.776 & 1100 \\
\hline 57.582 & 408 & 69.670 & 26233 & 80.390 & 862 & 86.107 & 376 & 97.001 & 414 \\
\hline 57.859 & 316 & 69.704 & 22471 & 80.447 & 1745 & 87.048 & 851 & 97.068 & 2161 \\
\hline 58.044 & 591 & 69.945 & 18866 & 80.501 & 1992 & 88.055 & 366 & 97.104 & 34180 \\
\hline 58.076 & 2927 & 70.076 & 197549 & 80.610 & 2506 & 89.042 & 747 & 97.153 & 617 \\
\hline 59.051 & 778 & 70.149 & 11433 & 80.699 & 3466 & 90.048 & 277 & 97.186 & 853 \\
\hline 60.024 & 2516 & 70.199 & 14728 & 80.737 & 3447 & 91.057 & 74690 & 97.363 & 422 \\
\hline 61.011 & 555 & 70.380 & 1045 & 80.815 & 4133 & 91.332 & 927 & 97.429 & 652 \\
\hline 61.031 & 1105 & 70.461 & 10126 & 80.849 & 2969 & 91.401 & 396 & 97.489 & 433 \\
\hline 62.018 & 1073 & 70.495 & 10235 & 80.885 & 4948 & 91.449 & 614 & 97.693 & 416 \\
\hline 63.026 & 2257 & 70.747 & 7602 & 80.925 & 3402 & 92.065 & 40134 & 97.751 & 445 \\
\hline 64.034 & 606 & 71.016 & 5293 & 80.965 & 5836 & 92.143 & 1165 & 98.076 & 2666 \\
\hline 65.042 & 21488 & 71.048 & 5727 & 81.003 & 5671 & 92.374 & 422 & 98.108 & 3363 \\
\hline 65.104 & 595 & 71.088 & 31762 & 81.038 & 6917 & 92.412 & 481 & 99.082 & 536 \\
\hline 65.331 & 414 & 71.118 & 965 & 81.069 & 463667 & 92.984 & 351 & 99.119 & 647 \\
\hline 66.049 & 11186 & 71.281 & 4105 & 81.080 & 737672 & 93.073 & 259398 & 101.062 & 473 \\
\hline 67.037 & 204 & 71.533 & 2202 & 81.123 & 95853 & 93.125 & 2235 & 102.050 & 571 \\
\hline 67.057 & 264348 & 71.587 & 2721 & 81.339 & 13372 & 93.390 & 1660 & 103.057 & 5120 \\
\hline 67.327 & 2138 & 71.839 & 2117 & 81.398 & 20936 & 93.421 & 1471 & 104.064 & 2497 \\
\hline
\end{tabular}


Table S19 (continued). High-resolution mass table for the compound identified as squalene.

\begin{tabular}{|c|c|c|c|c|c|c|c|c|c|}
\hline Mass & $\begin{array}{c}\text { Ion } \\
\text { counts }\end{array}$ & Mass & $\begin{array}{c}\text { Ion } \\
\text { counts }\end{array}$ & Mass & $\begin{array}{c}\text { Ion } \\
\text { counts }\end{array}$ & Mass & $\begin{array}{c}\text { Ion } \\
\text { counts }\end{array}$ & Mass & $\begin{array}{c}\text { Ion } \\
\text { counts }\end{array}$ \\
\hline 105.073 & 56624 & 115.057 & 4051 & 133.104 & 38661 & 149.098 & 1032 & 191.182 & 38312 \\
\hline 105.446 & 438 & 116.064 & 1712 & 134.061 & 453 & 149.135 & 154121 & 191.298 & 1105 \\
\hline 105.501 & 323 & 117.073 & 6331 & 134.111 & 34484 & 149.540 & 902 & 191.354 & 603 \\
\hline 105.535 & 430 & 118.079 & 2179 & 134.996 & 456 & 149.596 & 1875 & 192.188 & 21399 \\
\hline 105.798 & 476 & 119.088 & 68175 & 135.040 & 432 & 149.663 & 1220 & 192.284 & 715 \\
\hline 106.080 & 18304 & 119.447 & 471 & 135.119 & 127872 & 149.950 & 738 & 193.194 & 4091 \\
\hline 107.057 & 950 & 119.481 & 310 & 135.499 & 631 & 150.003 & 875 & 194.199 & 490 \\
\hline 107.088 & 161530 & 119.515 & 432 & 135.540 & 645 & 150.055 & 1066 & 199.152 & 830 \\
\hline 107.172 & 4883 & 119.557 & 448 & 135.577 & 1101 & 150.140 & 27302 & 200.158 & 387 \\
\hline 107.427 & 764 & 119.921 & 476 & 135.627 & 762 & 150.370 & 625 & 201.166 & 5007 \\
\hline 107.463 & 1119 & 120.095 & 22063 & 135.954 & 839 & 150.423 & 545 & 202.173 & 2532 \\
\hline 107.507 & 1579 & 120.178 & 761 & 136.037 & 720 & 150.821 & 479 & 203.182 & 34870 \\
\hline 107.572 & 541 & 120.243 & 636 & 136.127 & 218196 & 151.149 & 7855 & 203.294 & 1009 \\
\hline 107.793 & 779 & 120.919 & 478 & 136.511 & 955 & 151.226 & 556 & 204.187 & 11063 \\
\hline 107.839 & 1099 & 121.104 & 260187 & 136.549 & 1036 & 152.063 & 517 & 204.282 & 502 \\
\hline 108.095 & 40514 & 121.467 & 1343 & 136.593 & 2009 & 152.154 & 1032 & 205.197 & 22398 \\
\hline 108.179 & 2139 & 121.501 & 1384 & 136.648 & 1525 & 153.072 & 347 & 205.292 & 772 \\
\hline 108.439 & 405 & 121.550 & 2680 & 136.704 & 575 & 155.088 & 548 & 206.201 & 4740 \\
\hline 108.472 & 886 & 121.650 & 580 & 136.951 & 1906 & 156.098 & 422 & 207.036 & 301 \\
\hline 108.559 & 476 & 121.897 & 1999 & 137.049 & 3540 & 157.102 & 1357 & 207.207 & 834 \\
\hline 108.861 & 746 & 122.110 & 56997 & 137.099 & 3601 & 158.112 & 551 & 213.168 & 878 \\
\hline 108.905 & 414 & 122.554 & 1121 & 137.135 & 401872 & 159.120 & 7999 & 215.182 & 5352 \\
\hline 109.104 & 176043 & 122.606 & 1086 & 137.519 & 2007 & 159.212 & 390 & 216.189 & 3052 \\
\hline 109.449 & 991 & 122.658 & 716 & 137.558 & 2210 & 160.126 & 2648 & 217.197 & 15556 \\
\hline 109.482 & 1289 & 122.933 & 800 & 137.594 & 3002 & 161.135 & 38604 & 217.318 & 548 \\
\hline 109.519 & 2126 & 122.997 & 978 & 137.644 & 3250 & 161.237 & 1153 & 218.204 & 10181 \\
\hline 109.841 & 1436 & 123.089 & 727 & 137.683 & 995 & 162.142 & 15561 & 219.210 & 3406 \\
\hline 109.894 & 1397 & 123.120 & 197386 & 137.714 & 960 & 163.151 & 35655 & 220.218 & 595 \\
\hline 109.943 & 412 & 123.482 & 1331 & 137.997 & 2955 & 164.155 & 5988 & 227.184 & 584 \\
\hline 110.109 & 20718 & 123.520 & 1076 & 138.139 & 43037 & 165.074 & 483 & 228.195 & 409 \\
\hline 110.193 & 1827 & 123.564 & 2070 & 138.241 & 1562 & 165.165 & 2224 & 229.197 & 5872 \\
\hline 110.493 & 698 & 123.669 & 601 & 138.310 & 2115 & 166.172 & 288 & 230.204 & 2269 \\
\hline 110.549 & 943 & 123.890 & 873 & 138.364 & 2065 & 169.112 & 400 & 231.213 & 18283 \\
\hline 110.810 & 491 & 123.935 & 1492 & 138.711 & 929 & 171.119 & 989 & 231.317 & 583 \\
\hline 110.878 & 758 & 124.124 & 23353 & 138.747 & 838 & 172.126 & 308 & 232.216 & 3915 \\
\hline 110.947 & 445 & 124.223 & 1138 & 138.791 & 763 & 173.135 & 6270 & 233.227 & 2219 \\
\hline 111.084 & 1225 & 124.281 & 1172 & 139.143 & 3627 & 174.142 & 2194 & 234.232 & 468 \\
\hline 111.120 & 22687 & 124.349 & 508 & 139.454 & 504 & 175.151 & 38566 & 234.941 & 262 \\
\hline 111.175 & 619 & 124.601 & 483 & 139.501 & 476 & 175.263 & 1356 & 241.198 & 611 \\
\hline 111.207 & 1218 & 124.667 & 604 & 139.879 & 331 & 176.070 & 478 & 243.213 & 3492 \\
\hline 111.251 & 796 & 125.049 & 488 & 141.075 & 948 & 176.157 & 12184 & 244.218 & 1452 \\
\hline 111.326 & 265 & 125.134 & 6491 & 142.080 & 718 & 177.166 & 26900 & 245.228 & 2832 \\
\hline 111.521 & 437 & 125.336 & 405 & 143.088 & 1342 & 178.081 & 362 & 246.236 & 1190 \\
\hline 111.549 & 340 & 125.775 & 304 & 144.095 & 822 & 178.172 & 6034 & 247.238 & 496 \\
\hline 111.588 & 484 & 126.138 & 1042 & 145.104 & 7515 & 179.182 & 2781 & 249.945 & 310 \\
\hline 111.899 & 459 & 127.059 & 801 & 146.110 & 3073 & 180.185 & 565 & 251.945 & 651 \\
\hline 112.091 & 560 & 127.156 & 349 & 147.120 & 50129 & 185.139 & 1158 & 255.217 & 441 \\
\hline 112.124 & 2536 & 128.065 & 1795 & 147.560 & 402 & 186.139 & 441 & 257.228 & 5576 \\
\hline 112.177 & 421 & 129.072 & 2870 & 147.608 & 547 & 187.151 & 5953 & 258.234 & 2151 \\
\hline 112.223 & 444 & 130.081 & 1041 & 148.126 & 20767 & 188.157 & 2285 & 259.243 & 5789 \\
\hline 113.098 & 369 & 131.089 & 4982 & 148.208 & 446 & 189.166 & 34319 & 260.250 & 2736 \\
\hline 113.134 & 601 & 132.096 & 3590 & 148.982 & 414 & 190.172 & 11452 & 261.257 & 1056 \\
\hline
\end{tabular}


Table S19 (continued). High-resolution mass table for the compound identified as squalene.

\begin{tabular}{|c|c|c|c|c|c|c|c|c|c|}
\hline Mass & $\begin{array}{c}\text { Ion } \\
\text { counts }\end{array}$ & Mass & $\begin{array}{c}\text { Ion } \\
\text { counts }\end{array}$ & Mass & $\begin{array}{c}\text { Ion } \\
\text { counts }\end{array}$ & Mass & $\begin{array}{c}\text { Ion } \\
\text { counts }\end{array}$ & Mass & $\begin{array}{c}\text { Ion } \\
\text { counts }\end{array}$ \\
\hline 269.230 & 700 & 285.260 & 3190 & 313.289 & 1233 & 341.321 & 33663 & 369.344 & 708 \\
\hline 271.244 & 4132 & 286.264 & 2194 & 314.294 & 512 & 341.422 & 651 & 395.366 & 3408 \\
\hline 272.249 & 2025 & 287.274 & 744 & 325.289 & 2968 & 341.475 & 1342 & 396.371 & 1284 \\
\hline 273.259 & 13008 & 297.259 & 2066 & 326.297 & 1537 & 342.324 & 9138 & 397.174 & 834 \\
\hline 273.384 & 437 & 298.263 & 841 & 327.305 & 3417 & 343.325 & 1488 & 410.390 & 8068 \\
\hline 274.263 & 3674 & 299.275 & 9629 & 328.312 & 4483 & 353.319 & 498 & 411.395 & 2748 \\
\hline 275.266 & 578 & 300.278 & 2594 & 329.318 & 1130 & 354.328 & 442 & & \\
\hline 283.241 & 724 & 301.290 & 1441 & 339.308 & 1294 & 367.336 & 14568 & & \\
\hline 284.247 & 386 & 311.277 & 417 & 340.318 & 575 & 368.339 & 5069 & & \\
\hline
\end{tabular}


Table S20. High-resolution mass table for the compound identified as cholest-3,5-diene.

\begin{tabular}{|c|c|c|c|c|c|c|c|c|c|}
\hline Mass & $\begin{array}{c}\text { Ion } \\
\text { counts }\end{array}$ & Mass & $\begin{array}{c}\text { Ion } \\
\text { counts }\end{array}$ & Mass & $\begin{array}{c}\text { Ion } \\
\text { counts }\end{array}$ & Mass & $\begin{array}{c}\text { Ion } \\
\text { counts }\end{array}$ & Mass & $\begin{array}{c}\text { Ion } \\
\text { counts }\end{array}$ \\
\hline 50.018 & 890 & 79.128 & 882 & \begin{tabular}{|l|}
105.433 \\
\end{tabular} & 565 & 129.156 & 454 & 160.126 & 13724 \\
\hline 51.026 & 2250 & 79.349 & 469 & \begin{tabular}{|l|}
105.470 \\
\end{tabular} & 352 & 130.079 & 7215 & 161.134 & 16638 \\
\hline 52.034 & 1022 & 79.379 & 259 & 105.502 & 335 & 131.088 & 23924 & 161.251 & 480 \\
\hline 53.041 & 9894 & 80.063 & 7030 & \begin{tabular}{|l|l|}
105.537 \\
\end{tabular} & 463 & 131.180 & 649 & 162.141 & 4787 \\
\hline 54.049 & 3383 & 81.037 & 431 & \begin{tabular}{|l|l|}
105.778 \\
\end{tabular} & 458 & 132.094 & 6386 & 163.150 & 9474 \\
\hline 55.021 & 2209 & 81.073 & 97947 & 105.819 & 432 & 133.103 & 25445 & 164.155 & 1455 \\
\hline 55.057 & 51030 & 81.398 & 864 & \begin{tabular}{|l|}
106.079 \\
\end{tabular} & 16743 & 133.183 & 589 & 165.072 & 1335 \\
\hline 55.325 & 447 & 81.698 & 517 & \begin{tabular}{|l|}
106.160 \\
\end{tabular} & 700 & 134.110 & 8283 & 165.166 & 2174 \\
\hline 55.385 & 391 & 81.758 & 462 & \begin{tabular}{|l|}
107.088 \\
\end{tabular} & 49287 & 135.119 & 25791 & 166.080 & 548 \\
\hline 56.029 & 435 & 81.995 & 400 & 107.461 & 529 & 135.211 & 590 & 166.170 & 496 \\
\hline 56.064 & 7261 & 82.037 & 714 & \begin{tabular}{|l|}
107.497 \\
\end{tabular} & 468 & 136.080 & 565 & 167.090 & 1166 \\
\hline 57.036 & 1856 & 82.078 & 11130 & 107.535 & 463 & 136.126 & 8457 & 168.096 & 699 \\
\hline 57.073 & 39491 & 83.052 & 1181 & 107.797 & 432 & 137.134 & 11301 & 169.103 & 2170 \\
\hline 57.134 & 896 & 83.088 & 18874 & 108.095 & 17524 & 138.138 & 1705 & 170.109 & 1080 \\
\hline 57.165 & 448 & 83.166 & 479 & 108.174 & 672 & 139.059 & 431 & 171.119 & 7098 \\
\hline 57.351 & 351 & 83.216 & 396 & \begin{tabular}{|l|}
109.070 \\
\end{tabular} & 428 & 139.151 & 886 & 172.126 & 3423 \\
\hline 58.044 & 847 & 84.057 & 2044 & 109.104 & 26052 & 141.073 & 5069 & 173.134 & 8144 \\
\hline 58.076 & 2465 & 84.093 & 2463 & \begin{tabular}{|l|}
109.188 \\
\end{tabular} & 744 & 142.080 & 4809 & 174.140 & 3269 \\
\hline 59.052 & 1061 & 85.060 & 1368 & 109.225 & 448 & 143.088 & 27789 & 175.150 & 6106 \\
\hline 60.023 & 3262 & 85.104 & 5658 & 110.108 & 3458 & 143.173 & 695 & 176.157 & 1781 \\
\hline 61.031 & 1476 & 86.108 & 453 & 111.083 & 1215 & 143.209 & 488 & 177.165 & 3549 \\
\hline 63.026 & 805 & 87.048 & 1220 & 111.119 & 7004 & 144.094 & 8966 & 178.080 & 476 \\
\hline 64.035 & 249 & 89.054 & 618 & 112.091 & 806 & 145.103 & 52796 & 178.171 & 820 \\
\hline 65.042 & 5162 & 90.048 & 350 & 112.125 & 831 & 145.504 & 435 & 179.087 & 579 \\
\hline 66.049 & 2261 & 91.057 & 49581 & 113.097 & 391 & 145.547 & 331 & 179.182 & 1746 \\
\hline 67.057 & 40357 & 91.136 & 1431 & 113.135 & 973 & 145.578 & 202 & 180.094 & 379 \\
\hline 68.064 & 12578 & 91.355 & 397 & 114.058 & 349 & 145.621 & 459 & 180.186 & 434 \\
\hline 69.037 & 512 & 91.429 & 463 & 115.057 & 8387 & 146.110 & 16757 & 181.103 & 508 \\
\hline 69.073 & 97151 & 92.064 & 12861 & \begin{tabular}{|l|}
116.064 \\
\end{tabular} & 4350 & 147.119 & 67448 & 182.112 & 420 \\
\hline 69.373 & 696 & 92.128 & 426 & 117.072 & 19087 & 147.559 & 579 & 183.119 & 1910 \\
\hline 69.412 & 765 & 93.073 & 42368 & 117.161 & 456 & 147.610 & 630 & 184.126 & 1084 \\
\hline 69.634 & 408 & 93.149 & 984 & \begin{tabular}{|l|}
118.079 \\
\end{tabular} & 7865 & 147.961 & 398 & 185.135 & 7477 \\
\hline 69.678 & 469 & 94.079 & 11721 & \begin{tabular}{|l|}
119.088 \\
\end{tabular} & 26105 & 148.014 & 473 & 186.141 & 2876 \\
\hline 69.897 & 379 & 95.051 & 620 & 119.145 & 268 & 148.124 & 14191 & 187.150 & 3702 \\
\hline 69.963 & 442 & 95.088 & 46519 & \begin{tabular}{|l|l|}
119.175 \\
\end{tabular} & 540 & 148.223 & 370 & 188.156 & 1023 \\
\hline 70.044 & 665 & 95.478 & 453 & 119.890 & 381 & 149.026 & 2404 & 189.166 & 2576 \\
\hline 70.077 & 7926 & 95.773 & 393 & 120.095 & 28593 & 149.134 & 16936 & 190.171 & 773 \\
\hline 70.159 & 426 & 96.063 & 680 & 120.518 & 440 & 149.225 & 530 & 191.181 & 3828 \\
\hline 71.051 & 928 & 96.093 & 5776 & 121.103 & 32481 & 150.038 & 368 & 192.186 & 1471 \\
\hline 71.088 & 19695 & 97.067 & 1310 & \begin{tabular}{|l|l|}
121.193 \\
\end{tabular} & 1008 & 150.139 & 2700 & 193.197 & 3463 \\
\hline 71.145 & 373 & 97.104 & 11405 & 122.110 & 8253 & 151.150 & 3029 & 194.197 & 625 \\
\hline 72.059 & 338 & 98.075 & 2913 & \begin{tabular}{|l|l|}
123.119 \\
\end{tabular} & 13944 & 152.065 & 846 & 195.122 & 375 \\
\hline 72.091 & 1171 & 98.109 & 1441 & 124.124 & 2332 & 152.157 & 665 & 197.134 & 2261 \\
\hline 73.031 & 3869 & 99.080 & 560 & 125.095 & 515 & 153.073 & 1345 & 198.141 & 1172 \\
\hline 73.065 & 446 & 99.119 & 1027 & 125.134 & 2007 & 153.165 & 558 & 199.150 & 8109 \\
\hline 74.039 & 435 & 100.070 & 369 & 126.055 & 374 & 154.080 & 1324 & 200.156 & 3846 \\
\hline 75.028 & 812 & 101.061 & 578 & 126.140 & 404 & 155.088 & 3047 & 201.165 & 5063 \\
\hline 76.034 & 363 & 102.049 & 644 & 127.057 & 2453 & 156.094 & 1733 & 202.170 & 1157 \\
\hline 77.041 & 15558 & 103.057 & 4483 & 127.150 & 985 & 157.103 & 11445 & 203.181 & 1847 \\
\hline 77.111 & 386 & 104.065 & 4467 & 128.065 & 9658 & 158.110 & 9868 & 204.190 & 763 \\
\hline 78.048 & 5678 & 105.073 & 58145 & \begin{tabular}{|l|l|}
128.154 \\
\end{tabular} & 385 & 159.119 & 21379 & 205.197 & 5613 \\
\hline 79.057 & 36538 & 105.156 & 1719 & 129.072 & 15418 & 159.220 & 517 & 206.203 & 4018 \\
\hline
\end{tabular}


Table S20 (continued). High-resolution mass table for the compound identified as cholest-3,5-diene.

\begin{tabular}{|c|c|c|c|c|c|c|c|c|c|}
\hline Mass & $\begin{array}{c}\text { Ion } \\
\text { counts }\end{array}$ & Mass & $\begin{array}{c}\text { Ion } \\
\text { counts }\end{array}$ & Mass & $\begin{array}{c}\text { Ion } \\
\text { counts }\end{array}$ & Mass & $\begin{array}{c}\text { Ion } \\
\text { counts }\end{array}$ & Mass & $\begin{array}{c}\text { Ion } \\
\text { counts }\end{array}$ \\
\hline 207.212 & 1791 & 228.188 & 4475 & 249.253 & 899 & 269.232 & 523 & 326.296 & 3886 \\
\hline 208.216 & 334 & 229.195 & 2962 & 249.940 & 283 & 271.241 & 525 & 327.303 & 1283 \\
\hline 211.151 & 1221 & 230.207 & 590 & 251.946 & 412 & 273.257 & 1167 & 328.302 & 285 \\
\hline 212.157 & 1219 & 231.214 & 1481 & 253.196 & 977 & 274.266 & 1955 & 339.304 & 932 \\
\hline 213.165 & 24308 & 232.218 & 668 & 254.205 & 402 & 275.270 & 1138 & 340.311 & 929 \\
\hline 213.281 & 776 & 233.227 & 827 & 255.211 & 18828 & 283.243 & 2570 & 341.319 & 817 \\
\hline 214.170 & 6474 & 239.180 & 1067 & 255.334 & 624 & 284.247 & 791 & 353.319 & 30590 \\
\hline 215.180 & 3107 & 240.185 & 567 & 256.214 & 4339 & 285.256 & 502 & 353.473 & 1142 \\
\hline 216.186 & 1013 & 241.196 & 2269 & 257.226 & 1226 & 287.280 & 296 & 354.322 & 8853 \\
\hline 217.197 & 1063 & 242.202 & 619 & 258.233 & 834 & 297.260 & 765 & 355.327 & 1619 \\
\hline 218.205 & 743 & 243.213 & 480 & 259.242 & 3461 & 298.261 & 457 & 366.325 & 1547 \\
\hline 219.213 & 1876 & 245.227 & 4848 & 260.250 & 15452 & 299.274 & 574 & 367.333 & 1131 \\
\hline 220.219 & 472 & 246.234 & 3427 & 260.374 & 622 & 311.273 & 808 & 368.342 & 45920 \\
\hline 225.166 & 786 & 247.243 & 24199 & 261.256 & 5426 & 313.292 & 348 & 368.488 & 2153 \\
\hline 226.174 & 555 & 247.376 & 856 & 262.261 & 1325 & 314.298 & 881 & 369.345 & 13832 \\
\hline 227.180 & 3248 & 248.247 & 5771 & 267.209 & 391 & 325.300 & 962 & 370.348 & 2313 \\
\hline
\end{tabular}


Table S21. High-resolution mass table for the compound identified as 1,6,10,14,18,22-tetracoshexaen-3-ol 2,4,6,10,15,19,23-hexamethyl.

\begin{tabular}{|c|c|c|c|c|c|c|c|c|c|}
\hline Mass & $\begin{array}{c}\text { Ion } \\
\text { counts }\end{array}$ & Mass & $\begin{array}{c}\text { Ion } \\
\text { counts }\end{array}$ & Mass & $\begin{array}{c}\text { Ion } \\
\text { counts }\end{array}$ & Mass & $\begin{array}{c}\text { Ion } \\
\text { counts }\end{array}$ & Mass & $\begin{array}{c}\text { Ion } \\
\text { counts }\end{array}$ \\
\hline 50.018 & 1891 & 68.065 & 87590 & 73.031 & 3335 & 83.733 & 445 & 97.481 & 359 \\
\hline 51.026 & 4162 & 68.363 & 519 & 73.067 & 913 & 83.773 & 460 & 98.075 & 9427 \\
\hline 52.033 & 2591 & 68.396 & 709 & 74.018 & 334 & 84.060 & 14532 & 98.109 & 2121 \\
\hline 53.041 & 45307 & 68.420 & 799 & 74.037 & 399 & 84.093 & 4163 & 98.171 & 312 \\
\hline 53.316 & 389 & 68.476 & 443 & 75.028 & 730 & 85.067 & 6722 & 99.082 & 3019 \\
\hline 54.048 & 7845 & 68.641 & 784 & 76.033 & 354 & 85.104 & 3799 & 99.119 & 744 \\
\hline 55.021 & 8919 & 68.671 & 864 & 77.042 & 27267 & 86.073 & 498 & 100.053 & 360 \\
\hline 55.057 & 120645 & 68.699 & 659 & 77.114 & 540 & 86.107 & 253 & 101.062 & 805 \\
\hline 55.096 & 1157 & 68.755 & 508 & 78.048 & 6145 & 87.047 & 890 & 102.049 & 465 \\
\hline 55.301 & 523 & 68.831 & 359 & 79.057 & 67957 & 89.042 & 519 & 103.057 & 3235 \\
\hline 55.331 & 1148 & 68.924 & 1403 & 79.336 & 1681 & 90.046 & 265 & 104.065 & 1644 \\
\hline 55.360 & 899 & 68.963 & 1602 & 79.379 & 536 & 91.057 & 46252 & 105.073 & 44995 \\
\hline 55.551 & 462 & 69.036 & 12690 & 79.403 & 839 & 91.336 & 612 & 105.458 & 433 \\
\hline 55.607 & 729 & 69.074 & 884413 & 79.468 & 461 & 91.414 & 462 & 105.815 & 435 \\
\hline 55.817 & 562 & 69.118 & 72632 & 79.640 & 385 & 91.453 & 411 & 106.079 & 11160 \\
\hline 55.858 & 438 & 69.373 & 10999 & 79.670 & 523 & 92.064 & 20544 & 106.122 & 325 \\
\hline 56.027 & 1341 & 69.414 & 11353 & 79.708 & 495 & 93.073 & 145871 & 106.175 & 411 \\
\hline 56.063 & 11057 & 69.460 & 6115 & 79.976 & 425 & 93.387 & 855 & 107.053 & 1188 \\
\hline 56.123 & 465 & 69.485 & 2269 & 80.064 & 25811 & 93.422 & 736 & 107.088 & 100317 \\
\hline 56.310 & 392 & 69.665 & 8993 & 80.139 & 513 & 93.452 & 1281 & 107.431 & 389 \\
\hline 57.036 & 8614 & 69.928 & 6504 & 80.715 & 401 & 93.533 & 431 & 107.463 & 452 \\
\hline 57.073 & 32965 & 70.043 & 2670 & 80.798 & 467 & 93.711 & 557 & 107.502 & 885 \\
\hline 57.133 & 743 & 70.077 & 72229 & 80.829 & 318 & 93.743 & 445 & 107.572 & 419 \\
\hline 57.372 & 436 & 70.146 & 4089 & 80.863 & 401 & 93.768 & 700 & 107.823 & 602 \\
\hline 57.581 & 389 & 70.168 & 1679 & 80.950 & 670 & 93.815 & 522 & 107.877 & 460 \\
\hline 58.043 & 1445 & 70.185 & 2925 & 81.038 & 1872 & 94.044 & 934 & 108.063 & 1120 \\
\hline 58.076 & 1696 & 70.208 & 3943 & 81.073 & 505292 & 94.079 & 37872 & 108.095 & 23588 \\
\hline 59.052 & 8870 & 70.238 & 4245 & 81.120 & 4443 & 94.409 & 660 & 108.485 & 580 \\
\hline 60.024 & 2558 & 70.466 & 3500 & 81.369 & 3062 & 94.456 & 553 & 108.529 & 549 \\
\hline 60.055 & 351 & 70.500 & 3308 & 81.398 & 2684 & 94.716 & 495 & 108.889 & 459 \\
\hline 61.031 & 1116 & 70.719 & 2167 & 81.429 & 4705 & 94.996 & 402 & 109.068 & 9231 \\
\hline 62.018 & 386 & 70.748 & 2518 & 81.496 & 957 & 95.052 & 4977 & 109.104 & 121545 \\
\hline 63.026 & 1410 & 70.845 & 424 & 81.518 & 1136 & 95.088 & 162831 & 109.187 & 4187 \\
\hline 64.032 & 380 & 71.018 & 1783 & 81.712 & 4029 & 95.140 & 1793 & 109.427 & 396 \\
\hline 65.042 & 11201 & 71.052 & 24006 & 81.773 & 2330 & 95.409 & 909 & 109.483 & 1437 \\
\hline 66.049 & 5183 & 71.088 & 13335 & 82.010 & 3025 & 95.441 & 992 & 109.519 & 894 \\
\hline 67.057 & 111092 & 71.123 & 727 & 82.044 & 3332 & 95.474 & 1582 & 109.554 & 1358 \\
\hline 67.328 & 736 & 71.276 & 1242 & 82.078 & 70768 & 95.732 & 574 & 109.800 & 511 \\
\hline 67.352 & 839 & 71.313 & 1313 & 82.330 & 1921 & 95.782 & 895 & 109.859 & 943 \\
\hline 67.374 & 832 & 71.378 & 384 & 82.368 & 1485 & 95.853 & 445 & 109.907 & 619 \\
\hline 67.415 & 527 & 71.530 & 347 & 82.407 & 739 & 96.060 & 4680 & 110.075 & 6523 \\
\hline 67.593 & 433 & 71.556 & 437 & 82.587 & 983 & 96.094 & 20736 & 110.109 & 15590 \\
\hline 67.638 & 820 & 71.603 & 613 & 82.626 & 875 & 96.151 & 1164 & 110.165 & 957 \\
\hline 67.666 & 797 & 71.852 & 487 & 82.656 & 889 & 96.398 & 513 & 110.204 & 1421 \\
\hline 67.730 & 380 & 71.886 & 482 & 82.892 & 536 & 96.448 & 563 & 110.523 & 712 \\
\hline 67.846 & 415 & 72.058 & 3102 & 82.937 & 493 & 96.737 & 518 & 110.844 & 529 \\
\hline 67.884 & 692 & 72.092 & 885 & 83.052 & 5427 & 97.067 & 19074 & 110.896 & 488 \\
\hline 67.931 & 692 & 72.125 & 440 & 83.088 & 45313 & 97.104 & 18299 & 111.083 & 12530 \\
\hline 67.972 & 306 & 72.383 & 418 & 83.459 & 568 & 97.148 & 669 & 111.120 & 17851 \\
\hline 67.996 & 405 & 72.423 & 411 & 83.494 & 596 & 97.183 & 600 & 111.174 & 714 \\
\hline
\end{tabular}


Table S21 (continued). High-resolution mass table for the compound identified as 1,6,10,14,18,22-tetracoshexaen3-ol 2,4,6,10,15,19,23-hexamethyl.

\begin{tabular}{|c|c|c|c|c|c|c|c|c|c|}
\hline Mass & $\begin{array}{c}\text { Ion } \\
\text { counts }\end{array}$ & Mass & $\begin{array}{c}\text { Ion } \\
\text { counts }\end{array}$ & Mass & $\begin{array}{c}\text { Ion } \\
\text { counts }\end{array}$ & Mass & $\begin{array}{c}\text { Ion } \\
\text { counts }\end{array}$ & Mass & $\begin{array}{c}\text { Ion } \\
\text { counts }\end{array}$ \\
\hline 111.203 & 1108 & 127.066 & 1081 & 150.141 & 11865 & 188.156 & 2680 & 243.211 & 1673 \\
\hline 111.466 & 317 & 127.114 & 7987 & 151.114 & 8299 & 189.166 & 15764 & 244.218 & 489 \\
\hline 111.493 & 297 & 128.065 & 2079 & \begin{tabular}{|l|l|}
151.150 \\
\end{tabular} & 5003 & 189.266 & 504 & 245.227 & 1248 \\
\hline 111.562 & 411 & 128.118 & 823 & 151.232 & 451 & 190.172 & 6019 & 246.224 & 410 \\
\hline 111.907 & 461 & 129.072 & 3846 & 152.070 & 522 & 191.147 & 2012 & 247.206 & 1476 \\
\hline 112.090 & 2583 & 130.080 & 1437 & 152.121 & 5425 & 191.181 & 9517 & 247.240 & 969 \\
\hline 112.124 & 1791 & 131.088 & 7612 & \begin{tabular}{|l|l|}
152.152 \\
\end{tabular} & 939 & 192.152 & 1822 & 248.215 & 466 \\
\hline 113.099 & 2591 & 132.095 & 3052 & \begin{tabular}{|l|}
153.072 \\
\end{tabular} & 465 & 192.187 & 3979 & 251.946 & 479 \\
\hline 113.136 & 583 & 133.104 & 35074 & 153.130 & 8909 & 193.160 & 2723 & 255.213 & 1844 \\
\hline 114.076 & 388 & 134.111 & 17868 & \begin{tabular}{|l|l|}
154.075 \\
\end{tabular} & 461 & 193.194 & 1354 & 256.220 & 462 \\
\hline 115.057 & 3519 & 134.196 & 445 & 154.135 & 1665 & 194.167 & 1355 & 257.227 & 3764 \\
\hline 116.063 & 1644 & 135.083 & 2983 & 155.091 & 799 & 195.174 & 658 & 258.234 & 2040 \\
\hline 117.073 & 6157 & 135.119 & 92153 & 156.093 & 410 & 197.135 & 232 & 259.241 & 1631 \\
\hline 118.080 & 2164 & 135.500 & 464 & \begin{tabular}{|l|l|}
157.104 \\
\end{tabular} & 2558 & 199.149 & 1923 & 260.250 & 789 \\
\hline 119.088 & 44942 & 135.540 & 274 & 158.109 & 1262 & 200.154 & 668 & 261.224 & 1128 \\
\hline 119.176 & 1443 & 135.575 & 739 & 159.119 & 11376 & 201.165 & 6707 & 262.227 & 415 \\
\hline 119.478 & 395 & 135.634 & 509 & 160.126 & 3578 & 202.172 & 2684 & 269.226 & 1299 \\
\hline 119.528 & 396 & 135.947 & 497 & 161.135 & 22931 & 203.181 & 28428 & 270.236 & 564 \\
\hline 119.560 & 411 & 136.026 & 463 & 161.238 & 769 & 203.307 & 859 & 271.243 & 3992 \\
\hline 120.095 & 12358 & 136.090 & 1561 & 162.140 & 5962 & 204.186 & 7814 & 272.248 & 1292 \\
\hline 121.068 & 1905 & 136.126 & 57520 & 163.115 & 3324 & 204.287 & 442 & 273.256 & 2311 \\
\hline 121.104 & 105309 & 137.044 & 1065 & 163.150 & 13969 & 205.161 & 1934 & 274.244 & 705 \\
\hline 121.464 & 511 & \begin{tabular}{|c|}
137.099 \\
\end{tabular} & 13129 & 163.256 & 555 & 205.196 & 5731 & 275.239 & 1469 \\
\hline 121.503 & 1084 & 137.135 & 78052 & \begin{tabular}{|l|}
164.121 \\
\end{tabular} & 1418 & 206.167 & 1309 & 276.246 & 2942 \\
\hline 121.562 & 1001 & \begin{tabular}{|l|}
137.198 \\
\end{tabular} & 1334 & 164.155 & 2861 & 206.201 & 1882 & 277.249 & 772 \\
\hline 121.850 & 564 & 137.519 & 567 & 165.075 & 465 & 207.038 & 415 & 283.243 & 1256 \\
\hline 121.901 & 747 & 137.557 & 483 & \begin{tabular}{|l|}
165.130 \\
\end{tabular} & 7237 & 207.176 & 3968 & 284.251 & 432 \\
\hline 121.960 & 588 & \begin{tabular}{|l|}
137.598 \\
\end{tabular} & 326 & 165.165 & 1238 & 208.184 & 4611 & 285.260 & 625 \\
\hline 122.074 & 913 & 137.633 & 521 & 166.135 & 2064 & 209.190 & 1142 & 286.261 & 629 \\
\hline 122.110 & 29120 & 138.033 & 509 & \begin{tabular}{|l|}
166.171 \\
\end{tabular} & 835 & 211.145 & 257 & 287.249 & 839 \\
\hline 122.554 & 463 & 138.106 & 6509 & \begin{tabular}{|l|}
167.144 \\
\end{tabular} & 946 & 213.167 & 2002 & 289.253 & 1976 \\
\hline 122.604 & 502 & \begin{tabular}{|l|}
138.139 \\
\end{tabular} & 9817 & 169.104 & 591 & 214.171 & 762 & 290.257 & 528 \\
\hline 122.960 & 499 & 139.114 & 12712 & \begin{tabular}{|l|}
170.114 \\
\end{tabular} & 386 & 215.181 & 5595 & 297.258 & 1237 \\
\hline 123.083 & 8518 & \begin{tabular}{|l|}
139.148 \\
\end{tabular} & 993 & \begin{tabular}{|l|}
171.120 \\
\end{tabular} & 2213 & 216.188 & 2688 & 298.269 & 307 \\
\hline 123.119 & 83581 & 140.122 & 7102 & \begin{tabular}{|l|}
172.125 \\
\end{tabular} & 737 & 217.196 & 4325 & 299.275 & 1257 \\
\hline 123.492 & 532 & 141.073 & 1393 & \begin{tabular}{|l|}
173.135 \\
\end{tabular} & 6860 & 218.203 & 2475 & 300.275 & 453 \\
\hline 123.519 & 532 & \begin{tabular}{|l|}
141.126 \\
\end{tabular} & 1080 & \begin{tabular}{|l|}
174.141 \\
\end{tabular} & 2239 & 219.177 & 1526 & 301.253 & 754 \\
\hline 123.565 & 662 & \begin{tabular}{|l|}
142.079 \\
\end{tabular} & 956 & \begin{tabular}{|l|}
175.150 \\
\end{tabular} & 14957 & 219.208 & 1522 & 302.264 & 363 \\
\hline 123.648 & 504 & 143.088 & 2930 & \begin{tabular}{|l|}
175.255 \\
\end{tabular} & 482 & 220.187 & 1359 & 303.260 & 323 \\
\hline 123.925 & 589 & \begin{tabular}{|l|}
144.094 \\
\end{tabular} & 1483 & \begin{tabular}{|l|}
176.156 \\
\end{tabular} & 5543 & 221.191 & 4899 & 311.279 & 399 \\
\hline 124.091 & 5416 & 145.104 & 12246 & \begin{tabular}{|l|}
177.130 \\
\end{tabular} & 1480 & 222.197 & 795 & 313.255 & 483 \\
\hline 124.124 & 11540 & 146.110 & 3845 & 177.166 & 14322 & 227.183 & 1775 & 315.273 & 880 \\
\hline 124.207 & 611 & \begin{tabular}{|l|}
147.119 \\
\end{tabular} & 45544 & \begin{tabular}{|l|}
177.241 \\
\end{tabular} & 410 & 228.190 & 785 & 323.285 & 447 \\
\hline 124.258 & 381 & \begin{tabular}{|l|}
147.217 \\
\end{tabular} & 1701 & \begin{tabular}{|l|}
178.171 \\
\end{tabular} & 3672 & 229.197 & 4583 & 325.295 & 904 \\
\hline 124.301 & 503 & 147.580 & 466 & 179.145 & 2085 & 230.201 & 1127 & 326.298 & 624 \\
\hline 125.050 & 447 & 148.126 & 15118 & \begin{tabular}{|l|}
179.180 \\
\end{tabular} & 1868 & 231.212 & 4417 & 327.253 & 838 \\
\hline 125.099 & 13459 & \begin{tabular}{|l|}
148.223 \\
\end{tabular} & 492 & \begin{tabular}{|l|}
180.168 \\
\end{tabular} & 627 & 232.215 & 1122 & 327.300 & 635 \\
\hline 125.134 & 3309 & 149.098 & 2879 & 183.123 & 440 & 233.192 & 2324 & 328.309 & 412 \\
\hline 125.188 & 515 & 149.135 & 47196 & \begin{tabular}{|l|}
185.135 \\
\end{tabular} & 1939 & 234.198 & 862 & 329.303 & 531 \\
\hline 126.106 & 17486 & \begin{tabular}{|l|}
149.627 \\
\end{tabular} & 476 & \begin{tabular}{|l|}
186.141 \\
\end{tabular} & 657 & 235.205 & 488 & 337.287 & 329 \\
\hline 126.197 & 533 & 150.106 & 1763 & \begin{tabular}{|l|l|}
187.150 \\
\end{tabular} & 6886 & 241.199 & 1050 & 339.305 & 3719 \\
\hline
\end{tabular}


Table S21 (continued). High-resolution mass table for the compound identified as 1,6,10,14,18,22-tetracoshexaen3-ol 2,4,6,10,15,19,23-hexamethyl.

\begin{tabular}{|c|c|c|c|c|c|c|c|c|c|}
\hline Mass & $\begin{array}{c}\text { Ion } \\
\text { counts }\end{array}$ & Mass & $\begin{array}{c}\text { Ion } \\
\text { counts }\end{array}$ & Mass & $\begin{array}{c}\text { Ion } \\
\text { counts }\end{array}$ & Mass & $\begin{array}{c}\text { Ion } \\
\text { counts }\end{array}$ & Mass & $\begin{array}{c}\text { Ion } \\
\text { counts }\end{array}$ \\
\hline 340.309 & 1272 & 353.321 & 682 & 366.324 & 612 & 384.337 & 401 & 410.384 & 710 \\
\hline 341.319 & 2595 & 355.306 & 1011 & 367.336 & 1070 & 393.360 & 579 & 411.364 & 534 \\
\hline 342.322 & 649 & 357.316 & 4200 & 368.345 & 1171 & 398.385 & 417 & 426.385 & 1160 \\
\hline 343.306 & 415 & 358.318 & 1269 & 369.353 & 413 & 408.372 & 2395 & 427.388 & 466 \\
\hline 344.307 & 777 & 365.321 & 1153 & 383.330 & 1579 & 409.378 & 741 & &
\end{tabular}


Table S22. High-resolution mass table for the compound identified as tetradecanoic acid tetradecyl ester.

\begin{tabular}{|c|c|c|c|c|c|c|c|c|c|}
\hline Mass & $\begin{array}{c}\text { Ion } \\
\text { counts }\end{array}$ & Mass & $\begin{array}{c}\text { Ion } \\
\text { counts }\end{array}$ & Mass & $\begin{array}{c}\text { Ion } \\
\text { counts }\end{array}$ & Mass & $\begin{array}{c}\text { Ion } \\
\text { counts }\end{array}$ & Mass & $\begin{array}{c}\text { Ion } \\
\text { counts }\end{array}$ \\
\hline 50.018 & 521 & 63.029 & 342 & 83.727 & 442 & 105.073 & 1111 & 134.107 & 427 \\
\hline 51.025 & 835 & 65.042 & 1415 & 83.759 & 448 & 106.077 & 392 & 135.119 & 2621 \\
\hline 52.033 & 586 & 66.049 & 1932 & 84.060 & 10452 & 107.050 & 388 & 136.123 & 492 \\
\hline 53.041 & 4370 & 67.057 & 21619 & 84.095 & 36887 & 107.088 & 1940 & 137.046 & 414 \\
\hline 54.049 & 12283 & 67.127 & 467 & 84.140 & 444 & 108.096 & 919 & 137.098 & 501 \\
\hline 55.021 & 7657 & 68.028 & 269 & 84.168 & 774 & 109.069 & 348 & 137.134 & 2227 \\
\hline 55.057 & 110191 & 68.065 & 24785 & 84.398 & 366 & 109.104 & 7693 & 138.143 & 3293 \\
\hline 55.326 & 1035 & 68.130 & 627 & 85.066 & 2850 & 110.075 & 795 & 139.063 & 414 \\
\hline 55.354 & 826 & 69.036 & 1588 & 85.104 & 59026 & 110.111 & 8818 & 139.115 & 2707 \\
\hline 55.553 & 484 & 69.073 & 95715 & 85.438 & 287 & 111.083 & 5858 & 139.150 & 6185 \\
\hline 55.573 & 386 & 69.343 & 379 & 85.492 & 395 & 111.119 & 39664 & 140.118 & 555 \\
\hline 55.598 & 691 & 69.375 & 654 & 86.039 & 617 & 111.532 & 441 & 140.158 & 9082 \\
\hline 55.633 & 503 & 69.416 & 580 & 86.071 & 567 & 112.090 & 3447 & 141.080 & 407 \\
\hline 55.813 & 562 & 69.440 & 572 & 86.107 & 3921 & 112.126 & 16116 & 141.164 & 2443 \\
\hline 55.836 & 379 & 69.643 & 455 & 87.047 & 9914 & 112.228 & 614 & 143.109 & 5062 \\
\hline 55.872 & 424 & 69.680 & 518 & 87.083 & 843 & 112.585 & 436 & 144.114 & 674 \\
\hline 56.028 & 1446 & 69.929 & 415 & 87.120 & 412 & 113.063 & 651 & 145.123 & 580 \\
\hline 56.065 & 53829 & 70.044 & 1961 & 88.053 & 1342 & 113.098 & 701 & 147.118 & 312 \\
\hline 56.126 & 1592 & 70.080 & 52244 & 89.062 & 2718 & 113.134 & 6192 & 149.135 & 1637 \\
\hline 56.274 & 342 & 70.149 & 1537 & 91.057 & 2120 & 114.069 & 931 & 150.143 & 397 \\
\hline 56.309 & 550 & 70.388 & 325 & 92.065 & 534 & 114.137 & 614 & 151.113 & 645 \\
\hline 56.334 & 565 & 70.465 & 368 & 93.073 & 2931 & 115.078 & 8569 & 151.150 & 920 \\
\hline 56.356 & 478 & 71.052 & 3818 & 94.080 & 1482 & 116.085 & 6593 & 152.159 & 1652 \\
\hline 56.385 & 411 & 71.088 & 97549 & 95.052 & 497 & 116.159 & 311 & 153.129 & 1720 \\
\hline 56.563 & 514 & 71.359 & 384 & 95.088 & 15943 & 117.039 & 464 & 153.166 & 2834 \\
\hline 56.607 & 436 & 71.394 & 395 & 95.159 & 495 & 117.091 & 2117 & 154.174 & 3543 \\
\hline 57.036 & 4311 & 71.428 & 597 & 96.059 & 1502 & 119.088 & 807 & 155.180 & 1152 \\
\hline 57.073 & 182230 & 71.505 & 292 & 96.096 & 19178 & 120.092 & 320 & 157.125 & 4020 \\
\hline 57.319 & 1193 & 71.688 & 452 & 96.155 & 367 & 121.103 & 2624 & 158.131 & 704 \\
\hline 57.346 & 1199 & 72.059 & 683 & 97.068 & 8592 & 122.110 & 771 & 159.137 & 563 \\
\hline 57.376 & 1763 & 72.092 & 5229 & 97.104 & 74205 & 123.084 & 735 & 161.134 & 359 \\
\hline 57.609 & 1508 & 72.223 & 355 & 97.181 & 2479 & 123.119 & 3789 & 163.151 & 1162 \\
\hline 57.640 & 1304 & 73.031 & 33628 & 97.427 & 512 & 124.089 & 541 & 164.158 & 522 \\
\hline 57.679 & 449 & 73.068 & 2527 & 97.461 & 694 & 124.127 & 4630 & 165.080 & 345 \\
\hline 57.826 & 743 & 73.309 & 396 & 97.510 & 471 & 125.099 & 3569 & 165.167 & 774 \\
\hline 57.872 & 1039 & 74.038 & 3829 & 97.541 & 531 & 125.135 & 18253 & 166.174 & 2501 \\
\hline 58.044 & 2259 & 75.032 & 1120 & 97.746 & 388 & 125.191 & 273 & 167.145 & 836 \\
\hline 58.076 & 8211 & 77.041 & 1688 & 97.802 & 521 & 125.228 & 430 & 167.181 & 1890 \\
\hline 58.111 & 595 & 78.048 & 1335 & 98.075 & 13198 & 126.105 & 1014 & 168.189 & 15006 \\
\hline 58.141 & 511 & 79.057 & 4847 & 98.111 & 24476 & 126.142 & 10078 & 168.287 & 502 \\
\hline 58.331 & 486 & 80.064 & 2203 & 98.473 & 586 & 126.236 & 443 & 169.193 & 2994 \\
\hline 58.355 & 569 & 81.036 & 476 & 98.522 & 402 & 127.078 & 715 & 171.140 & 5347 \\
\hline 58.564 & 362 & 81.073 & 18880 & 98.763 & 391 & 127.114 & 445 & 172.145 & 997 \\
\hline 58.597 & 418 & 81.143 & 446 & 99.047 & 876 & 127.149 & 3651 & 173.156 & 1290 \\
\hline 59.052 & 2736 & 82.045 & 392 & 99.081 & 2143 & 128.064 & 460 & 177.168 & 473 \\
\hline 59.081 & 357 & 82.080 & 37508 & 99.119 & 10725 & 128.155 & 351 & 179.184 & 378 \\
\hline 60.023 & 20550 & 82.156 & 899 & 100.055 & 1098 & 129.093 & 20709 & 181.158 & 852 \\
\hline 60.073 & 405 & 83.052 & 6524 & 100.121 & 933 & 129.189 & 570 & 182.204 & 1023 \\
\hline 60.100 & 436 & 83.088 & 90353 & 101.062 & 7381 & 130.098 & 2847 & 183.176 & 2960 \\
\hline 61.031 & 26769 & 83.386 & 488 & 102.069 & 1865 & 131.053 & 357 & 184.180 & 431 \\
\hline 61.090 & 840 & 83.418 & 420 & 103.078 & 2990 & 131.107 & 1072 & 185.155 & 11578 \\
\hline 62.034 & 621 & 83.450 & 723 & 104.066 & 426 & 133.105 & 778 & 186.158 & 1601 \\
\hline
\end{tabular}


Table S22 (continued). High-resolution mass table for the compound identified as tetradecanoic acid tetradecyl ester.

\begin{tabular}{|c|c|c|c|c|c|c|c|c|c|}
\hline Mass & $\begin{array}{c}\text { Ion } \\
\text { counts }\end{array}$ & Mass & $\begin{array}{c}\text { Ion } \\
\text { counts }\end{array}$ & Mass & $\begin{array}{c}\text { Ion } \\
\text { counts }\end{array}$ & Mass & $\begin{array}{c}\text { Ion } \\
\text { counts }\end{array}$ & Mass & $\begin{array}{c}\text { Ion } \\
\text { counts }\end{array}$ \\
\hline 187.169 & 945 & 208.186 & 620 & 226.225 & 414 & 231.726 & 373 & 259.252 & 398 \\
\hline 191.180 & 1273 & 209.191 & 1482 & 227.202 & 1586 & 237.221 & 476 & 269.248 & 1652 \\
\hline 192.187 & 766 & 210.213 & 514 & 228.209 & 15689 & 239.238 & 4872 & 270.250 & 395 \\
\hline 193.197 & 745 & 211.207 & 20539 & 228.301 & 511 & 240.242 & 991 & 279.266 & 244 \\
\hline 194.205 & 827 & 211.330 & 603 & 229.217 & 111865 & 241.216 & 3628 & 283.264 & 398 \\
\hline 195.193 & 454 & 212.211 & 3373 & 229.781 & 1238 & 242.223 & 1179 & 284.268 & 573 \\
\hline 196.220 & 12027 & 213.187 & 4027 & 229.880 & 889 & 243.232 & 3458 & 297.278 & 339 \\
\hline 197.225 & 3440 & 214.191 & 984 & 230.220 & 17220 & 244.239 & 542 & 325.312 & 528 \\
\hline 199.171 & 3189 & 215.202 & 2842 & 230.298 & 1099 & 251.945 & 463 & 381.373 & 389 \\
\hline 200.178 & 2649 & 216.205 & 302 & 230.341 & 1334 & 255.230 & 692 & 424.425 & 2359 \\
\hline 201.186 & 14652 & 219.207 & 371 & 230.786 & 823 & 256.240 & 4158 & 425.427 & 863 \\
\hline 201.278 & 455 & 220.221 & 218 & 230.887 & 472 & 257.247 & 23174 & \\
\hline 202.189 & 2309 & 224.251 & 783 & 231.223 & 2179 & 257.362 & 744 & \\
\hline 207.037 & 269 & 225.222 & 957 & 231.321 & 603 & 258.252 & 4165 &
\end{tabular}


Table S23. High-resolution mass table for the compound identified as cholesterol.

\begin{tabular}{|c|c|c|c|c|c|c|c|c|c|}
\hline Mass & $\begin{array}{c}\text { Ion } \\
\text { counts }\end{array}$ & Mass & $\begin{array}{c}\text { Ion } \\
\text { counts }\end{array}$ & Mass & $\begin{array}{c}\text { Ion } \\
\text { counts }\end{array}$ & Mass & $\begin{array}{c}\text { Ion } \\
\text { counts }\end{array}$ & Mass & $\begin{array}{c}\text { Ion } \\
\text { counts }\end{array}$ \\
\hline 50.018 & 1151 & 65.103 & 381 & 77.042 & 49417 & 86.065 & 783 & 96.450 & 704 \\
\hline 51.026 & 2802 & 66.049 & 6343 & 77.322 & 514 & 86.107 & 1303 & 96.724 & 551 \\
\hline 52.033 & 1935 & 67.021 & 522 & 77.360 & 228 & 87.048 & 1029 & 96.758 & 429 \\
\hline 53.042 & 25512 & 67.057 & 122721 & 77.428 & 444 & 87.072 & 832 & 96.792 & 464 \\
\hline 53.092 & 487 & 67.320 & 585 & 77.674 & 343 & 89.043 & 1163 & 97.068 & 9142 \\
\hline 53.106 & 457 & 67.354 & 751 & 78.049 & 14196 & 90.049 & 749 & 97.104 & 48329 \\
\hline 54.049 & 6245 & 67.377 & 928 & 79.057 & 146361 & 91.057 & 163807 & 97.427 & 462 \\
\hline 55.021 & 11402 & 67.413 & 777 & 79.339 & 1372 & 91.341 & 911 & 97.461 & 297 \\
\hline 55.057 & 209672 & 67.648 & 794 & 79.389 & 1545 & 91.373 & 957 & 97.495 & 450 \\
\hline 55.300 & 1290 & 67.698 & 455 & 79.439 & 1185 & 91.403 & 1019 & 97.751 & 358 \\
\hline 55.325 & 1392 & 67.878 & 454 & 79.637 & 472 & 91.439 & 1640 & 97.804 & 380 \\
\hline 55.355 & 1961 & 67.923 & 443 & 79.684 & 1014 & 91.481 & 885 & 98.075 & 3403 \\
\hline 55.384 & 1248 & 67.950 & 268 & 79.727 & 860 & 91.529 & 307 & 98.108 & 4375 \\
\hline 55.424 & 329 & 68.064 & 19771 & 79.958 & 513 & 91.696 & 552 & 99.081 & 2054 \\
\hline 55.539 & 575 & 68.126 & 661 & 79.996 & 478 & 91.734 & 1051 & 99.120 & 3108 \\
\hline 55.589 & 1539 & 68.164 & 516 & 80.063 & 22703 & 91.991 & 351 & 100.081 & 1454 \\
\hline 55.622 & 1000 & 68.211 & 364 & 80.125 & 355 & 92.064 & 39556 & 100.124 & 333 \\
\hline 55.816 & 990 & 69.037 & 2790 & 80.267 & 425 & 92.140 & 1306 & 101.063 & 692 \\
\hline 55.841 & 1007 & 69.073 & 127812 & 80.567 & 472 & 92.329 & 496 & 102.049 & 1088 \\
\hline 55.880 & 479 & 69.348 & 444 & 80.855 & 411 & 92.372 & 717 & 103.057 & 11722 \\
\hline 56.027 & 1507 & 69.374 & 719 & 81.007 & 394 & 92.412 & 645 & 104.065 & 11687 \\
\hline 56.063 & 21720 & 69.411 & 1022 & 81.037 & 2155 & 92.678 & 440 & 105.041 & 1063 \\
\hline 56.120 & 1179 & 69.608 & 360 & 81.073 & 204332 & 92.962 & 418 & 105.073 & 197162 \\
\hline 56.333 & 622 & 69.654 & 693 & 81.370 & 1058 & 92.996 & 326 & 105.358 & 420 \\
\hline 56.576 & 393 & 69.695 & 618 & 81.399 & 1237 & 93.073 & 158837 & 105.409 & 1235 \\
\hline 57.037 & 17467 & 69.725 & 447 & 81.439 & 2237 & 93.388 & 773 & 105.444 & 1057 \\
\hline 57.073 & 160460 & 69.941 & 475 & 81.523 & 411 & 93.422 & 1002 & 105.479 & 2050 \\
\hline 57.113 & 1724 & 69.975 & 452 & 81.695 & 1676 & 93.457 & 1257 & 105.547 & 732 \\
\hline 57.324 & 1262 & 70.044 & 927 & 81.734 & 1737 & 93.575 & 646 & 105.579 & 501 \\
\hline 57.346 & 1080 & 70.078 & 12547 & 81.783 & 788 & 93.694 & 454 & 105.820 & 1753 \\
\hline 57.370 & 1576 & 70.218 & 394 & 81.933 & 298 & 93.763 & 707 & 106.079 & 55275 \\
\hline 57.425 & 408 & 70.465 & 395 & 81.970 & 847 & 93.791 & 651 & 106.450 & 1242 \\
\hline 57.583 & 791 & 70.725 & 375 & 82.010 & 1191 & 94.045 & 1356 & 106.493 & 1088 \\
\hline 57.609 & 1032 & 71.052 & 3623 & 82.043 & 2198 & 94.080 & 47286 & 106.532 & 935 \\
\hline 57.635 & 1130 & 71.089 & 79436 & 82.078 & 25674 & 94.150 & 1628 & 106.585 & 593 \\
\hline 57.805 & 456 & 71.365 & 380 & 82.291 & 575 & 94.385 & 428 & 106.773 & 578 \\
\hline 57.831 & 330 & 71.393 & 223 & 82.337 & 299 & 94.426 & 557 & 106.807 & 1003 \\
\hline 57.861 & 771 & 71.421 & 474 & 82.366 & 473 & 94.701 & 428 & 106.853 & 863 \\
\hline 58.042 & 2152 & 71.453 & 472 & 82.601 & 341 & 94.744 & 461 & 106.891 & 495 \\
\hline 58.076 & 7512 & 71.687 & 482 & 82.636 & 502 & 95.052 & 5123 & 107.053 & 3679 \\
\hline 58.130 & 592 & 71.718 & 411 & 83.052 & 9914 & 95.088 & 201025 & 107.088 & 219502 \\
\hline 58.319 & 432 & 71.979 & 383 & 83.088 & 80503 & 95.409 & 1108 & 107.424 & 1705 \\
\hline 58.350 & 520 & 72.019 & 294 & 83.392 & 386 & 95.441 & 1088 & 107.463 & 2260 \\
\hline 58.888 & 349 & 72.057 & 1244 & 83.417 & 475 & 95.473 & 1726 & 107.510 & 2581 \\
\hline 59.052 & 2350 & 72.092 & 4410 & 83.467 & 748 & 95.546 & 278 & 107.596 & 386 \\
\hline 59.085 & 377 & 73.031 & 3695 & 83.750 & 540 & 95.778 & 1204 & 107.844 & 1992 \\
\hline 60.024 & 3084 & 73.064 & 735 & 84.004 & 355 & 95.811 & 1182 & 108.060 & 2438 \\
\hline 61.031 & 1366 & 73.091 & 298 & 84.059 & 3520 & 96.060 & 4096 & 108.095 & 53529 \\
\hline 62.020 & 321 & 74.017 & 263 & 84.093 & 7110 & 96.093 & 22420 & 108.437 & 833 \\
\hline 63.025 & 1161 & 74.040 & 334 & 85.067 & 3953 & 96.166 & 1258 & 108.479 & 1419 \\
\hline 64.034 & 476 & 75.028 & 606 & 85.104 & 16230 & 96.376 & 475 & 108.533 & 1400 \\
\hline 65.042 & 14008 & 76.035 & 521 & 85.182 & 469 & 96.415 & 372 & 108.824 & 1123 \\
\hline
\end{tabular}


Table S23 (continued). High-resolution mass table for the compound identified as cholesterol.

\begin{tabular}{|c|c|c|c|c|c|c|c|c|c|}
\hline Mass & $\begin{array}{c}\text { Ion } \\
\text { counts }\end{array}$ & Mass & $\begin{array}{c}\text { Ion } \\
\text { counts }\end{array}$ & Mass & $\begin{array}{c}\text { Ion } \\
\text { counts }\end{array}$ & Mass & $\begin{array}{c}\text { Ion } \\
\text { counts }\end{array}$ & Mass & $\begin{array}{c}\text { Ion } \\
\text { counts }\end{array}$ \\
\hline 108.868 & 1186 & 120.096 & 101861 & 133.415 & 296 & 145.978 & 1252 & 159.590 & 1295 \\
\hline 109.068 & 9523 & 120.493 & 1049 & 133.486 & 634 & 146.031 & 760 & 160.039 & 832 \\
\hline 109.104 & 101129 & 120.532 & 1117 & 133.529 & 801 & 146.075 & 874 & 160.090 & 2785 \\
\hline 109.159 & 1434 & 120.570 & 1114 & 133.569 & 731 & 146.110 & 47898 & 160.126 & 73830 \\
\hline 109.179 & 2235 & 120.603 & 1054 & 133.605 & 814 & 146.686 & 491 & 160.232 & 2138 \\
\hline 109.199 & 3155 & 120.822 & 486 & 133.859 & 424 & 147.083 & 4180 & 160.377 & 356 \\
\hline 109.448 & 834 & 120.893 & 951 & 133.896 & 412 & 147.120 & 110782 & 160.544 & 483 \\
\hline 109.492 & 1521 & 120.956 & 761 & 133.942 & 731 & 147.213 & 3860 & 160.613 & 436 \\
\hline 109.530 & 1304 & 121.068 & 4731 & 134.017 & 411 & 147.512 & 617 & 161.037 & 490 \\
\hline 109.566 & 1105 & 121.104 & 114519 & 134.074 & 1449 & 147.559 & 685 & 161.099 & 13636 \\
\hline 109.858 & 1340 & 121.462 & 473 & 134.111 & 42716 & 147.590 & 840 & 161.135 & 109681 \\
\hline 109.893 & 927 & 121.519 & 1289 & 134.651 & 477 & 147.632 & 679 & 161.231 & 3836 \\
\hline 110.075 & 4963 & 121.560 & 1157 & 135.083 & 4323 & 147.687 & 340 & 161.557 & 658 \\
\hline 110.109 & 11525 & 121.609 & 1005 & 135.120 & 103655 & 147.917 & 458 & 161.631 & 1298 \\
\hline 110.162 & 906 & 121.649 & 438 & 135.538 & 590 & 147.961 & 464 & 161.957 & 400 \\
\hline 110.197 & 1471 & 121.871 & 575 & 135.583 & 714 & 148.002 & 459 & 162.019 & 665 \\
\hline 110.491 & 464 & 121.929 & 920 & 135.930 & 479 & 148.091 & 4044 & 162.105 & 4136 \\
\hline 110.544 & 570 & 121.990 & 545 & 135.991 & 336 & 148.126 & 30041 & 162.141 & 25237 \\
\hline 110.870 & 554 & 122.075 & 4921 & 136.091 & 5384 & 148.231 & 682 & 162.246 & 640 \\
\hline 111.083 & 6468 & 122.110 & 25820 & 136.125 & 21381 & 148.275 & 416 & 162.433 & 454 \\
\hline 111.120 & 28142 & 122.975 & 429 & 136.223 & 521 & 148.336 & 610 & 163.114 & 19515 \\
\hline 111.541 & 611 & 123.083 & 12041 & 136.295 & 447 & 148.756 & 410 & 163.151 & 70214 \\
\hline 111.589 & 355 & 123.120 & 45517 & 137.048 & 496 & 149.099 & 7631 & 163.247 & 2998 \\
\hline 112.089 & 1230 & 124.091 & 29343 & 137.099 & 9474 & 149.135 & 60463 & 163.613 & 617 \\
\hline 112.124 & 2963 & 124.125 & 5288 & 137.135 & 17869 & 149.578 & 622 & 164.064 & 535 \\
\hline 113.092 & 771 & 124.575 & 415 & 137.216 & 448 & 149.619 & 473 & 164.121 & 6766 \\
\hline 113.135 & 3113 & 125.099 & 19461 & 138.106 & 8287 & 149.672 & 502 & 164.155 & 10499 \\
\hline 113.590 & 305 & 125.135 & 9470 & 138.140 & 2773 & 149.966 & 466 & 164.464 & 444 \\
\hline 114.096 & 1868 & 125.198 & 827 & 139.059 & 436 & 150.106 & 4052 & 164.501 & 304 \\
\hline 114.139 & 349 & 126.102 & 2011 & 139.113 & 2368 & 150.140 & 9065 & 165.073 & 1985 \\
\hline 114.596 & 362 & 126.140 & 1418 & 139.150 & 3267 & 151.114 & 9654 & 165.130 & 3958 \\
\hline 115.057 & 18560 & 127.057 & 4236 & 140.116 & 526 & 151.151 & 12628 & 165.166 & 10978 \\
\hline 115.146 & 473 & 127.106 & 464 & 140.155 & 510 & 151.216 & 467 & 166.080 & 1336 \\
\hline 116.065 & 9781 & 127.151 & 2777 & 141.073 & 10125 & 151.251 & 481 & 166.135 & 678 \\
\hline 117.073 & 64317 & 128.065 & 18938 & 141.166 & 720 & 152.066 & 1336 & 166.171 & 1922 \\
\hline 117.475 & 498 & 128.115 & 451 & 142.080 & 11804 & 152.122 & 3950 & 167.088 & 2322 \\
\hline 117.539 & 500 & 129.073 & 40581 & 143.088 & 79937 & 152.157 & 3267 & 167.182 & 695 \\
\hline 117.826 & 407 & 130.080 & 21558 & 143.490 & 475 & 153.072 & 2875 & 168.096 & 1957 \\
\hline 117.873 & 410 & 131.088 & 101242 & 143.536 & 500 & 153.128 & 622 & 169.104 & 7846 \\
\hline 118.080 & 36890 & 131.181 & 3206 & 143.581 & 618 & 153.166 & 1544 & 170.110 & 4225 \\
\hline 118.505 & 483 & 131.469 & 478 & 143.922 & 481 & 154.081 & 2470 & 171.120 & 50573 \\
\hline 118.582 & 472 & 131.503 & 555 & 143.960 & 425 & 155.089 & 8749 & 171.231 & 1785 \\
\hline 118.904 & 488 & 131.553 & 843 & 144.008 & 476 & 156.096 & 6773 & 171.602 & 541 \\
\hline 119.057 & 845 & 131.903 & 645 & 144.095 & 33170 & 157.104 & 50714 & 171.739 & 377 \\
\hline 119.088 & 132461 & 131.972 & 464 & 145.070 & 1471 & 157.200 & 1752 & 172.010 & 443 \\
\hline 119.178 & 3991 & 132.095 & 33647 & 145.104 & 199813 & 157.560 & 462 & 172.126 & 19770 \\
\hline 119.295 & 303 & 132.191 & 1082 & 145.501 & 1044 & 157.981 & 424 & 172.218 & 506 \\
\hline 119.490 & 1076 & 132.255 & 927 & 145.540 & 1240 & 158.111 & 56590 & 173.099 & 2832 \\
\hline 119.539 & 1561 & 132.605 & 416 & 145.585 & 1779 & 158.571 & 265 & 173.135 & 68834 \\
\hline 119.855 & 964 & 133.006 & 476 & 145.634 & 1099 & 159.083 & 5236 & 173.612 & 596 \\
\hline 119.901 & 636 & 133.068 & 2721 & 145.704 & 268 & 159.119 & 130724 & 173.675 & 612 \\
\hline 119.956 & 519 & 133.104 & 119791 & 145.929 & 878 & 159.531 & 673 & 174.106 & 4689 \\
\hline
\end{tabular}


Table S23 (continued). High-resolution mass table for the compound identified as cholesterol.

\begin{tabular}{|c|c|c|c|c|c|c|c|c|c|}
\hline Mass & $\begin{array}{c}\text { Ion } \\
\text { counts }\end{array}$ & Mass & $\begin{array}{c}\text { Ion } \\
\text { counts }\end{array}$ & Mass & $\begin{array}{c}\text { Ion } \\
\text { counts }\end{array}$ & Mass & $\begin{array}{c}\text { Ion } \\
\text { counts }\end{array}$ & Mass & $\begin{array}{c}\text { Ion } \\
\text { counts }\end{array}$ \\
\hline 174.141 & 23175 & 197.135 & 9906 & 223.165 & 430 & 249.254 & 2340 & 276.278 & 38199 \\
\hline 174.201 & 716 & 198.141 & 4447 & 224.160 & 340 & 249.385 & 261 & 276.420 & 3007 \\
\hline 175.114 & 8742 & 199.151 & 57209 & 225.166 & 2918 & 249.942 & 514 & 276.927 & 1102 \\
\hline 175.150 & 24437 & 199.263 & 2228 & 226.171 & 2091 & 250.266 & 367 & 277.001 & 1646 \\
\hline 175.259 & 867 & 199.666 & 517 & 227.181 & 25119 & 251.183 & 374 & 277.283 & 4624 \\
\hline 176.121 & 8424 & 200.156 & 18223 & 228.189 & 30970 & 251.948 & 402 & 277.536 & 948 \\
\hline 176.156 & 6118 & 200.262 & 720 & 228.306 & 1344 & 253.197 & 4432 & 278.042 & 640 \\
\hline 177.130 & 10570 & 201.132 & 1914 & 229.161 & 13234 & 254.203 & 2947 & 278.120 & 487 \\
\hline 177.166 & 13876 & 201.166 & 29046 & 229.196 & 34360 & 255.213 & 97028 & 278.289 & 486 \\
\hline 177.264 & 636 & 201.275 & 1040 & 229.311 & 2059 & 255.343 & 3916 & 278.592 & 530 \\
\hline 178.080 & 940 & 202.171 & 7868 & 229.738 & 613 & 255.791 & 968 & 281.228 & 916 \\
\hline 178.138 & 28630 & 203.145 & 4940 & 229.801 & 388 & 256.216 & 21682 & 282.235 & 499 \\
\hline 178.245 & 1023 & 203.181 & 7110 & 229.861 & 392 & 256.336 & 1727 & 283.244 & 6554 \\
\hline 179.087 & 1051 & 204.152 & 2157 & 230.167 & 5502 & 256.840 & 761 & 284.249 & 1840 \\
\hline 179.143 & 7110 & 204.187 & 2255 & 230.200 & 7231 & 256.910 & 641 & 285.258 & 3427 \\
\hline 179.182 & 7718 & 205.161 & 2528 & 230.300 & 598 & 257.224 & 8370 & 286.265 & 2531 \\
\hline 180.098 & 718 & 205.198 & 16141 & 230.820 & 485 & 257.346 & 518 & 287.275 & 6340 \\
\hline 180.149 & 884 & 206.204 & 11264 & 231.176 & 67741 & 257.410 & 568 & 288.281 & 3046 \\
\hline 180.186 & 1389 & 207.035 & 345 & 231.289 & 2835 & 257.826 & 654 & 289.288 & 852 \\
\hline 181.105 & 1751 & 207.125 & 424 & 231.739 & 763 & 257.925 & 674 & 295.243 & 522 \\
\hline 181.195 & 669 & 207.213 & 9248 & 232.181 & 19668 & 258.232 & 2552 & 297.261 & 2806 \\
\hline 182.111 & 1528 & 208.217 & 2519 & 232.313 & 1130 & 259.208 & 2505 & 298.263 & 1550 \\
\hline 183.119 & 7156 & 209.137 & 779 & 232.740 & 568 & 259.244 & 8655 & 299.275 & 5022 \\
\hline 184.126 & 4319 & 209.224 & 436 & 233.188 & 3859 & 259.350 & 550 & 300.283 & 7575 \\
\hline 185.135 & 45723 & 210.138 & 438 & 233.229 & 8544 & 260.213 & 1651 & 301.290 & 107970 \\
\hline 185.205 & 745 & 211.150 & 6605 & 233.328 & 733 & 260.251 & 12451 & 301.384 & 1311 \\
\hline 185.248 & 1434 & 212.157 & 3842 & 233.766 & 573 & 260.382 & 589 & 301.434 & 4358 \\
\hline 186.142 & 22262 & 213.166 & 164463 & 234.199 & 1673 & 261.259 & 13486 & 301.914 & 915 \\
\hline 186.236 & 730 & 213.282 & 5894 & 234.232 & 2211 & 262.265 & 4788 & 301.978 & 1150 \\
\hline 187.114 & 1764 & 213.650 & 855 & 235.243 & 1181 & 263.269 & 1113 & 302.058 & 839 \\
\hline 187.151 & 45168 & 213.709 & 1899 & 236.250 & 415 & 267.215 & 1284 & 302.148 & 427 \\
\hline 187.263 & 1477 & 214.171 & 43516 & 239.181 & 5283 & 268.216 & 506 & 302.294 & 27082 \\
\hline 188.123 & 1185 & 214.261 & 2378 & 240.188 & 2283 & 269.226 & 3003 & 302.443 & 1369 \\
\hline 188.156 & 11238 & 214.302 & 1821 & 241.197 & 15158 & 270.212 & 1013 & 302.533 & 1414 \\
\hline 189.130 & 4598 & 214.714 & 1354 & 241.317 & 502 & 271.208 & 8976 & 303.052 & 806 \\
\hline 189.166 & 16589 & 215.145 & 4313 & 242.202 & 4184 & 272.213 & 4424 & 303.298 & 3990 \\
\hline 189.250 & 666 & 215.181 & 27361 & 243.178 & 1627 & 273.223 & 48623 & 303.567 & 554 \\
\hline 190.136 & 2330 & 215.288 & 1993 & 243.211 & 3742 & 273.352 & 2138 & 303.709 & 382 \\
\hline 190.171 & 3986 & 215.666 & 869 & 244.184 & 4008 & 273.886 & 587 & 304.304 & 850 \\
\hline 191.089 & 449 & 215.741 & 978 & 245.192 & 5832 & 274.227 & 8974 & 311.275 & 6705 \\
\hline 191.145 & 4312 & 216.151 & 1825 & 245.228 & 10919 & 274.267 & 28323 & 312.281 & 2599 \\
\hline 191.182 & 17866 & 216.186 & 7185 & 246.199 & 9137 & 274.397 & 1717 & 313.290 & 11161 \\
\hline 191.277 & 515 & 216.274 & 571 & 246.233 & 8125 & 274.497 & 666 & 314.297 & 10121 \\
\hline 192.152 & 2571 & 217.161 & 10753 & 246.320 & 571 & 274.892 & 663 & 315.303 & 4740 \\
\hline 192.187 & 4393 & 217.195 & 4774 & 246.386 & 512 & 274.979 & 662 & 316.311 & 2287 \\
\hline 193.198 & 14677 & 218.167 & 5449 & 247.207 & 4200 & 275.117 & 629 & 317.288 & 1122 \\
\hline 193.322 & 445 & 219.176 & 3406 & 247.244 & 52900 & 275.229 & 1954 & 325.291 & 3650 \\
\hline 194.115 & 386 & 219.213 & 14058 & 247.775 & 411 & 275.275 & 170406 & 326.298 & 22418 \\
\hline 194.202 & 3053 & 219.337 & 517 & 247.858 & 627 & 275.406 & 7044 & 326.436 & 711 \\
\hline 195.120 & 1396 & 220.219 & 7622 & 248.248 & 14712 & 275.809 & 795 & 327.304 & 17403 \\
\hline 195.212 & 641 & 221.226 & 2245 & 248.361 & 986 & 275.876 & 966 & 327.437 & 619 \\
\hline 196.127 & 966 & 222.230 & 488 & 248.865 & 561 & 275.940 & 2236 & 328.310 & 6690 \\
\hline
\end{tabular}


Table S23 (continued). High-resolution mass table for the compound identified as cholesterol.

\begin{tabular}{|c|c|c|c|c|c|c|c|c|c|}
\hline Mass & $\begin{array}{c}\text { Ion } \\
\text { counts }\end{array}$ & Mass & $\begin{array}{c}\text { Ion } \\
\text { counts }\end{array}$ & Mass & $\begin{array}{c}\text { Ion } \\
\text { counts }\end{array}$ & Mass & $\begin{array}{c}\text { Ion } \\
\text { counts }\end{array}$ & Mass & $\begin{array}{c}\text { Ion } \\
\text { counts }\end{array}$ \\
\hline 329.320 & 12836 & 352.310 & 927 & 358.322 & 1527 & 371.331 & 85087 & 386.354 & 103884 \\
\hline 329.470 & 554 & 353.321 & 140521 & 359.321 & 482 & 371.483 & 3559 & 386.509 & 4753 \\
\hline 330.323 & 3471 & 353.935 & 672 & 366.330 & 2149 & 371.963 & 502 & 387.163 & 1243 \\
\hline 331.304 & 1396 & 354.061 & 1851 & 367.336 & 3537 & 372.036 & 988 & 387.358 & 31804 \\
\hline 332.307 & 411 & 354.325 & 40349 & 368.190 & 462 & 372.188 & 589 & 387.523 & 1021 \\
\hline 339.306 & 5541 & 354.475 & 1132 & 368.344 & 108854 & 372.334 & 24857 & 387.609 & 454 \\
\hline 340.312 & 5387 & 354.547 & 869 & 368.496 & 4989 & 372.689 & 640 & 387.712 & 837 \\
\hline 341.318 & 2077 & 354.640 & 1342 & 369.073 & 1208 & 373.338 & 4474 & 388.361 & 5538 \\
\hline 342.329 & 4730 & 355.329 & 8013 & 369.181 & 916 & 373.463 & 455 & 389.361 & 322 \\
\hline 343.302 & 5268 & 355.873 & 1339 & 369.347 & 38351 & 374.341 & 376 & 396.400 & 252 \\
\hline 344.304 & 2016 & 355.974 & 685 & 369.510 & 1258 & 382.325 & 756 & & \\
\hline 351.305 & 2475 & 356.331 & 1706 & 369.704 & 811 & 384.339 & 10004 & & \\
\hline 345.312 & 457 & 357.316 & 2642 & 370.350 & 7322 & 385.344 & 4009 & & \\
\hline
\end{tabular}


Table S24. High-resolution mass table for the compound identified as lathosterol.

\begin{tabular}{|c|c|c|c|c|c|c|c|c|c|}
\hline Mass & $\begin{array}{c}\text { Ion } \\
\text { counts }\end{array}$ & Mass & $\begin{array}{c}\text { Ion } \\
\text { counts }\end{array}$ & Mass & $\begin{array}{c}\text { Ion } \\
\text { counts }\end{array}$ & Mass & $\begin{array}{c}\text { Ion } \\
\text { counts }\end{array}$ & Mass & $\begin{array}{c}\text { Ion } \\
\text { counts }\end{array}$ \\
\hline 50.018 & 454 & 87.047 & 538 & 121.069 & 970 & 152.118 & 564 & 189.130 & 981 \\
\hline 51.026 & 933 & 89.039 & 369 & 121.104 & 17713 & 152.158 & 1218 & 189.166 & 2305 \\
\hline 52.033 & 641 & 91.057 & 28014 & 121.203 & 472 & 153.072 & 842 & 190.171 & 886 \\
\hline 53.041 & 4546 & 91.107 & 359 & 122.075 & 872 & 153.152 & 462 & \begin{tabular}{|l|l|}
191.180 \\
\end{tabular} & 1401 \\
\hline 54.049 & 2064 & 91.136 & 565 & 122.111 & 6407 & 154.080 & 738 & 192.186 & 408 \\
\hline 55.021 & 2695 & 92.064 & 6557 & 123.083 & 2268 & 155.088 & 2182 & 193.195 & 994 \\
\hline 55.057 & 38311 & 93.073 & 22173 & 123.119 & 8363 & 156.096 & 1584 & 194.204 & 422 \\
\hline 56.027 & 366 & 93.150 & 528 & 124.091 & 2991 & 157.104 & 7048 & 195.119 & 467 \\
\hline 56.064 & 5645 & 94.079 & 7028 & 124.124 & 1383 & 158.110 & 3221 & 197.134 & 1894 \\
\hline 57.036 & 2999 & 95.052 & 1017 & 125.098 & 1797 & 159.085 & 673 & 198.142 & 938 \\
\hline 57.073 & 24393 & 95.088 & 30188 & 125.135 & 1097 & 159.119 & 12852 & 199.150 & 5623 \\
\hline 57.130 & 554 & 95.144 & 342 & 126.140 & 201 & 159.199 & 418 & 200.156 & 2401 \\
\hline 57.350 & 356 & 95.171 & 718 & 127.058 & 905 & 160.125 & 4636 & 201.166 & 6742 \\
\hline 58.044 & 413 & 96.060 & 1079 & 128.064 & 3337 & 161.099 & 975 & 202.171 & 1808 \\
\hline 58.076 & 1325 & 96.094 & 4618 & 129.072 & 6564 & 161.135 & 15728 & 203.149 & 936 \\
\hline 59.052 & 442 & 97.067 & 2674 & 130.079 & 3205 & 161.255 & 494 & 203.180 & 1803 \\
\hline 60.024 & 1754 & 97.104 & 7864 & 131.088 & 14238 & 162.140 & 3360 & 204.150 & 446 \\
\hline 61.031 & 800 & 98.075 & 2325 & 131.183 & 494 & 163.113 & 1994 & 205.195 & 1306 \\
\hline 63.025 & 440 & 98.109 & 969 & 131.229 & 414 & 163.150 & 2754 & 206.205 & 817 \\
\hline 65.041 & 2645 & 99.081 & 561 & 132.094 & 4306 & 164.120 & 770 & 207.035 & 466 \\
\hline 66.049 & 1267 & 99.119 & 632 & 133.104 & 19299 & 164.155 & 563 & 207.215 & 628 \\
\hline 67.057 & 18954 & 101.062 & 447 & 133.187 & 468 & 165.084 & 1296 & 211.150 & 1536 \\
\hline 67.125 & 458 & 102.053 & 308 & 134.111 & 8868 & 165.129 & 1006 & 212.155 & 618 \\
\hline 68.064 & 5767 & 103.057 & 2345 & 135.083 & 894 & 165.165 & 769 & 213.166 & 25479 \\
\hline 69.036 & 831 & 104.065 & 2287 & 135.119 & 17013 & 166.077 & 277 & 213.287 & 758 \\
\hline 69.073 & 25243 & 105.073 & 29143 & 135.204 & 441 & 166.174 & 430 & 214.169 & 5523 \\
\hline 69.136 & 603 & 106.079 & 8097 & 136.090 & 705 & 167.088 & 766 & 215.180 & 4863 \\
\hline 70.043 & 364 & 107.050 & 735 & 136.125 & 4490 & 168.098 & 555 & 216.185 & 1411 \\
\hline 70.079 & 4070 & 107.088 & 31290 & 137.043 & 557 & 169.103 & 1819 & 217.161 & 2322 \\
\hline 71.052 & 1308 & 108.095 & 6668 & 137.098 & 2210 & 170.110 & 890 & 218.168 & 1572 \\
\hline 71.088 & 10631 & 108.154 & 387 & 137.135 & 2622 & 171.120 & 6650 & 219.175 & 1706 \\
\hline 72.092 & 628 & 109.068 & 1409 & 138.106 & 706 & 172.125 & 2613 & 219.210 & 1080 \\
\hline 73.031 & 1975 & 109.104 & 13846 & 138.142 & 700 & 173.135 & 9072 & 220.201 & 493 \\
\hline 75.028 & 491 & 109.198 & 452 & 139.114 & 666 & 174.141 & 2868 & 225.165 & 491 \\
\hline 77.041 & 8213 & 110.075 & 1371 & 139.149 & 367 & 175.115 & 972 & 226.173 & 409 \\
\hline 78.049 & 2998 & 110.109 & 2076 & 141.072 & 2349 & 175.150 & 3861 & 227.181 & 4262 \\
\hline 79.057 & 22060 & 111.082 & 2280 & 142.081 & 2244 & 176.120 & 651 & 228.189 & 3826 \\
\hline 79.129 & 505 & 111.119 & 3181 & 143.088 & 7605 & 176.156 & 833 & 229.161 & 3982 \\
\hline 80.064 & 3812 & 112.090 & 941 & 144.096 & 3045 & 177.129 & 1170 & 229.196 & 13411 \\
\hline 81.036 & 421 & 112.126 & 675 & 145.104 & 17696 & 177.165 & 1394 & 229.312 & 531 \\
\hline 81.073 & 28615 & 113.089 & 385 & 145.198 & 465 & 178.137 & 1797 & 230.167 & 1386 \\
\hline 81.139 & 687 & 113.138 & 421 & 146.110 & 5220 & 179.087 & 268 & 230.200 & 2687 \\
\hline 82.044 & 411 & 114.077 & 449 & 147.082 & 565 & 179.140 & 518 & 231.176 & 15887 \\
\hline 82.079 & 5598 & 115.057 & 3731 & 147.119 & 20984 & 179.179 & 360 & 231.287 & 588 \\
\hline 83.052 & 1940 & 116.064 & 1847 & 147.215 & 552 & 181.104 & 544 & 232.180 & 4259 \\
\hline 83.088 & 13725 & 117.073 & 10554 & 148.125 & 5205 & 182.113 & 489 & 233.189 & 1304 \\
\hline 83.156 & 556 & 118.079 & 4576 & 149.099 & 1377 & 183.119 & 1533 & 233.224 & 872 \\
\hline 84.059 & 1882 & 119.088 & 23797 & 149.135 & 7222 & 184.125 & 781 & 235.213 & 361 \\
\hline 84.094 & 2054 & 119.143 & 283 & 150.138 & 1549 & 185.135 & 5707 & 236.218 & 595 \\
\hline 85.032 & 341 & 119.177 & 650 & 151.114 & 2664 & 186.140 & 2401 & 239.180 & 1068 \\
\hline 85.068 & 1346 & 120.095 & 9406 & 151.151 & 1196 & 187.150 & 5564 & 240.188 & 504 \\
\hline 85.104 & 3198 & 120.187 & 429 & 152.065 & 605 & 188.155 & 1422 & 241.196 & 1984 \\
\hline
\end{tabular}


Table S24 (continued). High-resolution mass table for the compound identified as lathosterol.

\begin{tabular}{|c|c|c|c|c|c|c|c|c|c|}
\hline Mass & $\begin{array}{c}\text { Ion } \\
\text { counts }\end{array}$ & Mass & $\begin{array}{c}\text { Ion } \\
\text { counts }\end{array}$ & Mass & $\begin{array}{c}\text { Ion } \\
\text { counts }\end{array}$ & Mass & $\begin{array}{c}\text { Ion } \\
\text { counts }\end{array}$ & Mass & $\begin{array}{c}\text { Ion } \\
\text { counts }\end{array}$ \\
\hline 242.202 & 916 & 256.392 & 535 & 283.240 & 693 & 328.306 & 376 & 372.333 & 4709 \\
\hline 243.209 & 1153 & 257.220 & 2252 & 285.238 & 535 & 329.287 & 991 & 373.337 & 882 \\
\hline 244.182 & 1385 & 258.202 & 437 & 286.264 & 444 & 339.301 & 425 & 381.353 & 474 \\
\hline 245.190 & 1847 & 259.242 & 3850 & 287.271 & 942 & 351.305 & 763 & 382.348 & 610 \\
\hline 245.226 & 2929 & 260.249 & 2689 & 288.262 & 424 & 353.320 & 7908 & 384.337 & 3411 \\
\hline 246.199 & 4998 & 261.256 & 1673 & 299.269 & 454 & 354.322 & 2615 & 385.342 & 2273 \\
\hline 247.205 & 2275 & 262.263 & 699 & 301.289 & 1391 & 355.328 & 634 & 386.352 & 27558 \\
\hline 247.242 & 3951 & 267.217 & 389 & 302.282 & 321 & 357.318 & 365 & 386.503 & 1285 \\
\hline 248.244 & 1187 & 269.212 & 1252 & 311.274 & 399 & 366.329 & 404 & 387.356 & 8107 \\
\hline 253.196 & 1456 & 270.210 & 431 & 313.290 & 1185 & 367.331 & 1262 & 388.362 & 1522 \\
\hline 254.202 & 654 & 271.206 & 4582 & 314.292 & 622 & 368.341 & 4377 & 398.353 & 328 \\
\hline 255.211 & 41099 & 272.212 & 1594 & 315.308 & 448 & 369.325 & 3580 & 399.360 & 1250 \\
\hline 255.790 & 397 & 273.222 & 7838 & 325.289 & 463 & 370.332 & 750 & 400.368 & 2743 \\
\hline 256.215 & 8860 & 274.227 & 2113 & 326.297 & 485 & 371.330 & 16871 & 401.369 & 837 \\
\hline 256.302 & 480 & 275.273 & 1781 & 327.300 & 693 & 371.476 & 548 & &
\end{tabular}


Table S25. High-resolution mass table for the compound identified as hexadecanoic acid tetradecyl ester.

\begin{tabular}{|c|c|c|c|c|c|c|c|}
\hline Mass & $\begin{array}{c}\text { Ion } \\
\text { counts }\end{array}$ & Mass & $\begin{array}{c}\text { Ion } \\
\text { counts }\end{array}$ & Mass & $\begin{array}{c}\text { Ion } \\
\text { counts }\end{array}$ & Mass & $\begin{array}{c}\text { Ion } \\
\text { counts }\end{array}$ \\
\hline 50.018 & 956 & 58.818 & 681 & 68.674 & 461 & 73.698 & 428 \\
\hline 51.026 & 1513 & 58.841 & 595 & 68.708 & 458 & 73.912 & 509 \\
\hline 52.033 & 850 & 58.875 & 937 & 68.920 & 457 & 73.938 & 264 \\
\hline 53.042 & 11135 & 58.909 & 437 & 68.940 & 356 & 73.959 & 465 \\
\hline 54.049 & 36516 & 59.052 & 6597 & 68.967 & 464 & 74.038 & 11657 \\
\hline 54.112 & 849 & 59.081 & 1008 & 69.037 & 3677 & 74.101 & 449 \\
\hline 54.316 & 384 & 59.106 & 869 & 69.073 & 308925 & 74.183 & 472 \\
\hline 54.800 & 363 & 59.344 & 425 & 69.344 & 1395 & 75.044 & 2519 \\
\hline 55.021 & 17530 & 59.365 & 497 & 69.373 & 1479 & 77.042 & 2939 \\
\hline 55.057 & 333835 & 59.566 & 386 & 69.401 & 2694 & 78.049 & 1951 \\
\hline 55.082 & 688 & 59.622 & 329 & 69.483 & 706 & 79.057 & 11049 \\
\hline 55.097 & 2771 & 59.836 & 345 & 69.672 & 2427 & 80.065 & 5554 \\
\hline 55.299 & 1934 & 59.865 & 377 & 69.946 & 1707 & 81.037 & 821 \\
\hline 55.325 & 1883 & 60.024 & 62551 & 70.044 & 4645 & 81.073 & 55412 \\
\hline 55.352 & 3084 & 60.059 & 870 & 70.080 & 159486 & 81.404 & 397 \\
\hline 55.424 & 514 & 60.088 & 1882 & 70.383 & 774 & 81.432 & 325 \\
\hline 55.567 & 2323 & 60.123 & 850 & 70.415 & 1204 & 81.458 & 332 \\
\hline 55.600 & 2465 & 60.323 & 615 & 70.443 & 1573 & 81.714 & 401 \\
\hline 55.824 & 2080 & 60.351 & 420 & 70.494 & 810 & 82.044 & 1093 \\
\hline 55.854 & 1716 & 60.377 & 472 & 70.689 & 1080 & 82.081 & 122060 \\
\hline 56.028 & 3530 & 60.558 & 482 & 70.725 & 1230 & 82.375 & 425 \\
\hline 56.065 & 161374 & 60.597 & 506 & 70.947 & 621 & 82.408 & 689 \\
\hline 56.092 & 578 & 60.812 & 425 & 70.986 & 976 & 82.439 & 752 \\
\hline 56.304 & 1936 & 60.855 & 488 & 71.052 & 10755 & 82.467 & 702 \\
\hline 56.336 & 1742 & 61.031 & 86394 & 71.088 & 333598 & 82.719 & 517 \\
\hline 56.362 & 2126 & 61.293 & 556 & 71.365 & 1640 & 82.770 & 437 \\
\hline 56.554 & 1314 & 61.314 & 542 & 71.393 & 1790 & 83.052 & 17745 \\
\hline 56.590 & 1665 & 61.346 & 1045 & 71.426 & 2815 & 83.088 & 305419 \\
\hline 56.625 & 1268 & 61.374 & 693 & 71.507 & 805 & 83.388 & 1892 \\
\hline 56.831 & 1651 & 61.578 & 587 & 71.670 & 1895 & 83.418 & 1648 \\
\hline 57.036 & 10895 & 61.611 & 573 & 71.700 & 2171 & 83.453 & 2795 \\
\hline 57.073 & 554016 & 61.826 & 446 & 71.960 & 1455 & 83.541 & 456 \\
\hline 57.112 & 12583 & 61.858 & 436 & 72.023 & 970 & 83.754 & 2286 \\
\hline 57.146 & 12721 & 61.889 & 399 & 72.059 & 1600 & 84.060 & 34491 \\
\hline 57.191 & 3866 & 62.035 & 2494 & 72.092 & 17378 & 84.096 & 117831 \\
\hline 57.322 & 3926 & 62.083 & 461 & 72.166 & 458 & 84.171 & 3530 \\
\hline 57.346 & 3651 & 62.140 & 429 & 72.209 & 612 & 84.340 & 768 \\
\hline 57.372 & 5758 & 63.028 & 759 & 72.246 & 852 & 84.393 & 1315 \\
\hline 57.426 & 1687 & 65.042 & 2800 & 72.275 & 796 & 84.427 & 1210 \\
\hline 57.444 & 1162 & 66.049 & 4566 & 72.495 & 573 & 84.667 & 536 \\
\hline 57.583 & 3879 & 67.057 & 64074 & 72.527 & 386 & 84.707 & 629 \\
\hline 57.610 & 4467 & 67.126 & 1708 & 72.556 & 452 & 84.749 & 599 \\
\hline 57.637 & 4104 & 67.164 & 704 & 72.778 & 425 & 84.980 & 394 \\
\hline 57.839 & 3327 & 67.360 & 512 & 72.815 & 374 & 85.032 & 1322 \\
\hline 57.870 & 3293 & 67.662 & 430 & 73.031 & 108534 & 85.067 & 8322 \\
\hline 58.044 & 6588 & 67.911 & 409 & 73.068 & 6316 & 85.104 & 204368 \\
\hline 58.076 & 26901 & 68.029 & 593 & 73.310 & 871 & 85.152 & 1758 \\
\hline 58.112 & 2419 & 68.065 & 77947 & 73.340 & 669 & 85.407 & 1175 \\
\hline 58.135 & 2868 & 68.364 & 272 & 73.366 & 1127 & 85.437 & 1187 \\
\hline 58.377 & 1965 & 68.405 & 518 & 73.390 & 1089 & 85.476 & 1701 \\
\hline 58.571 & 1379 & 68.450 & 364 & 73.634 & 694 & 85.507 & 1425 \\
\hline 58.611 & 1526 & 68.641 & 450 & 73.662 & 541 & 85.562 & 452 \\
\hline
\end{tabular}


Table S25 (continued). High-resolution mass table for the compound identified as hexadecanoic acid tetradecyl ester.

\begin{tabular}{|c|c|c|c|c|c|c|c|}
\hline Mass & $\begin{array}{c}\text { Ion } \\
\text { counts }\end{array}$ & Mass & $\begin{array}{c}\text { Ion } \\
\text { counts }\end{array}$ & Mass & $\begin{array}{c}\text { Ion } \\
\text { counts }\end{array}$ & Mass & $\begin{array}{c}\text { Ion } \\
\text { counts }\end{array}$ \\
\hline 85.756 & 1429 & 99.445 & 1071 & 115.078 & 28256 & 138.107 & 1621 \\
\hline 86.040 & 2009 & 99.485 & 802 & 115.118 & 524 & 138.143 & 10230 \\
\hline 86.073 & 2031 & 99.727 & 419 & 115.164 & 630 & 139.115 & 9000 \\
\hline 86.108 & 12953 & 99.771 & 641 & 116.086 & 23198 & 139.151 & 21864 \\
\hline 86.150 & 385 & 99.825 & 552 & 117.092 & 6259 & 139.207 & 517 \\
\hline 86.375 & 458 & 100.055 & 2690 & 118.082 & 686 & 139.238 & 779 \\
\hline 86.425 & 482 & 100.090 & 1477 & 119.089 & 2461 & 140.120 & 1728 \\
\hline 86.668 & 322 & 100.123 & 3475 & 120.095 & 1192 & 140.158 & 21401 \\
\hline 86.699 & 405 & 100.444 & 433 & 121.104 & 7721 & 140.224 & 403 \\
\hline 87.047 & 29797 & 101.063 & 24772 & 122.111 & 2322 & 140.263 & 562 \\
\hline 87.083 & 2007 & 101.100 & 1172 & 123.083 & 2736 & 141.082 & 1186 \\
\hline 87.111 & 883 & 101.136 & 754 & 123.120 & 11169 & 141.130 & 888 \\
\hline 88.054 & 4108 & 101.781 & 341 & 124.090 & 2332 & 141.166 & 8669 \\
\hline 89.063 & 6623 & 102.070 & 6720 & 124.127 & 15813 & 142.088 & 572 \\
\hline 91.058 & 4380 & 102.119 & 379 & 124.225 & 473 & 142.168 & 849 \\
\hline 92.065 & 1419 & 103.078 & 7552 & 125.099 & 11750 & 143.110 & 13622 \\
\hline 93.073 & 7470 & 104.071 & 890 & 125.135 & 62749 & 143.152 & 213 \\
\hline 94.080 & 4114 & 105.073 & 2735 & 125.542 & 532 & 144.115 & 2805 \\
\hline 95.053 & 1296 & 106.080 & 933 & 125.578 & 385 & 145.106 & 1542 \\
\hline 95.089 & 46897 & 107.052 & 311 & 125.619 & 485 & 145.121 & 1829 \\
\hline 95.449 & 399 & 107.089 & 6310 & 126.063 & 424 & 146.111 & 583 \\
\hline 96.060 & 5288 & 108.095 & 2608 & 126.105 & 2710 & 147.120 & 1950 \\
\hline 96.096 & 62147 & 109.067 & 1557 & 126.143 & 33742 & 148.127 & 922 \\
\hline 96.424 & 469 & 109.104 & 23749 & 126.591 & 308 & 149.135 & 5315 \\
\hline 96.450 & 448 & 109.179 & 605 & 126.639 & 452 & 150.140 & 1110 \\
\hline 96.499 & 604 & 110.076 & 3522 & 127.078 & 2419 & 151.114 & 1704 \\
\hline 96.754 & 406 & 110.112 & 28200 & 127.114 & 1330 & 151.152 & 2410 \\
\hline 96.798 & 532 & 111.084 & 19128 & 127.150 & 13333 & 152.122 & 1364 \\
\hline 96.842 & 445 & 111.120 & 133499 & 127.243 & 504 & 152.159 & 6705 \\
\hline 97.068 & 26624 & 111.463 & 897 & 127.304 & 449 & 153.130 & 6033 \\
\hline 97.104 & 256162 & 111.502 & 894 & 128.076 & 1463 & 153.166 & 11127 \\
\hline 97.156 & 2505 & 111.539 & 1690 & 128.120 & 316 & 154.137 & 1465 \\
\hline 97.426 & 2004 & 111.860 & 1049 & 128.153 & 1179 & 154.174 & 12065 \\
\hline 97.461 & 1646 & 112.091 & 9725 & 129.094 & 64611 & 155.100 & 717 \\
\hline 97.489 & 2309 & 112.127 & 53437 & 129.466 & 450 & 155.147 & 664 \\
\hline 97.520 & 2383 & 112.176 & 1170 & 129.505 & 365 & 155.181 & 5013 \\
\hline 97.592 & 389 & 112.473 & 478 & 129.541 & 529 & 156.116 & 474 \\
\hline 97.809 & 2296 & 112.548 & 1390 & 129.585 & 470 & 156.183 & 465 \\
\hline 98.076 & 43211 & 112.607 & 798 & 129.934 & 461 & 157.125 & 16070 \\
\hline 98.111 & 78541 & 112.827 & 398 & 130.099 & 8877 & 157.219 & 467 \\
\hline 98.163 & 3527 & 112.903 & 785 & 130.190 & 450 & 158.130 & 3370 \\
\hline 98.386 & 650 & 113.063 & 2083 & 131.107 & 3794 & 159.137 & 2353 \\
\hline 98.429 & 1770 & 113.098 & 2426 & 132.094 & 571 & 160.126 & 501 \\
\hline 98.481 & 2211 & 113.135 & 21608 & 133.104 & 2122 & 161.136 & 1356 \\
\hline 98.759 & 1341 & 113.219 & 1377 & 134.112 & 1259 & 162.138 & 376 \\
\hline 98.802 & 1465 & 113.277 & 671 & 135.120 & 7563 & 163.151 & 3367 \\
\hline 99.047 & 1982 & 113.543 & 488 & 136.087 & 435 & 164.158 & 1405 \\
\hline 99.081 & 6517 & 113.585 & 582 & 136.126 & 1851 & 165.131 & 1447 \\
\hline 99.119 & 39280 & 113.935 & 482 & 137.055 & 409 & 165.166 & 1740 \\
\hline 99.194 & 1634 & 114.070 & 3025 & 137.099 & 2072 & 166.136 & 455 \\
\hline 99.388 & 398 & 114.139 & 2034 & 137.136 & 5527 & 166.174 & 6002 \\
\hline
\end{tabular}


Table S25 (continued). High-resolution mass table for the compound identified as hexadecanoic acid tetradecyl ester.

\begin{tabular}{|c|c|c|c|c|c|c|c|}
\hline Mass & $\begin{array}{c}\text { Ion } \\
\text { counts }\end{array}$ & Mass & $\begin{array}{c}\text { Ion } \\
\text { counts }\end{array}$ & Mass & $\begin{array}{c}\text { Ion } \\
\text { counts }\end{array}$ & Mass & $\begin{array}{c}\text { Ion } \\
\text { counts }\end{array}$ \\
\hline 167.146 & 3776 & 197.226 & 11238 & 230.222 & 28126 & 258.470 & 3322 \\
\hline 167.182 & 7279 & 198.146 & 568 & 230.296 & 1627 & 258.853 & 2043 \\
\hline 168.153 & 1089 & 198.231 & 1421 & 230.345 & 2576 & 258.933 & 2769 \\
\hline 168.190 & 34798 & 199.172 & 11119 & 230.763 & 1519 & 259.255 & 5930 \\
\hline 168.302 & 992 & 200.178 & 4195 & 230.825 & 1299 & 259.466 & 2128 \\
\hline 169.127 & 673 & 201.187 & 19082 & 231.225 & 3736 & 259.972 & 1547 \\
\hline 169.194 & 6401 & 201.313 & 545 & 231.309 & 1017 & 260.257 & 394 \\
\hline 170.199 & 432 & 202.191 & 2707 & 231.759 & 657 & 260.418 & 781 \\
\hline 171.141 & 17689 & 203.186 & 533 & 231.818 & 613 & 260.496 & 1071 \\
\hline 171.236 & 543 & 205.199 & 818 & 232.229 & 648 & 260.999 & 630 \\
\hline 172.145 & 2906 & 207.033 & 365 & 232.321 & 518 & 261.093 & 534 \\
\hline 173.153 & 1933 & 207.173 & 816 & 232.763 & 481 & 261.432 & 440 \\
\hline 175.150 & 684 & 208.184 & 2129 & 233.229 & 510 & 261.502 & 484 \\
\hline 176.159 & 326 & 209.193 & 3205 & 235.226 & 503 & 261.569 & 513 \\
\hline 177.166 & 1716 & 210.199 & 908 & 236.215 & 3042 & 267.268 & 1195 \\
\hline 178.170 & 540 & 210.236 & 2528 & 237.223 & 4410 & 269.249 & 6916 \\
\hline 179.146 & 810 & 211.208 & 27383 & 238.228 & 1541 & 270.253 & 1866 \\
\hline 179.180 & 856 & 211.313 & 817 & 239.239 & 50121 & 271.264 & 2273 \\
\hline 180.189 & 2117 & 212.212 & 4717 & 239.367 & 2149 & 272.267 & 381 \\
\hline 181.162 & 2739 & 213.187 & 25379 & 239.825 & 533 & 279.271 & 417 \\
\hline 181.197 & 2351 & 213.305 & 747 & 240.242 & 8956 & 283.264 & 1663 \\
\hline 182.168 & 671 & 214.191 & 4128 & 240.365 & 626 & 284.272 & 2004 \\
\hline 182.206 & 4195 & 215.202 & 3820 & 240.937 & 351 & 285.280 & 4053 \\
\hline 183.177 & 3692 & 216.204 & 619 & 241.218 & 11492 & 286.282 & 813 \\
\hline 183.211 & 1219 & 217.202 & 499 & 242.225 & 6182 & 294.295 & 289 \\
\hline 184.183 & 690 & 218.201 & 345 & 242.354 & 563 & 297.280 & 2860 \\
\hline 185.156 & 27062 & 219.213 & 3036 & 243.234 & 28374 & 298.287 & 1044 \\
\hline 185.271 & 808 & 220.218 & 1161 & 243.353 & 1265 & 307.301 & 594 \\
\hline 185.640 & 352 & 221.229 & 1277 & 244.237 & 4777 & 311.297 & 809 \\
\hline 186.160 & 4018 & 222.204 & 502 & 245.241 & 837 & 312.305 & 1251 \\
\hline 187.170 & 2207 & 222.235 & 766 & 247.243 & 423 & 325.310 & 1410 \\
\hline 188.165 & 384 & 223.209 & 1202 & 251.239 & 624 & 326.315 & 517 \\
\hline 189.167 & 401 & 224.252 & 10699 & 252.281 & 662 & 353.336 & 1201 \\
\hline 190.172 & 350 & 225.224 & 4909 & 253.249 & 550 & 367.356 & 364 \\
\hline 191.182 & 2657 & 225.256 & 3206 & 254.256 & 554 & 368.345 & 747 \\
\hline 192.189 & 1813 & 226.228 & 1667 & 255.233 & 4059 & 369.335 & 490 \\
\hline 193.161 & 528 & 227.203 & 8775 & 256.241 & 49183 & 371.327 & 338 \\
\hline 193.197 & 1012 & 228.210 & 26403 & 256.823 & 680 & 384.345 & 322 \\
\hline 194.205 & 5343 & 228.329 & 1020 & 257.249 & 312224 & 409.406 & 1030 \\
\hline 195.178 & 1573 & 228.823 & 427 & 257.778 & 1957 & 410.402 & 353 \\
\hline 195.211 & 2391 & 229.218 & 178977 & 257.852 & 3981 & 452.457 & 9839 \\
\hline 196.221 & 46854 & 229.718 & 1199 & 258.040 & 607 & 452.604 & 602 \\
\hline 196.779 & 504 & 229.766 & 1246 & 258.252 & 54115 & 453.460 & 3381 \\
\hline 197.192 & 1511 & 229.826 & 2439 & 258.385 & 5096 & 454.462 & 495 \\
\hline
\end{tabular}


Table S26. High-resolution mass table for the compound identified as $\mathrm{C}_{31} \mathrm{H}_{62} \mathrm{O}_{2}$.

\begin{tabular}{|c|c|c|c|c|c|c|c|c|c|}
\hline Mass & $\begin{array}{c}\text { Ion } \\
\text { counts }\end{array}$ & Mass & $\begin{array}{c}\text { Ion } \\
\text { counts }\end{array}$ & Mass & $\begin{array}{c}\text { Ion } \\
\text { counts }\end{array}$ & Mass & $\begin{array}{c}\text { Ion } \\
\text { counts }\end{array}$ & Mass & $\begin{array}{c}\text { Ion } \\
\text { counts }\end{array}$ \\
\hline 50.018 & 462 & 68.065 & 18436 & 85.480 & 464 & 112.091 & 4554 & 144.114 & 633 \\
\hline 51.026 & 786 & 68.136 & 385 & 86.039 & 401 & 112.126 & 10719 & 145.105 & 822 \\
\hline 52.033 & 416 & 69.037 & 1055 & 86.074 & 480 & 112.194 & 601 & 147.120 & 798 \\
\hline 53.042 & 3566 & 69.073 & 84325 & 86.107 & 3954 & 113.063 & 480 & 148.125 & 333 \\
\hline 54.049 & 8760 & 69.374 & 275 & 87.047 & 4703 & 113.097 & 1017 & 149.135 & 2499 \\
\hline 55.021 & 5416 & 69.406 & 582 & 87.082 & 544 & 113.135 & 8539 & 150.142 & 552 \\
\hline 55.057 & 92607 & 69.443 & 481 & 88.053 & 664 & 114.070 & 2186 & 151.150 & 1967 \\
\hline 55.115 & 2575 & 69.665 & 493 & 89.062 & 706 & 114.138 & 714 & 152.158 & 3105 \\
\hline 55.325 & 751 & 69.710 & 416 & 91.058 & 2402 & 115.078 & 5691 & 153.130 & 2166 \\
\hline 55.362 & 566 & 69.943 & 423 & 92.065 & 796 & 116.086 & 4869 & 153.166 & 3511 \\
\hline 55.387 & 526 & 70.044 & 1474 & 93.073 & 3122 & 117.092 & 2183 & 154.174 & 3944 \\
\hline 55.532 & 321 & 70.080 & 43698 & 94.080 & 1841 & 118.087 & 346 & 155.101 & 377 \\
\hline 55.568 & 463 & 70.408 & 346 & 95.052 & 493 & 119.088 & 1173 & 155.181 & 3092 \\
\hline 55.602 & 462 & 70.444 & 352 & 95.088 & 18692 & 120.093 & 420 & 156.178 & 328 \\
\hline 55.800 & 413 & 70.468 & 389 & 95.142 & 269 & 121.104 & 3063 & 157.125 & 4865 \\
\hline 55.831 & 524 & 71.052 & 3317 & 95.165 & 470 & 122.111 & 895 & 158.130 & 1134 \\
\hline 56.029 & 1688 & 71.088 & 92614 & 96.060 & 1173 & 123.083 & 726 & 159.123 & 820 \\
\hline 56.065 & 52073 & 71.369 & 421 & 96.096 & 14713 & 123.119 & 5559 & 161.134 & 480 \\
\hline 56.308 & 409 & 71.393 & 597 & 97.068 & 7213 & 124.091 & 849 & 163.150 & 1152 \\
\hline 56.334 & 411 & 71.444 & 588 & 97.104 & 63208 & 124.127 & 3992 & 164.158 & 364 \\
\hline 56.355 & 484 & 71.665 & 458 & 97.462 & 484 & 125.099 & 3838 & 165.167 & 1152 \\
\hline 56.384 & 412 & 71.715 & 396 & 97.502 & 527 & 125.135 & 14354 & 166.173 & 1682 \\
\hline 56.827 & 410 & 71.948 & 396 & 97.791 & 477 & 125.217 & 489 & 167.145 & 2144 \\
\hline 56.871 & 382 & 72.022 & 333 & 98.075 & 8276 & 126.105 & 936 & 167.182 & 2528 \\
\hline 57.036 & 3724 & 72.059 & 556 & 98.111 & 15602 & 126.142 & 7435 & 168.150 & 602 \\
\hline 57.073 & 169009 & 72.092 & 4963 & 98.181 & 588 & 127.078 & 806 & 168.190 & 5989 \\
\hline 57.322 & 864 & 73.031 & 41531 & 98.225 & 393 & 127.117 & 348 & 169.195 & 2197 \\
\hline 57.346 & 1130 & 73.068 & 2279 & 98.462 & 376 & 127.150 & 6134 & 171.140 & 2035 \\
\hline 57.372 & 1563 & 73.413 & 371 & 99.048 & 490 & 128.066 & 765 & 172.143 & 338 \\
\hline 57.598 & 1365 & 74.038 & 3885 & 99.082 & 1778 & 128.154 & 556 & 173.143 & 607 \\
\hline 57.640 & 824 & 75.041 & 1155 & 99.119 & 14130 & 129.094 & 11968 & 177.166 & 2001 \\
\hline 57.674 & 387 & 77.042 & 1822 & 99.180 & 418 & 130.100 & 3061 & 178.173 & 454 \\
\hline 57.862 & 986 & 78.049 & 1278 & 99.206 & 359 & 131.107 & 1553 & \begin{tabular}{|l|}
179.181 \\
\end{tabular} & 1069 \\
\hline 57.893 & 613 & 79.057 & 3966 & 99.484 & 297 & 133.103 & 802 & 180.189 & 5607 \\
\hline 58.044 & 1706 & 80.065 & 1892 & 100.056 & 898 & 134.111 & 357 & 181.163 & 927 \\
\hline 58.076 & 7815 & 81.038 & 289 & 100.122 & 1248 & 135.074 & 386 & 181.196 & 2294 \\
\hline 58.125 & 687 & 81.073 & 19886 & 101.063 & 20109 & 135.119 & 2675 & 182.204 & 3063 \\
\hline 58.169 & 426 & 81.147 & 572 & 101.110 & 446 & 136.125 & 675 & 183.212 & 1979 \\
\hline 58.325 & 425 & 82.042 & 316 & 101.148 & 480 & 137.046 & 426 & 185.156 & 10582 \\
\hline 58.356 & 493 & 82.080 & 26741 & 102.069 & 2829 & 137.098 & 766 & 186.160 & 2033 \\
\hline 58.391 & 445 & 82.147 & 653 & 103.078 & 1720 & 137.135 & 3108 & 187.170 & 1320 \\
\hline 58.577 & 411 & 83.052 & 8008 & 105.073 & 1751 & 138.105 & 552 & 191.182 & 808 \\
\hline 58.627 & 412 & 83.088 & 74023 & 106.079 & 645 & 138.142 & 2439 & 192.190 & 307 \\
\hline 59.052 & 2586 & 83.386 & 486 & 107.089 & 2211 & 139.114 & 4746 & 193.200 & 515 \\
\hline 60.024 & 8178 & 83.418 & 356 & 108.096 & 1167 & 139.151 & 5798 & 194.204 & 788 \\
\hline 61.031 & 15786 & 83.468 & 492 & 109.067 & 609 & 140.120 & 710 & 195.175 & 2239 \\
\hline 61.089 & 371 & 84.059 & 5167 & 109.104 & 11067 & 140.158 & 5071 & 195.210 & 867 \\
\hline 62.033 & 411 & 84.096 & 26045 & 110.075 & 1284 & 141.076 & 561 & 196.220 & 6962 \\
\hline 65.041 & 1145 & 84.169 & 573 & 110.111 & 6988 & 141.166 & 4160 & 197.192 & 1547 \\
\hline 66.050 & 1397 & 85.067 & 2662 & 111.083 & 7110 & 142.086 & 404 & 197.226 & 2990 \\
\hline 67.057 & 18216 & 85.104 & 57507 & 111.120 & 32928 & 142.172 & 477 & 198.186 & 427 \\
\hline 67.130 & 433 & 85.439 & 244 & 111.503 & 459 & 143.108 & 3055 & 198.233 & 389 \\
\hline
\end{tabular}


Table S26 (continued). High-resolution mass table for the compound identified as $\mathrm{C}_{31} \mathrm{H}_{62} \mathrm{O}_{2}$.

\begin{tabular}{|c|c|c|c|c|c|c|c|c|c|}
\hline Mass & $\begin{array}{c}\text { Ion } \\
\text { counts }\end{array}$ & Mass & $\begin{array}{c}\text { Ion } \\
\text { counts }\end{array}$ & Mass & $\begin{array}{c}\text { Ion } \\
\text { counts }\end{array}$ & Mass & $\begin{array}{c}\text { Ion } \\
\text { counts }\end{array}$ & Mass & $\begin{array}{c}\text { Ion } \\
\text { counts }\end{array}$ \\
\hline 199.170 & 2525 & 214.191 & 1776 & 230.221 & 3196 & 244.339 & 558 & 272.267 & 4427 \\
\hline 200.171 & 417 & 215.202 & 11699 & 231.222 & 520 & 245.240 & 983 & 273.270 & 544 \\
\hline 201.188 & 1004 & 216.206 & 1902 & 233.230 & 498 & 247.243 & 382 & 283.263 & 1000 \\
\hline 205.196 & 1413 & 217.210 & 338 & 234.236 & 373 & 251.240 & 806 & 284.269 & 459 \\
\hline 206.202 & 913 & 219.215 & 338 & 235.243 & 958 & 252.282 & 484 & 285.282 & 600 \\
\hline 207.034 & 475 & 222.233 & 396 & 236.227 & 311 & 253.253 & 3068 & 297.280 & 1911 \\
\hline 207.212 & 2008 & 223.208 & 1718 & 237.222 & 721 & 254.259 & 480 & 298.287 & 530 \\
\hline 208.219 & 1759 & 224.251 & 4140 & 238.268 & 471 & 255.230 & 899 & 311.296 & 429 \\
\hline 209.195 & 1043 & 225.223 & 3772 & 239.238 & 1168 & 256.242 & 989 & 325.309 & 1062 \\
\hline 209.229 & 1675 & 225.254 & 2475 & 241.218 & 2839 & 257.249 & 7597 & 353.336 & 695 \\
\hline 210.235 & 2199 & 226.232 & 820 & 242.225 & 3388 & 258.251 & 1300 & 409.404 & 1392 \\
\hline 211.206 & 1925 & 227.203 & 1012 & 243.233 & 48754 & 269.248 & 4017 & 410.406 & 392 \\
\hline 211.243 & 1432 & 228.210 & 2298 & 243.356 & 1855 & 270.256 & 1942 & 466.473 & 1552 \\
\hline 212.221 & 479 & 229.217 & 17792 & 243.840 & 559 & 271.264 & 23764 & 467.476 & 476 \\
\hline 213.186 & 7756 & 229.335 & 559 & 244.236 & 8281 & 271.389 & 737 & &
\end{tabular}


Table S27. High-resolution mass table for the compound identified as $\mathrm{C}_{28} \mathrm{H}_{48} \mathrm{O}$.

\begin{tabular}{|c|c|c|c|c|c|c|c|c|c|}
\hline Mass & $\begin{array}{c}\text { Ion } \\
\text { counts }\end{array}$ & Mass & $\begin{array}{c}\text { Ion } \\
\text { counts }\end{array}$ & Mass & $\begin{array}{c}\text { Ion } \\
\text { counts }\end{array}$ & Mass & $\begin{array}{c}\text { Ion } \\
\text { counts }\end{array}$ & Mass & $\begin{array}{c}\text { Ion } \\
\text { counts }\end{array}$ \\
\hline 50.018 & 470 & 93.073 & 12951 & 135.119 & 9594 & \begin{tabular}{|l|}
181.103 \\
\end{tabular} & 255 & 251.943 & 338 \\
\hline 51.026 & 710 & 94.079 & 3029 & 136.126 & 3010 & \begin{tabular}{|l|}
183.120 \\
\end{tabular} & 975 & 253.196 & 712 \\
\hline 52.033 & 362 & 95.052 & 493 & 137.047 & 337 & 184.124 & 409 & 255.211 & 1815 \\
\hline 53.041 & 2712 & 95.088 & 18345 & 137.099 & 770 & 185.134 & 2880 & 256.221 & 591 \\
\hline 54.049 & 1362 & 95.174 & 473 & 137.134 & 1410 & 186.140 & 1114 & 257.232 & 568 \\
\hline 55.020 & 1687 & 96.061 & 604 & 138.106 & 1083 & 187.150 & 3697 & 258.200 & 476 \\
\hline 55.057 & 21734 & 96.094 & 2647 & 138.142 & 438 & 188.155 & 1048 & 259.242 & 2413 \\
\hline 55.115 & 453 & 97.067 & 1648 & 139.115 & 806 & 189.164 & 1919 & 260.214 & 1602 \\
\hline 56.064 & 3411 & 97.104 & 5127 & 141.073 & 1191 & 190.170 & 446 & 260.247 & 1683 \\
\hline 57.036 & 2606 & 98.075 & 1362 & 142.080 & 1347 & \begin{tabular}{|l}
191.156 \\
\end{tabular} & 508 & 261.225 & 1158 \\
\hline 57.073 & 13291 & 98.107 & 586 & 143.088 & 3655 & 192.151 & 648 & 261.254 & 1203 \\
\hline 58.044 & 402 & 99.081 & 388 & 144.096 & 1582 & 193.196 & 498 & 262.264 & 430 \\
\hline 58.076 & 697 & 99.121 & 308 & 145.103 & 7924 & 195.116 & 389 & 267.210 & 646 \\
\hline 59.052 & 456 & 103.057 & 1170 & 146.110 & 2494 & \begin{tabular}{|l|}
197.135 \\
\end{tabular} & 898 & 268.215 & 433 \\
\hline 60.023 & 1453 & 104.064 & 883 & 147.119 & 11326 & 198.142 & 333 & 269.227 & 18968 \\
\hline 61.031 & 551 & 105.072 & 16067 & 148.125 & 3162 & \begin{tabular}{|l|}
199.150 \\
\end{tabular} & 2645 & 269.362 & 793 \\
\hline 63.026 & 375 & 106.079 & 4276 & 149.134 & 4828 & 200.156 & 1155 & 270.229 & 4279 \\
\hline 65.041 & 1640 & 107.050 & 386 & \begin{tabular}{|l|l|}
150.139 \\
\end{tabular} & 797 & \begin{tabular}{|l|l|}
201.166 \\
\end{tabular} & 2470 & 271.216 & 1165 \\
\hline 66.049 & 790 & 107.088 & 12815 & 151.114 & 1275 & 202.171 & 545 & 273.249 & 830 \\
\hline 67.057 & 10625 & 107.178 & 411 & 151.149 & 755 & 203.180 & 863 & 274.267 & 734 \\
\hline 68.064 & 2662 & 108.095 & 4445 & 152.060 & 378 & 205.197 & 633 & 275.272 & 696 \\
\hline 69.037 & 485 & 109.069 & 575 & 152.157 & 530 & 206.205 & 372 & 281.225 & 334 \\
\hline 69.073 & 14149 & 109.103 & 9725 & 153.071 & 415 & 207.032 & 586 & 283.215 & 502 \\
\hline 69.141 & 371 & 110.109 & 1474 & 153.130 & 411 & 207.211 & 399 & 285.221 & 1916 \\
\hline 70.044 & 297 & 111.083 & 1253 & 154.080 & 474 & 209.141 & 287 & 286.227 & 565 \\
\hline 70.079 & 2166 & 111.119 & 1885 & 155.088 & 1241 & 211.150 & 779 & 287.237 & 3649 \\
\hline 71.052 & 1236 & 112.091 & 413 & 156.095 & 832 & 212.157 & 393 & 288.241 & 770 \\
\hline 71.088 & 5950 & 112.124 & 308 & 157.103 & 3885 & 213.165 & 4157 & 301.289 & 379 \\
\hline 72.092 & 310 & 113.099 & 403 & 158.110 & 1577 & \begin{tabular}{|l|l|}
214.172 \\
\end{tabular} & 1342 & 311.284 & 412 \\
\hline 73.031 & 1645 & 115.057 & 2148 & 159.119 & 7991 & 215.181 & 3065 & 313.288 & 706 \\
\hline 75.028 & 386 & 116.065 & 1065 & 160.125 & 2734 & 216.188 & 683 & 314.290 & 585 \\
\hline 77.042 & 4071 & 117.072 & 5192 & 161.135 & 10589 & \begin{tabular}{|l|l|}
217.182 \\
\end{tabular} & 564 & 315.300 & 560 \\
\hline 78.048 & 1835 & 118.080 & 2388 & 162.139 & 2073 & \begin{tabular}{|l|}
219.210 \\
\end{tabular} & 500 & 353.320 & 835 \\
\hline 79.057 & 10146 & 119.088 & 14053 & 163.116 & 619 & 225.167 & 576 & 365.313 & 373 \\
\hline 80.063 & 2049 & 120.095 & 5225 & 163.151 & 2179 & 227.181 & 11292 & 367.334 & 5589 \\
\hline 81.034 & 348 & 121.070 & 438 & 164.131 & 457 & 227.287 & 407 & 368.340 & 2053 \\
\hline 81.073 & 14492 & 121.104 & 12979 & 165.074 & 607 & 228.185 & 2751 & 369.339 & 620 \\
\hline 81.142 & 402 & 122.110 & 4177 & 165.129 & 1362 & 229.196 & 2337 & 371.338 & 398 \\
\hline 82.044 & 303 & 123.082 & 1081 & 167.087 & 441 & 230.195 & 505 & 382.357 & 2630 \\
\hline 82.080 & 4478 & 123.119 & 5221 & 168.101 & 354 & 231.176 & 1207 & 383.339 & 1542 \\
\hline 83.053 & 917 & 124.090 & 1035 & 169.104 & 855 & 232.185 & 819 & 384.337 & 593 \\
\hline 83.088 & 7800 & 124.124 & 702 & 170.110 & 493 & 233.192 & 616 & 385.345 & 8346 \\
\hline 84.060 & 1167 & 125.098 & 1549 & 171.119 & 3420 & 233.227 & 709 & 386.349 & 2926 \\
\hline 84.094 & 1020 & 125.137 & 638 & 172.125 & 1550 & 241.195 & 1574 & 387.356 & 542 \\
\hline 85.032 & 375 & 127.059 & 582 & \begin{tabular}{|l}
173.134 \\
\end{tabular} & 5696 & 242.203 & 1765 & 398.349 & 1191 \\
\hline 85.067 & 924 & 128.066 & 1634 & 174.141 & 2077 & 243.175 & 1059 & 399.352 & 614 \\
\hline 85.104 & 1875 & 129.072 & 3467 & 175.150 & 3479 & 243.211 & 5153 & 400.368 & 17126 \\
\hline 87.049 & 370 & 130.079 & 1332 & \begin{tabular}{|l}
176.153 \\
\end{tabular} & 620 & 244.214 & 1241 & 400.534 & 637 \\
\hline 89.047 & 299 & 131.088 & 6490 & 177.128 & 932 & 245.191 & 7597 & 401.372 & 5470 \\
\hline 91.057 & 13941 & 132.094 & 2710 & 177.165 & 825 & \begin{tabular}{|l|}
246.195 \\
\end{tabular} & 2056 & 402.375 & 830 \\
\hline 91.130 & 375 & 133.103 & 10407 & 178.141 & 381 & 247.241 & 2293 & & \\
\hline 92.064 & 3453 & 134.111 & 5668 & 179.145 & 444 & 248.255 & 427 & & \\
\hline
\end{tabular}


Table S28. High-resolution mass table for the compound identified as cholest-4-en-3-one.

\begin{tabular}{|c|c|c|c|c|c|c|c|c|c|}
\hline Mass & $\begin{array}{c}\text { Ion } \\
\text { counts }\end{array}$ & Mass & $\begin{array}{c}\text { Ion } \\
\text { counts }\end{array}$ & Mass & $\begin{array}{c}\text { Ion } \\
\text { counts }\end{array}$ & Mass & $\begin{array}{c}\text { Ion } \\
\text { counts }\end{array}$ & Mass & $\begin{array}{c}\text { Ion } \\
\text { counts }\end{array}$ \\
\hline 50.018 & 609 & 91.130 & 478 & 121.104 & 14448 & 149.098 & 7821 & 188.155 & 2326 \\
\hline 51.026 & 1071 & 91.177 & 409 & 121.175 & 406 & 149.135 & 9601 & 189.128 & 1191 \\
\hline 52.033 & 803 & 92.063 & 4468 & 122.075 & 8397 & 150.105 & 1885 & 189.166 & 2771 \\
\hline 53.041 & 5261 & 93.073 & 19816 & 122.110 & 3631 & 150.139 & 1475 & 190.171 & 840 \\
\hline 54.049 & 1991 & 93.157 & 507 & 123.083 & 11054 & 151.115 & 811 & 191.182 & 1315 \\
\hline 55.021 & 3701 & 94.045 & 512 & 123.120 & 6311 & 151.150 & 1242 & 192.186 & 492 \\
\hline 55.057 & 33256 & 94.080 & 6860 & 124.091 & 75049 & 152.064 & 218 & 193.197 & 1883 \\
\hline 55.118 & 701 & 95.052 & 2422 & 124.134 & 436 & 152.159 & 372 & 197.134 & 1834 \\
\hline 56.064 & 4813 & 95.088 & 25427 & 124.459 & 400 & 153.072 & 426 & 198.143 & 700 \\
\hline 57.037 & 1227 & 95.168 & 645 & 124.493 & 366 & 154.081 & 438 & 199.150 & 1690 \\
\hline 57.073 & 21779 & 96.059 & 2006 & 124.538 & 612 & 155.088 & 1688 & 200.156 & 1457 \\
\hline 57.133 & 495 & 96.094 & 3276 & 124.586 & 460 & 156.094 & 1199 & 201.130 & 1515 \\
\hline 58.045 & 387 & 97.067 & 3949 & 124.876 & 488 & 157.104 & 3166 & 201.165 & 3817 \\
\hline 58.076 & 1163 & 97.104 & 5550 & 124.933 & 480 & 158.110 & 1575 & 202.136 & 1283 \\
\hline 59.052 & 352 & 98.075 & 1430 & 125.095 & 8180 & 159.083 & 1676 & 202.171 & 2414 \\
\hline 60.023 & 1083 & 98.111 & 430 & 125.135 & 753 & 159.119 & 6835 & 203.143 & 941 \\
\hline 61.031 & 440 & 99.042 & 253 & 125.238 & 496 & 160.090 & 1219 & 203.181 & 1834 \\
\hline 63.025 & 392 & 99.083 & 471 & 126.096 & 738 & 160.125 & 2335 & 204.188 & 471 \\
\hline 65.042 & 3236 & 99.120 & 340 & 127.059 & 515 & 161.098 & 3798 & 205.197 & 1323 \\
\hline 66.049 & 1501 & 101.064 & 373 & 127.151 & 350 & 161.135 & 4126 & 206.204 & 1527 \\
\hline 67.021 & 1700 & 101.086 & 304 & 128.065 & 1974 & 162.105 & 2764 & 207.036 & 671 \\
\hline 67.057 & 17304 & 102.053 & 304 & 129.072 & 3822 & 162.141 & 917 & 207.209 & 691 \\
\hline 67.116 & 428 & 103.056 & 1792 & 130.081 & 1568 & 163.114 & 2132 & 209.142 & 350 \\
\hline 68.064 & 4316 & 104.065 & 1739 & 131.054 & 405 & 163.150 & 2732 & 211.150 & 5100 \\
\hline 69.036 & 876 & 105.038 & 376 & 131.088 & 8338 & 164.130 & 586 & 212.156 & 2512 \\
\hline 69.073 & 18612 & 105.073 & 18524 & 132.094 & 3069 & 165.072 & 400 & 213.165 & 3245 \\
\hline 70.079 & 2635 & 105.146 & 476 & 133.067 & 2091 & 165.167 & 1353 & 214.172 & 1167 \\
\hline 71.051 & 599 & 106.079 & 6865 & 133.104 & 13130 & 167.090 & 372 & 215.145 & 1791 \\
\hline 71.088 & 9322 & 107.052 & 3210 & 134.075 & 1784 & 169.104 & 1537 & 215.181 & 1957 \\
\hline 72.093 & 438 & 107.088 & 19230 & 134.111 & 8424 & 170.111 & 1181 & 216.153 & 981 \\
\hline 73.032 & 1559 & 107.166 & 531 & 135.083 & 2956 & 171.119 & 3002 & 216.184 & 797 \\
\hline 75.030 & 353 & 108.059 & 1610 & 135.119 & 17369 & 172.125 & 1092 & 217.160 & 1012 \\
\hline 77.041 & 9270 & 108.095 & 5851 & 135.212 & 566 & 173.099 & 1494 & 217.196 & 532 \\
\hline 78.049 & 3035 & 109.068 & 6340 & 136.091 & 3522 & 173.135 & 5076 & 219.212 & 1393 \\
\hline 79.057 & 21751 & 109.104 & 11931 & 136.124 & 3577 & 174.106 & 2768 & 220.217 & 444 \\
\hline 79.131 & 477 & 109.177 & 462 & 137.051 & 408 & 174.141 & 1944 & 225.166 & 1266 \\
\hline 80.063 & 3778 & 110.074 & 1919 & 137.099 & 8654 & 175.113 & 2527 & 226.174 & 1702 \\
\hline 81.036 & 1241 & 110.109 & 1664 & 137.135 & 1806 & 175.150 & 3194 & 227.180 & 2109 \\
\hline 81.073 & 21847 & 111.083 & 3722 & 138.105 & 2740 & 176.121 & 1906 & 228.187 & 1338 \\
\hline 81.141 & 593 & 111.119 & 2767 & 139.114 & 506 & 176.155 & 924 & 229.160 & 9659 \\
\hline 82.043 & 387 & 112.090 & 664 & 141.073 & 1489 & 177.128 & 1665 & 229.196 & 27735 \\
\hline 82.079 & 3933 & 113.087 & 396 & 142.080 & 1386 & 177.164 & 1614 & 229.298 & 1236 \\
\hline 83.053 & 1874 & 113.134 & 348 & 143.088 & 3520 & 178.131 & 507 & 230.167 & 4361 \\
\hline 83.088 & 9470 & 115.058 & 2448 & 144.095 & 1779 & 179.180 & 987 & 230.199 & 5946 \\
\hline 84.060 & 827 & 116.065 & 1409 & 145.068 & 785 & 181.106 & 359 & 231.175 & 3355 \\
\hline 84.094 & 1383 & 117.073 & 6133 & 145.103 & 7553 & 183.120 & 1507 & 232.182 & 823 \\
\hline 85.069 & 624 & 118.080 & 2585 & 146.111 & 3767 & 184.127 & 1192 & 232.284 & 359 \\
\hline 85.104 & 2299 & 119.088 & 13157 & 147.083 & 3009 & 185.135 & 3129 & 233.227 & 980 \\
\hline 87.050 & 220 & 119.179 & 420 & 147.119 & 18612 & 186.141 & 1513 & 236.242 & 311 \\
\hline 87.073 & 352 & 120.060 & 430 & 147.206 & 481 & 187.112 & 1392 & 239.187 & 481 \\
\hline 89.041 & 321 & 120.095 & 7692 & 148.090 & 8653 & 187.150 & 8086 & 241.199 & 405 \\
\hline 91.057 & 21435 & 121.067 & 5055 & 148.125 & 4672 & 188.124 & 1114 & 243.175 & 1878 \\
\hline
\end{tabular}


Table S28 (continued). High-resolution mass table for the compound identified as cholest-4-en-3-one.

\begin{tabular}{|c|c|c|c|c|c|c|c|c|c|}
\hline Mass & $\begin{array}{c}\text { Ion } \\
\text { counts }\end{array}$ & Mass & $\begin{array}{c}\text { Ion } \\
\text { counts }\end{array}$ & Mass & $\begin{array}{c}\text { Ion } \\
\text { counts }\end{array}$ & Mass & $\begin{array}{c}\text { Ion } \\
\text { counts }\end{array}$ & Mass & $\begin{array}{c}\text { Ion } \\
\text { counts }\end{array}$ \\
\hline 243.211 & 1320 & 259.242 & 1179 & 275.274 & 785 & 329.315 & 359 & 368.342 & 873 \\
\hline 244.183 & 3315 & 260.250 & 8857 & 281.230 & 520 & 340.309 & 787 & 369.314 & 6637 \\
\hline 245.191 & 2275 & 261.258 & 14385 & 285.246 & 558 & 341.314 & 1094 & 370.320 & 2117 \\
\hline 245.228 & 3810 & 261.385 & 522 & 286.265 & 2126 & 342.327 & 10718 & 371.322 & 421 \\
\hline 246.232 & 1495 & 262.262 & 3083 & 287.267 & 569 & 342.484 & 505 & 382.342 & 553 \\
\hline 247.243 & 5049 & 263.275 & 446 & 298.261 & 524 & 343.331 & 3166 & 383.327 & 517 \\
\hline 248.248 & 1271 & 267.195 & 385 & 299.273 & 7489 & 344.336 & 525 & 384.337 & 13634 \\
\hline 251.946 & 313 & 269.190 & 788 & 300.279 & 2848 & 351.304 & 1652 & 384.491 & 698 \\
\hline 253.196 & 2735 & 269.223 & 776 & 301.285 & 800 & 352.315 & 498 & 385.340 & 4383 \\
\hline 254.201 & 772 & 270.209 & 484 & 302.296 & 453 & 353.319 & 405 & 386.345 & 954 \\
\hline 255.209 & 857 & 271.206 & 5744 & 313.289 & 707 & 355.325 & 474 & 400.362 & 448 \\
\hline 256.198 & 374 & 272.208 & 1372 & 314.292 & 518 & 356.332 & 676 & \\
\hline 257.227 & 2548 & 273.259 & 755 & 327.304 & 5803 & 366.327 & 2306 & \\
\hline 258.234 & 684 & 274.265 & 651 & 328.307 & 1727 & 367.336 & 1538 &
\end{tabular}




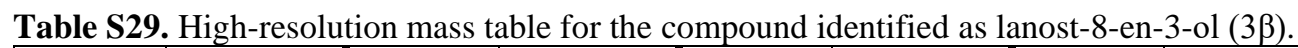

\begin{tabular}{|c|c|c|c|c|c|c|c|c|c|}
\hline Mass & $\begin{array}{c}\text { Ion } \\
\text { counts }\end{array}$ & Mass & $\begin{array}{c}\text { Ion } \\
\text { counts }\end{array}$ & Mass & $\begin{array}{c}\text { Ion } \\
\text { counts }\end{array}$ & Mass & $\begin{array}{c}\text { Ion } \\
\text { counts }\end{array}$ & Mass & $\begin{array}{c}\text { Ion } \\
\text { counts }\end{array}$ \\
\hline 50.018 & 551 & 73.067 & 502 & 103.057 & 2366 & \begin{tabular}{|l|l|}
127.149 \\
\end{tabular} & 479 & 159.215 & 739 \\
\hline 51.025 & 1230 & 75.027 & 356 & 104.065 & 1671 & 128.065 & 5257 & 160.125 & 7202 \\
\hline 52.033 & 738 & 76.034 & 269 & 105.072 & 44482 & 129.072 & 10813 & 161.099 & 972 \\
\hline 53.041 & 7124 & 77.041 & 10174 & 105.157 & 1210 & \begin{tabular}{|l|l|}
130.079 \\
\end{tabular} & 4960 & 161.135 & 35870 \\
\hline 54.049 & 2121 & 78.048 & 2670 & 105.472 & 425 & 131.088 & 22527 & 161.240 & 1008 \\
\hline 55.021 & 3051 & 79.057 & 24943 & 106.078 & 7604 & \begin{tabular}{|l|l|}
131.153 \\
\end{tabular} & 319 & 162.139 & 6588 \\
\hline 55.057 & 80104 & 79.108 & 292 & 106.164 & 418 & \begin{tabular}{|l|l|}
131.186 \\
\end{tabular} & 514 & 163.114 & 1260 \\
\hline 55.325 & 614 & 79.135 & 592 & 106.217 & 337 & 132.094 & 5343 & 163.150 & 13726 \\
\hline 55.350 & 530 & 80.063 & 3642 & 107.052 & 628 & 133.104 & 33388 & 163.287 & 424 \\
\hline 55.580 & 528 & 81.036 & 445 & 107.088 & 46542 & 133.194 & 933 & 164.155 & 2421 \\
\hline 55.611 & 436 & 81.073 & 43811 & 107.499 & 459 & 134.110 & 8355 & 165.073 & 1133 \\
\hline 55.827 & 424 & 81.147 & 1208 & 108.094 & 7611 & 135.083 & 1101 & 165.129 & 1648 \\
\hline 56.027 & 531 & 82.045 & 419 & 108.167 & 494 & 135.119 & 41782 & 165.165 & 3270 \\
\hline 56.063 & 7921 & 82.079 & 5891 & 109.067 & 1438 & 136.125 & 8091 & 166.081 & 781 \\
\hline 56.109 & 426 & 83.052 & 2522 & 109.104 & 46917 & 137.043 & 399 & 166.169 & 678 \\
\hline 57.036 & 8072 & 83.088 & 54240 & 109.489 & 515 & 137.098 & 1785 & 167.087 & 1647 \\
\hline 57.073 & 67704 & 83.163 & 1562 & 109.827 & 444 & 137.135 & 10128 & 167.144 & 573 \\
\hline 57.316 & 420 & 83.418 & 445 & 110.075 & 468 & 138.107 & 562 & 168.095 & 1357 \\
\hline 57.345 & 323 & 83.466 & 482 & 110.108 & 5197 & 138.140 & 1669 & 169.103 & 4187 \\
\hline 57.367 & 501 & 84.059 & 1370 & 110.176 & 423 & 139.114 & 2886 & 170.111 & 2355 \\
\hline 57.399 & 470 & 84.092 & 4537 & 111.083 & 5056 & \begin{tabular}{|c|}
139.150 \\
\end{tabular} & 924 & 171.119 & 12030 \\
\hline 57.580 & 413 & 85.067 & 3723 & 111.119 & 22031 & 140.120 & 785 & 171.211 & 380 \\
\hline 57.611 & 445 & 85.104 & 8280 & 111.206 & 612 & \begin{tabular}{|l|}
141.072 \\
\end{tabular} & 3761 & 172.125 & 4289 \\
\hline 58.043 & 908 & 86.069 & 542 & 111.540 & 376 & 141.124 & 397 & 173.134 & 23308 \\
\hline 58.076 & 3272 & 86.108 & 523 & 112.087 & 579 & 142.080 & 4610 & 173.245 & 599 \\
\hline 59.052 & 2559 & 87.047 & 456 & 112.124 & 2254 & \begin{tabular}{|l|}
143.088 \\
\end{tabular} & 12087 & 174.106 & 1039 \\
\hline 60.024 & 1534 & 89.043 & 292 & 113.098 & 1111 & \begin{tabular}{|l|}
144.094 \\
\end{tabular} & 5318 & \begin{tabular}{|l|l|}
1749 \\
\end{tabular} & 5657 \\
\hline 61.031 & 635 & 91.057 & 31612 & 113.135 & 1327 & 145.103 & 28350 & 175.114 & 821 \\
\hline 63.025 & 451 & 92.063 & 5029 & 114.098 & 348 & \begin{tabular}{|l|}
146.109 \\
\end{tabular} & 6548 & 175.150 & 22198 \\
\hline 65.042 & 3458 & 93.073 & 39660 & 115.057 & 5090 & \begin{tabular}{|l|}
147.086 \\
\end{tabular} & 866 & 175.259 & 595 \\
\hline 66.048 & 1182 & 93.123 & 445 & 116.064 & 2745 & 147.119 & 32820 & 176.153 & 4101 \\
\hline 67.057 & 26862 & 93.157 & 950 & 117.072 & 12061 & \begin{tabular}{|l}
147.216 \\
\end{tabular} & 1066 & 177.131 & 1185 \\
\hline 67.104 & 447 & 93.197 & 457 & 117.142 & 388 & \begin{tabular}{|l|}
148.124 \\
\end{tabular} & 6149 & \begin{tabular}{|l|l|}
177.165 \\
\end{tabular} & 4894 \\
\hline 67.128 & 402 & 93.421 & 214 & 118.079 & 3778 & \begin{tabular}{|c|}
149.099 \\
\end{tabular} & 1039 & 178.080 & 318 \\
\hline 68.064 & 4535 & 94.078 & 7049 & 119.088 & 51022 & \begin{tabular}{|l|}
149.135 \\
\end{tabular} & 21714 & 178.170 & 951 \\
\hline 69.036 & 1303 & 95.053 & 740 & 119.483 & 320 & \begin{tabular}{|l|}
149.226 \\
\end{tabular} & 636 & 179.085 & 643 \\
\hline 69.073 & 94733 & 95.088 & 80617 & 119.531 & 472 & \begin{tabular}{|l|}
150.139 \\
\end{tabular} & 4479 & 179.144 & 1833 \\
\hline 69.374 & 409 & 95.164 & 2324 & 120.094 & 9683 & \begin{tabular}{|l|}
151.114 \\
\end{tabular} & 1647 & 179.181 & 3478 \\
\hline 69.402 & 565 & 95.449 & 618 & 121.068 & 1125 & \begin{tabular}{|l|}
151.150 \\
\end{tabular} & 5002 & 180.093 & 504 \\
\hline 69.428 & 558 & 95.487 & 340 & 121.104 & 47275 & 152.065 & 695 & 180.184 & 736 \\
\hline 69.648 & 519 & 95.768 & 476 & 122.109 & 9076 & \begin{tabular}{|l|}
152.120 \\
\end{tabular} & 759 & 181.103 & 1351 \\
\hline 69.677 & 583 & 96.058 & 634 & 123.083 & 2238 & \begin{tabular}{|l|}
152.155 \\
\end{tabular} & 875 & 182.111 & 948 \\
\hline 69.941 & 429 & 96.093 & 8329 & 123.119 & 24664 & \begin{tabular}{|l|}
153.074 \\
\end{tabular} & 1623 & 183.119 & 3897 \\
\hline 70.044 & 433 & 97.067 & 3158 & 123.213 & 646 & \begin{tabular}{|l|}
153.130 \\
\end{tabular} & 4310 & 184.126 & 1734 \\
\hline 70.077 & 7429 & 97.104 & 36571 & 124.091 & 822 & \begin{tabular}{|l|}
153.167 \\
\end{tabular} & 660 & 185.135 & 10661 \\
\hline 70.177 & 423 & 97.186 & 966 & 124.123 & 2891 & 154.079 & 1520 & 186.141 & 4785 \\
\hline 71.052 & 7054 & 98.075 & 1937 & 125.098 & 3102 & 154.135 & 684 & 187.150 & 26860 \\
\hline 71.088 & 33705 & 98.108 & 3521 & 125.135 & 6665 & 155.088 & 4686 & 188.154 & 5684 \\
\hline 71.134 & 367 & 99.082 & 1745 & 126.102 & 440 & 156.095 & 3507 & 189.131 & 1018 \\
\hline 72.056 & 821 & 99.120 & 1390 & 126.140 & 755 & 157.104 & 12198 & 189.165 & 18563 \\
\hline 72.092 & 2149 & 100.083 & 467 & 127.057 & 1468 & \begin{tabular}{|l|l}
158.109 \\
\end{tabular} & 4170 & 190.170 & 3526 \\
\hline 73.031 & 1858 & 101.062 & 405 & 127.114 & 1061 & 159.119 & 26919 & 191.145 & 2293 \\
\hline
\end{tabular}


Table S29 (continued). High-resolution mass table for the compound identified as lanost-8-en-3-ol (3ß).

\begin{tabular}{|c|c|c|c|c|c|c|c|c|c|}
\hline Mass & $\begin{array}{c}\text { Ion } \\
\text { counts }\end{array}$ & Mass & $\begin{array}{c}\text { Ion } \\
\text { counts }\end{array}$ & Mass & $\begin{array}{c}\text { Ion } \\
\text { counts }\end{array}$ & Mass & $\begin{array}{c}\text { Ion } \\
\text { counts }\end{array}$ & Mass & $\begin{array}{c}\text { Ion } \\
\text { counts }\end{array}$ \\
\hline 191.181 & 4173 & 223.151 & 524 & 255.333 & 568 & 288.278 & 2655 & 368.341 & 832 \\
\hline 192.182 & 947 & 224.158 & 515 & 256.216 & 3494 & 289.290 & 1462 & 369.349 & 1010 \\
\hline 193.161 & 1464 & 225.165 & 2727 & 257.192 & 2441 & 295.245 & 947 & 370.346 & 496 \\
\hline 193.197 & 6123 & 226.172 & 1883 & 257.226 & 4940 & 296.246 & 348 & 371.341 & 480 \\
\hline 194.113 & 337 & 227.181 & 18237 & 258.231 & 1609 & 297.258 & 3884 & 379.332 & 976 \\
\hline 194.200 & 1257 & 227.297 & 585 & 259.206 & 7485 & 298.263 & 1244 & 380.341 & 2006 \\
\hline 195.118 & 1031 & 228.185 & 4849 & 259.241 & 8182 & 299.239 & 3548 & 381.347 & 940 \\
\hline 196.127 & 657 & 228.307 & 393 & 259.347 & 504 & 299.270 & 6014 & 382.340 & 603 \\
\hline 197.135 & 3531 & 229.196 & 19713 & 260.213 & 1661 & 300.263 & 2344 & 386.350 & 511 \\
\hline 198.139 & 2207 & 229.306 & 664 & 260.248 & 2466 & 301.288 & 5115 & 393.348 & 1760 \\
\hline 199.150 & 10071 & 230.199 & 4115 & 261.221 & 1811 & 302.290 & 1387 & 394.355 & 454 \\
\hline 200.155 & 3173 & 231.177 & 2928 & 261.258 & 9273 & 303.262 & 389 & 395.150 & 628 \\
\hline 201.165 & 14203 & 231.209 & 2918 & 261.413 & 457 & 309.258 & 1017 & 395.365 & 157481 \\
\hline 201.297 & 480 & 232.194 & 1381 & 262.260 & 2101 & 310.269 & 527 & 396.029 & 832 \\
\hline 202.170 & 3133 & 233.191 & 2814 & 265.195 & 421 & 311.273 & 2203 & 396.086 & 864 \\
\hline 203.148 & 1021 & 233.227 & 3874 & 266.203 & 1770 & 312.281 & 897 & 396.164 & 1900 \\
\hline 203.181 & 7007 & 234.218 & 1182 & 267.211 & 2700 & 313.288 & 3959 & 396.369 & 49488 \\
\hline 204.184 & 1996 & 235.243 & 523 & 268.217 & 965 & 314.292 & 1231 & 396.756 & 1171 \\
\hline 205.161 & 2991 & 237.163 & 613 & 269.227 & 5150 & 315.270 & 2450 & 396.920 & 530 \\
\hline 205.196 & 5447 & 238.175 & 461 & 270.233 & 2213 & 316.269 & 643 & 397.372 & 9236 \\
\hline 206.166 & 831 & 239.181 & 2512 & 271.209 & 1841 & 323.272 & 383 & 397.916 & 3056 \\
\hline 206.203 & 2138 & 240.188 & 2097 & 271.241 & 3483 & 325.288 & 3760 & 398.017 & 1750 \\
\hline 207.035 & 742 & 241.196 & 20150 & 272.211 & 519 & 326.293 & 1357 & 398.374 & 911 \\
\hline 207.177 & 1155 & 241.316 & 656 & 272.248 & 976 & 327.303 & 3431 & 398.879 & 751 \\
\hline 207.212 & 4760 & 242.201 & 4718 & 273.221 & 5778 & 328.309 & 1203 & 399.359 & 564 \\
\hline 208.214 & 1251 & 243.211 & 11736 & 273.257 & 20121 & 329.309 & 1117 & 409.367 & 419 \\
\hline 209.132 & 809 & 243.800 & 396 & 273.392 & 841 & 337.287 & 2010 & 410.389 & 2151 \\
\hline 210.139 & 494 & 244.216 & 2580 & 274.226 & 1724 & 338.292 & 484 & 411.360 & 10220 \\
\hline 211.150 & 3124 & 245.192 & 8886 & 274.263 & 6457 & 339.304 & 2879 & 412.365 & 3470 \\
\hline 212.157 & 2297 & 246.194 & 1578 & 274.359 & 356 & 340.309 & 1063 & 413.375 & 130429 \\
\hline 213.165 & 13011 & 246.228 & 933 & 275.273 & 8818 & 341.320 & 2722 & 414.209 & 1685 \\
\hline 214.172 & 9941 & 247.206 & 5976 & 275.396 & 313 & 342.326 & 1093 & 414.379 & 41485 \\
\hline 215.181 & 24200 & 247.241 & 4605 & 275.452 & 453 & 343.300 & 1182 & 414.784 & 871 \\
\hline 215.299 & 790 & 248.210 & 1174 & 276.277 & 2338 & 344.300 & 440 & 414.913 & 673 \\
\hline 216.184 & 4602 & 248.243 & 975 & 279.206 & 387 & 351.300 & 370 & 415.382 & 7490 \\
\hline 217.196 & 4145 & 249.259 & 987 & 281.226 & 3592 & 352.310 & 975 & 415.510 & 806 \\
\hline 218.202 & 890 & 251.182 & 731 & 282.234 & 1728 & 353.321 & 2481 & 416.388 & 946 \\
\hline 219.175 & 1787 & 251.948 & 275 & 283.242 & 7468 & 354.323 & 992 & 426.386 & 1046 \\
\hline 219.211 & 7135 & 252.189 & 594 & 284.245 & 2197 & 355.297 & 2654 & 427.391 & 381 \\
\hline 220.216 & 2057 & 253.196 & 1862 & 285.257 & 4053 & 356.304 & 561 & 428.398 & 13827 \\
\hline 221.227 & 2026 & 254.203 & 712 & 286.262 & 1265 & 365.319 & 592 & 429.402 & 4734 \\
\hline 222.230 & 467 & 255.212 & 13899 & 287.272 & 4807 & 367.334 & 2152 & 430.407 & 755 \\
\hline
\end{tabular}


Table S30. High-resolution mass table for the compound identified as lanosterol.

\begin{tabular}{|c|c|c|c|c|c|c|c|}
\hline Mass & $\begin{array}{c}\text { Ion } \\
\text { counts }\end{array}$ & Mass & $\begin{array}{c}\text { Ion } \\
\text { counts }\end{array}$ & Mass & $\begin{array}{c}\text { Ion } \\
\text { counts }\end{array}$ & Mass & $\begin{array}{c}\text { Ion } \\
\text { counts }\end{array}$ \\
\hline 50.018 & 270 & 92.063 & 2546 & 131.088 & 8538 & 171.119 & 4827 \\
\hline 51.026 & 819 & 93.073 & 16322 & 132.095 & 2274 & 172.126 & 1763 \\
\hline 52.034 & 498 & 94.045 & 382 & \begin{tabular}{|l|}
133.104 \\
\end{tabular} & 12492 & 173.135 & 8435 \\
\hline 53.041 & 4451 & 94.079 & 3072 & 134.110 & 3527 & 174.140 & 2443 \\
\hline 54.049 & 1395 & 95.088 & 28911 & \begin{tabular}{|l|}
135.119 \\
\end{tabular} & 15353 & 175.150 & 7446 \\
\hline 55.021 & 1854 & 96.059 & 344 & 135.200 & 444 & 176.153 & 1549 \\
\hline 55.057 & 33481 & 96.093 & 3541 & \begin{tabular}{|l|}
136.124 \\
\end{tabular} & 2868 & 177.166 & 2850 \\
\hline 56.029 & 382 & 97.068 & 1496 & \begin{tabular}{|l|}
137.047 \\
\end{tabular} & 332 & 178.168 & 410 \\
\hline 56.063 & 3726 & 97.104 & 5832 & \begin{tabular}{|c|}
137.099 \\
\end{tabular} & 766 & 179.086 & 338 \\
\hline 57.037 & 2968 & 98.074 & 1328 & 137.135 & 5928 & 179.149 & 538 \\
\hline 57.073 & 8514 & 98.108 & 729 & 137.231 & 290 & 179.179 & 471 \\
\hline 58.042 & 262 & 99.082 & 773 & 138.140 & 1100 & 181.103 & 681 \\
\hline 58.076 & 291 & 101.057 & 357 & 139.113 & 877 & 182.111 & 373 \\
\hline 59.052 & 1339 & 103.056 & 1262 & 140.119 & 225 & 183.119 & 1674 \\
\hline 60.024 & 1342 & 104.064 & 856 & \begin{tabular}{|l|}
141.073 \\
\end{tabular} & 1974 & 184.127 & 692 \\
\hline 61.030 & 556 & 105.073 & 17054 & 142.080 & 2157 & 185.135 & 4756 \\
\hline 63.027 & 305 & 105.156 & 493 & 143.088 & 5008 & 186.141 & 2052 \\
\hline 65.042 & 1937 & 106.078 & 2962 & \begin{tabular}{|l|}
144.094 \\
\end{tabular} & 2372 & 187.150 & 10137 \\
\hline 66.049 & 727 & 107.088 & 17878 & 145.104 & 11018 & 188.155 & 2590 \\
\hline 67.057 & 13964 & 108.095 & 3103 & \begin{tabular}{|l|l|}
146.110 \\
\end{tabular} & 2526 & 189.166 & 9716 \\
\hline 67.119 & 423 & 109.067 & 704 & \begin{tabular}{|l|l|}
147.120 \\
\end{tabular} & 11358 & 190.170 & 2369 \\
\hline 68.064 & 2772 & 109.104 & 34719 & 148.125 & 2615 & 191.146 & 975 \\
\hline 69.037 & 491 & 109.190 & 1052 & \begin{tabular}{|l|}
149.098 \\
\end{tabular} & 606 & 191.181 & 3152 \\
\hline 69.073 & 74503 & 109.522 & 409 & 149.135 & 9262 & 192.185 & 636 \\
\hline 69.374 & 377 & 110.108 & 3671 & 150.138 & 1430 & 193.162 & 558 \\
\hline 69.401 & 559 & 111.083 & 2040 & 151.114 & 512 & 195.117 & 599 \\
\hline 69.447 & 327 & 111.119 & 4310 & 151.149 & 1613 & 196.126 & 356 \\
\hline 69.681 & 531 & 112.088 & 433 & 152.064 & 475 & 197.134 & 1698 \\
\hline 70.044 & 312 & 112.125 & 405 & 152.144 & 427 & 198.143 & 931 \\
\hline 70.077 & 5352 & 113.100 & 361 & 153.071 & 980 & 199.150 & 4153 \\
\hline 71.052 & 2953 & 115.057 & 2308 & \begin{tabular}{|l|l|}
153.130 \\
\end{tabular} & 830 & 200.156 & 1203 \\
\hline 71.088 & 3441 & 116.065 & 1486 & 154.079 & 841 & 201.165 & 5951 \\
\hline 72.056 & 354 & 117.073 & 5379 & 155.088 & 2092 & 202.169 & 1637 \\
\hline 73.031 & 1395 & 118.079 & 1631 & 156.095 & 1549 & 203.181 & 4816 \\
\hline 73.068 & 381 & 119.088 & 18411 & 157.104 & 4975 & 204.186 & 1066 \\
\hline 75.028 & 338 & 119.170 & 457 & \begin{tabular}{|l|l}
158.109 \\
\end{tabular} & 1884 & 205.161 & 1333 \\
\hline 77.042 & 5345 & 120.094 & 3678 & 159.119 & 10358 & 205.196 & 2000 \\
\hline 78.049 & 1738 & 121.104 & 16899 & 160.125 & 3502 & 206.202 & 779 \\
\hline 79.057 & 11467 & 121.187 & 420 & 161.135 & 10993 & 207.034 & 718 \\
\hline 80.063 & 1830 & 122.109 & 3329 & 162.139 & 2200 & 207.198 & 457 \\
\hline 81.073 & 23229 & 123.084 & 893 & 163.115 & 520 & 209.136 & 491 \\
\hline 81.147 & 501 & 123.120 & 12082 & \begin{tabular}{|l|}
163.150 \\
\end{tabular} & 4726 & 211.150 & 1755 \\
\hline 82.079 & 4231 & 123.220 & 403 & 164.155 & 940 & 212.155 & 928 \\
\hline 83.052 & 1284 & 124.089 & 533 & \begin{tabular}{|l|}
165.074 \\
\end{tabular} & 807 & 213.165 & 5056 \\
\hline 83.088 & 12605 & 124.124 & 1389 & \begin{tabular}{|l|}
165.132 \\
\end{tabular} & 482 & 214.173 & 3311 \\
\hline 83.158 & 456 & 125.099 & 1285 & 165.165 & 1862 & 215.181 & 8417 \\
\hline 84.059 & 726 & 125.134 & 725 & \begin{tabular}{|l|}
166.080 \\
\end{tabular} & 379 & 216.186 & 1791 \\
\hline 84.094 & 1476 & 127.058 & 610 & \begin{tabular}{|l}
166.166 \\
\end{tabular} & 357 & 217.195 & 2604 \\
\hline 85.067 & 1344 & 127.113 & 408 & \begin{tabular}{|l|}
167.089 \\
\end{tabular} & 592 & 218.199 & 474 \\
\hline 85.104 & 1578 & 128.065 & 2534 & \begin{tabular}{|l|}
168.092 \\
\end{tabular} & 517 & 219.211 & 848 \\
\hline 87.046 & 439 & 129.073 & 4784 & \begin{tabular}{|l|}
169.103 \\
\end{tabular} & 2136 & 223.151 & 305 \\
\hline 91.057 & 13809 & 130.079 & 2278 & \begin{tabular}{|l|}
170.109 \\
\end{tabular} & 1030 & 224.149 & 434 \\
\hline
\end{tabular}


Table S30 (continued). High-resolution mass table for the compound identified as lanosterol.

\begin{tabular}{|c|c|c|c|c|c|c|c|}
\hline Mass & $\begin{array}{c}\text { Ion } \\
\text { counts }\end{array}$ & Mass & $\begin{array}{c}\text { Ion } \\
\text { counts }\end{array}$ & Mass & $\begin{array}{c}\text { Ion } \\
\text { counts }\end{array}$ & Mass & $\begin{array}{c}\text { Ion } \\
\text { counts }\end{array}$ \\
\hline 225.166 & 1340 & 257.192 & 1511 & 295.244 & 1154 & 350.299 & 362 \\
\hline 226.171 & 804 & 257.226 & 3423 & 296.258 & 424 & 351.311 & 408 \\
\hline 227.181 & 5884 & 258.235 & 969 & 297.258 & 2091 & 353.322 & 438 \\
\hline 228.187 & 1748 & 259.207 & 6214 & 298.262 & 579 & 355.298 & 723 \\
\hline 229.196 & 7466 & 260.211 & 1267 & 299.238 & 920 & 365.319 & 440 \\
\hline 230.201 & 1623 & 261.220 & 716 & 299.270 & 1137 & 378.323 & 789 \\
\hline 231.176 & 1182 & 267.211 & 1231 & 300.240 & 490 & 379.332 & 397 \\
\hline 231.209 & 1239 & 268.219 & 387 & 301.255 & 557 & 391.329 & 450 \\
\hline 232.182 & 442 & 269.226 & 2280 & 309.257 & 1922 & 393.351 & 32995 \\
\hline 233.191 & 895 & 270.230 & 778 & 310.263 & 523 & 394.353 & 10764 \\
\hline 239.181 & 1493 & 271.207 & 932 & 311.274 & 2685 & 395.357 & 2527 \\
\hline 240.190 & 957 & 271.242 & 3485 & 312.278 & 746 & 396.015 & 517 \\
\hline 241.196 & 9169 & 272.247 & 1502 & 313.254 & 877 & 396.365 & 512 \\
\hline 242.200 & 2375 & 273.221 & 2271 & 314.261 & 955 & 408.377 & 828 \\
\hline 243.212 & 4437 & 273.253 & 1489 & 315.271 & 701 & 409.347 & 3321 \\
\hline 244.214 & 1063 & 274.227 & 674 & 323.274 & 1669 & 410.356 & 1058 \\
\hline 245.191 & 3197 & 275.270 & 388 & 324.280 & 616 & 411.360 & 38233 \\
\hline 246.198 & 797 & 279.204 & 262 & 325.288 & 1622 & 412.364 & 12296 \\
\hline 247.207 & 2197 & 281.226 & 1697 & 326.296 & 618 & 413.367 & 2504 \\
\hline 248.216 & 408 & 282.237 & 585 & 327.270 & 901 & 414.368 & 449 \\
\hline 251.178 & 520 & 283.243 & 2580 & 329.287 & 549 & 424.370 & 603 \\
\hline 252.190 & 269 & 284.250 & 511 & 337.290 & 1072 & 426.383 & 7900 \\
\hline 253.198 & 958 & 285.256 & 1207 & 339.304 & 1414 & 427.389 & 2868 \\
\hline 254.203 & 295 & 286.266 & 553 & 340.306 & 495 & 428.394 & 469 \\
\hline 255.212 & 6098 & 287.233 & 577 & 341.285 & 2711 & 939.603 & 349 \\
\hline 256.216 & 1648 & 288.246 & 549 & 342.289 & 877 & & \\
\hline & & & & & & & \\
\hline
\end{tabular}


Table S31. High-resolution mass table for the compound identified as hexadecenoic acid hexadecyl ester.

\begin{tabular}{|c|c|c|c|c|c|c|c|c|c|}
\hline Mass & $\begin{array}{l}\text { Ion } \\
\text { counts }\end{array}$ & Mass & $\begin{array}{c}\text { Ion } \\
\text { counts }\end{array}$ & Mass & $\begin{array}{c}\text { Ion } \\
\text { counts }\end{array}$ & Mass & $\begin{array}{c}\text { Ion } \\
\text { counts }\end{array}$ & Mass & $\begin{array}{c}\text { Ion } \\
\text { counts }\end{array}$ \\
\hline 49.008 & 259 & 57.457 & 507 & 68.065 & 163166 & 72.853 & 393 & 83.418 & 3078 \\
\hline 50.018 & 1396 & 57.611 & 7879 & 68.364 & 1171 & 73.031 & 60928 & 83.446 & 4668 \\
\hline 51.026 & 2746 & 57.863 & 5796 & 68.426 & 1711 & 73.068 & 8911 & 83.481 & 4458 \\
\hline 52.034 & 2259 & 58.044 & 5829 & 68.474 & 494 & 73.096 & 2965 & 83.518 & 811 \\
\hline 53.042 & 30168 & 58.076 & 45816 & 68.668 & 1545 & 73.312 & 579 & 83.540 & 1015 \\
\hline 53.099 & 708 & 58.117 & 4908 & 68.917 & 1281 & 73.340 & 690 & 83.736 & 4297 \\
\hline 53.332 & 340 & 58.146 & 3756 & 68.951 & 1433 & 73.369 & 748 & 84.023 & 3496 \\
\hline 54.014 & 371 & 58.350 & 3457 & 68.993 & 1460 & 73.602 & 524 & 84.060 & 400108 \\
\hline 54.049 & 121357 & 58.381 & 3134 & 69.037 & 8376 & 73.647 & 642 & 84.096 & 152272 \\
\hline 54.109 & 3336 & 58.555 & 1499 & 69.073 & 609081 & 73.930 & 499 & 84.139 & 11463 \\
\hline 54.289 & 609 & 58.607 & 2445 & 69.314 & 1797 & 74.038 & 6124 & 84.361 & 3687 \\
\hline 54.315 & 685 & 58.841 & 1901 & 69.353 & 3083 & 74.072 & 392 & 84.391 & 3021 \\
\hline 54.342 & 982 & 58.872 & 1476 & 69.374 & 3706 & 74.158 & 420 & 84.409 & 2003 \\
\hline 54.394 & 202 & 58.894 & 1069 & 69.411 & 6312 & 74.192 & 386 & 84.427 & 4681 \\
\hline 54.572 & 644 & 59.052 & 7336 & 69.464 & 1410 & 74.508 & 345 & 84.490 & 773 \\
\hline 54.603 & 552 & 59.080 & 2196 & 69.481 & 1030 & 75.038 & 1420 & 84.514 & 646 \\
\hline 54.784 & 591 & 59.117 & 1387 & 69.491 & 984 & 76.040 & 423 & 84.703 & 3605 \\
\hline 54.823 & 770 & 59.322 & 807 & 69.670 & 5258 & 77.042 & 11785 & 84.771 & 2065 \\
\hline 54.866 & 689 & 59.354 & 844 & 69.926 & 3642 & 77.098 & 338 & 84.979 & 2279 \\
\hline 55.021 & 29831 & 59.375 & 770 & 69.960 & 3614 & 78.049 & 6379 & 85.032 & 8244 \\
\hline 55.058 & 644249 & 59.610 & 689 & 69.996 & 2222 & 79.057 & 67073 & 85.066 & 55415 \\
\hline 55.096 & 37747 & 59.636 & 341 & 70.044 & 8527 & 79.340 & 795 & 85.104 & 357583 \\
\hline 55.273 & 3692 & 59.659 & 380 & 70.080 & 225724 & 79.377 & 554 & 85.152 & 3113 \\
\hline 55.311 & 3379 & 59.826 & 504 & 70.355 & 537 & 79.422 & 365 & 85.405 & 2258 \\
\hline 55.326 & 5068 & 59.858 & 519 & 70.383 & 1137 & 79.456 & 438 & 85.438 & 1937 \\
\hline 55.360 & 7445 & 60.024 & 22455 & 70.439 & 2594 & 79.663 & 418 & 85.468 & 2447 \\
\hline 55.407 & 2236 & 60.057 & 790 & 70.467 & 1951 & 80.065 & 64793 & 85.505 & 2324 \\
\hline 55.422 & 718 & 60.322 & 424 & 70.495 & 1921 & 80.388 & 435 & 85.560 & 767 \\
\hline 55.431 & 381 & 60.370 & 430 & 70.679 & 1647 & 80.450 & 305 & 85.757 & 2023 \\
\hline 55.589 & 6098 & 60.583 & 427 & 70.713 & 1987 & 80.740 & 358 & 85.792 & 1829 \\
\hline 55.617 & 5076 & 61.031 & 20218 & 70.748 & 1660 & 81.037 & 3995 & 85.984 & 396 \\
\hline 55.807 & 4232 & 61.322 & 364 & 70.973 & 1538 & 81.073 & 268154 & 86.039 & 5779 \\
\hline 55.848 & 4339 & 61.849 & 388 & 71.019 & 1768 & 81.370 & 1697 & 86.071 & 5054 \\
\hline 56.028 & 6248 & 62.034 & 745 & 71.052 & 31472 & 81.398 & 1595 & 86.108 & 23250 \\
\hline 56.065 & 236933 & 63.027 & 812 & 71.089 & 545564 & 81.433 & 2783 & 86.151 & 721 \\
\hline 56.092 & 995 & 65.042 & 9770 & 71.133 & 7521 & 81.518 & 604 & 86.183 & 529 \\
\hline 56.104 & 3366 & 66.049 & 13907 & 71.366 & 2787 & 81.709 & 2139 & 86.304 & 405 \\
\hline 56.309 & 3711 & 66.108 & 402 & 71.393 & 2659 & 81.749 & 1896 & 86.361 & 700 \\
\hline 56.336 & 4220 & 67.038 & 331 & 71.424 & 4517 & 81.943 & 645 & 86.419 & 726 \\
\hline 56.572 & 2920 & 67.057 & 239015 & 71.484 & 1183 & 81.998 & 1606 & 86.648 & 503 \\
\hline 56.605 & 2793 & 67.101 & 1907 & 71.503 & 1023 & 82.044 & 10158 & 86.686 & 500 \\
\hline 56.806 & 2305 & 67.326 & 1408 & 71.695 & 3770 & 82.081 & 224038 & 86.727 & 374 \\
\hline 56.823 & 1379 & 67.353 & 1582 & 71.967 & 2621 & 82.375 & 1782 & 87.002 & 424 \\
\hline 56.846 & 2533 & 67.382 & 2185 & 71.998 & 1597 & 82.408 & 1431 & 87.047 & 46326 \\
\hline 56.938 & 308 & 67.467 & 425 & 72.024 & 1132 & 82.442 & 1929 & 87.084 & 4384 \\
\hline 56.990 & 1122 & 67.601 & 1462 & 72.057 & 3191 & 82.534 & 494 & 87.112 & 1799 \\
\hline 57.037 & 24071 & 67.650 & 1958 & 72.092 & 30130 & 82.732 & 1570 & 87.354 & 365 \\
\hline 57.074 & 781697 & 67.682 & 1728 & 72.213 & 1787 & 83.052 & 67421 & 87.383 & 429 \\
\hline 57.113 & 61456 & 67.879 & 1306 & 72.249 & 1923 & 83.089 & 542556 & 88.054 & 5292 \\
\hline 57.346 & 7640 & 67.924 & 1483 & 72.517 & 1516 & 83.165 & 14862 & 89.061 & 1445 \\
\hline 57.382 & 9889 & 67.961 & 941 & 72.746 & 449 & 83.340 & 829 & 91.057 & 20964 \\
\hline 57.428 & 4268 & 68.028 & 2473 & 72.780 & 749 & 83.390 & 3715 & 91.134 & 517 \\
\hline
\end{tabular}


Table S31 (continued). High-resolution mass table for the compound identified as hexadecenoic acid hexadecyl ester.

\begin{tabular}{|c|c|c|c|c|c|c|c|c|c|}
\hline Mass & $\begin{array}{c}\text { Ion } \\
\text { counts }\end{array}$ & Mass & $\begin{array}{c}\text { Ion } \\
\text { counts }\end{array}$ & Mass & $\begin{array}{c}\text { Ion } \\
\text { counts }\end{array}$ & Mass & $\begin{array}{c}\text { Ion } \\
\text { counts }\end{array}$ & Mass & $\begin{array}{c}\text { Ion } \\
\text { counts }\end{array}$ \\
\hline 92.064 & 7070 & 98.766 & 4004 & 110.168 & 5613 & 116.085 & 11720 & 125.653 & 557 \\
\hline 93.073 & 46057 & 99.047 & 11995 & 110.461 & 1721 & 116.176 & 577 & 125.689 & 339 \\
\hline 93.156 & 1130 & 99.082 & 39271 & 110.492 & 1263 & 117.073 & 4835 & 125.944 & 918 \\
\hline 93.425 & 288 & 99.120 & 69078 & 110.530 & 2178 & 118.080 & 3804 & 126.004 & 1078 \\
\hline 93.451 & 423 & 99.170 & 3898 & 110.837 & 1911 & 119.089 & 36542 & 126.071 & 9960 \\
\hline 94.045 & 2661 & 99.446 & 2905 & 110.883 & 1700 & 119.173 & 1013 & 126.106 & 14785 \\
\hline 94.081 & 52185 & 99.489 & 2488 & 111.049 & 1067 & 119.519 & 425 & 126.143 & 41183 \\
\hline 94.383 & 432 & 99.755 & 2050 & 111.083 & 98969 & 119.599 & 365 & 126.235 & 2085 \\
\hline 94.436 & 257 & 99.798 & 1991 & 111.120 & 203657 & 120.095 & 12607 & 126.283 & 1180 \\
\hline 94.464 & 391 & 100.055 & 34412 & 111.205 & 8473 & 120.196 & 474 & 126.352 & 498 \\
\hline 94.499 & 493 & 100.087 & 3368 & 111.468 & 2387 & 121.068 & 2981 & 126.608 & 486 \\
\hline 95.052 & 16963 & 100.123 & 7034 & 111.506 & 2824 & 121.104 & 50104 & 126.664 & 258 \\
\hline 95.089 & 214687 & 100.388 & 1100 & 111.547 & 3341 & 121.468 & 424 & 127.078 & 81997 \\
\hline 95.139 & 1950 & 100.432 & 1552 & 111.861 & 3034 & 121.510 & 380 & 127.115 & 12331 \\
\hline 95.413 & 1533 & 100.500 & 610 & 112.055 & 2543 & 121.556 & 471 & 127.151 & 20940 \\
\hline 95.441 & 1288 & 100.720 & 910 & 112.091 & 47855 & 121.595 & 469 & 127.203 & 2207 \\
\hline 95.471 & 2274 & 100.760 & 1032 & 112.127 & 65788 & 121.868 & 444 & 127.293 & 458 \\
\hline 95.573 & 266 & 100.818 & 586 & 112.202 & 5097 & 122.076 & 3781 & 127.485 & 568 \\
\hline 95.768 & 1745 & 101.063 & 67354 & 112.511 & 2048 & 122.111 & 19895 & 127.530 & 578 \\
\hline 95.811 & 1719 & 101.100 & 2906 & 112.570 & 2438 & 122.197 & 823 & 127.568 & 586 \\
\hline 96.029 & 1212 & 101.395 & 941 & 112.883 & 2018 & 123.084 & 111688 & 127.848 & 448 \\
\hline 96.060 & 205784 & 101.427 & 451 & 112.932 & 1730 & 123.120 & 64990 & 127.904 & 573 \\
\hline 96.096 & 167873 & 101.455 & 836 & 113.063 & 36348 & 123.181 & 4533 & 128.085 & 23059 \\
\hline 96.148 & 8856 & 101.505 & 534 & 113.099 & 19815 & 123.485 & 1099 & 128.121 & 1672 \\
\hline 96.388 & 2341 & 101.708 & 422 & 113.135 & 34553 & 123.533 & 1369 & 128.155 & 2707 \\
\hline 96.415 & 3327 & 101.760 & 510 & 113.185 & 2070 & 123.566 & 1035 & 128.239 & 632 \\
\hline 96.451 & 4391 & 101.799 & 529 & 113.222 & 3216 & 123.602 & 768 & 129.094 & 35557 \\
\hline 96.518 & 1336 & 102.069 & 9186 & 113.524 & 1403 & 123.823 & 508 & 129.132 & 1570 \\
\hline 96.544 & 1231 & 102.109 & 486 & 113.562 & 701 & 123.870 & 1020 & 129.186 & 840 \\
\hline 96.778 & 3529 & 102.151 & 516 & 113.594 & 1302 & 123.919 & 1381 & 130.100 & 8584 \\
\hline 97.034 & 3526 & 103.075 & 2607 & 113.886 & 1457 & 123.983 & 436 & 131.089 & 5295 \\
\hline 97.068 & 155101 & 104.065 & 1455 & 113.929 & 1060 & 124.091 & 58344 & 132.096 & 2519 \\
\hline 97.104 & 439266 & 105.073 & 15179 & 114.071 & 85646 & 124.128 & 93808 & 133.104 & 38815 \\
\hline 97.183 & 14669 & 106.080 & 6597 & 114.107 & 1303 & 124.450 & 263 & 133.149 & 551 \\
\hline 97.379 & 1736 & 107.053 & 1156 & 114.140 & 5383 & 124.494 & 921 & \begin{tabular}{|l|}
133.199 \\
\end{tabular} & 1183 \\
\hline 97.428 & 5321 & 107.089 & 34538 & 114.218 & 1869 & 124.532 & 816 & 134.112 & 32774 \\
\hline 97.461 & 3527 & 107.167 & 1102 & 114.458 & 311 & 124.571 & 1953 & 135.084 & 3815 \\
\hline 97.478 & 2236 & 108.060 & 1183 & 114.485 & 454 & 124.620 & 1547 & 135.120 & 46426 \\
\hline 97.506 & 5521 & 108.096 & 32695 & 114.533 & 724 & 124.679 & 463 & 135.953 & 437 \\
\hline 97.554 & 2001 & 108.180 & 951 & 114.605 & 590 & 124.904 & 1047 & 136.091 & 5017 \\
\hline 97.591 & 466 & 108.227 & 422 & 114.809 & 384 & 124.941 & 1119 & 136.127 & 15580 \\
\hline 97.771 & 4675 & 108.892 & 407 & 114.843 & 362 & 124.985 & 1114 & 136.218 & 497 \\
\hline 97.815 & 4436 & 109.068 & 26810 & 114.886 & 583 & 125.063 & 1454 & 136.279 & 460 \\
\hline 98.040 & 2265 & 109.104 & 111993 & 114.927 & 637 & 125.099 & 60466 & 137.041 & 470 \\
\hline 98.076 & 271299 & 109.190 & 3840 & 115.078 & 48715 & 125.135 & 93165 & 137.099 & 80149 \\
\hline 98.111 & 102331 & 109.455 & 730 & 115.115 & 3060 & 125.193 & 2653 & 137.136 & 35006 \\
\hline 98.160 & 10757 & 109.496 & 1362 & 115.167 & 2008 & 125.227 & 4412 & 137.198 & 3155 \\
\hline 98.406 & 3648 & 109.828 & 818 & 115.470 & 382 & 125.503 & 582 & 137.522 & 731 \\
\hline 98.434 & 3430 & 109.872 & 970 & 115.509 & 445 & 125.539 & 725 & 137.564 & 577 \\
\hline 98.468 & 5313 & 110.076 & 125930 & 115.545 & 441 & 125.574 & 848 & 137.609 & 532 \\
\hline 98.565 & 630 & 110.112 & 83704 & 115.898 & 443 & 125.610 & 1415 & 137.649 & 402 \\
\hline
\end{tabular}


Table S31 (continued). High-resolution mass table for the compound identified as hexadecenoic acid hexadecyl ester.

\begin{tabular}{|c|c|c|c|c|c|c|c|c|c|}
\hline Mass & $\begin{array}{c}\text { Ion } \\
\text { counts }\end{array}$ & Mass & $\begin{array}{c}\text { Ion } \\
\text { counts }\end{array}$ & Mass & $\begin{array}{c}\text { Ion } \\
\text { counts }\end{array}$ & Mass & $\begin{array}{c}\text { Ion } \\
\text { counts }\end{array}$ & Mass & $\begin{array}{c}\text { Ion } \\
\text { counts }\end{array}$ \\
\hline 137.898 & 607 & 151.267 & 1317 & 167.146 & 19436 & 192.691 & 477 & 213.173 & 7688 \\
\hline 137.948 & 821 & 151.562 & 653 & 167.180 & 19338 & 193.162 & 24497 & 214.183 & 2059 \\
\hline 138.040 & 256 & 151.648 & 587 & 167.246 & 1111 & 193.196 & 20072 & 215.195 & 2207 \\
\hline 138.106 & 49751 & 151.982 & 595 & 167.602 & 465 & 193.273 & 1382 & 216.188 & 526 \\
\hline 138.143 & 52555 & 152.059 & 1335 & 168.118 & 2544 & 194.169 & 14007 & 217.198 & 25459 \\
\hline 138.205 & 2084 & 152.122 & 48172 & 168.151 & 3122 & 194.206 & 112519 & 217.316 & 878 \\
\hline 138.246 & 2565 & 152.159 & 109626 & 168.189 & 8300 & 194.311 & 4066 & 218.205 & 23975 \\
\hline 138.364 & 375 & 152.225 & 2398 & 169.125 & 12037 & 194.649 & 802 & 218.321 & 856 \\
\hline 138.607 & 900 & 152.500 & 454 & 169.161 & 3830 & 194.719 & 1112 & 219.213 & 31646 \\
\hline 138.664 & 760 & 152.569 & 752 & 169.197 & 5124 & 194.788 & 715 & 219.743 & 536 \\
\hline 138.954 & 595 & 152.613 & 863 & 170.131 & 4225 & 195.143 & 1851 & 219.836 & 511 \\
\hline 139.017 & 649 & 152.649 & 781 & 170.200 & 642 & 195.176 & 7800 & 220.219 & 11199 \\
\hline 139.077 & 1510 & 152.707 & 867 & 171.141 & 15414 & 195.210 & 22061 & 220.343 & 581 \\
\hline 139.114 & 46665 & 153.093 & 2577 & 172.148 & 6977 & 195.276 & 1244 & 221.193 & 8217 \\
\hline 139.151 & 31693 & 153.130 & 35213 & 173.142 & 4000 & 195.620 & 481 & 221.225 & 3450 \\
\hline 139.216 & 2146 & 153.165 & 31263 & 174.143 & 1034 & 195.715 & 509 & 221.756 & 615 \\
\hline 139.625 & 469 & 154.101 & 2934 & 175.151 & 11177 & 196.149 & 1803 & 221.817 & 690 \\
\hline 140.086 & 8310 & 154.135 & 6038 & 175.254 & 433 & 196.180 & 1581 & 222.200 & 11005 \\
\hline 140.121 & 7512 & 154.174 & 15918 & 176.157 & 4125 & 196.221 & 9632 & 222.236 & 11216 \\
\hline 140.159 & 21996 & 155.110 & 16191 & 177.132 & 1224 & 197.156 & 5678 & 223.207 & 5238 \\
\hline 140.214 & 463 & 155.146 & 11980 & 177.167 & 6741 & 197.227 & 2450 & 223.240 & 3260 \\
\hline 140.248 & 805 & 155.182 & 7481 & 178.083 & 357 & 198.163 & 2526 & 224.180 & 4858 \\
\hline 141.094 & 25657 & 155.244 & 701 & 178.138 & 1417 & 199.171 & 7043 & 224.253 & 8834 \\
\hline 141.130 & 21942 & 156.116 & 5284 & 178.174 & 5408 & 200.176 & 2352 & 224.332 & 526 \\
\hline 141.166 & 12083 & 156.151 & 1578 & 179.146 & 33990 & 201.187 & 7321 & 225.188 & 5138 \\
\hline 141.244 & 1144 & 156.186 & 796 & 179.182 & 5626 & 202.190 & 1131 & 225.258 & 3895 \\
\hline 141.299 & 458 & 157.125 & 17079 & 179.250 & 1306 & 203.183 & 2967 & 226.195 & 6898 \\
\hline 141.599 & 486 & 157.232 & 501 & 180.153 & 14765 & 204.190 & 1849 & 226.260 & 707 \\
\hline 142.036 & 370 & 158.132 & 11460 & 180.190 & 19092 & 205.198 & 3330 & 227.203 & 25210 \\
\hline 142.101 & 8856 & 159.121 & 6062 & 180.273 & 768 & 206.170 & 911 & 227.304 & 796 \\
\hline 142.134 & 2603 & 160.127 & 2288 & 181.126 & 1805 & 206.205 & 2385 & 228.206 & 5717 \\
\hline 142.172 & 1394 & 161.136 & 23001 & 181.161 & 11728 & 207.038 & 632 & 229.165 & 994 \\
\hline 143.109 & 22685 & 161.241 & 600 & 181.196 & 6577 & 207.177 & 32022 & 229.218 & 8018 \\
\hline 143.147 & 1625 & 162.142 & 7428 & 182.133 & 1975 & 207.293 & 1126 & 230.221 & 1487 \\
\hline 143.203 & 626 & 163.115 & 1768 & 182.167 & 2586 & 208.185 & 102467 & 231.177 & 2126 \\
\hline 144.117 & 8064 & 163.151 & 14798 & 182.205 & 6165 & 208.225 & 5246 & 232.182 & 480 \\
\hline 145.104 & 7591 & 163.230 & 515 & 183.141 & 8884 & 208.296 & 4299 & 232.220 & 484 \\
\hline 146.111 & 2140 & 164.123 & 1896 & 183.177 & 4013 & 208.710 & 1163 & 233.229 & 1052 \\
\hline 147.120 & 30149 & 164.159 & 32253 & 183.212 & 3260 & 208.751 & 743 & 234.201 & 1795 \\
\hline 147.214 & 928 & 165.073 & 973 & 184.147 & 3738 & 208.816 & 726 & 235.208 & 28183 \\
\hline 148.127 & 23214 & 165.130 & 50117 & 185.156 & 10196 & 209.191 & 41849 & 235.331 & 764 \\
\hline 148.218 & 620 & 165.166 & 20281 & 186.164 & 5746 & 209.388 & 533 & 236.216 & 218043 \\
\hline 149.099 & 2672 & 165.236 & 2108 & 187.161 & 3523 & 209.665 & 787 & 236.719 & 1248 \\
\hline 149.135 & 26313 & 165.633 & 378 & 188.163 & 550 & 209.756 & 750 & 236.777 & 2210 \\
\hline 149.197 & 431 & 166.137 & 23690 & 189.167 & 21409 & 210.196 & 6549 & 236.855 & 2691 \\
\hline 149.228 & 576 & 166.174 & 84318 & 189.247 & 652 & 210.235 & 2443 & 236.974 & 524 \\
\hline 150.107 & 4506 & 166.596 & 551 & 190.174 & 16671 & 210.707 & 506 & 237.222 & 86313 \\
\hline 150.142 & 9382 & 166.640 & 335 & 190.354 & 402 & 211.172 & 6772 & 237.343 & 5272 \\
\hline 151.115 & 68270 & 166.704 & 575 & 191.182 & 22542 & 211.207 & 4762 & 237.792 & 2444 \\
\hline 151.151 & 20489 & 166.788 & 455 & 192.110 & 463 & 211.586 & 412 & 238.226 & 14448 \\
\hline 151.216 & 2744 & 167.109 & 2195 & 192.190 & 55619 & 212.178 & 2772 & 238.340 & 2523 \\
\hline
\end{tabular}


Table S31 (continued). High-resolution mass table for the compound identified as hexadecenoic acid hexadecyl ester.

\begin{tabular}{|c|c|c|c|c|c|c|c|c|c|}
\hline Mass & $\begin{array}{c}\text { Ion } \\
\text { counts }\end{array}$ & Mass & $\begin{array}{c}\text { Ion } \\
\text { counts }\end{array}$ & Mass & $\begin{array}{c}\text { Ion } \\
\text { counts }\end{array}$ & Mass & $\begin{array}{c}\text { Ion } \\
\text { counts }\end{array}$ & Mass & $\begin{array}{c}\text { Ion } \\
\text { counts }\end{array}$ \\
\hline 238.792 & 1506 & 252.211 & 6983 & 271.209 & 474 & 302.295 & 834 & 365.342 & 902 \\
\hline 238.849 & 1593 & 252.283 & 2441 & 271.261 & 381 & 305.287 & 822 & 366.343 & 403 \\
\hline 239.236 & 4934 & 253.218 & 4100 & 273.224 & 1405 & 307.303 & 1277 & 367.340 & 361 \\
\hline 239.423 & 703 & 253.290 & 1273 & 274.268 & 964 & 308.309 & 521 & 368.346 & 3353 \\
\hline 239.756 & 761 & 254.226 & 9437 & 275.275 & 4434 & 309.283 & 729 & 369.348 & 1295 \\
\hline 239.832 & 1107 & 255.234 & 43350 & 276.280 & 1129 & 311.289 & 655 & 371.331 & 2178 \\
\hline 240.213 & 1960 & 255.352 & 1686 & 277.277 & 684 & 313.300 & 370 & 372.333 & 652 \\
\hline 240.325 & 676 & 256.238 & 8181 & 278.298 & 2106 & 314.295 & 370 & 379.356 & 1495 \\
\hline 240.861 & 558 & 256.355 & 567 & 279.270 & 2740 & 319.311 & 377 & 380.360 & 625 \\
\hline 241.219 & 4428 & 257.249 & 5889 & 280.244 & 1394 & 321.319 & 520 & 381.367 & 498 \\
\hline 241.342 & 605 & 258.252 & 1029 & 281.251 & 1219 & 322.322 & 408 & 382.373 & 481 \\
\hline 242.223 & 1391 & 259.245 & 366 & 282.257 & 1403 & 323.297 & 1575 & 384.338 & 648 \\
\hline 243.233 & 3297 & 260.249 & 534 & 283.264 & 4228 & 324.296 & 333 & 386.356 & 2589 \\
\hline 244.236 & 855 & 261.258 & 539 & 284.271 & 1425 & 325.310 & 1174 & 387.360 & 754 \\
\hline 245.229 & 3238 & 262.232 & 730 & 285.279 & 756 & 326.303 & 961 & 393.377 & 431 \\
\hline 246.236 & 3236 & 263.240 & 4369 & 291.269 & 445 & 327.307 & 552 & 395.393 & 481 \\
\hline 247.244 & 4513 & 264.247 & 30386 & 292.284 & 464 & 329.318 & 443 & 396.398 & 710 \\
\hline 248.248 & 1077 & 265.253 & 17091 & 293.287 & 581 & 337.311 & 1387 & 407.406 & 407 \\
\hline 249.226 & 1596 & 265.381 & 776 & 294.297 & 521 & 338.320 & 497 & 410.412 & 618 \\
\hline 249.946 & 364 & 265.929 & 471 & 295.266 & 1902 & 339.321 & 637 & 435.419 & 887 \\
\hline 250.231 & 3730 & 266.257 & 4091 & 296.271 & 615 & 350.354 & 352 & 436.431 & 334 \\
\hline 250.267 & 3550 & 267.255 & 1702 & 297.281 & 4075 & 351.326 & 2906 & 478.473 & 3590 \\
\hline 251.239 & 2857 & 268.240 & 783 & 298.283 & 941 & 352.334 & 918 & 479.480 & 1981 \\
\hline 251.269 & 1570 & 269.250 & 6352 & 299.273 & 327 & 353.324 & 4592 & 480.489 & 414 \\
\hline 251.943 & 372 & 270.253 & 1420 & 301.291 & 2997 & 354.325 & 1413 & & \\
\hline
\end{tabular}


Table S32. High-resolution mass table for the compound identified as $\mathrm{C}_{34} \mathrm{H}_{66} \mathrm{O}_{2}$.

\begin{tabular}{|c|c|c|c|c|c|c|c|c|c|}
\hline Mass & $\begin{array}{c}\text { Ion } \\
\text { counts }\end{array}$ & Mass & $\begin{array}{c}\text { Ion } \\
\text { counts }\end{array}$ & Mass & $\begin{array}{c}\text { Ion } \\
\text { counts }\end{array}$ & Mass & $\begin{array}{c}\text { Ion } \\
\text { counts }\end{array}$ & Mass & $\begin{array}{c}\text { Ion } \\
\text { counts }\end{array}$ \\
\hline 50.018 & 1512 & 57.845 & 5502 & 68.028 & 2299 & 72.221 & 1868 & 83.389 & 3960 \\
\hline 51.026 & 2838 & 57.876 & 5166 & 68.065 & 182436 & 72.275 & 1598 & 83.418 & 3177 \\
\hline 52.034 & 1922 & 58.044 & 5413 & 68.108 & 1588 & 72.530 & 1467 & 83.451 & 5722 \\
\hline 53.042 & 26617 & 58.076 & 45066 & 68.336 & 837 & 72.772 & 770 & 83.517 & 992 \\
\hline 53.085 & 497 & 58.109 & 3190 & 68.363 & 1160 & 72.793 & 741 & 83.539 & 1005 \\
\hline 54.012 & 387 & 58.135 & 4677 & 68.393 & 1890 & 72.839 & 485 & 83.754 & 4347 \\
\hline 54.049 & 127456 & 58.329 & 2876 & 68.429 & 1877 & 73.031 & 60826 & 84.023 & 3676 \\
\hline 54.089 & 1271 & 58.357 & 3375 & 68.470 & 577 & 73.068 & 8797 & 84.060 & 360203 \\
\hline 54.288 & 701 & 58.390 & 2804 & 68.651 & 1456 & 73.318 & 596 & 84.095 & 145506 \\
\hline 54.315 & 884 & 58.585 & 2204 & 68.685 & 1721 & 73.343 & 890 & 84.139 & 10545 \\
\hline 54.339 & 856 & 58.616 & 2400 & 68.897 & 1115 & 73.614 & 521 & 84.360 & 3543 \\
\hline 54.358 & 596 & 58.837 & 1725 & 68.918 & 767 & 73.651 & 663 & 84.391 & 2656 \\
\hline 54.384 & 561 & 58.869 & 1764 & 68.948 & 1549 & 73.692 & 411 & 84.418 & 4196 \\
\hline 54.557 & 695 & 59.052 & 6744 & 68.996 & 874 & 73.920 & 485 & 84.453 & 3401 \\
\hline 54.592 & 705 & 59.080 & 1830 & 69.037 & 7867 & 74.038 & 6121 & 84.513 & 626 \\
\hline 54.779 & 552 & 59.107 & 1456 & 69.073 & 606174 & 74.077 & 234 & 84.724 & 3397 \\
\hline 54.814 & 769 & 59.134 & 941 & 69.316 & 1587 & 74.185 & 403 & 84.985 & 2357 \\
\hline 54.835 & 878 & 59.159 & 554 & 69.354 & 3069 & 75.036 & 1461 & 85.031 & 7322 \\
\hline 54.893 & 453 & 59.311 & 671 & 69.374 & 3639 & 76.036 & 408 & 85.066 & 48727 \\
\hline 54.950 & 469 & 59.357 & 1001 & 69.409 & 6255 & 77.042 & 9080 & 85.104 & 345327 \\
\hline 55.021 & 26163 & 59.387 & 605 & 69.464 & 1355 & 78.049 & 5986 & 85.153 & 2986 \\
\hline 55.057 & 628853 & 59.551 & 372 & 69.481 & 1734 & 79.057 & 60794 & 85.406 & 1976 \\
\hline 55.095 & 32908 & 59.597 & 711 & 69.656 & 5030 & 79.336 & 776 & 85.437 & 1742 \\
\hline 55.273 & 3360 & 59.625 & 581 & 69.919 & 3465 & 79.381 & 609 & 85.470 & 2699 \\
\hline 55.310 & 3439 & 59.801 & 396 & 69.955 & 3647 & 79.443 & 586 & 85.524 & 1635 \\
\hline 55.326 & 4665 & 59.841 & 454 & 70.044 & 7235 & 79.662 & 420 & 85.561 & 730 \\
\hline 55.359 & 7200 & 59.882 & 381 & 70.080 & 216006 & 80.065 & 63135 & 85.746 & 2021 \\
\hline 55.401 & 3068 & 60.024 & 24009 & 70.357 & 414 & 80.395 & 606 & 85.797 & 1631 \\
\hline 55.422 & 867 & 60.057 & 554 & 70.383 & 995 & 81.037 & 3464 & 86.039 & 5185 \\
\hline 55.431 & 643 & 60.308 & 402 & 70.444 & 2537 & 81.073 & 286300 & 86.070 & 4593 \\
\hline 55.585 & 6023 & 60.560 & 389 & 70.471 & 1673 & 81.121 & 2027 & 86.108 & 22964 \\
\hline 55.826 & 4429 & 60.609 & 389 & 70.492 & 1796 & 81.369 & 1762 & 86.155 & 591 \\
\hline 56.028 & 6043 & 61.031 & 19705 & 70.700 & 1978 & 81.398 & 1583 & 86.179 & 660 \\
\hline 56.065 & 225800 & 62.034 & 578 & 70.745 & 1692 & 81.430 & 2675 & 86.315 & 428 \\
\hline 56.092 & 920 & 63.026 & 620 & 70.962 & 1358 & 81.468 & 2150 & 86.366 & 788 \\
\hline 56.104 & 3171 & 65.042 & 8322 & 70.989 & 1222 & 81.519 & 702 & 86.430 & 563 \\
\hline 56.124 & 7647 & 66.049 & 15210 & 71.017 & 1359 & 81.710 & 2184 & 86.640 & 491 \\
\hline 56.310 & 3684 & 66.103 & 445 & 71.052 & 27772 & 81.746 & 1989 & 86.693 & 475 \\
\hline 56.335 & 2492 & 67.038 & 433 & 71.089 & 536390 & 81.783 & 1092 & 86.731 & 418 \\
\hline 56.357 & 2996 & 67.057 & 250119 & 71.161 & 12785 & 82.003 & 1611 & 86.964 & 406 \\
\hline 56.382 & 2312 & 67.328 & 1735 & 71.323 & 689 & 82.044 & 9091 & 87.047 & 43811 \\
\hline 56.574 & 2724 & 67.353 & 1673 & 71.366 & 2824 & 82.080 & 289783 & 87.083 & 3658 \\
\hline 56.814 & 2492 & 67.380 & 2067 & 71.393 & 2710 & 82.323 & 676 & 87.113 & 1684 \\
\hline 56.964 & 487 & 67.413 & 2100 & 71.409 & 1540 & 82.375 & 1852 & 88.053 & 5158 \\
\hline 57.036 & 21103 & 67.440 & 629 & 71.431 & 4422 & 82.408 & 1657 & 89.062 & 2160 \\
\hline 57.074 & 764582 & 67.461 & 426 & 71.485 & 962 & 82.438 & 2294 & 91.057 & 15722 \\
\hline 57.113 & 59662 & 67.596 & 1204 & 71.506 & 1141 & 82.531 & 616 & 91.125 & 495 \\
\hline 57.346 & 7376 & 67.638 & 1966 & 71.702 & 3640 & 82.680 & 1340 & 92.064 & 5307 \\
\hline 57.382 & 9625 & 67.671 & 1992 & 71.941 & 2397 & 82.725 & 1749 & 93.073 & 39254 \\
\hline 57.426 & 3819 & 67.874 & 1187 & 71.974 & 2632 & 83.052 & 62404 & 93.426 & 447 \\
\hline 57.446 & 1619 & 67.902 & 1523 & 72.057 & 2976 & 83.089 & 565020 & 94.045 & 2576 \\
\hline 57.611 & 7709 & 67.940 & 1338 & 72.092 & 29742 & 83.167 & 14696 & 94.081 & 50144 \\
\hline
\end{tabular}


Table S32 (continued). High-resolution mass table for the compound identified as $\mathrm{C}_{34} \mathrm{H}_{66} \mathrm{O}_{2}$.

\begin{tabular}{|c|c|c|c|c|c|c|c|c|c|}
\hline Mass & $\begin{array}{c}\text { Ion } \\
\text { counts }\end{array}$ & Mass & $\begin{array}{c}\text { Ion } \\
\text { counts }\end{array}$ & Mass & $\begin{array}{c}\text { Ion } \\
\text { counts }\end{array}$ & Mass & $\begin{array}{c}\text { Ion } \\
\text { counts }\end{array}$ & Mass & $\begin{array}{c}\text { Ion } \\
\text { counts }\end{array}$ \\
\hline 94.431 & 259 & 99.170 & 3821 & 110.455 & 1605 & 116.181 & 474 & 127.006 & 524 \\
\hline 94.472 & 428 & 99.438 & 2859 & 110.493 & 1979 & 117.076 & 1936 & 127.078 & 75757 \\
\hline 95.052 & 15207 & 99.484 & 2327 & 110.526 & 2353 & 117.091 & 1985 & 127.115 & 9370 \\
\hline 95.088 & 236782 & 99.744 & 1676 & 110.592 & 913 & 118.081 & 2596 & 127.151 & 23285 \\
\hline 95.408 & 1670 & 99.769 & 1249 & 110.861 & 1982 & 119.089 & 28382 & 127.196 & 1727 \\
\hline 95.441 & 1277 & 99.796 & 1888 & 111.083 & 90044 & 119.177 & 599 & 127.240 & 1555 \\
\hline 95.476 & 2362 & 99.860 & 592 & 111.120 & 212589 & 120.095 & 8971 & 127.305 & 542 \\
\hline 95.545 & 514 & 100.055 & 29979 & 111.174 & 4220 & 121.068 & 2756 & 127.503 & 704 \\
\hline 95.570 & 333 & 100.088 & 3391 & 111.206 & 8496 & 121.104 & 44068 & 127.604 & 504 \\
\hline 95.765 & 1875 & 100.123 & 7314 & 111.469 & 2349 & 121.189 & 1548 & 127.851 & 408 \\
\hline 95.814 & 1900 & 100.340 & 453 & 111.501 & 1729 & 121.241 & 698 & 127.889 & 382 \\
\hline 96.026 & 1397 & 100.381 & 742 & 111.532 & 3405 & 121.470 & 415 & 127.935 & 436 \\
\hline 96.060 & 185670 & 100.408 & 1041 & 111.582 & 2224 & 121.526 & 515 & 127.973 & 372 \\
\hline 96.096 & 235922 & 100.438 & 749 & 111.640 & 303 & 121.565 & 416 & 128.085 & 21217 \\
\hline 96.146 & 7071 & 100.471 & 1159 & 111.845 & 2571 & 122.076 & 3297 & 128.122 & 1054 \\
\hline 96.176 & 9448 & 100.684 & 401 & 111.891 & 2800 & 122.111 & 19694 & 128.154 & 2678 \\
\hline 96.391 & 2349 & 100.746 & 986 & 112.055 & 2499 & 122.208 & 681 & 128.229 & 509 \\
\hline 96.421 & 3344 & 100.803 & 469 & 112.091 & 42905 & 123.083 & 98019 & 129.094 & 34789 \\
\hline 96.451 & 4983 & 100.992 & 361 & 112.127 & 65502 & 123.120 & 72563 & 129.132 & 1298 \\
\hline 96.540 & 1466 & 101.063 & 61728 & 112.194 & 4966 & 123.184 & 4352 & 130.099 & 5954 \\
\hline 96.579 & 309 & 101.100 & 3004 & 112.479 & 1263 & 123.453 & 549 & 131.089 & 2604 \\
\hline 96.747 & 3588 & 101.387 & 609 & 112.516 & 1686 & 123.485 & 737 & 132.095 & 1428 \\
\hline 96.786 & 3791 & 101.424 & 452 & 112.567 & 2525 & 123.533 & 1543 & 133.061 & 397 \\
\hline 97.033 & 3238 & 101.449 & 707 & 112.860 & 1753 & 123.597 & 788 & 133.104 & 31644 \\
\hline 97.068 & 142336 & 101.506 & 417 & 112.905 & 1947 & 123.901 & 1135 & 133.201 & 964 \\
\hline 97.104 & 468292 & 101.737 & 448 & 113.063 & 33647 & 123.967 & 605 & 134.112 & 29198 \\
\hline 97.184 & 15224 & 101.785 & 460 & 113.098 & 12242 & 124.091 & 51797 & 135.084 & 3460 \\
\hline 97.369 & 1152 & 102.069 & 8650 & 113.135 & 38224 & 124.127 & 75891 & 135.120 & 42414 \\
\hline 97.429 & 5577 & 102.109 & 374 & 113.214 & 3265 & 124.181 & 2085 & 136.091 & 4498 \\
\hline 97.460 & 3917 & 102.141 & 395 & 113.286 & 1129 & 124.219 & 3818 & 136.127 & 16557 \\
\hline 97.492 & 6258 & 103.076 & 3110 & 113.485 & 687 & 124.495 & 682 & 136.222 & 448 \\
\hline 97.554 & 2076 & 104.066 & 902 & 113.541 & 1485 & 124.546 & 1607 & 137.047 & 641 \\
\hline 97.591 & 509 & 105.073 & 9351 & 113.878 & 1300 & 124.610 & 1411 & 137.099 & 70804 \\
\hline 97.792 & 5231 & 106.080 & 4674 & 113.910 & 773 & 124.903 & 947 & 137.136 & 45147 \\
\hline 97.875 & 1598 & 107.051 & 1048 & 113.943 & 948 & 124.946 & 1073 & 137.197 & 2786 \\
\hline 98.040 & 2084 & 107.089 & 26876 & 114.070 & 74576 & 125.008 & 761 & 137.522 & 612 \\
\hline 98.075 & 248514 & 107.139 & 354 & 114.108 & 572 & 125.063 & 1103 & 137.561 & 556 \\
\hline 98.111 & 99526 & 108.060 & 887 & 114.139 & 5565 & 125.099 & 54590 & 137.606 & 678 \\
\hline 98.160 & 10845 & 108.096 & 28993 & 114.221 & 1343 & 125.135 & 89781 & 137.923 & 670 \\
\hline 98.404 & 3635 & 109.068 & 23979 & 114.460 & 613 & 125.198 & 2996 & 137.966 & 753 \\
\hline 98.434 & 3069 & 109.104 & 123389 & 114.500 & 430 & 125.231 & 3758 & 138.017 & 410 \\
\hline 98.451 & 1842 & 109.158 & 1593 & 114.534 & 636 & 125.503 & 540 & 138.106 & 45236 \\
\hline 98.469 & 2773 & 109.444 & 836 & 114.597 & 539 & 125.547 & 1192 & 138.143 & 65005 \\
\hline 98.499 & 4389 & 109.482 & 934 & 114.839 & 405 & 125.598 & 1433 & 138.220 & 3321 \\
\hline 98.538 & 1069 & 109.522 & 1423 & 114.865 & 296 & 125.926 & 859 & 138.531 & 505 \\
\hline 98.566 & 686 & 109.564 & 884 & 114.911 & 481 & 125.961 & 859 & 138.591 & 838 \\
\hline 98.753 & 3677 & 109.793 & 606 & 114.951 & 363 & 126.071 & 8879 & 138.665 & 567 \\
\hline 98.791 & 3170 & 109.849 & 1133 & 115.078 & 46841 & 126.106 & 12792 & 138.976 & 592 \\
\hline 98.822 & 3025 & 109.924 & 546 & 115.116 & 3035 & 126.142 & 40731 & 139.033 & 644 \\
\hline 99.047 & 10901 & 110.076 & 112525 & 115.476 & 407 & 126.561 & 395 & 139.079 & 1624 \\
\hline 99.082 & 36113 & 110.112 & 103986 & 115.535 & 526 & 126.609 & 562 & 139.114 & 44885 \\
\hline 99.120 & 71071 & 110.169 & 5636 & 116.085 & 11348 & 126.663 & 462 & 139.150 & 36024 \\
\hline
\end{tabular}


Table S32 (continued). High-resolution mass table for the compound identified as $\mathrm{C}_{34} \mathrm{H}_{66} \mathrm{O}_{2}$.

\begin{tabular}{|c|c|c|c|c|c|c|c|c|c|}
\hline Mass & $\begin{array}{c}\text { Ion } \\
\text { counts }\end{array}$ & Mass & $\begin{array}{c}\text { Ion } \\
\text { counts }\end{array}$ & Mass & $\begin{array}{c}\text { Ion } \\
\text { counts }\end{array}$ & Mass & $\begin{array}{c}\text { Ion } \\
\text { counts }\end{array}$ & Mass & $\begin{array}{c}\text { Ion } \\
\text { counts }\end{array}$ \\
\hline 139.216 & 2185 & 154.102 & 3051 & 178.174 & 5136 & 199.172 & 7782 & 227.203 & 7845 \\
\hline 139.342 & 511 & 154.135 & 5953 & 179.146 & 31817 & 200.178 & 1992 & 228.210 & 3315 \\
\hline 139.539 & 296 & 154.174 & 15998 & 179.182 & 6668 & 201.186 & 2015 & 229.218 & 33641 \\
\hline 139.577 & 246 & 154.240 & 611 & 179.259 & 933 & 202.187 & 353 & 229.781 & 413 \\
\hline 139.628 & 488 & 155.110 & 14689 & 180.153 & 12822 & 203.182 & 2576 & 230.222 & 5520 \\
\hline 140.014 & 436 & 155.146 & 14244 & 180.190 & 23164 & 204.190 & 1413 & 230.328 & 536 \\
\hline 140.086 & 7156 & 155.181 & 8403 & 180.297 & 922 & 205.198 & 2699 & 231.221 & 1302 \\
\hline 140.120 & 7556 & 156.116 & 5232 & 180.674 & 414 & 206.205 & 2691 & 232.221 & 577 \\
\hline 140.158 & 23149 & 156.150 & 1727 & 181.126 & 1738 & 207.035 & 1487 & 233.230 & 1158 \\
\hline 140.207 & 387 & 156.186 & 952 & 181.161 & 14008 & 207.177 & 26325 & 234.201 & 1849 \\
\hline 140.242 & 863 & 157.125 & 16250 & 181.196 & 7660 & 207.296 & 738 & 235.208 & 34474 \\
\hline 141.094 & 23741 & 157.223 & 432 & 182.133 & 2045 & 208.038 & 388 & 235.802 & 554 \\
\hline 141.130 & 25081 & 158.132 & 9593 & 182.167 & 2821 & 208.184 & 34598 & 235.889 & 577 \\
\hline 141.166 & 12722 & 159.137 & 2317 & 182.206 & 7924 & 208.220 & 11437 & 236.216 & 275054 \\
\hline 141.222 & 1674 & 160.134 & 446 & 183.140 & 6592 & 208.303 & 1810 & 236.728 & 1782 \\
\hline 141.598 & 478 & 161.136 & 19112 & 183.176 & 2512 & 208.718 & 509 & 236.770 & 1580 \\
\hline 142.101 & 7458 & 161.218 & 541 & 183.212 & 3956 & 209.191 & 13806 & 236.834 & 3277 \\
\hline 142.135 & 2764 & 162.142 & 7156 & 184.147 & 3059 & 209.225 & 5063 & 236.973 & 457 \\
\hline 142.170 & 1645 & 163.114 & 1442 & 184.218 & 600 & 210.195 & 3214 & 237.222 & 105891 \\
\hline 143.109 & 18770 & 163.151 & 13528 & 185.156 & 12427 & 210.235 & 2522 & 237.339 & 6678 \\
\hline 143.147 & 1967 & 163.252 & 466 & 186.164 & 6662 & 211.172 & 8194 & 237.429 & 2144 \\
\hline 143.215 & 514 & 164.123 & 1897 & 187.171 & 2682 & 211.208 & 15097 & 237.831 & 3263 \\
\hline 144.116 & 5635 & 164.158 & 11165 & 188.169 & 365 & 212.178 & 2609 & 238.226 & 17553 \\
\hline 145.113 & 1964 & 165.078 & 671 & 189.167 & 9307 & 212.211 & 2801 & 238.323 & 2050 \\
\hline 146.114 & 640 & 165.130 & 45487 & 190.174 & 5697 & 213.187 & 5004 & 238.369 & 2520 \\
\hline 147.120 & 24946 & 165.167 & 13238 & 191.182 & 9600 & 214.193 & 1586 & 238.824 & 2190 \\
\hline 147.207 & 646 & 165.239 & 1763 & 192.190 & 63024 & 215.203 & 2510 & 239.237 & 8119 \\
\hline 148.127 & 20842 & 165.641 & 447 & 192.702 & 465 & 216.208 & 471 & 239.809 & 1530 \\
\hline 148.218 & 486 & 166.137 & 19422 & 193.162 & 27690 & 217.198 & 29529 & 239.878 & 1321 \\
\hline 149.099 & 2270 & 166.174 & 41247 & 193.196 & 23848 & 218.205 & 30090 & 240.211 & 1197 \\
\hline 149.135 & 24932 & 167.109 & 1585 & 193.275 & 1552 & 218.320 & 1440 & 240.243 & 1673 \\
\hline 149.228 & 559 & 167.146 & 19322 & 193.684 & 549 & 219.213 & 37569 & 240.362 & 660 \\
\hline 150.107 & 4331 & 167.181 & 13478 & 194.169 & 16951 & 219.330 & 1559 & 240.822 & 611 \\
\hline 150.142 & 9132 & 167.254 & 810 & 194.205 & 136964 & 219.707 & 425 & 240.951 & 438 \\
\hline 151.115 & 61913 & 168.117 & 2502 & 194.670 & 862 & 219.857 & 559 & 241.219 & 2498 \\
\hline 151.151 & 25915 & 168.151 & 3743 & 194.709 & 835 & 220.219 & 14264 & 241.374 & 554 \\
\hline 151.221 & 2545 & 168.189 & 7710 & 194.760 & 971 & 220.346 & 461 & 242.227 & 1465 \\
\hline 151.559 & 643 & 168.266 & 414 & 194.811 & 636 & 220.787 & 555 & 243.234 & 8544 \\
\hline 151.650 & 544 & 169.125 & 11490 & 195.142 & 1883 & 221.193 & 8632 & 244.237 & 1707 \\
\hline 151.961 & 568 & 169.162 & 4662 & 195.177 & 9929 & 221.224 & 5412 & 245.228 & 4013 \\
\hline 152.069 & 1279 & 169.197 & 5574 & 195.210 & 27657 & 221.822 & 735 & 246.236 & 3818 \\
\hline 152.122 & 46494 & 170.132 & 4254 & 195.289 & 1773 & 222.200 & 9011 & 247.244 & 3767 \\
\hline 152.159 & 137705 & 170.199 & 798 & 195.632 & 616 & 222.236 & 16894 & 248.248 & 1187 \\
\hline 152.562 & 921 & 171.141 & 16252 & 195.697 & 618 & 223.206 & 4218 & 249.224 & 1567 \\
\hline 152.606 & 1759 & 171.180 & 1157 & 196.148 & 1994 & 223.240 & 4581 & 249.255 & 1262 \\
\hline 153.095 & 3064 & 172.148 & 7476 & 196.181 & 2128 & 223.323 & 526 & 249.943 & 380 \\
\hline 153.130 & 36782 & 173.154 & 3000 & 196.219 & 6046 & 224.179 & 1901 & 250.234 & 4112 \\
\hline 153.165 & 39129 & 174.140 & 458 & 196.659 & 396 & 224.252 & 6454 & 250.267 & 9161 \\
\hline 153.241 & 2171 & 175.151 & 11220 & 197.156 & 5222 & 225.187 & 3587 & 250.371 & 529 \\
\hline 153.389 & 328 & 176.157 & 3936 & 197.227 & 2441 & 225.220 & 2145 & 251.238 & 2810 \\
\hline 153.493 & 535 & 177.166 & 7360 & 198.163 & 2428 & 225.257 & 1770 & 251.270 & 2435 \\
\hline 153.574 & 372 & 178.140 & 796 & 198.233 & 421 & 226.194 & 3895 & 251.946 & 515 \\
\hline
\end{tabular}


Table S32 (continued). High-resolution mass table for the compound identified as $\mathrm{C}_{34} \mathrm{H}_{66} \mathrm{O}_{2}$.

\begin{tabular}{|c|c|c|c|c|c|c|c|c|c|}
\hline Mass & $\begin{array}{c}\text { Ion } \\
\text { counts }\end{array}$ & Mass & $\begin{array}{c}\text { Ion } \\
\text { counts }\end{array}$ & Mass & $\begin{array}{c}\text { Ion } \\
\text { counts }\end{array}$ & Mass & $\begin{array}{c}\text { Ion } \\
\text { counts }\end{array}$ & Mass & $\begin{array}{c}\text { Ion } \\
\text { counts }\end{array}$ \\
\hline 252.211 & 8904 & 265.253 & 21905 & 283.265 & 5846 & 312.302 & 717 & 361.349 & 361 \\
\hline 252.283 & 6018 & 265.380 & 1017 & 284.270 & 1398 & 320.309 & 421 & 365.343 & 1508 \\
\hline 253.218 & 5419 & 266.257 & 4706 & 285.280 & 652 & 321.316 & 491 & 366.346 & 460 \\
\hline 253.289 & 3455 & 267.265 & 1332 & 291.269 & 363 & 323.296 & 1475 & 367.359 & 422 \\
\hline 254.226 & 12062 & 268.243 & 733 & 292.278 & 1523 & 324.301 & 439 & 379.359 & 3302 \\
\hline 254.293 & 1015 & 269.248 & 2784 & 293.284 & 1387 & 325.311 & 2394 & 380.363 & 975 \\
\hline 255.234 & 55176 & 270.256 & 870 & 294.308 & 1497 & 326.315 & 455 & 381.366 & 551 \\
\hline 256.238 & 10744 & 271.264 & 2854 & 295.265 & 2540 & 335.330 & 778 & 393.374 & 691 \\
\hline 256.414 & 775 & 272.267 & 566 & 296.271 & 863 & 336.334 & 372 & 407.393 & 648 \\
\hline 257.249 & 10534 & 275.275 & 293 & 297.281 & 6207 & 337.313 & 582 & 410.414 & 469 \\
\hline 257.390 & 651 & 276.284 & 282 & 298.283 & 1349 & 338.317 & 349 & 424.431 & 636 \\
\hline 257.468 & 524 & 277.285 & 591 & 305.287 & 977 & 339.329 & 421 & 435.415 & 426 \\
\hline 258.251 & 1812 & 278.299 & 11348 & 306.330 & 762 & 347.341 & 374 & 438.448 & 561 \\
\hline 262.234 & 548 & 279.302 & 3986 & 307.301 & 2303 & 349.348 & 672 & 449.431 & 300 \\
\hline 263.240 & 5507 & 280.240 & 1827 & 308.306 & 711 & 350.357 & 592 & 463.453 & 647 \\
\hline 264.247 & 42976 & 280.313 & 874 & 309.280 & 782 & 351.331 & 1110 & 506.505 & 3572 \\
\hline 264.375 & 1822 & 281.248 & 1472 & 310.294 & 386 & 352.330 & 447 & 507.510 & 1850 \\
\hline 264.834 & 429 & 282.257 & 1988 & 311.296 & 778 & 353.343 & 682 & 508.520 & 738 \\
\hline
\end{tabular}


Table S33. High-resolution mass table for the compound identified as $\mathrm{C}_{34} \mathrm{H}_{64} \mathrm{O}_{2}$.

\begin{tabular}{|c|c|c|c|c|c|c|c|}
\hline Mass & $\begin{array}{c}\text { Ion } \\
\text { counts }\end{array}$ & Mass & $\begin{array}{c}\text { Ion } \\
\text { counts }\end{array}$ & Mass & $\begin{array}{c}\text { Ion } \\
\text { counts }\end{array}$ & Mass & $\begin{array}{c}\text { Ion } \\
\text { counts }\end{array}$ \\
\hline 50.018 & 1465 & 58.076 & 23534 & 69.665 & 5616 & 79.057 & 81652 \\
\hline 51.026 & 2887 & 58.117 & 2497 & 69.699 & 5208 & 79.332 & 1031 \\
\hline 52.033 & 1935 & 58.353 & 1969 & 69.948 & 3710 & 79.378 & 779 \\
\hline 53.041 & 26273 & 58.603 & 1457 & 70.044 & 5368 & 79.416 & 658 \\
\hline 53.101 & 675 & 58.647 & 652 & 70.079 & 143162 & 79.467 & 442 \\
\hline 54.011 & 453 & 58.829 & 863 & 70.151 & 4743 & 79.676 & 505 \\
\hline 54.033 & 237 & 58.863 & 855 & 70.197 & 3849 & 79.724 & 458 \\
\hline 54.049 & 182068 & 59.052 & 6042 & 70.241 & 2549 & 80.065 & 87307 \\
\hline 54.088 & 1422 & 59.081 & 1121 & 70.289 & 612 & 80.358 & 548 \\
\hline 54.291 & 1144 & 59.133 & 581 & 70.383 & 434 & 80.389 & 368 \\
\hline 54.315 & 1151 & 59.335 & 462 & 70.446 & 2308 & 80.420 & 418 \\
\hline 54.338 & 1678 & 59.386 & 440 & 70.471 & 1396 & 80.458 & 477 \\
\hline 54.412 & 390 & 60.023 & 15038 & 70.492 & 2053 & 80.652 & 536 \\
\hline 54.566 & 1261 & 60.061 & 318 & 70.541 & 664 & 80.690 & 584 \\
\hline 54.598 & 1316 & 60.087 & 396 & 70.683 & 1006 & 80.966 & 588 \\
\hline 54.757 & 477 & 61.031 & 6413 & 70.728 & 1638 & 81.036 & 3220 \\
\hline 54.788 & 964 & 62.019 & 398 & 70.762 & 1122 & 81.072 & 448463 \\
\hline 54.818 & 1266 & 63.026 & 683 & 70.981 & 1027 & 81.121 & 3577 \\
\hline 54.856 & 1002 & 65.041 & 9819 & 71.013 & 1384 & 81.369 & 2643 \\
\hline 54.915 & 447 & 66.049 & 23591 & 71.052 & 24661 & 81.398 & 2494 \\
\hline 54.977 & 750 & 66.112 & 534 & 71.088 & 258436 & 81.430 & 4267 \\
\hline 55.021 & 21091 & 67.038 & 218 & 71.132 & 2113 & 81.519 & 1053 \\
\hline 55.057 & 660956 & 67.057 & 393527 & 71.197 & 1882 & 81.707 & 3403 \\
\hline 55.096 & 42001 & 67.101 & 3108 & 71.365 & 1259 & 81.756 & 2678 \\
\hline 55.273 & 4288 & 67.326 & 2300 & 71.393 & 1532 & 82.000 & 2684 \\
\hline 55.311 & 3509 & 67.353 & 2239 & 71.421 & 1908 & 82.044 & 6878 \\
\hline 55.325 & 5243 & 67.382 & 3587 & 71.458 & 1576 & 82.080 & 481476 \\
\hline 55.360 & 7616 & 67.463 & 906 & 71.507 & 482 & 82.129 & 3859 \\
\hline 55.401 & 3670 & 67.626 & 2733 & 71.660 & 1243 & 82.378 & 3126 \\
\hline 55.423 & 972 & 67.653 & 1773 & 71.700 & 1726 & 82.408 & 2469 \\
\hline 55.432 & 515 & 67.678 & 2612 & 71.924 & 680 & 82.439 & 3691 \\
\hline 55.600 & 6201 & 67.925 & 2314 & 71.955 & 520 & 82.530 & 939 \\
\hline 55.843 & 4597 & 67.992 & 704 & 71.984 & 639 & 82.722 & 3101 \\
\hline 56.027 & 4794 & 68.028 & 1646 & 72.020 & 400 & 82.994 & 2739 \\
\hline 56.064 & 170295 & 68.064 & 251585 & 72.057 & 2035 & 83.052 & 36015 \\
\hline 56.311 & 3421 & 68.337 & 912 & 72.092 & 13734 & 83.088 & 623277 \\
\hline 56.334 & 3640 & 68.362 & 1437 & 72.166 & 427 & 83.355 & 3049 \\
\hline 56.369 & 2673 & 68.391 & 2318 & 72.211 & 636 & 83.395 & 4187 \\
\hline 56.570 & 2634 & 68.426 & 2663 & 72.254 & 380 & 83.418 & 4194 \\
\hline 56.827 & 2127 & 68.451 & 861 & 72.284 & 449 & 83.455 & 6759 \\
\hline 57.036 & 16033 & 68.472 & 1206 & 72.500 & 534 & 83.517 & 1486 \\
\hline 57.073 & 504961 & 68.658 & 2328 & 73.031 & 29350 & 83.535 & 994 \\
\hline 57.136 & 12542 & 68.907 & 1583 & 73.067 & 6859 & 83.546 & 1015 \\
\hline 57.278 & 681 & 68.945 & 2055 & 73.343 & 257 & 83.749 & 4992 \\
\hline 57.320 & 3618 & 68.982 & 1805 & 73.370 & 446 & 84.013 & 3571 \\
\hline 57.346 & 3196 & 69.036 & 6213 & 73.652 & 418 & 84.060 & 150929 \\
\hline 57.370 & 5001 & 69.073 & 646127 & 74.038 & 3356 & 84.094 & 91670 \\
\hline 57.427 & 743 & 69.373 & 4309 & 74.074 & 319 & 84.137 & 5295 \\
\hline 57.446 & 906 & 69.410 & 6722 & 75.030 & 1010 & 84.231 & 1245 \\
\hline 57.609 & 4177 & 69.464 & 3803 & 76.035 & 438 & 84.358 & 2757 \\
\hline 57.852 & 3009 & 69.482 & 940 & 77.041 & 11369 & 84.391 & 1919 \\
\hline 58.043 & 3819 & 69.492 & 1173 & 78.049 & 9222 & 84.419 & 2464 \\
\hline
\end{tabular}


Table S33 (continued). High-resolution mass table for the compound identified as $\mathrm{C}_{34} \mathrm{H}_{64} \mathrm{O}_{2}$.

\begin{tabular}{|c|c|c|c|c|c|c|c|}
\hline Mass & $\begin{array}{c}\text { Ion } \\
\text { counts }\end{array}$ & Mass & $\begin{array}{c}\text { Ion } \\
\text { counts }\end{array}$ & Mass & $\begin{array}{c}\text { Ion } \\
\text { counts }\end{array}$ & Mass & $\begin{array}{c}\text { Ion } \\
\text { counts }\end{array}$ \\
\hline 84.593 & 444 & 95.779 & 2997 & 103.076 & 1733 & \begin{tabular}{|l|}
113.062 \\
\end{tabular} & 16786 \\
\hline 84.649 & 1654 & 96.059 & 58156 & 104.064 & 1405 & \begin{tabular}{|l|}
113.098 \\
\end{tabular} & 7710 \\
\hline 84.687 & 1940 & 96.096 & 429199 & 105.073 & 9576 & 113.135 & 16601 \\
\hline 84.962 & 1069 & 96.131 & 1465 & 106.080 & 5626 & \begin{tabular}{|l|}
113.220 \\
\end{tabular} & 1863 \\
\hline 85.031 & 4732 & 96.146 & 4348 & 107.051 & 1254 & 113.541 & 703 \\
\hline 85.066 & 30933 & 96.420 & 4390 & 107.088 & 36941 & \begin{tabular}{|l|l|}
113.591 \\
\end{tabular} & 700 \\
\hline 85.104 & 149462 & 96.450 & 3245 & 107.169 & 1104 & 113.633 & 510 \\
\hline 85.151 & 1690 & 96.483 & 5281 & 107.494 & 427 & 113.868 & 470 \\
\hline 85.402 & 697 & 96.580 & 498 & 108.060 & 1135 & 113.911 & 388 \\
\hline 85.438 & 1255 & 96.745 & 3357 & 108.095 & 33433 & \begin{tabular}{|l|}
113.951 \\
\end{tabular} & 503 \\
\hline 85.489 & 1283 & 96.780 & 4155 & 108.507 & 424 & \begin{tabular}{|l|}
114.070 \\
\end{tabular} & 25406 \\
\hline 85.529 & 728 & 96.820 & 3850 & 108.801 & 410 & \begin{tabular}{|l|}
114.107 \\
\end{tabular} & 490 \\
\hline 85.565 & 445 & 97.035 & 3195 & 108.853 & 449 & \begin{tabular}{|l|}
114.139 \\
\end{tabular} & 2245 \\
\hline 85.744 & 607 & 97.067 & 82354 & 109.068 & 16574 & \begin{tabular}{|l|}
114.222 \\
\end{tabular} & 548 \\
\hline 85.788 & 540 & 97.104 & 515670 & 109.104 & 226132 & 114.268 & 423 \\
\hline 85.821 & 561 & 97.184 & 15867 & 109.159 & 2152 & \begin{tabular}{|l|}
115.078 \\
\end{tabular} & 33926 \\
\hline 86.039 & 2619 & 97.428 & 5743 & 109.448 & 1529 & 115.115 & 2418 \\
\hline 86.071 & 2796 & 97.460 & 4278 & 109.481 & 1466 & 116.084 & 5523 \\
\hline 86.107 & 9455 & 97.479 & 2927 & 109.517 & 2379 & 117.073 & 2952 \\
\hline 86.360 & 427 & 97.509 & 5981 & 109.593 & 502 & 118.081 & 1765 \\
\hline 86.401 & 467 & 97.563 & 1143 & 109.623 & 307 & \begin{tabular}{|l|}
119.088 \\
\end{tabular} & 18571 \\
\hline 86.439 & 280 & 97.590 & 499 & 109.837 & 2052 & 120.095 & 6258 \\
\hline 87.047 & 25396 & 97.803 & 5333 & 109.922 & 949 & \begin{tabular}{|l|}
121.067 \\
\end{tabular} & 4247 \\
\hline 87.083 & 2961 & 98.075 & 97933 & 110.075 & 45784 & \begin{tabular}{|l|}
121.104 \\
\end{tabular} & 65494 \\
\hline 87.111 & 947 & 98.109 & 64154 & 110.111 & 173043 & \begin{tabular}{|l|}
121.474 \\
\end{tabular} & 276 \\
\hline 88.052 & 2623 & 98.159 & 6485 & 110.460 & 1769 & 121.503 & 324 \\
\hline 88.089 & 323 & 98.434 & 4002 & 110.494 & 1768 & \begin{tabular}{|l|}
121.535 \\
\end{tabular} & 364 \\
\hline 89.063 & 1252 & 98.476 & 3720 & 110.530 & 2934 & 121.875 & 477 \\
\hline 91.057 & 18235 & 98.750 & 2915 & 110.588 & 1440 & \begin{tabular}{|l|}
122.075 \\
\end{tabular} & 3838 \\
\hline 91.125 & 458 & 99.047 & 7786 & 110.840 & 2128 & \begin{tabular}{|l|}
122.111 \\
\end{tabular} & 28875 \\
\hline 92.064 & 6675 & 99.082 & 20653 & 110.881 & 2396 & \begin{tabular}{|l|}
122.194 \\
\end{tabular} & 1175 \\
\hline 93.072 & 55610 & 99.119 & 31386 & 111.047 & 940 & 122.520 & 401 \\
\hline 93.389 & 411 & 99.166 & 1938 & 111.083 & 59302 & 122.583 & 512 \\
\hline 93.429 & 519 & 99.380 & 1358 & 111.119 & 209843 & 122.916 & 249 \\
\hline 94.044 & 1328 & 99.438 & 1932 & 111.174 & 4009 & 122.949 & 364 \\
\hline 94.080 & 61552 & 99.728 & 1082 & 111.204 & 8283 & 122.995 & 486 \\
\hline 94.391 & 394 & 99.760 & 1315 & 111.467 & 2024 & 123.083 & 51628 \\
\hline 94.420 & 521 & 99.831 & 460 & 111.501 & 1849 & \begin{tabular}{|l|}
123.119 \\
\end{tabular} & 129166 \\
\hline 94.462 & 534 & 100.054 & 12496 & 111.538 & 3482 & \begin{tabular}{|l|}
123.482 \\
\end{tabular} & 992 \\
\hline 94.523 & 381 & 100.087 & 1932 & 111.640 & 408 & 123.523 & 977 \\
\hline 94.719 & 398 & 100.123 & 3183 & 111.882 & 2837 & \begin{tabular}{|l|}
123.561 \\
\end{tabular} & 1779 \\
\hline 94.759 & 484 & 100.378 & 523 & 112.054 & 1716 & \begin{tabular}{|l|}
123.616 \\
\end{tabular} & 1050 \\
\hline 94.950 & 429 & 100.420 & 612 & 112.090 & 18801 & 123.666 & 340 \\
\hline 94.987 & 339 & 100.456 & 546 & 112.125 & 33923 & 123.833 & 488 \\
\hline 95.052 & 9066 & 100.703 & 456 & 112.194 & 3416 & \begin{tabular}{|l|}
123.916 \\
\end{tabular} & 1461 \\
\hline 95.088 & 403419 & 100.751 & 465 & 112.552 & 1913 & 124.025 & 414 \\
\hline 95.408 & 2489 & 101.010 & 435 & 112.601 & 1665 & \begin{tabular}{|l|}
124.090 \\
\end{tabular} & 22350 \\
\hline 95.441 & 2331 & 101.062 & 37240 & 112.825 & 422 & \begin{tabular}{|l|}
124.127 \\
\end{tabular} & 114783 \\
\hline 95.471 & 3082 & 101.099 & 2827 & 112.863 & 736 & 124.185 & 1738 \\
\hline 95.505 & 3050 & 101.415 & 496 & 112.889 & 966 & \begin{tabular}{|l|}
124.497 \\
\end{tabular} & 505 \\
\hline 95.545 & 715 & 102.068 & 4481 & 112.919 & 1257 & \begin{tabular}{|l|l|}
124.530 \\
\end{tabular} & 752 \\
\hline 95.572 & 356 & 102.143 & 372 & 112.961 & 616 & 124.577 & 1620 \\
\hline
\end{tabular}


Table S33 (continued). High-resolution mass table for the compound identified as $\mathrm{C}_{34} \mathrm{H}_{64} \mathrm{O}_{2}$.

\begin{tabular}{|c|c|c|c|c|c|c|c|}
\hline Mass & $\begin{array}{c}\text { Ion } \\
\text { counts }\end{array}$ & Mass & $\begin{array}{c}\text { Ion } \\
\text { counts }\end{array}$ & Mass & $\begin{array}{c}\text { Ion } \\
\text { counts }\end{array}$ & Mass & $\begin{array}{c}\text { Ion } \\
\text { counts }\end{array}$ \\
\hline 124.677 & 473 & 138.105 & 19557 & 153.129 & 30139 & \begin{tabular}{|l|}
178.137 \\
\end{tabular} & 2613 \\
\hline 124.911 & 1095 & 138.142 & 83320 & 153.164 & 23369 & \begin{tabular}{|l|l|}
178.173 \\
\end{tabular} & 11040 \\
\hline 124.973 & 867 & 138.207 & 1424 & 153.238 & 1485 & 179.145 & 13512 \\
\hline 125.061 & 1451 & 138.567 & 711 & 154.101 & 4845 & \begin{tabular}{|l|}
179.181 \\
\end{tabular} & 16408 \\
\hline 125.098 & 40930 & 138.642 & 555 & 154.134 & 4197 & \begin{tabular}{|l|}
179.272 \\
\end{tabular} & 740 \\
\hline 125.134 & 79829 & 138.693 & 448 & 154.172 & 7658 & 180.151 & 5307 \\
\hline 125.538 & 699 & 138.979 & 486 & 155.109 & 8120 & \begin{tabular}{|l|}
180.189 \\
\end{tabular} & 28406 \\
\hline 125.605 & 1240 & 139.078 & 2698 & 155.145 & 6263 & \begin{tabular}{|l|l|}
180.253 \\
\end{tabular} & 592 \\
\hline 125.688 & 430 & 139.114 & 37084 & 155.181 & 3184 & \begin{tabular}{|l|}
180.299 \\
\end{tabular} & 780 \\
\hline 125.942 & 587 & 139.149 & 30819 & 156.115 & 2363 & \begin{tabular}{|l|}
181.124 \\
\end{tabular} & 4510 \\
\hline 125.987 & 680 & 139.610 & 391 & 156.150 & 1011 & \begin{tabular}{|l|}
181.161 \\
\end{tabular} & 14687 \\
\hline 126.070 & 10244 & 140.085 & 8918 & 157.124 & 14044 & \begin{tabular}{|l|}
181.194 \\
\end{tabular} & 8197 \\
\hline 126.104 & 6531 & 140.119 & 4555 & 157.163 & 1037 & 182.132 & 2964 \\
\hline 126.141 & 18585 & 140.157 & 10510 & 158.131 & 3446 & 182.165 & 2706 \\
\hline 126.234 & 793 & 140.232 & 448 & 159.119 & 2069 & \begin{tabular}{|l|}
182.204 \\
\end{tabular} & 3557 \\
\hline 127.078 & 30214 & 141.093 & 13503 & 160.122 & 507 & \begin{tabular}{|l|}
183.139 \\
\end{tabular} & 3359 \\
\hline 127.114 & 5617 & 141.130 & 10079 & 161.135 & 11717 & 183.175 & 1797 \\
\hline 127.150 & 9610 & 141.166 & 5651 & 162.140 & 3668 & 183.211 & 1293 \\
\hline 127.225 & 600 & 141.272 & 474 & 163.114 & 3798 & 184.147 & 1408 \\
\hline 127.506 & 453 & 142.099 & 3674 & 163.150 & 19836 & \begin{tabular}{|l|}
185.155 \\
\end{tabular} & 11214 \\
\hline 128.084 & 8743 & 142.133 & 1141 & 163.248 & 678 & \begin{tabular}{|l|}
185.272 \\
\end{tabular} & 339 \\
\hline 128.119 & 448 & 142.170 & 619 & 164.121 & 5419 & 186.161 & 2893 \\
\hline 128.154 & 1041 & 143.109 & 16950 & 164.157 & 13540 & \begin{tabular}{|l|}
187.158 \\
\end{tabular} & 1679 \\
\hline 129.093 & 25414 & 143.146 & 1150 & 165.073 & 555 & \begin{tabular}{|l|}
188.155 \\
\end{tabular} & 409 \\
\hline 129.132 & 1506 & 143.202 & 545 & 165.129 & 20449 & 189.166 & 5676 \\
\hline 129.189 & 537 & 144.114 & 2794 & 165.166 & 24228 & \begin{tabular}{|l|}
190.172 \\
\end{tabular} & 3018 \\
\hline 130.098 & 3411 & 145.105 & 2300 & 165.240 & 1133 & \begin{tabular}{|l|}
191.004 \\
\end{tabular} & 360 \\
\hline 131.088 & 3572 & 146.112 & 1021 & 166.136 & 8258 & \begin{tabular}{|l|}
191.146 \\
\end{tabular} & 1474 \\
\hline 132.094 & 1486 & 147.086 & 842 & 166.173 & 41248 & \begin{tabular}{|l|}
191.181 \\
\end{tabular} & 14177 \\
\hline 133.020 & 410 & 147.119 & 15230 & 166.274 & 1336 & \begin{tabular}{|l|}
191.289 \\
\end{tabular} & 469 \\
\hline 133.103 & 20758 & 147.208 & 463 & 167.108 & 4310 & 192.153 & 1432 \\
\hline 133.203 & 571 & 148.126 & 8197 & 167.145 & 18948 & \begin{tabular}{|l|}
192.189 \\
\end{tabular} & 36565 \\
\hline 134.111 & 11623 & 149.099 & 6046 & 167.179 & 11694 & \begin{tabular}{|l|}
192.297 \\
\end{tabular} & 1159 \\
\hline 135.083 & 7015 & 149.135 & 33368 & 167.263 & 963 & \begin{tabular}{|l|}
193.161 \\
\end{tabular} & 11465 \\
\hline 135.119 & 57069 & 149.230 & 816 & 168.116 & 3566 & 193.196 & 19967 \\
\hline 135.540 & 439 & 150.106 & 12187 & 168.150 & 2853 & 193.277 & 820 \\
\hline 135.599 & 471 & 150.142 & 16885 & 168.188 & 4464 & \begin{tabular}{|l|}
193.682 \\
\end{tabular} & 425 \\
\hline 135.950 & 513 & 150.211 & 394 & 169.124 & 5443 & \begin{tabular}{|l|l|}
194.168 \\
\end{tabular} & 5965 \\
\hline 136.091 & 9760 & 150.236 & 492 & 169.161 & 5864 & \begin{tabular}{|l|}
194.204 \\
\end{tabular} & 48001 \\
\hline 136.126 & 23188 & 151.114 & 30347 & 169.196 & 2190 & \begin{tabular}{|l|}
194.302 \\
\end{tabular} & 1950 \\
\hline 136.217 & 917 & 151.150 & 39922 & 170.130 & 1933 & \begin{tabular}{|l|}
194.734 \\
\end{tabular} & 513 \\
\hline 136.272 & 576 & 151.234 & 2081 & 170.164 & 1012 & \begin{tabular}{|l|}
195.140 \\
\end{tabular} & 4177 \\
\hline 137.047 & 790 & 151.610 & 430 & 171.140 & 13224 & \begin{tabular}{|l|l|}
195.176 \\
\end{tabular} & 9306 \\
\hline 137.098 & 36037 & 151.654 & 471 & 171.177 & 1492 & \begin{tabular}{|l|}
195.209 \\
\end{tabular} & 12190 \\
\hline 137.135 & 76620 & 151.988 & 467 & 172.145 & 2825 & \begin{tabular}{|l|}
195.315 \\
\end{tabular} & 608 \\
\hline 137.228 & 2797 & 152.067 & 1078 & 173.136 & 2282 & \begin{tabular}{|c|}
195.647 \\
\end{tabular} & 466 \\
\hline 137.525 & 448 & 152.121 & 15695 & 174.142 & 546 & \begin{tabular}{|l|}
196.147 \\
\end{tabular} & 2376 \\
\hline 137.563 & 497 & 152.158 & 80200 & 175.150 & 7124 & \begin{tabular}{|l|}
196.181 \\
\end{tabular} & 1997 \\
\hline 137.612 & 708 & 152.253 & 2942 & 176.157 & 2261 & \begin{tabular}{|l|}
196.217 \\
\end{tabular} & 3131 \\
\hline 137.898 & 443 & 152.607 & 621 & 177.130 & 2509 & \begin{tabular}{|l|}
197.155 \\
\end{tabular} & 2336 \\
\hline 137.942 & 639 & 152.677 & 542 & 177.166 & 11226 & \begin{tabular}{|l|}
197.188 \\
\end{tabular} & 1201 \\
\hline 137.991 & 433 & 153.093 & 3948 & 177.243 & 433 & 197.226 & 1092 \\
\hline
\end{tabular}


Table S33 (continued). High-resolution mass table for the compound identified as $\mathrm{C}_{34} \mathrm{H}_{64} \mathrm{O}_{2}$.

\begin{tabular}{|c|c|c|c|c|c|c|c|}
\hline Mass & $\begin{array}{c}\text { Ion } \\
\text { counts }\end{array}$ & Mass & $\begin{array}{c}\text { Ion } \\
\text { counts }\end{array}$ & Mass & $\begin{array}{c}\text { Ion } \\
\text { counts }\end{array}$ & Mass & $\begin{array}{c}\text { Ion } \\
\text { counts }\end{array}$ \\
\hline 198.162 & 1155 & 223.171 & 2849 & 249.934 & 471 & 283.264 & 10420 \\
\hline 199.171 & 8200 & 223.207 & 4585 & 250.231 & 3128 & 284.267 & 2646 \\
\hline 200.176 & 1701 & 223.239 & 4520 & 250.266 & 7365 & 285.279 & 4711 \\
\hline 201.178 & 1081 & 223.371 & 526 & 251.202 & 12257 & 286.281 & 888 \\
\hline 202.178 & 306 & 224.179 & 2546 & 252.209 & 10985 & 287.277 & 353 \\
\hline 203.182 & 2121 & 224.210 & 1315 & 252.352 & 467 & 289.253 & 746 \\
\hline 204.191 & 1024 & 224.249 & 1824 & 253.013 & 296 & 289.287 & 527 \\
\hline 205.161 & 1170 & 225.185 & 2203 & 253.215 & 4144 & 290.262 & 2778 \\
\hline 205.197 & 4900 & 225.221 & 2029 & 254.224 & 3255 & 291.270 & 1749 \\
\hline 206.168 & 2736 & 226.193 & 1417 & 255.020 & 346 & 291.303 & 1361 \\
\hline 206.204 & 8913 & 227.202 & 10036 & 255.233 & 54418 & 292.276 & 1680 \\
\hline 207.034 & 4224 & 227.323 & 461 & 255.364 & 2176 & 292.310 & 1479 \\
\hline 207.176 & 10760 & 228.206 & 2118 & 255.817 & 588 & 293.249 & 1858 \\
\hline 207.211 & 9077 & 229.217 & 5169 & 256.237 & 10890 & 293.283 & 4161 \\
\hline 207.331 & 538 & 230.219 & 995 & 257.248 & 19552 & 294.255 & 1947 \\
\hline 208.035 & 942 & 231.214 & 1006 & 258.252 & 3579 & 294.284 & 1920 \\
\hline 208.183 & 11009 & 232.219 & 476 & 259.249 & 794 & 295.272 & 1930 \\
\hline 208.220 & 13831 & 233.191 & 1385 & 261.222 & 1564 & 296.283 & 494 \\
\hline 208.300 & 757 & 233.227 & 3172 & 261.258 & 1823 & 297.281 & 484 \\
\hline 209.036 & 420 & 234.199 & 20869 & 262.230 & 12934 & 299.266 & 406 \\
\hline 209.157 & 2818 & 234.946 & 353 & 263.237 & 8803 & 303.303 & 1563 \\
\hline 209.191 & 16373 & 235.206 & 18355 & 263.271 & 3387 & 304.312 & 8899 \\
\hline 209.224 & 5182 & 235.241 & 5441 & 263.388 & 502 & 305.317 & 4439 \\
\hline 210.163 & 1883 & 235.341 & 681 & 264.245 & 28951 & 306.328 & 14258 \\
\hline 210.196 & 3231 & 235.413 & 387 & 264.373 & 1208 & 306.455 & 607 \\
\hline 210.233 & 1946 & 236.214 & 62738 & 265.253 & 40061 & 307.263 & 3665 \\
\hline 211.171 & 2849 & 236.324 & 3119 & 265.381 & 1929 & 307.330 & 3995 \\
\hline 211.207 & 2841 & 236.772 & 637 & 265.841 & 496 & 308.270 & 3441 \\
\hline 212.177 & 1278 & 236.832 & 847 & 265.948 & 527 & 308.335 & 1046 \\
\hline 213.186 & 5391 & 237.222 & 101119 & 266.256 & 9118 & 309.278 & 1425 \\
\hline 214.191 & 1248 & 237.792 & 1484 & 266.361 & 428 & 310.286 & 640 \\
\hline 215.189 & 1506 & 238.226 & 19242 & 266.493 & 444 & 311.292 & 798 \\
\hline 216.189 & 864 & 238.332 & 1357 & 267.231 & 895 & 313.293 & 426 \\
\hline 217.196 & 12684 & 238.388 & 1423 & 267.267 & 3168 & 315.031 & 356 \\
\hline 217.294 & 476 & 238.784 & 877 & 268.260 & 953 & 317.300 & 451 \\
\hline 218.203 & 8438 & 238.847 & 1019 & 269.248 & 1383 & 318.290 & 1069 \\
\hline 219.212 & 50809 & 239.237 & 11118 & 270.255 & 554 & 319.315 & 1575 \\
\hline 219.326 & 1950 & 239.367 & 912 & 271.263 & 1964 & 320.319 & 2151 \\
\hline 219.753 & 339 & 239.798 & 1213 & 272.264 & 461 & 321.314 & 9044 \\
\hline 219.815 & 615 & 239.882 & 893 & 273.261 & 330 & 322.317 & 4828 \\
\hline 220.218 & 24457 & 240.242 & 2399 & 275.274 & 2352 & 323.306 & 2271 \\
\hline 220.326 & 1277 & 241.216 & 2599 & 276.282 & 7599 & 324.312 & 546 \\
\hline 220.744 & 520 & 242.222 & 818 & 277.288 & 5117 & 325.313 & 513 \\
\hline 220.830 & 506 & 243.232 & 5068 & 278.297 & 14278 & 331.310 & 446 \\
\hline 221.192 & 5812 & 244.229 & 1411 & 278.424 & 609 & 332.338 & 1456 \\
\hline 221.226 & 10757 & 245.228 & 5083 & 279.233 & 10067 & 333.336 & 1589 \\
\hline 221.313 & 572 & 246.233 & 3008 & 279.300 & 3590 & 334.358 & 1977 \\
\hline 221.765 & 866 & 247.243 & 11852 & 280.240 & 7252 & 335.294 & 3088 \\
\hline 221.819 & 968 & 247.371 & 598 & 280.310 & 1228 & 336.300 & 2418 \\
\hline 222.199 & 4037 & 248.249 & 5435 & 281.056 & 602 & 337.310 & 1168 \\
\hline 222.235 & 18610 & 249.223 & 2113 & 281.246 & 2741 & 339.330 & 435 \\
\hline 222.766 & 507 & 249.258 & 5312 & 282.255 & 1384 & 341.265 & 418 \\
\hline
\end{tabular}


Table S33 (continued). High-resolution mass table for the compound identified as $\mathrm{C}_{34} \mathrm{H}_{64} \mathrm{O}_{2}$.

\begin{tabular}{|c|c|c|c|c|c|c|c|}
\hline Mass & $\begin{array}{c}\text { Ion } \\
\text { counts }\end{array}$ & Mass & $\begin{array}{c}\text { Ion } \\
\text { counts }\end{array}$ & Mass & $\begin{array}{c}\text { Ion } \\
\text { counts }\end{array}$ & Mass & $\begin{array}{c}\text { Ion } \\
\text { counts }\end{array}$ \\
\hline 345.330 & 442 & 377.047 & 487 & 419.387 & 3723 & 475.448 & 2444 \\
\hline 346.327 & 582 & 377.341 & 2240 & 420.393 & 4071 & 476.456 & 2196 \\
\hline 347.348 & 824 & 378.345 & 2248 & 421.400 & 1644 & 477.461 & 850 \\
\hline 348.335 & 772 & 379.354 & 1269 & 429.407 & 782 & 487.472 & 503 \\
\hline 349.309 & 1422 & 387.369 & 682 & 430.416 & 605 & 489.466 & 1999 \\
\hline 349.338 & 1860 & 388.367 & 760 & 431.417 & 676 & 490.471 & 1552 \\
\hline 350.319 & 2206 & 389.372 & 916 & 432.424 & 566 & 491.482 & 515 \\
\hline 351.321 & 1421 & 390.377 & 578 & 433.403 & 3950 & 501.469 & 391 \\
\hline 352.335 & 392 & 391.357 & 2571 & 434.409 & 4020 & 503.486 & 1441 \\
\hline 353.343 & 411 & 392.362 & 2728 & 435.415 & 1476 & 504.485 & 1109 \\
\hline 359.356 & 355 & 393.372 & 1009 & 439.064 & 668 & 505.489 & 348 \\
\hline 360.340 & 545 & 401.377 & 873 & 443.425 & 410 & 517.495 & 1185 \\
\hline 361.346 & 964 & 402.385 & 1059 & 444.432 & 345 & 518.500 & 802 \\
\hline 362.354 & 530 & 403.387 & 848 & 445.429 & 529 & 531.503 & 858 \\
\hline 363.325 & 2592 & 404.390 & 487 & 447.418 & 3350 & 532.520 & 523 \\
\hline 364.331 & 2324 & 405.371 & 3246 & 448.424 & 3103 & 558.531 & 772 \\
\hline 365.339 & 839 & 406.379 & 3438 & 449.429 & 1293 & 559.531 & 504 \\
\hline 366.338 & 318 & 407.383 & 1513 & 461.434 & 2838 & 560.549 & 11904 \\
\hline 373.363 & 486 & 415.395 & 918 & 462.440 & 3033 & 561.554 & 5385 \\
\hline 374.353 & 447 & 416.402 & 598 & 463.444 & 1179 & 562.555 & 1322 \\
\hline 375.366 & 986 & 417.412 & 660 & 473.439 & 496 & 564.578 & 422 \\
\hline
\end{tabular}


Table S34. High-resolution mass table for the compound identified as cholesteryl myristate.

\begin{tabular}{|c|c|c|c|c|c|c|c|c|c|}
\hline Mass & $\begin{array}{c}\text { Ion } \\
\text { counts }\end{array}$ & Mass & $\begin{array}{c}\text { Ion } \\
\text { counts }\end{array}$ & Mass & $\begin{array}{c}\text { Ion } \\
\text { counts }\end{array}$ & Mass & $\begin{array}{c}\text { Ion } \\
\text { counts }\end{array}$ & Mass & $\begin{array}{c}\text { Ion } \\
\text { counts }\end{array}$ \\
\hline 53.038 & 51 & 95.086 & 2178 & 132.092 & 303 & 177.165 & 168 & 246.235 & 85 \\
\hline 54.046 & 53 & 96.004 & 64 & 133.014 & 9 & 185.133 & 474 & 247.243 & 1578 \\
\hline 55.018 & 79 & 96.092 & 333 & 133.101 & 1510 & 186.142 & 80 & 248.246 & 190 \\
\hline 55.054 & 2649 & 96.505 & 124 & 134.108 & 581 & 187.149 & 313 & 248.992 & 5 \\
\hline 56.062 & 82 & 97.065 & 77 & 135.117 & 1486 & 189.164 & 108 & 255.211 & 4201 \\
\hline 57.033 & 221 & 97.101 & 1170 & 136.123 & 274 & 191.002 & 160 & 256.215 & 946 \\
\hline 57.070 & 3230 & 98.073 & 103 & 137.132 & 271 & 191.180 & 271 & 257.222 & 137 \\
\hline 58.073 & 22 & 99.072 & 42 & 141.072 & 65 & 193.197 & 71 & 259.242 & 297 \\
\hline 60.021 & 257 & 99.118 & 81 & 142.077 & 45 & 197.074 & 67 & 260.250 & 1257 \\
\hline 61.030 & 53 & 104.060 & 68 & 143.086 & 1010 & 197.131 & 84 & 261.255 & 346 \\
\hline 67.054 & 552 & 105.070 & 2038 & 144.092 & 250 & 199.149 & 558 & 265.019 & 142 \\
\hline 68.063 & 302 & 106.077 & 680 & 145.101 & 2574 & 200.153 & 71 & 274.264 & 48 \\
\hline 69.070 & 972 & 107.086 & 2113 & 146.108 & 899 & 201.164 & 486 & 281.052 & 138 \\
\hline 70.078 & 93 & 108.093 & 784 & 147.117 & 3803 & 205.195 & 124 & 282.052 & 218 \\
\hline 71.086 & 1421 & 109.101 & 1349 & 148.123 & 939 & 206.202 & 18 & 283.049 & 20 \\
\hline 73.029 & 330 & 110.106 & 128 & 149.133 & 1023 & 207.033 & 1740 & 301.283 & 51 \\
\hline 73.047 & 54 & 111.079 & 1 & 151.147 & 72 & 207.209 & 2876 & 326.296 & 141 \\
\hline 77.039 & 161 & 111.117 & 619 & 155.085 & 48 & 208.033 & 31 & 353.321 & 2871 \\
\hline 78.046 & 39 & 115.053 & 15 & 157.102 & 576 & 209.031 & 52 & 354.325 & 895 \\
\hline 79.054 & 848 & 117.070 & 653 & 158.108 & 429 & 210.031 & 50 & 355.332 & 54 \\
\hline 80.061 & 13 & 118.078 & 292 & 159.117 & 1403 & 211.208 & 18 & 367.344 & 75 \\
\hline 81.070 & 2325 & 119.086 & 1527 & 160.124 & 886 & 212.947 & 48 & 368.344 & 3341 \\
\hline 81.131 & 1048 & 120.093 & 1255 & 161.133 & 1706 & 213.164 & 3062 & 369.350 & 1725 \\
\hline 82.078 & 321 & 121.101 & 1859 & 162.137 & 251 & 214.168 & 644 & 370.354 & 250 \\
\hline 83.048 & 64 & 122.108 & 523 & 163.148 & 621 & 215.179 & 428 & 377.048 & 31 \\
\hline 83.086 & 1909 & 123.117 & 924 & 165.165 & 27 & 216.186 & 58 & 439.063 & 29 \\
\hline 84.058 & 52 & 124.123 & 68 & 171.117 & 417 & 219.214 & 77 & 581.530 & 64 \\
\hline 84.090 & 95 & 125.133 & 82 & 172.124 & 59 & 227.179 & 202 & 582.532 & 95 \\
\hline 85.101 & 389 & 128.062 & 30 & 173.133 & 769 & 228.187 & 546 & 596.554 & 845 \\
\hline 91.054 & 1374 & 129.070 & 212 & 174.140 & 104 & 229.196 & 1133 & 597.557 & 273 \\
\hline 92.060 & 169 & 129.091 & 83 & 175.148 & 380 & 230.200 & 209 & & \\
\hline 93.070 & 1541 & 130.077 & 79 & 176.153 & 138 & 241.195 & 132 & & \\
\hline 94.077 & 385 & 131.086 & 1266 & 176.987 & 142 & 245.226 & 279 & & \\
\hline
\end{tabular}


Table S35. High-resolution mass table for the compound identified as cholesteryl palmitate.

\begin{tabular}{|c|c|c|c|c|c|c|c|c|c|}
\hline Mass & $\begin{array}{c}\text { Ion } \\
\text { counts }\end{array}$ & Mass & $\begin{array}{c}\text { Ion } \\
\text { counts }\end{array}$ & Mass & $\begin{array}{c}\text { Ion } \\
\text { counts }\end{array}$ & Mass & $\begin{array}{c}\text { Ion } \\
\text { counts }\end{array}$ & Mass & $\begin{array}{c}\text { Ion } \\
\text { counts }\end{array}$ \\
\hline 53.039 & 46 & 107.086 & 4309 & 149.133 & 2615 & 205.196 & 638 & 269.227 & 162 \\
\hline 54.046 & 64 & 108.093 & 1222 & \begin{tabular}{|l|l|}
150.137 \\
\end{tabular} & 354 & 206.201 & 376 & 273.258 & 308 \\
\hline 55.018 & 30 & 109.063 & 369 & 151.149 & 415 & 207.033 & 4885 & 274.266 & 327 \\
\hline 55.054 & 3454 & 109.102 & 3278 & 152.155 & 161 & 207.212 & 50 & 275.271 & 113 \\
\hline 56.062 & 118 & 110.105 & 268 & \begin{tabular}{|l|}
153.072 \\
\end{tabular} & 61 & 208.034 & 126 & 281.053 & 779 \\
\hline 57.070 & 5062 & 111.081 & 136 & 155.084 & 30 & 209.030 & 225 & 282.052 & 122 \\
\hline 58.074 & 201 & 111.117 & 1009 & 156.092 & 94 & 211.149 & 98 & 283.049 & 79 \\
\hline 60.021 & 121 & 112.119 & 42 & \begin{tabular}{|l|l|}
157.102 \\
\end{tabular} & 1439 & 212.156 & 71 & 283.243 & 225 \\
\hline 67.055 & 600 & 113.134 & 30 & 158.109 & 836 & 213.165 & 7829 & 285.261 & 113 \\
\hline 68.062 & 3 & 115.055 & 137 & 159.117 & 3048 & 213.226 & 87 & 287.273 & 334 \\
\hline 69.070 & 2816 & 117.070 & 1226 & 160.125 & 1657 & 214.169 & 1732 & 288.280 & 56 \\
\hline 70.078 & 260 & 118.077 & 566 & 161.133 & 4207 & 215.180 & 1556 & 299.270 & 81 \\
\hline 71.050 & 269 & 119.086 & 3729 & 162.138 & 865 & 216.185 & 237 & 301.291 & 245 \\
\hline 71.086 & 2555 & 120.094 & 2034 & 162.969 & 4 & 217.195 & 302 & 311.273 & 85 \\
\hline 72.090 & 26 & 121.102 & 3617 & 163.148 & 1310 & 219.212 & 144 & 313.289 & 142 \\
\hline 73.029 & 108 & 122.109 & 1089 & 165.164 & 185 & 227.180 & 972 & 314.299 & 74 \\
\hline 73.047 & 282 & 123.082 & 8 & 169.102 & 85 & 228.188 & 1468 & 315.034 & 31 \\
\hline 77.039 & 344 & 123.117 & 2004 & 170.105 & 65 & 229.196 & 3529 & 326.298 & 525 \\
\hline 78.045 & 60 & 124.122 & 265 & 171.117 & 1170 & 230.201 & 749 & 327.305 & 180 \\
\hline 79.055 & 1678 & 125.095 & 82 & \begin{tabular}{|l|}
172.124 \\
\end{tabular} & 395 & 231.210 & 145 & 339.255 & 13 \\
\hline 80.062 & 67 & 125.133 & 266 & \begin{tabular}{|l|l|}
173.133 \\
\end{tabular} & 1761 & 233.227 & 246 & 339.309 & 102 \\
\hline 81.070 & 4004 & 125.766 & 71 & 174.140 & 390 & 239.180 & 207 & 353.267 & 52 \\
\hline 82.077 & 66 & 128.062 & 232 & 175.149 & 1205 & 239.237 & 124 & 353.322 & 6277 \\
\hline 83.051 & 22 & 129.070 & 604 & \begin{tabular}{|l|l|}
176.154 \\
\end{tabular} & 132 & 241.196 & 594 & 354.325 & 1775 \\
\hline 83.086 & 3563 & 129.090 & 53 & \begin{tabular}{|l|}
176.987 \\
\end{tabular} & 6 & 242.203 & 209 & 355.331 & 58 \\
\hline 84.058 & 158 & 130.078 & 184 & 177.165 & 280 & 243.212 & 379 & 367.338 & 218 \\
\hline 84.090 & 150 & 131.086 & 2599 & \begin{tabular}{|l|}
179.181 \\
\end{tabular} & 81 & 245.228 & 788 & 368.345 & 6906 \\
\hline 85.064 & 72 & 132.093 & 692 & \begin{tabular}{|l|l|}
183.117 \\
\end{tabular} & 119 & 246.234 & 496 & 368.416 & 125 \\
\hline 85.102 & 927 & 133.015 & 118 & \begin{tabular}{|l|l|}
184.126 \\
\end{tabular} & 119 & 247.243 & 2815 & 368.486 & 69 \\
\hline 87.044 & 67 & 133.102 & 3952 & 185.133 & 1342 & 248.247 & 657 & 369.351 & 4998 \\
\hline 91.054 & 2169 & 134.109 & 1515 & \begin{tabular}{|l|l|}
186.139 \\
\end{tabular} & 397 & 248.988 & 25 & 370.356 & 1036 \\
\hline 92.061 & 457 & 135.064 & 8 & \begin{tabular}{|l|l|}
187.149 \\
\end{tabular} & 1082 & 253.196 & 124 & 371.360 & 91 \\
\hline 93.070 & 2783 & 135.117 & 3243 & \begin{tabular}{|l|l|}
188.152 \\
\end{tabular} & 159 & 254.206 & 66 & 383.369 & 33 \\
\hline 94.078 & 943 & 136.124 & 940 & 189.165 & 616 & 255.212 & 12961 & 393.353 & 148 \\
\hline 95.086 & 5172 & 137.133 & 716 & \begin{tabular}{|l|l|}
191.002 \\
\end{tabular} & 49 & 255.276 & 143 & 395.368 & 426 \\
\hline 96.005 & 89 & 138.137 & 33 & \begin{tabular}{|l|l|}
191.180 \\
\end{tabular} & 270 & 255.315 & 117 & 439.064 & 56 \\
\hline 96.091 & 722 & 141.069 & 28 & 192.001 & 12 & 256.215 & 2636 & 469.406 & 101 \\
\hline 97.065 & 165 & 142.078 & 130 & \begin{tabular}{|l|l|}
193.199 \\
\end{tabular} & 154 & 257.225 & 683 & 484.429 & 64 \\
\hline 97.101 & 2015 & 143.086 & 1601 & \begin{tabular}{|l|l|}
197.133 \\
\end{tabular} & 177 & 258.241 & 73 & 511.453 & 228 \\
\hline 98.073 & 79 & 144.091 & 591 & 198.140 & 124 & 259.243 & 780 & 609.562 & 1098 \\
\hline 99.117 & 81 & 145.102 & 5023 & \begin{tabular}{|l|l|}
199.149 \\
\end{tabular} & 1477 & 260.251 & 2519 & 610.567 & 529 \\
\hline 101.059 & 33 & 146.108 & 1618 & 200.156 & 513 & 261.256 & 816 & 611.570 & 60 \\
\hline 104.063 & 80 & 147.117 & 7068 & 201.164 & 1815 & 262.262 & 44 & 624.587 & 3228 \\
\hline 105.070 & 3872 & 147.166 & 41 & 202.170 & 250 & 265.021 & 37 & 625.590 & 1409 \\
\hline 106.078 & 1359 & 148.123 & 1483 & 203.181 & 301 & 267.007 & 78 & 626.593 & 175 \\
\hline
\end{tabular}


Table S36. High-resolution mass table for the compound identified as cholesteryl heptadecanoate.

\begin{tabular}{|c|c|c|c|c|c|c|c|c|c|}
\hline Mass & $\begin{array}{c}\text { Ion } \\
\text { counts }\end{array}$ & Mass & $\begin{array}{c}\text { Ion } \\
\text { counts }\end{array}$ & Mass & $\begin{array}{c}\text { Ion } \\
\text { counts }\end{array}$ & Mass & $\begin{array}{c}\text { Ion } \\
\text { counts }\end{array}$ & Mass & $\begin{array}{c}\text { Ion } \\
\text { counts }\end{array}$ \\
\hline 53.038 & 83 & 96.005 & 369 & 131.085 & 813 & 185.133 & 249 & 253.016 & 9 \\
\hline 54.046 & 234 & 96.056 & 135 & 132.092 & 97 & 187.149 & 339 & 255.211 & 2539 \\
\hline 55.018 & 76 & 96.092 & 472 & 133.013 & 493 & 189.164 & 83 & 256.214 & 541 \\
\hline 55.054 & 2881 & 97.065 & 329 & 133.101 & 1196 & 191.002 & 812 & 257.224 & 107 \\
\hline 56.062 & 1 & 97.101 & 1492 & 134.108 & 330 & 191.179 & 236 & 259.242 & 123 \\
\hline 57.033 & 75 & 98.073 & 407 & 135.062 & 33 & 192.003 & 78 & 260.249 & 867 \\
\hline 57.070 & 3724 & 98.107 & 95 & 135.117 & 1241 & 193.015 & 43 & 261.252 & 123 \\
\hline 58.074 & 35 & 99.076 & 75 & 136.123 & 239 & 193.196 & 10 & 265.020 & 228 \\
\hline 60.021 & 169 & 99.117 & 59 & 137.133 & 248 & 197.131 & 38 & 266.022 & 86 \\
\hline 65.042 & 59 & 101.059 & 43 & 138.140 & 37 & 199.148 & 334 & 267.000 & 171 \\
\hline 67.054 & 137 & 102.968 & 73 & 143.085 & 579 & 201.164 & 562 & 281.051 & 72 \\
\hline 68.062 & 66 & 105.070 & 1212 & 144.093 & 47 & 203.179 & 81 & 282.053 & 44 \\
\hline 69.070 & 1838 & 106.077 & 415 & 145.101 & 1666 & 205.196 & 267 & 283.051 & 149 \\
\hline 70.077 & 172 & 107.086 & 1353 & 146.108 & 500 & 206.203 & 77 & 283.242 & 51 \\
\hline 71.049 & 250 & 108.093 & 444 & 147.117 & 2512 & 207.033 & 7956 & 285.246 & 83 \\
\hline 71.086 & 1723 & 109.101 & 1645 & 148.123 & 480 & 207.081 & 0 & 313.279 & 76 \\
\hline 73.029 & 217 & 110.108 & 213 & 149.133 & 877 & 208.033 & 695 & 339.256 & 36 \\
\hline 73.047 & 248 & 111.081 & 154 & 150.139 & 59 & 209.030 & 179 & 353.267 & 102 \\
\hline 77.040 & 179 & 111.117 & 578 & 151.148 & 42 & 210.030 & 30 & 353.321 & 1660 \\
\hline 78.048 & 28 & 112.088 & 156 & 157.101 & 421 & 213.164 & 1552 & 354.324 & 394 \\
\hline 79.054 & 585 & 115.002 & 29 & 158.108 & 156 & 214.168 & 383 & 355.072 & 98 \\
\hline 80.062 & 225 & 115.057 & 29 & 159.117 & 1262 & 215.178 & 367 & 355.332 & 11 \\
\hline 81.070 & 1148 & 117.070 & 401 & 160.123 & 523 & 216.183 & 38 & 367.283 & 135 \\
\hline 82.077 & 215 & 118.077 & 93 & 161.132 & 1411 & 221.085 & 14 & 368.344 & 2168 \\
\hline 83.049 & 126 & 118.997 & 6 & 162.137 & 160 & 225.220 & 45 & 369.350 & 1302 \\
\hline 83.086 & 2303 & 119.085 & 1345 & 163.148 & 415 & 227.179 & 318 & 370.354 & 155 \\
\hline 84.057 & 215 & 120.093 & 873 & 165.165 & 20 & 228.186 & 145 & 377.055 & 18 \\
\hline 84.093 & 68 & 121.101 & 1362 & 171.066 & 57 & 229.195 & 767 & 393.352 & 281 \\
\hline 85.065 & 171 & 122.108 & 329 & 171.117 & 279 & 230.201 & 36 & 394.359 & 59 \\
\hline 85.101 & 817 & 123.117 & 1022 & 172.123 & 42 & 239.239 & 108 & 395.368 & 567 \\
\hline 87.045 & 61 & 124.088 & 28 & 173.133 & 510 & 241.195 & 188 & 396.372 & 133 \\
\hline 91.054 & 646 & 124.121 & 20 & 174.139 & 113 & 243.210 & 49 & 429.094 & 39 \\
\hline 92.061 & 87 & 125.134 & 100 & 175.149 & 232 & 245.227 & 146 & 439.064 & 91 \\
\hline 93.070 & 961 & 129.070 & 166 & 176.985 & 90 & 247.243 & 959 & 638.600 & 409 \\
\hline 94.077 & 415 & 129.092 & 128 & 177.166 & 8 & 248.246 & 52 & 639.609 & 81 \\
\hline 95.086 & 1576 & 130.077 & 81 & 183.117 & 33 & 248.988 & 12 & & \\
\hline
\end{tabular}


Table S37. High-resolution mass table for the compound identified as cholesteryl oleate.

\begin{tabular}{|c|c|c|c|c|c|c|c|}
\hline Mass & $\begin{array}{c}\text { Ion } \\
\text { counts }\end{array}$ & Mass & $\begin{array}{c}\text { Ion } \\
\text { counts }\end{array}$ & Mass & $\begin{array}{c}\text { Ion } \\
\text { counts }\end{array}$ & Mass & $\begin{array}{c}\text { Ion } \\
\text { counts }\end{array}$ \\
\hline 50.005 & 31 & 100.052 & 52 & 145.101 & 5908 & 199.148 & 1169 \\
\hline 53.038 & 245 & 101.059 & 182 & 146.107 & 2098 & 200.155 & 430 \\
\hline 54.046 & 655 & 102.967 & 41 & 147.068 & 0 & 201.164 & 1098 \\
\hline 55.018 & 27 & 103.055 & 78 & 147.117 & 8533 & 202.168 & 96 \\
\hline 55.054 & 5235 & 104.062 & 188 & 147.167 & 67 & 203.180 & 322 \\
\hline 56.062 & 286 & 105.070 & 4154 & 148.122 & 1898 & 204.187 & 12 \\
\hline 57.034 & 163 & 106.077 & 1370 & 149.132 & 2539 & 205.195 & 925 \\
\hline 57.070 & 4594 & 107.085 & 4218 & 150.138 & 250 & 206.202 & 743 \\
\hline 58.073 & 44 & 108.093 & 1590 & 151.149 & 604 & 207.033 & 6753 \\
\hline 59.049 & 30 & 109.063 & 262 & 152.155 & 79 & 207.210 & 203 \\
\hline 60.021 & 156 & 109.101 & 3655 & 154.079 & 80 & 208.033 & 1047 \\
\hline 61.028 & 47 & 110.071 & 32 & 155.086 & 70 & 209.031 & 204 \\
\hline 65.038 & 54 & 110.107 & 623 & 156.095 & 79 & 211.148 & 13 \\
\hline 66.046 & 30 & 111.080 & 219 & 157.101 & 1116 & 211.203 & 52 \\
\hline 67.054 & 2234 & 111.117 & 1384 & 158.109 & 1090 & 212.156 & 16 \\
\hline 68.062 & 626 & 112.086 & 103 & 159.117 & 2940 & 213.164 & 3829 \\
\hline 69.070 & 3905 & 112.123 & 117 & 159.159 & 79 & 214.168 & 960 \\
\hline 70.078 & 577 & 113.059 & 18 & 160.124 & 1921 & 215.179 & 1115 \\
\hline 71.050 & 119 & 115.054 & 176 & 161.132 & 3303 & 216.182 & 101 \\
\hline 71.086 & 2266 & 117.070 & 1415 & 162.138 & 688 & 217.196 & 258 \\
\hline 72.090 & 46 & 118.077 & 849 & 162.970 & 11 & 218.202 & 70 \\
\hline 73.028 & 349 & 118.997 & 67 & 163.148 & 1611 & 219.212 & 480 \\
\hline 73.047 & 142 & 119.085 & 2712 & 164.155 & 92 & 220.216 & 104 \\
\hline 77.039 & 635 & 120.093 & 2652 & 165.163 & 290 & 221.085 & 44 \\
\hline 78.047 & 112 & 121.101 & 3603 & 169.102 & 103 & 227.181 & 436 \\
\hline 79.054 & 2092 & 122.108 & 1018 & 171.063 & 30 & 228.187 & 680 \\
\hline 80.061 & 592 & 123.081 & 66 & 171.117 & 929 & 229.195 & 1005 \\
\hline 81.070 & 5841 & 123.117 & 1821 & 172.123 & 375 & 230.199 & 145 \\
\hline 82.077 & 686 & 124.087 & 9 & 173.132 & 1377 & 231.210 & 86 \\
\hline 83.049 & 171 & 124.123 & 207 & 174.140 & 366 & 233.226 & 79 \\
\hline 83.086 & 4120 & 125.096 & 156 & 175.148 & 1327 & 236.206 & 89 \\
\hline 84.057 & 505 & 125.132 & 429 & 176.153 & 232 & 239.236 & 102 \\
\hline 84.092 & 446 & 128.062 & 229 & 176.985 & 64 & 241.196 & 268 \\
\hline 85.064 & 255 & 129.069 & 893 & 177.164 & 245 & 243.210 & 291 \\
\hline 85.101 & 699 & 129.090 & 273 & 179.180 & 168 & 245.226 & 910 \\
\hline 87.044 & 142 & 130.076 & 274 & 183.118 & 61 & 246.233 & 647 \\
\hline 89.994 & 27 & 131.085 & 2597 & 185.132 & 916 & 247.242 & 4545 \\
\hline 91.054 & 2540 & 132.093 & 522 & 186.139 & 255 & 248.246 & 1146 \\
\hline 92.061 & 813 & 133.013 & 265 & 187.148 & 771 & 248.986 & 73 \\
\hline 93.070 & 3163 & 133.101 & 3152 & 188.153 & 103 & 253.016 & 13 \\
\hline 94.077 & 1029 & 134.108 & 932 & 189.164 & 434 & 253.195 & 121 \\
\hline 95.085 & 5307 & 135.064 & 30 & 190.173 & 69 & 255.211 & 4543 \\
\hline 95.113 & 1134 & 135.117 & 3188 & 191.001 & 490 & 256.215 & 1141 \\
\hline 96.005 & 87 & 136.122 & 716 & 191.179 & 170 & 257.226 & 404 \\
\hline 96.057 & 8 & 137.096 & 61 & 191.999 & 57 & 258.232 & 63 \\
\hline 96.091 & 936 & 137.132 & 831 & 192.185 & 1 & 259.242 & 860 \\
\hline 97.065 & 329 & 139.111 & 35 & 193.015 & 33 & 260.250 & 3718 \\
\hline 97.101 & 2638 & 139.148 & 50 & 193.195 & 441 & 261.255 & 1234 \\
\hline 98.073 & 474 & 141.070 & 171 & 194.199 & 44 & 262.266 & 60 \\
\hline 98.106 & 21 & 142.078 & 219 & 197.078 & 42 & 265.018 & 133 \\
\hline 99.077 & 58 & 143.085 & 2522 & 197.136 & 53 & 267.002 & 68 \\
\hline 99.117 & 84 & 144.092 & 868 & 198.138 & 95 & 273.257 & 113 \\
\hline
\end{tabular}


Table S37 (continued). High-resolution mass table for the compound identified as cholesteryl oleate.

\begin{tabular}{|c|c|c|c|c|c|c|c|}
\hline Mass & $\begin{array}{c}\text { Ion } \\
\text { counts }\end{array}$ & Mass & $\begin{array}{c}\text { Ion } \\
\text { counts }\end{array}$ & Mass & $\begin{array}{c}\text { Ion } \\
\text { counts }\end{array}$ & Mass & $\begin{array}{c}\text { Ion } \\
\text { counts }\end{array}$ \\
\hline 274.265 & 385 & 297.279 & 97 & 341.016 & 40 & 368.344 & 10274 \\
\hline 275.273 & 158 & 299.274 & 122 & 351.305 & 113 & 368.407 & 268 \\
\hline 280.239 & 7 & 301.289 & 196 & 353.271 & 22 & 369.349 & 7133 \\
\hline 281.052 & 703 & 311.273 & 139 & 353.320 & 6957 & 369.426 & 139 \\
\hline 282.052 & 55 & 313.283 & 169 & 354.324 & 1926 & 370.354 & 1609 \\
\hline 283.049 & 120 & 314.296 & 56 & 355.071 & 42 & 371.361 & 247 \\
\hline 283.242 & 475 & 315.033 & 34 & 355.332 & 184 & 377.048 & 53 \\
\hline 284.246 & 106 & 326.296 & 785 & 366.325 & 76 & 439.062 & 51 \\
\hline 285.251 & 113 & 327.301 & 220 & 367.284 & 278 & 523.470 & 86 \\
\hline 287.275 & 341 & 339.307 & 145 & 367.336 & 245 & 650.596 & 174 \\
\hline 297.254 & 77 & 340.314 & 81 & 368.292 & 105 & & \\
\hline
\end{tabular}


Table S38. High-resolution mass table for the compound identified as cholesteryl stearate.

\begin{tabular}{|c|c|c|c|c|c|c|c|c|c|}
\hline Mass & $\begin{array}{c}\text { Ion } \\
\text { counts }\end{array}$ & Mass & $\begin{array}{c}\text { Ion } \\
\text { counts }\end{array}$ & Mass & $\begin{array}{c}\text { Ion } \\
\text { counts }\end{array}$ & Mass & $\begin{array}{c}\text { Ion } \\
\text { counts }\end{array}$ & Mass & $\begin{array}{c}\text { Ion } \\
\text { counts }\end{array}$ \\
\hline 53.039 & 132 & 96.092 & 635 & 133.101 & 1360 & \begin{tabular}{|l|}
192.977 \\
\end{tabular} & 59 & 266.024 & 54 \\
\hline 54.047 & 321 & 96.503 & 20 & 134.108 & 597 & \begin{tabular}{|l|}
193.011 \\
\end{tabular} & 37 & 267.001 & 65 \\
\hline 55.018 & 392 & 97.003 & 14 & 135.063 & 14 & \begin{tabular}{|l|}
193.194 \\
\end{tabular} & 35 & 269.241 & 64 \\
\hline 55.054 & 3503 & 97.065 & 581 & 135.117 & 1486 & \begin{tabular}{|l|}
197.077 \\
\end{tabular} & 76 & 281.051 & 159 \\
\hline 56.062 & 82 & 97.101 & 1947 & 136.123 & 383 & \begin{tabular}{|l|}
199.148 \\
\end{tabular} & 502 & 282.052 & 110 \\
\hline 57.033 & 36 & 98.073 & 765 & 137.133 & 475 & 200.155 & 226 & 283.049 & 292 \\
\hline 57.070 & 4266 & 98.107 & 131 & 138.139 & 76 & 201.163 & 617 & 283.247 & 64 \\
\hline 58.073 & 21 & 99.076 & 59 & 143.085 & 619 & 203.180 & 73 & 285.251 & 71 \\
\hline 59.037 & 79 & 99.116 & 85 & 144.092 & 115 & 207.032 & 9688 & 287.274 & 259 \\
\hline 60.020 & 171 & 100.051 & 9 & 145.101 & 1864 & \begin{tabular}{|l|}
207.074 \\
\end{tabular} & 21 & 297.280 & 20 \\
\hline 61.029 & 12 & 101.059 & 52 & 146.107 & 474 & 207.212 & 37 & 299.261 & 157 \\
\hline 65.039 & 34 & 105.070 & 1688 & 147.117 & 2464 & 208.033 & 1309 & 301.291 & 128 \\
\hline 66.049 & 35 & 106.077 & 588 & 148.123 & 491 & \begin{tabular}{|l|l|}
209.030 \\
\end{tabular} & 50 & 313.275 & 163 \\
\hline 67.054 & 649 & 107.086 & 1901 & 149.132 & 1285 & \begin{tabular}{|l|l|}
213.163 \\
\end{tabular} & 2192 & 315.032 & 7 \\
\hline 68.062 & 88 & 108.092 & 472 & 151.147 & 234 & 213.184 & 137 & 326.296 & 103 \\
\hline 69.034 & 1044 & 109.101 & 2185 & 152.155 & 122 & 214.168 & 277 & 339.253 & 143 \\
\hline 69.070 & 3132 & 110.073 & 37 & 157.101 & 552 & \begin{tabular}{|l|l|}
215.179 \\
\end{tabular} & 533 & 353.269 & 597 \\
\hline 70.077 & 330 & 110.107 & 265 & 158.057 & 27 & \begin{tabular}{|l|l|}
216.182 \\
\end{tabular} & 56 & 353.320 & 1556 \\
\hline 71.049 & 136 & 111.080 & 236 & 158.109 & 221 & 217.195 & 124 & 354.324 & 321 \\
\hline 71.086 & 1963 & 111.117 & 747 & 159.117 & 1389 & \begin{tabular}{|l|}
219.211 \\
\end{tabular} & 277 & 355.071 & 14 \\
\hline 72.090 & 40 & 112.088 & 68 & 160.124 & 467 & 221.084 & 0 & 365.268 & 123 \\
\hline 73.029 & 345 & 113.060 & 33 & 161.132 & 2001 & 225.220 & 10 & 367.284 & 570 \\
\hline 73.047 & 113 & 115.058 & 49 & 162.138 & 309 & \begin{tabular}{|l|l|}
227.180 \\
\end{tabular} & 309 & 367.335 & 157 \\
\hline 77.038 & 209 & 115.073 & 67 & 162.970 & 5 & 228.188 & 260 & 368.288 & 93 \\
\hline 78.046 & 33 & 117.070 & 426 & 163.148 & 417 & 229.195 & 1263 & 368.344 & 1735 \\
\hline 79.054 & 1056 & 118.077 & 170 & 164.983 & 61 & 230.199 & 145 & 369.351 & 3568 \\
\hline 80.062 & 262 & 118.997 & 47 & 165.164 & 69 & 233.226 & 8 & 370.355 & 1007 \\
\hline 81.070 & 2500 & 119.085 & 1746 & 169.101 & 54 & 236.212 & 93 & 377.047 & 48 \\
\hline 82.077 & 28 & 120.092 & 758 & 171.065 & 268 & 237.218 & 72 & 381.296 & 47 \\
\hline 83.049 & 136 & 121.101 & 1773 & 171.117 & 391 & 239.238 & 197 & 395.367 & 309 \\
\hline 83.086 & 3167 & 122.107 & 457 & 171.138 & 92 & 241.195 & 106 & 407.311 & 61 \\
\hline 84.057 & 826 & 123.079 & 34 & 172.124 & 54 & 243.212 & 273 & 439.064 & 14 \\
\hline 84.091 & 316 & 123.117 & 1162 & 173.133 & 789 & 245.225 & 158 & 440.071 & 29 \\
\hline 85.065 & 252 & 124.123 & 135 & 174.139 & 50 & 247.243 & 776 & 535.469 & 100 \\
\hline 85.101 & 884 & 125.096 & 79 & 175.148 & 592 & 248.245 & 22 & 537.479 & 160 \\
\hline 87.044 & 72 & 125.132 & 179 & 176.984 & 92 & 248.988 & 62 & 538.486 & 82 \\
\hline 91.054 & 871 & 128.063 & 10 & 177.164 & 22 & \begin{tabular}{|l|}
253.195 \\
\end{tabular} & 28 & 635.578 & 105 \\
\hline 92.061 & 86 & 129.052 & 44 & 185.133 & 402 & 255.211 & 3421 & 636.578 & 28 \\
\hline 93.070 & 1200 & 129.069 & 61 & 186.137 & 67 & 256.215 & 772 & 650.602 & 100 \\
\hline 94.077 & 522 & 129.091 & 225 & 187.149 & 414 & 257.224 & 268 & 652.615 & 244 \\
\hline 95.049 & 1026 & 130.076 & 64 & 189.165 & 255 & 259.242 & 249 & & \\
\hline 95.085 & 2813 & 131.086 & 1028 & 191.001 & 582 & 260.249 & 531 & & \\
\hline 96.005 & 435 & 132.092 & 215 & 191.180 & 650 & 261.255 & 155 & & \\
\hline 96.057 & 18 & 133.013 & 490 & 192.003 & 53 & 265.020 & 120 & & \\
\hline
\end{tabular}


Table S39. High-resolution mass table for the compound identified as tripalmitin.

\begin{tabular}{|c|c|c|c|c|c|c|c|}
\hline Mass & $\begin{array}{c}\text { Ion } \\
\text { counts }\end{array}$ & Mass & $\begin{array}{c}\text { Ion } \\
\text { counts }\end{array}$ & Mass & $\begin{array}{c}\text { Ion } \\
\text { counts }\end{array}$ & Mass & $\begin{array}{c}\text { Ion } \\
\text { counts }\end{array}$ \\
\hline 50.017 & 1331 & 61.013 & 742 & 78.049 & 5521 & 89.044 & 505 \\
\hline 51.026 & 2106 & 61.031 & 6715 & 79.057 & 17865 & 91.057 & 8124 \\
\hline 52.033 & 1505 & 62.019 & 336 & 80.064 & 13348 & 92.064 & 2785 \\
\hline 53.041 & 7507 & 63.025 & 733 & 80.983 & 389 & 93.072 & 14434 \\
\hline 54.049 & 21226 & 65.041 & 2853 & 81.036 & 3143 & 93.139 & 383 \\
\hline 54.088 & 364 & 66.049 & 3245 & 81.072 & 65868 & 94.043 & 705 \\
\hline 54.110 & 350 & 67.057 & 57492 & 81.361 & 424 & 94.080 & 10282 \\
\hline 54.143 & 396 & 67.354 & 227 & 81.396 & 436 & 94.157 & 399 \\
\hline 55.020 & 52241 & 67.386 & 507 & 81.420 & 237 & 95.052 & 4503 \\
\hline 55.057 & 130001 & 67.647 & 400 & 81.466 & 516 & 95.088 & 71808 \\
\hline 55.118 & 4133 & 68.028 & 1772 & 81.485 & 411 & 95.441 & 513 \\
\hline 55.325 & 1548 & 68.064 & 23706 & 81.696 & 476 & 95.468 & 387 \\
\hline 55.378 & 920 & 69.036 & 6840 & 81.743 & 476 & 95.498 & 369 \\
\hline 55.537 & 470 & 69.072 & 116816 & 81.992 & 581 & 95.781 & 431 \\
\hline 55.577 & 1177 & 69.344 & 504 & 82.044 & 3144 & 95.821 & 404 \\
\hline 55.761 & 388 & 69.373 & 795 & 82.080 & 27880 & 96.007 & 5280 \\
\hline 55.809 & 771 & 69.409 & 1003 & 82.152 & 587 & 96.059 & 7452 \\
\hline 56.027 & 5203 & 69.484 & 280 & 83.052 & 20808 & 96.095 & 23145 \\
\hline 56.064 & 35201 & 69.649 & 649 & 83.088 & 91758 & 96.159 & 931 \\
\hline 56.122 & 1435 & 69.689 & 669 & 83.135 & 1314 & 96.409 & 426 \\
\hline 56.301 & 477 & 69.913 & 432 & 83.162 & 2789 & 96.506 & 1397 \\
\hline 56.337 & 523 & 69.956 & 466 & 83.387 & 591 & 97.005 & 823 \\
\hline 56.590 & 480 & 70.044 & 5842 & 83.418 & 607 & 97.067 & 34878 \\
\hline 56.617 & 315 & 70.079 & 25253 & 83.454 & 797 & 97.104 & 57745 \\
\hline 57.036 & 17732 & 70.185 & 548 & 83.716 & 341 & 97.184 & 2149 \\
\hline 57.072 & 219294 & 70.247 & 356 & 83.749 & 626 & 97.417 & 557 \\
\hline 57.113 & 2166 & 70.430 & 353 & 84.059 & 83074 & 97.462 & 928 \\
\hline 57.279 & 361 & 71.052 & 8973 & 84.094 & 13066 & 97.772 & 550 \\
\hline 57.320 & 1643 & 71.088 & 126637 & 84.362 & 716 & 97.842 & 420 \\
\hline 57.345 & 1435 & 71.133 & 1348 & 84.392 & 522 & 98.038 & 1141 \\
\hline 57.368 & 1862 & 71.366 & 519 & 84.432 & 651 & 98.075 & 95042 \\
\hline 57.396 & 1748 & 71.394 & 848 & 84.701 & 586 & 98.109 & 7460 \\
\hline 57.448 & 393 & 71.418 & 755 & 85.031 & 1804 & 98.390 & 580 \\
\hline 57.604 & 1820 & 71.456 & 847 & 85.066 & 12986 & 98.432 & 706 \\
\hline 57.642 & 1488 & 71.684 & 690 & 85.104 & 75744 & 98.470 & 1240 \\
\hline 57.859 & 1298 & 71.955 & 484 & 85.403 & 493 & 98.752 & 636 \\
\hline 57.920 & 349 & 71.996 & 432 & 85.438 & 285 & 98.776 & 571 \\
\hline 58.044 & 5585 & 72.058 & 2112 & 85.468 & 487 & 98.822 & 557 \\
\hline 58.076 & 10038 & 72.092 & 6729 & 85.517 & 449 & 99.046 & 2633 \\
\hline 58.115 & 921 & 72.227 & 413 & 85.777 & 323 & 99.080 & 11662 \\
\hline 58.330 & 532 & 73.031 & 32427 & 85.817 & 370 & 99.119 & 12681 \\
\hline 58.358 & 431 & 73.051 & 1895 & 86.039 & 968 & 99.166 & 825 \\
\hline 58.379 & 506 & 73.067 & 2559 & 86.072 & 1676 & 99.391 & 454 \\
\hline 58.536 & 262 & 73.340 & 368 & 86.107 & 5224 & 99.437 & 394 \\
\hline 58.572 & 466 & 73.377 & 419 & 87.002 & 577 & 99.470 & 491 \\
\hline 58.611 & 445 & 73.411 & 407 & 87.046 & 6903 & 99.775 & 482 \\
\hline 59.036 & 1077 & 74.038 & 5024 & 87.083 & 905 & 100.054 & 6047 \\
\hline 59.052 & 4292 & 75.028 & 2397 & 87.112 & 398 & 100.088 & 1673 \\
\hline 59.087 & 413 & 75.046 & 1544 & 87.992 & 299 & 100.124 & 1326 \\
\hline 60.023 & 24827 & 76.032 & 514 & 88.054 & 1108 & 100.469 & 386 \\
\hline 60.055 & 383 & 77.012 & 360 & 88.954 & 467 & 101.062 & 5286 \\
\hline 60.086 & 719 & 77.041 & 4710 & 88.999 & 336 & 101.098 & 680 \\
\hline
\end{tabular}


Table S39 (continued). High-resolution mass table for the compound identified as tripalmitin.

\begin{tabular}{|c|c|c|c|c|c|c|c|}
\hline Mass & $\begin{array}{c}\text { Ion } \\
\text { counts }\end{array}$ & Mass & $\begin{array}{c}\text { Ion } \\
\text { counts }\end{array}$ & Mass & $\begin{array}{c}\text { Ion } \\
\text { counts }\end{array}$ & Mass & $\begin{array}{c}\text { Ion } \\
\text { counts }\end{array}$ \\
\hline 102.035 & 1397 & 121.065 & 1126 & 140.085 & 1014 & 161.134 & 2145 \\
\hline 102.068 & 1179 & 121.103 & 16703 & 140.120 & 1682 & 162.141 & 840 \\
\hline 102.969 & 1293 & 121.189 & 469 & 140.157 & 981 & 162.971 & 1397 \\
\hline 103.053 & 822 & 122.075 & 721 & 141.086 & 1345 & 163.150 & 2863 \\
\hline 103.969 & 280 & 122.109 & 4543 & \begin{tabular}{|l|l|}
141.129 \\
\end{tabular} & 5184 & 163.979 & 357 \\
\hline 104.064 & 801 & 123.083 & 14086 & \begin{tabular}{|l|l|}
141.167 \\
\end{tabular} & 1554 & 164.121 & 758 \\
\hline 104.988 & 417 & 123.119 & 18112 & \begin{tabular}{|l|}
142.073 \\
\end{tabular} & 765 & 164.157 & 1375 \\
\hline 105.029 & 446 & 123.180 & 650 & 142.134 & 914 & 164.986 & 865 \\
\hline 105.072 & 4883 & 123.222 & 586 & 143.076 & 1925 & 165.072 & 807 \\
\hline 106.079 & 2354 & 124.090 & 4997 & 143.108 & 1970 & 165.129 & 3015 \\
\hline 107.051 & 929 & 124.126 & 6929 & 143.144 & 436 & 165.165 & 2375 \\
\hline 107.088 & 10323 & 125.062 & 644 & \begin{tabular}{|l|}
144.097 \\
\end{tabular} & 658 & 166.136 & 2081 \\
\hline 108.058 & 562 & 125.098 & 8616 & 145.102 & 1608 & 166.172 & 3188 \\
\hline 108.095 & 6706 & 125.135 & 6223 & \begin{tabular}{|l|}
146.067 \\
\end{tabular} & 356 & 167.144 & 2332 \\
\hline 109.067 & 4061 & 126.070 & 1207 & \begin{tabular}{|l|l|}
146.109 \\
\end{tabular} & 572 & 167.180 & 1187 \\
\hline 109.103 & 39498 & 126.105 & 4107 & 146.942 & 1086 & 168.114 & 548 \\
\hline 109.526 & 434 & 126.141 & 1689 & 147.072 & 1153 & 168.151 & 1313 \\
\hline 110.075 & 12573 & 127.045 & 794 & 147.118 & 3257 & 169.089 & 845 \\
\hline 110.110 & 10666 & 127.077 & 1953 & \begin{tabular}{|l|}
148.125 \\
\end{tabular} & 2107 & 169.125 & 1397 \\
\hline 110.169 & 598 & 127.114 & 3381 & 148.944 & 375 & 169.160 & 2241 \\
\hline 110.223 & 496 & 127.150 & 2868 & 149.053 & 499 & 170.060 & 1051 \\
\hline 111.047 & 1719 & 128.065 & 1087 & 149.098 & 860 & 170.167 & 557 \\
\hline 111.083 & 23361 & 128.118 & 709 & 149.134 & 6029 & 171.067 & 26110 \\
\hline 111.119 & 18770 & 128.153 & 304 & 150.106 & 982 & 171.111 & 1352 \\
\hline 111.174 & 1229 & 129.057 & 15371 & 150.141 & 2195 & 171.140 & 3610 \\
\hline 111.535 & 438 & 129.093 & 9156 & 151.114 & 5807 & 171.248 & 511 \\
\hline 112.053 & 1374 & 129.134 & 1055 & 151.150 & 4277 & 172.072 & 4175 \\
\hline 112.090 & 26000 & 129.213 & 454 & 152.065 & 1024 & 172.122 & 1143 \\
\hline 112.126 & 3153 & 130.062 & 1990 & 152.121 & 5665 & 173.075 & 670 \\
\hline 112.181 & 1069 & 130.096 & 1161 & 152.158 & 15799 & 173.133 & 662 \\
\hline 112.231 & 621 & 131.087 & 2005 & 152.246 & 526 & 175.149 & 1388 \\
\hline 112.560 & 413 & 132.007 & 277 & \begin{tabular}{|l|}
153.070 \\
\end{tabular} & 1086 & 175.979 & 428 \\
\hline 113.062 & 6080 & 132.094 & 685 & 153.129 & 3487 & 176.158 & 527 \\
\hline 113.097 & 5224 & 132.926 & 425 & 153.164 & 3994 & 176.986 & 2583 \\
\hline 113.135 & 5691 & 133.016 & 5160 & 154.081 & 2569 & 177.166 & 1840 \\
\hline 113.202 & 518 & 133.103 & 4392 & 154.136 & 1876 & 177.988 & 665 \\
\hline 114.069 & 1977 & 134.017 & 893 & 154.169 & 769 & 178.084 & 458 \\
\hline 114.104 & 795 & 134.110 & 3375 & 155.094 & 1699 & 178.171 & 1229 \\
\hline 114.138 & 596 & 135.012 & 530 & 155.144 & 3524 & 178.996 & 986 \\
\hline 115.004 & 1005 & 135.066 & 3657 & 155.182 & 972 & 179.085 & 399 \\
\hline 115.050 & 2366 & 135.119 & 12847 & 156.010 & 413 & 179.144 & 1806 \\
\hline 115.078 & 4738 & 135.206 & 479 & 156.151 & 786 & 179.179 & 1254 \\
\hline 115.114 & 967 & 136.089 & 1852 & 157.013 & 488 & 180.003 & 458 \\
\hline 116.049 & 8512 & 136.125 & 3518 & \begin{tabular}{|l}
157.091 \\
\end{tabular} & 1435 & 180.151 & 1300 \\
\hline 116.086 & 1402 & 137.045 & 1200 & 157.124 & 2397 & 180.188 & 1944 \\
\hline 116.125 & 456 & 137.098 & 11843 & 158.020 & 415 & 181.014 & 1186 \\
\hline 116.990 & 383 & 137.135 & 9223 & 158.059 & 14833 & 181.159 & 1894 \\
\hline 117.057 & 5920 & 138.105 & 4690 & 158.129 & 970 & 182.167 & 1128 \\
\hline 118.078 & 911 & 138.142 & 7106 & 159.065 & 2555 & 183.067 & 4310 \\
\hline 118.999 & 1683 & 138.205 & 395 & 159.119 & 905 & 183.175 & 1297 \\
\hline 119.088 & 5611 & 139.113 & 5235 & 160.126 & 470 & 184.071 & 1106 \\
\hline 120.094 & 2590 & 139.149 & 3043 & 160.956 & 719 & 184.166 & 436 \\
\hline
\end{tabular}


Table S39 (continued). High-resolution mass table for the compound identified as tripalmitin.

\begin{tabular}{|c|c|c|c|c|c|c|c|}
\hline Mass & $\begin{array}{c}\text { Ion } \\
\text { counts }\end{array}$ & Mass & $\begin{array}{c}\text { Ion } \\
\text { counts }\end{array}$ & Mass & $\begin{array}{c}\text { Ion } \\
\text { counts }\end{array}$ & Mass & $\begin{array}{c}\text { Ion } \\
\text { counts }\end{array}$ \\
\hline 185.083 & 5619 & 210.031 & 1534 & \begin{tabular}{|l|}
239.997 \\
\end{tabular} & 433 & 268.167 & 376 \\
\hline 185.119 & 2958 & 210.197 & 1218 & 240.139 & 320 & 268.270 & 960 \\
\hline 185.155 & 3850 & 211.024 & 610 & 240.241 & 13723 & 269.047 & 873 \\
\hline 186.087 & 1186 & 211.098 & 2054 & 240.348 & 1064 & 269.176 & 4792 \\
\hline 186.123 & 515 & \begin{tabular}{|l|}
211.206 \\
\end{tabular} & 14935 & \begin{tabular}{|l|}
240.409 \\
\end{tabular} & 649 & 270.180 & 1783 \\
\hline 186.158 & 576 & 211.320 & 487 & \begin{tabular}{|l|}
240.857 \\
\end{tabular} & 582 & 271.238 & 695 \\
\hline 187.107 & 445 & 212.105 & 503 & 241.011 & 497 & 277.244 & 464 \\
\hline 187.149 & 422 & 212.211 & 2475 & 241.144 & 2447 & 278.258 & 756 \\
\hline 189.024 & 419 & 213.113 & 4331 & \begin{tabular}{|l|}
241.180 \\
\end{tabular} & 1319 & 279.255 & 895 \\
\hline 189.166 & 728 & 213.150 & 1202 & 241.243 & 2623 & 280.239 & 2141 \\
\hline 190.171 & 475 & 213.185 & 3993 & 242.187 & 1512 & 281.051 & 16589 \\
\hline 191.002 & 7251 & 214.121 & 1248 & 243.231 & 876 & 281.175 & 1851 \\
\hline 191.107 & 491 & 214.173 & 1431 & 245.227 & 748 & 281.246 & 2163 \\
\hline 191.181 & 1715 & 217.197 & 1592 & 246.234 & 1487 & 282.050 & 4703 \\
\hline 192.002 & 1611 & 218.023 & 706 & 247.243 & 1848 & 282.251 & 777 \\
\hline 192.189 & 3304 & 218.203 & 2765 & 248.246 & 683 & 283.049 & 3200 \\
\hline 193.005 & 3216 & 218.526 & 314 & 248.989 & 1691 & 283.190 & 6130 \\
\hline 193.160 & 2426 & 219.029 & 720 & 249.220 & 1296 & 284.047 & 642 \\
\hline 193.193 & 1850 & 219.211 & 6646 & 249.990 & 485 & 284.196 & 2510 \\
\hline 194.011 & 614 & 220.217 & 2530 & 250.229 & 4690 & 284.988 & 474 \\
\hline 194.167 & 1934 & 221.085 & 556 & 250.992 & 540 & 285.242 & 13319 \\
\hline 194.203 & 2353 & 221.190 & 2773 & 251.236 & 3253 & 286.245 & 2618 \\
\hline 195.029 & 902 & 221.226 & 2169 & 251.946 & 419 & 287.246 & 325 \\
\hline 195.064 & 1553 & 222.198 & 2791 & 252.242 & 1595 & 292.276 & 535 \\
\hline 195.176 & 2560 & 223.205 & 2036 & 253.017 & 3810 & 293.247 & 898 \\
\hline 196.047 & 561 & 224.214 & 737 & 253.144 & 1640 & 294.258 & 687 \\
\hline 196.183 & 854 & 225.112 & 1825 & 253.252 & 5575 & 295.189 & 897 \\
\hline 197.044 & 1078 & 225.222 & 9415 & 254.020 & 1026 & 295.265 & 1454 \\
\hline 197.081 & 3967 & 226.116 & 517 & 254.147 & 531 & 296.268 & 656 \\
\hline 197.156 & 511 & 226.225 & 1702 & 254.256 & 1387 & 297.207 & 4908 \\
\hline 197.188 & 1048 & 227.129 & 10106 & 255.020 & 1221 & 297.276 & 2046 \\
\hline 198.086 & 1225 & 227.201 & 2103 & 255.159 & 2782 & 298.214 & 1185 \\
\hline 199.096 & 1758 & 228.133 & 1646 & 255.198 & 2145 & 298.270 & 615 \\
\hline 199.134 & 1219 & 228.170 & 1305 & 256.168 & 1007 & 298.995 & 217 \\
\hline 199.169 & 1663 & 229.216 & 873 & 256.238 & 1474 & 299.257 & 9894 \\
\hline 200.119 & 618 & 231.213 & 302 & 257.057 & 1221 & 300.261 & 2168 \\
\hline 201.102 & 408 & 232.222 & 449 & 257.248 & 2183 & 303.257 & 400 \\
\hline 201.163 & 531 & 233.224 & 902 & 258.063 & 453 & 305.271 & 464 \\
\hline 203.181 & 405 & 234.198 & 2243 & 258.246 & 406 & 306.308 & 551 \\
\hline 205.020 & 503 & 235.205 & 3708 & 259.093 & 656 & 307.288 & 572 \\
\hline 205.198 & 1005 & 236.214 & 47361 & 261.250 & 412 & 308.269 & 830 \\
\hline 206.204 & 1341 & 236.332 & 1732 & 262.228 & 2325 & 309.241 & 2857 \\
\hline 207.033 & 58076 & 236.795 & 529 & 263.236 & 2065 & 310.248 & 2217 \\
\hline 207.175 & 4585 & 237.220 & 26164 & 264.245 & 14891 & 311.220 & 4549 \\
\hline 207.209 & 2198 & 237.350 & 1286 & 265.020 & 2859 & 311.255 & 12734 \\
\hline 207.558 & 544 & 238.227 & 7181 & 265.251 & 9788 & 311.377 & 568 \\
\hline 207.663 & 464 & 238.325 & 513 & 266.020 & 819 & 312.261 & 5062 \\
\hline 208.034 & 12751 & 239.002 & 481 & 266.255 & 2277 & 313.011 & 692 \\
\hline 208.184 & 4347 & 239.128 & 1998 & 267.015 & 1131 & 313.272 & 86932 \\
\hline 209.031 & 7516 & 239.237 & 77854 & 267.159 & 1670 & 313.403 & 2071 \\
\hline 209.088 & 854 & 239.354 & 3364 & 267.267 & 4200 & 313.434 & 2549 \\
\hline 209.190 & 2874 & 239.830 & 768 & 268.041 & 443 & 313.927 & 607 \\
\hline
\end{tabular}


Table S39 (continued). High-resolution mass table for the compound identified as tripalmitin.

\begin{tabular}{|c|c|c|c|c|c|c|c|}
\hline Mass & $\begin{array}{c}\text { Ion } \\
\text { counts }\end{array}$ & Mass & $\begin{array}{c}\text { Ion } \\
\text { counts }\end{array}$ & Mass & $\begin{array}{c}\text { Ion } \\
\text { counts }\end{array}$ & Mass & $\begin{array}{c}\text { Ion } \\
\text { counts }\end{array}$ \\
\hline 314.018 & 1062 & 369.289 & 4140 & 448.446 & 387 & 524.469 & 9534 \\
\hline 314.276 & 17628 & 370.295 & 449 & \begin{tabular}{|l|}
449.360 \\
\end{tabular} & 4861 & 525.473 & 1963 \\
\hline 314.415 & 703 & 373.020 & 622 & 450.366 & 1442 & 529.106 & 497 \\
\hline 314.502 & 841 & 375.031 & 448 & 451.064 & 1360 & 530.109 & 339 \\
\hline 315.032 & 5731 & 377.045 & 7200 & \begin{tabular}{|l|}
451.373 \\
\end{tabular} & 8660 & 533.450 & 2182 \\
\hline 315.278 & 2599 & 377.381 & 585 & 452.068 & 601 & 534.459 & 1169 \\
\hline 316.033 & 2024 & 378.048 & 2613 & 452.379 & 4371 & 535.465 & 4300 \\
\hline 316.281 & 586 & 379.046 & 1293 & 453.064 & 436 & 536.472 & 2400 \\
\hline 317.037 & 1470 & 379.282 & 2693 & \begin{tabular}{|l|}
453.382 \\
\end{tabular} & 1189 & 537.481 & 9457 \\
\hline 318.046 & 497 & 379.354 & 976 & 455.089 & 1779 & 538.484 & 3545 \\
\hline 319.045 & 410 & 380.290 & 1638 & \begin{tabular}{|l|l|}
456.091 \\
\end{tabular} & 845 & 539.489 & 774 \\
\hline 320.303 & 501 & 381.298 & 12342 & 463.375 & 2636 & 546.462 & 554 \\
\hline 321.284 & 562 & 381.448 & 570 & 464.379 & 1236 & 547.466 & 4389 \\
\hline 323.229 & 933 & 382.303 & 3862 & 465.390 & 6607 & 548.473 & 9385 \\
\hline 323.314 & 443 & 383.305 & 1300 & 466.395 & 4429 & 549.481 & 37060 \\
\hline 324.260 & 504 & 389.049 & 658 & 467.083 & 429 & 549.668 & 1133 \\
\hline 325.236 & 4890 & 391.058 & 477 & 467.401 & 1412 & 550.486 & 21383 \\
\hline 326.243 & 2669 & 391.283 & 295 & 468.096 & 412 & 551.307 & 667 \\
\hline 327.035 & 524 & 392.067 & 856 & 470.116 & 1036 & 551.496 & 78583 \\
\hline 327.287 & 8775 & 392.367 & 487 & 471.111 & 444 & 551.684 & 3412 \\
\hline 328.292 & 1970 & 393.078 & 689 & 474.450 & 449 & 552.500 & 29386 \\
\hline 331.063 & 1491 & 393.297 & 9022 & 475.445 & 1008 & 552.682 & 999 \\
\hline 331.285 & 2076 & 394.076 & 448 & 476.452 & 516 & 553.503 & 5951 \\
\hline 332.065 & 445 & 394.300 & 2672 & 477.390 & 2290 & 554.510 & 858 \\
\hline 332.288 & 501 & 395.312 & 9977 & 477.461 & 1658 & 559.062 & 494 \\
\hline 334.303 & 486 & 396.317 & 3202 & 478.395 & 1256 & 561.484 & 1278 \\
\hline 336.274 & 528 & 397.323 & 743 & 479.405 & 6952 & 562.490 & 2327 \\
\hline 337.265 & 2606 & 405.084 & 390 & 480.412 & 5185 & 563.496 & 8649 \\
\hline 338.277 & 1780 & 407.313 & 7008 & 481.415 & 1760 & 563.682 & 628 \\
\hline 339.252 & 17282 & 408.316 & 2275 & 491.405 & 2152 & 564.501 & 4110 \\
\hline 340.256 & 4757 & 409.328 & 9961 & 492.408 & 821 & 565.512 & 9369 \\
\hline 341.302 & 6079 & 410.333 & 3359 & 493.421 & 4276 & 566.515 & 3700 \\
\hline 342.306 & 1078 & 411.336 & 691 & 494.426 & 2480 & 567.520 & 1052 \\
\hline 347.308 & 515 & 411.412 & 565 & 495.431 & 966 & 575.099 & 790 \\
\hline 348.328 & 384 & 419.322 & 575 & 497.043 & 599 & 575.494 & 1405 \\
\hline 351.255 & 1066 & 421.328 & 8325 & 503.469 & 531 & 576.087 & 420 \\
\hline 352.257 & 969 & 422.332 & 2474 & 505.421 & 4848 & 576.502 & 3219 \\
\hline 353.267 & 10885 & 423.344 & 18638 & 506.426 & 1814 & 577.510 & 7516 \\
\hline 353.401 & 449 & 423.504 & 730 & 507.435 & 9461 & 578.514 & 3312 \\
\hline 354.274 & 12078 & 424.346 & 6066 & 508.438 & 3733 & 579.525 & 3810 \\
\hline 355.068 & 753 & 425.349 & 1345 & 509.446 & 1761 & 580.531 & 1392 \\
\hline 355.281 & 8953 & 435.034 & 788 & 513.079 & 1093 & 581.538 & 528 \\
\hline 356.285 & 1898 & 435.344 & 4484 & 514.084 & 549 & 590.510 & 470 \\
\hline 362.022 & 758 & 436.348 & 1271 & 515.085 & 563 & 591.130 & 487 \\
\hline 363.029 & 514 & 437.043 & 536 & 516.097 & 1364 & 591.528 & 539 \\
\hline 365.266 & 12028 & 437.359 & 7389 & 517.108 & 880 & 592.501 & 1265 \\
\hline 366.270 & 3408 & 438.364 & 2743 & 518.101 & 398 & 593.512 & 630 \\
\hline 367.282 & 71757 & 439.059 & 6591 & 519.434 & 2469 & 594.143 & 479 \\
\hline 367.975 & 593 & 439.365 & 942 & 520.439 & 1383 & 595.485 & 566 \\
\hline 368.104 & 649 & 440.062 & 2827 & 521.450 & 7273 & 605.503 & 524 \\
\hline 368.286 & 18589 & 441.058 & 1438 & 522.456 & 4063 & 606.519 & 526 \\
\hline 368.550 & 605 & 442.064 & 379 & 523.466 & 25762 & 786.696 & 370 \\
\hline
\end{tabular}

